\title{
A New Fuzzy Based Stability Index Using Predictive Vehicle Modeling and GPS Data
}

\author{
Benjamin Lawrence Blake Duprey
}

A thesis submitted to the Graduate Faculty of the Virginia Polytechnic Institute and State University in partial fulfillment of the requirements for the degree of

\author{
Master of Science \\ in \\ Mechanical Engineering \\ Saied Taheri, Ph.D, Chair \\ Mehdi Ahmadian, Ph.D \\ R. Gordon Kirk, Ph.D
}

May 4, 2009

Blacksburg, Virginia

Key Words: GPS, three degree-of-freedom, (3DOF), linear single track model, vehicle simulation, TruckSim, hardware-in-the-loop (HIL) simulator, fuzzy logic

Copyright 2009, Benjamin Lawrence Blake Duprey 


\title{
A New Fuzzy Based Stability Index Using Predictive Vehicle Modeling and GPS Data
}

\author{
Benjamin Lawrence Blake Duprey
}

\begin{abstract}
(ABSTRACT)
The use of global positioning systems, or GPS, as a means of logistical organization for fleet vehicles has become more widespread in recent years. The system has the ability to track vehicle location, report on diagnostic trouble codes, and keep tabs on maintenance schedules. This helps to improve the safety and productivity of the vehicles and their operators. Additionally, the increasing use of yaw and roll stability control in commercial trucks has contributed to an increased level of safety for truck drivers. However, these systems require the vehicle to begin a yaw or roll event before they assist in maintaining control. This thesis presents a new method for utilizing the GPS signal in conjunction with a new fuzzy logic-based stability index, the Total Safety Margin (TSM), to create a superior active safety system.
\end{abstract}

This thesis consists of four main components:

(1) An overview of GPS technology is presented with coverage of several automotive-based applications. The proposed implementation of GPS in the new Hardware-in-the-Loop (HIL) driving simulator under development at the Virginia Tech Center for Vehicle Systems and Safety (CVeSS) is presented.

(2) The three degree-of-freedom (3DOF), linear, single track equation set used in the Matlab simulations is derived from first principles.

(3) Matlab and TruckSim 7® simulations are performed for five vehicle masses and three forward velocities in a ramp-steer maneuver. Using fuzzy logic to develop the control rules for the Total Safety Margin (TSM), TSM matrices are built for both the Matlab and TruckSim 7 ${ }^{\circledR}$ results based on these testing conditions. By comparing these TSM matrices it is shown that the two simulation methods yield similar results.

(4) A discussion of the development and implementation of the aforementioned HIL driving simulator is presented, specifically the steering subsystem. Using Matlab/Simulink, dSPACE ControlDesk, and CarSim RT® software it is shown that the steering module is capable of steering the CarSim RT ${ }^{\circledR}$ simulation vehicle accurately within the physical range of the steering sensor used. 


\section{Contents}

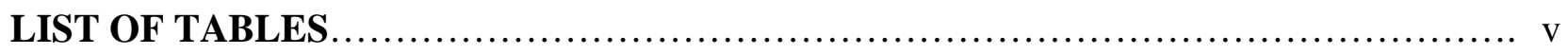

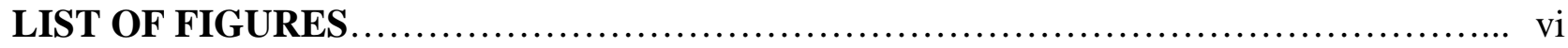

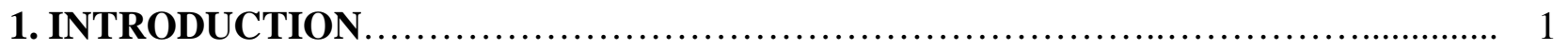

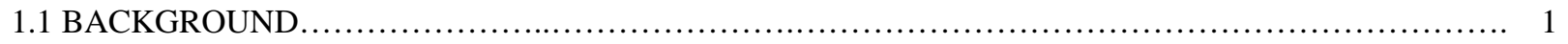

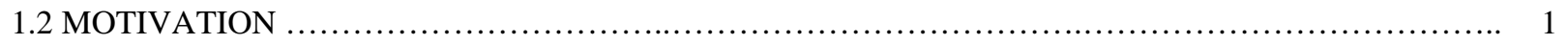

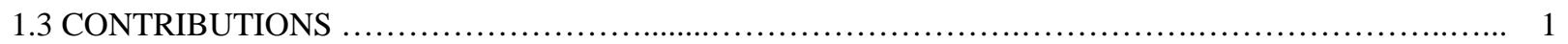

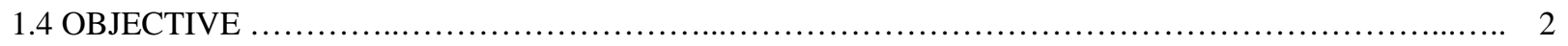

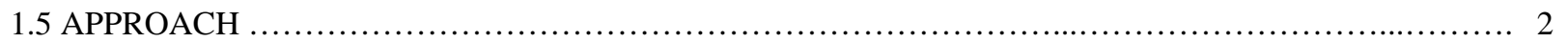

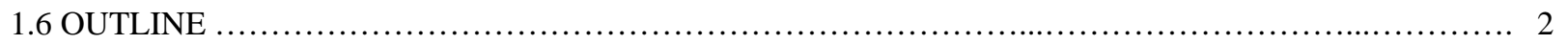

2. LITERATURE REVIEW ....................................................... 3

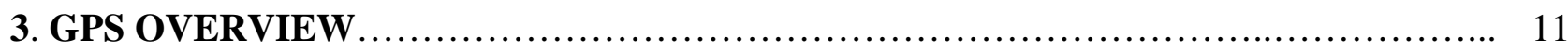

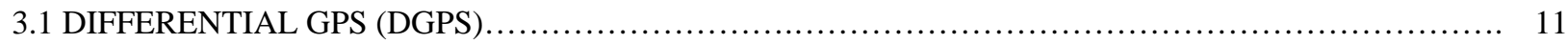

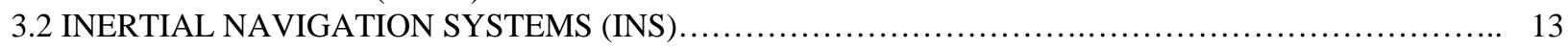

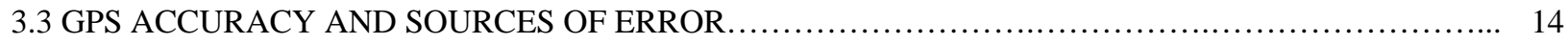

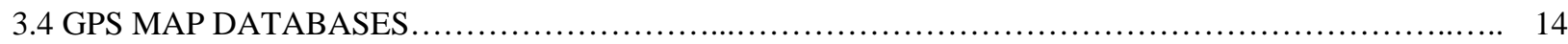

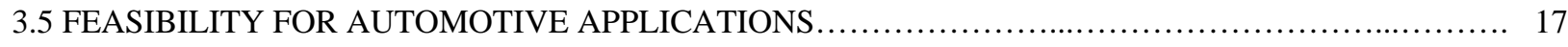

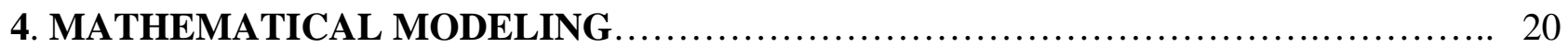

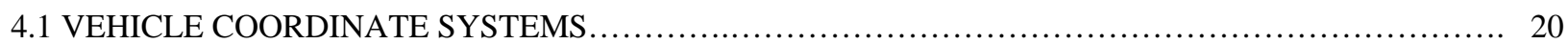

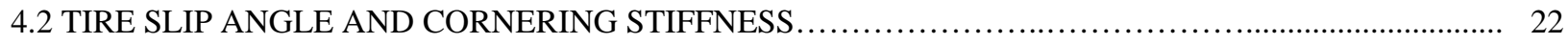

4.3 THREE DEGREE OF FREEDOM (DOF) VEHICLE DYNAMICS EQUATION ........................ 28

4.4 ROTATION MATIRCES, ANGULAR VELOCITIES, AND THE FINAL EQUATION ..................... 45

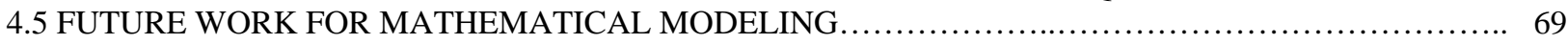

5. VEHICLE SIMULATIONS USING MATLAB AND TRUCKSIM 7 ................ 70

5.1 SIMULATIONS USING THE MATLAB 3 DOF MODEL AND TRUCKSIM 7 ...................... 70

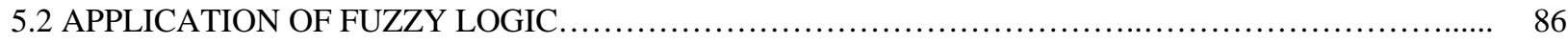

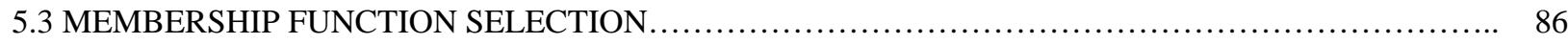

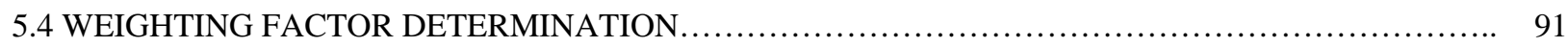

5.5 FUZZY LOGIC-BASED TOTAL SAFETY MARGIN (TSM) WITH GPS INPUT ..................... 92

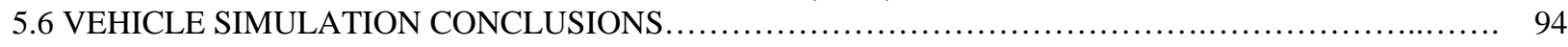

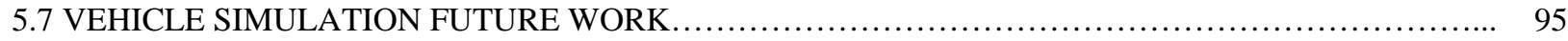

\section{HARDWARE-IN-THE-LOOP (HIL) SIMULATION: INTRODUCTION AND}

TECHNICAL OVERVIEW .................................................. 97

6.1 HIL SIMULATOR DESIGN: MAIN PLATFORM.................................................... 98

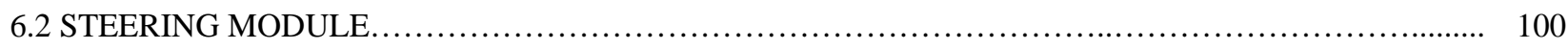

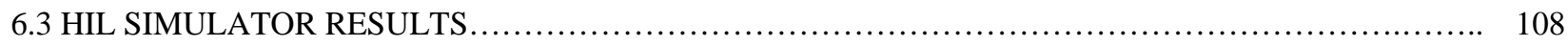

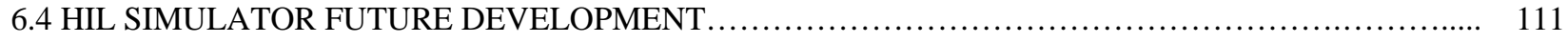

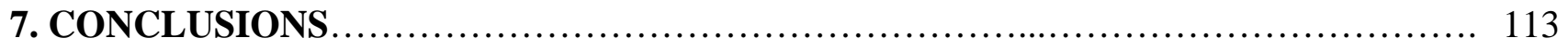

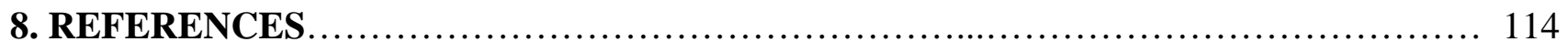

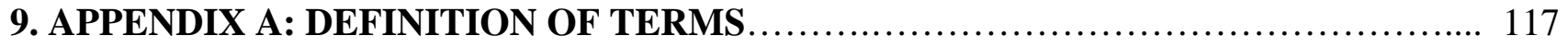


10. APPENDIX B: VEHICLE PARAMETERS..................................... 122

11. APPENDIX C: FUZZY LOGIC: A TECHNICAL OVERVIEW ..................... 123

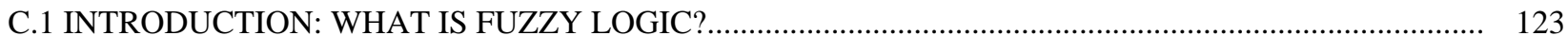

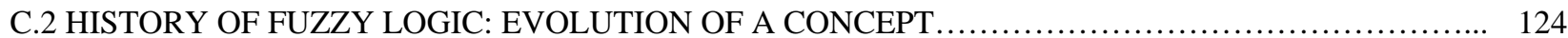

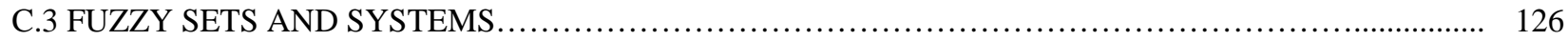




\section{List of Tables}

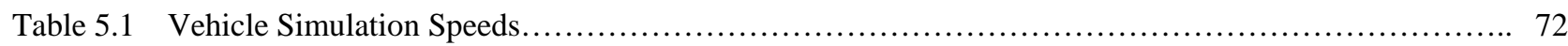

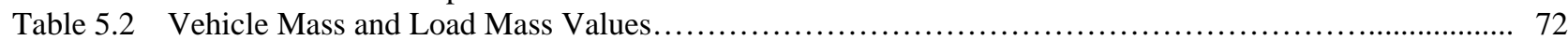

Table 5.3 Inertias of Baseline Vehicle with respect to vehicle sprung mass CG............................ 73

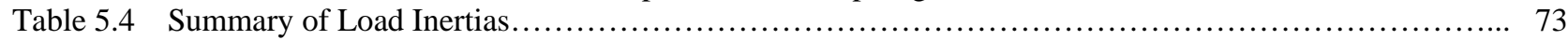

Table 5.5 Summary of Inertias of Load with respect to vehicle sprung mass CG............................... 74

Table 5.6 Summary of Total Vehicle Roll and Yaw Inertias with respect to vehicle sprung mass CG................. 74

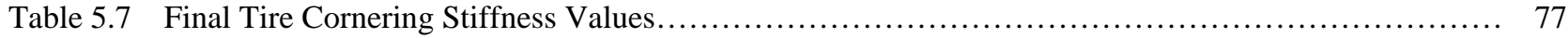

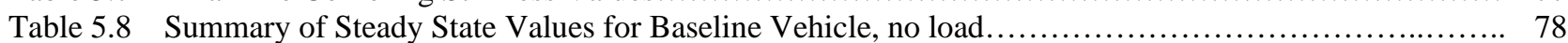

Table 5.9 Summary of Matlab and TruckSim Multimass Studies.......................................... 81

Table 5.10 Percent Error and Difference for Multimass Cases, Steady State Values.............................. 81

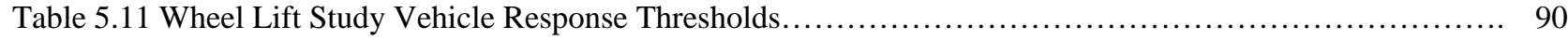

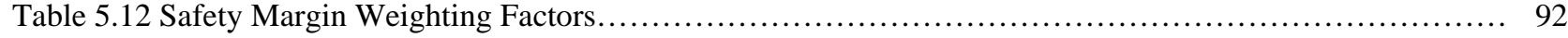

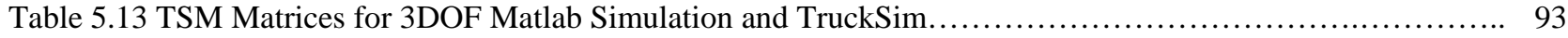

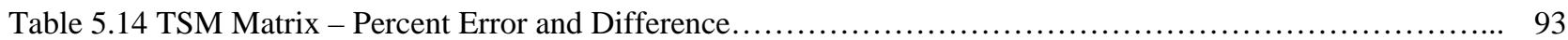




\section{List of Figures}

Figure $4.1 \quad$ SAE Vehicle Coordinate System.......................................................... 21

Figure 4.2 Simple vehicle model for roll in a right turn...................................................... 21

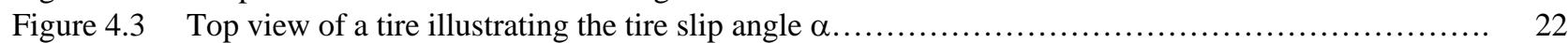

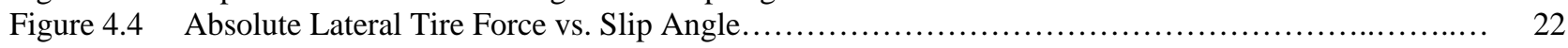

Figure 4.5 Absolute Lateral Tire Force vs. Slip Angle, showing linear tire properties........................ 23

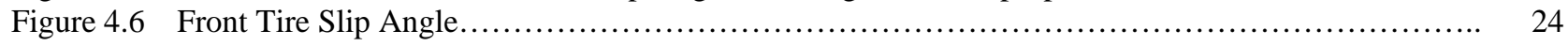

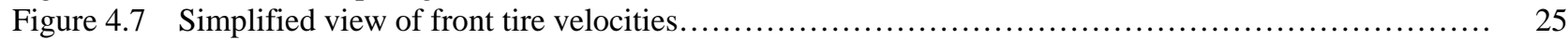

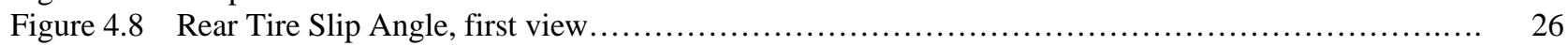

Figure 4.9 Rear Tire Slip Angle, second view......................................................... 27

Figure 4.10 Top view of generic vehicle driving straight but with a positive, nonzero heading angle $\psi$ with respect to the Earth-fixed coordinate system in the X-Y plane....................................... 29

Figure 4.11 Simple car model with side force characteristics for front and rear (driven) axles, yaw plane........... 41

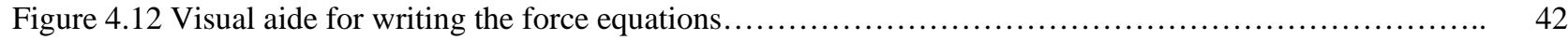

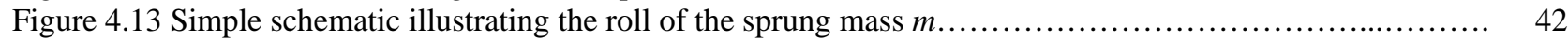

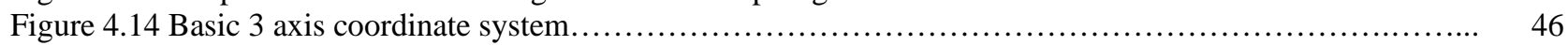

Figure 4.15 Illustrating the lateral translational velocity term of the kinetic energy.............................. 51

Figure 4.16 Simplified top view of vehicle illustrating longitudinal translational velocity term of kinetic energy... 52

Figure 5.1 Vehicle executing a constant radius turn from straight ahead driving.............................. 71

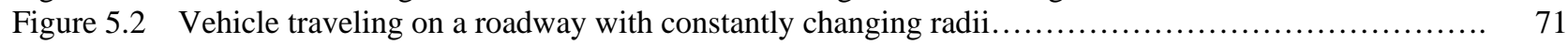

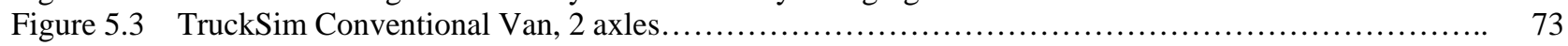

Figure 5.4 TruckSim Tire Lateral Force vs. Slip Angle for $3000 \mathrm{~kg}$ Load Rated Tire.............................. 75

Figure 5.5a TruckSim Open Loop Steering Controller................................................... 76

Figure 5.5b TruckSim Steering Controller and Matlab 3DOF smoothed step................................ 76

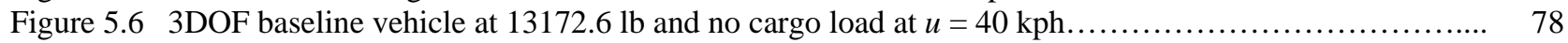

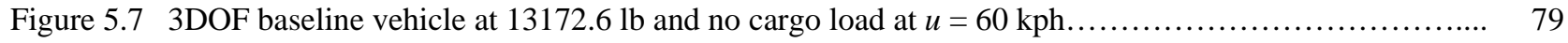

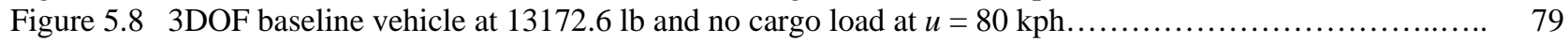

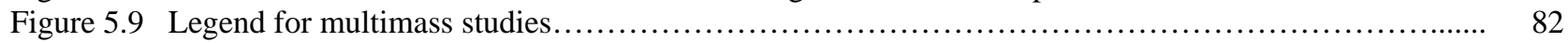

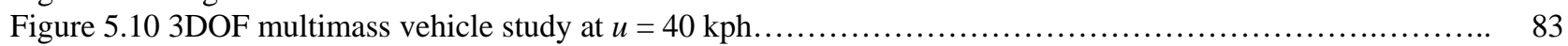

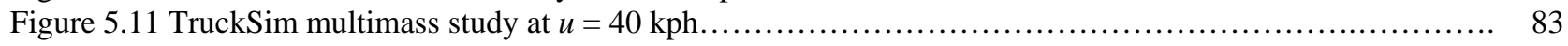

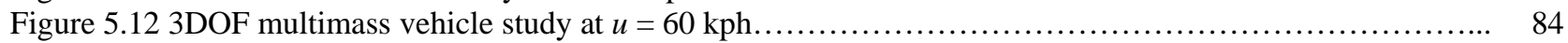

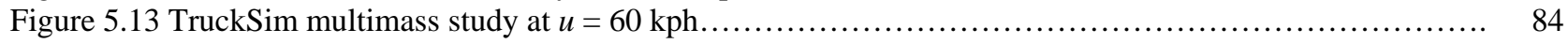

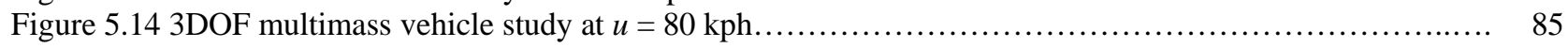

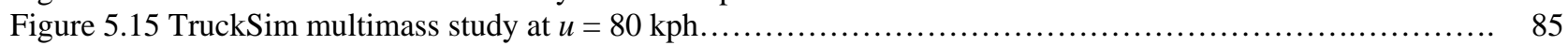

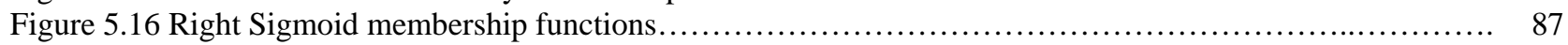

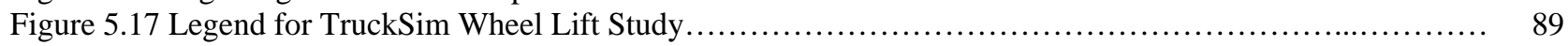

Figure 5.18 Speed Comparison Chart for TruckSim 7 Wheel Lift Speeds................................... 89

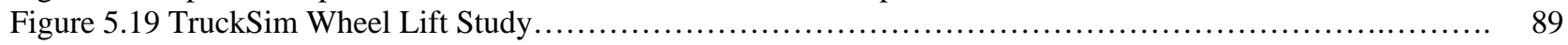

Figure 5.20 Lateral Acceleration vs. Radius of Curvature assuming uniform circular motion..................... 94

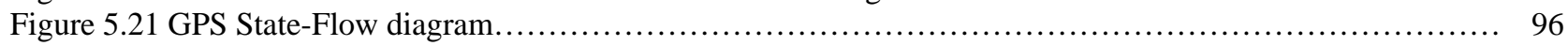

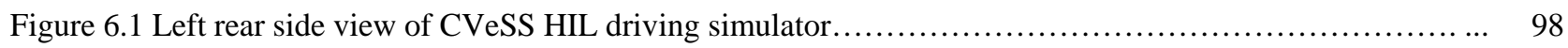

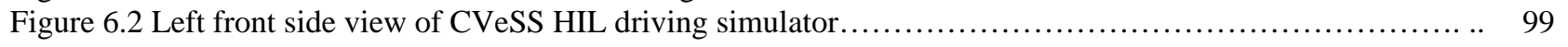

Figure 6.3 Left side view of CVeSS HIL driving simulator........................................... 99

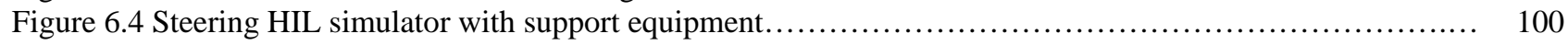

Figure 6.5 dSPACE MicroAutoBox 1401/1501 and power supply ......................................... 100

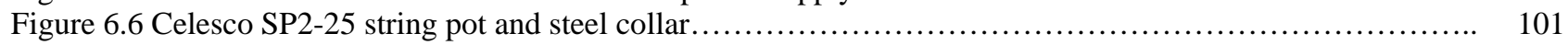

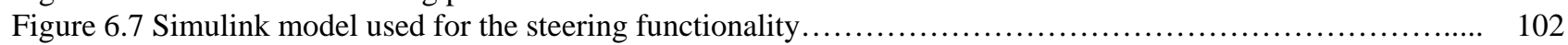

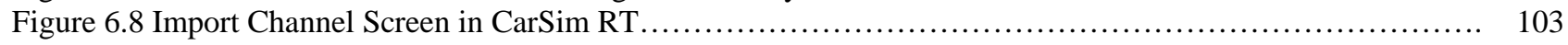

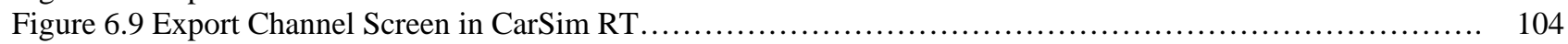

Figure 6.10 Source Block Parameters for Analog / Digital Converter block in Simulink.......................... 105

Figure 6.11 String pot signal output as read by dSPACE vs. Steering Shaft Rotation............................ 106

Figure 6.12 Build Model option in the Tools, Real Time Workshop menu................................... 107 


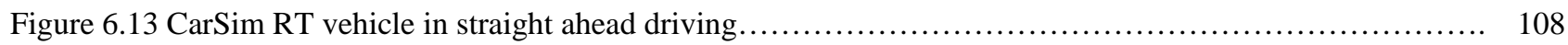

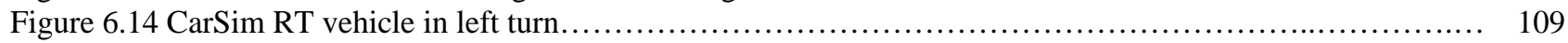

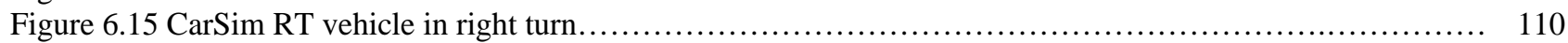

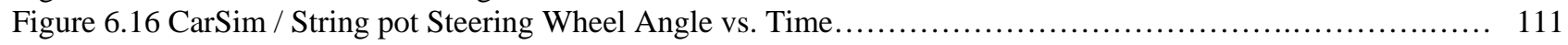

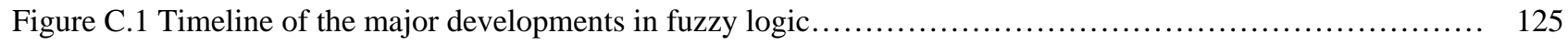




\section{Chapter 1}

\section{Introduction}

\subsection{Background}

Due to the variability in vehicle design, loading, and overall vehicle configurations, certain vehicle combinations exhibit oversteer throughout their operating range. A vehicle that is oversteer can become yaw divergent at certain levels of lateral acceleration, while a vehicle that is understeer cannot. Depending on the vehicle setup, yaw instability can occur at significantly lower lateral accelerations than rollover. Currently available stability control systems require the vehicle to begin an oversteer, understeer, or roll event before any brake control system interaction can take place.

\subsection{Motivation}

From a safety standpoint, what if it were possible to predict the vehicle behavior for the upcoming road geometry based on the current vehicle operating conditions, where that information could be used to slow the vehicle down, or to provide preemptive brake controls to reduce or eliminate the risk of an understeer, oversteer, or roll event? Such is the goal of this thesis, in the form of the fuzzy logic-based stability index, the Total Safety Margin (TSM).

\subsection{Contributions}

At least two publications [3,6] have used GPS as a prediction tool for vehicle dynamics, and in both instances the GPS provided either matching or improved data over the vehicle mounted sensors used for ground reference. Additionally, there have been many notable research publications describing the efforts to incorporate GPS map tracking and fuzzy logic-based lateral control. For example, the work done by Jose Naranjo, et al. [1] on lateral vehicle control involving fuzzy logic techniques centered around the use of GPS tracking as a means to control the vehicle steering, with autonomous vehicles being the ultimate goal. The work by Hessburg and Tomizuka [17] also used a fuzzy rule-based controller as a means for lateral control but there the intent was for use in an automated highway system. 


\subsection{Objective}

The objective is to develop a fuzzy logic-based stability index, called the Total Safety Margin (TSM), to predict the overall vehicle stability condition. The GPS signal would provide data on the upcoming road geometry.

\subsection{Approach}

This system works by taking the GPS signal and providing the road geometry data to the predictive vehicle dynamics model. This model finds the vehicle's lateral velocity, yaw rate, roll angle, and roll angle rate. The lateral acceleration is then calculated. These vehicle responses are mathematically determined based on the maneuver being performed, such as a ramp steering input to a constant radius turn. The maximum values of the lateral acceleration, lateral velocity, yaw rate, and roll angle are then found. Using a right sigmoid membership function in the fuzzy logic process, these maximum values are used to determine the safety margins for lateral acceleration, lateral velocity, yaw rate, and roll angle. These safety margins are combined with system weighting factors to collectively determine the Total Safety Margin (TSM).

\subsection{Outline}

This process begins with a technical overview of GPS technology followed by a brief survey of current automotive applications. The proposed application for GPS as it pertains to the fuzzy logic-based stability index, as well as implementation on the hardware-in-the-loop (HIL) driving simulator, is discussed in Chapter 3. A detailed derivation of the three degree-of-freedom, linear, single track model used in the Matlab simulations make up Chapter 4. Since real vehicle data was not available for validation purposes, TruckSim $7 \AA$ was used for comparison with the Matlab model. The results of this comparison, as well as the development of the fuzzy logicbased stability index, are presented in Chapter 5. In parallel development, a hardware-in-the-loop (HIL) driving simulator has been under construction at the Virginia Tech Center for Vehicle Systems and Safety (CVeSS). The intention of this simulator is to allow users to use a 3-D vehicle dynamics simulation program such as TruckSim $7 \circledast$ to test brake and stability control algorithms prior to in-vehicle testing. The first module of this new simulator, the steering module, is now fully functional. The development and preliminary testing of this simulator utilizing CarSim RT® is covered in Chapter 6.

Definitions of important terms are listed in Appendix A. 


\section{Chapter 2}

\section{Literature Review}

There have been many notable research publications describing the efforts to apply various fuzzy logic membership functions to both automotive and non automotive fuzzy logic-based predictive methodologies. For example, the work by Jose Naranjo, et al. [1] on lateral vehicle control involving fuzzy logic techniques centered on the use of GPS tracking as a means to control the vehicle steering. Their main sensorial input was a real-time kinematic differential GPS (RTK DGPS) which provided positioning precision of one centimeter. That study utilized modified trapezoidal membership functions, one each for angular error and lateral error. Their ultimate goal was autonomous vehicles, and the experimental results showed that with the RTK DGPS providing positioning with one centimeter precision, it was possible to maintain a vehicle in its lane on a closed road course.

Like Naranjo, et al. [1], the work of Hessburg and Tomizuka [17] also used a fuzzy rule-based controller as a means for lateral control, but that system was intended was for use in an automated highway system. The fuzzy rules were developed based on human driver's experiences. They were designed to track the center of a lane in the presence of external disturbances and over a range of vehicle operating conditions. In the case of severe road curvature, special rules were developed to use feed forward steering action, utilizing preview information regarding the characteristics of the upcoming curve. The grade of membership (i.e. fuzzification) of the input variables was calculated using triangular membership functions. The weighed average defuzzification method was used.

Boada, Boada, and Diaz [18] discussed a fuzzy logic-based yaw moment controller to improve vehicle handling and stability. The controller generated the necessary brake torque to control the yaw moment for the steering inputs tested. The controller was also able to adapt to different driving conditions including dynamic maneuvers, varying initial speeds, and road surfaces. Their controller used triangular membership functions with five fuzzy sets for the input and seven for the output. Defuzzification was via the center of area method.

An example of a non automotive-based fuzzy logic application is discussed in [19]. In this case, an Adaptive Neuro-Fuzzy Inference System (ANFIS) was used to investigate the stock price trend of the Iran Khodro Company at the Tehran Stock Exchange. A Takagi-Sugeno model was used for designing the pattern for ANFIS. Two inputs were assumed for the desired fuzzy inference system, and the ultimate output was calculated using the weighted average 
defuzzification method. A variety of membership functions including triangular, trapezoidal, and Gaussian combination were used and it was concluded that ANFIS was capable of forecasting Iran Khodro's stock price behavior.

Khanafer, et al. [35] discussed a rule based collision avoidance system which focused on scene interpretation of a situation and its optimization. A field test study was set up to take the driver's behavior into account. Using different strategies, optimization of all parameters, including the parameters of the membership functions, weights, and conclusions, was achieved. This process led to the conclusion that the driver assesses the threat of a situation later than the designed system, prior to optimization. Once optimization had been achieved, the two fuzzy systems, one each for lateral and longitudinal directions, were connected by another fuzzy system to decide how to react. The required steering maneuver for accident avoidance was described by a sigmoidal function.

A search of the literature revealed two applications of driver notification due to potentially unstable turning and braking events, with steering, braking, and suspension intervention if necessary. In the case of Davoudi, et al. [36], a fuzzy based vehicle dynamic stability control (FDSC) was investigated and simulated in the Matlab/Simulink environment. The paper introduced a calculation for the vehicle's center of gravity (CG) movements. The main objective was to analyze the behavior of the CG within the vehicle perimeter, as defined by the height of the main suspension components, specifically the springs and dampers. The fuzzy controller used sensors and actuators to prevent rollover in critical situations. These included actuators to change the suspension height, as well as brake-by and steer-by-wire systems. Triangular membership functions were used for the position and velocity of the vehicle CG. The center of area (centroid) method was used for defuzzification, and the output of the fuzzy analyzer was a signal that represented a critical situation. The actuators in this fuzzy based vehicle dynamic control system monitored this signal and acted when needed. An alarm system notified the driver of instability before it occurred.

In [23], Ehlbeck, et al. discussed one method for reducing heavy truck rollover accidents, specifically the Freightliner/MeritorWABCO Roll Advisory and Control System (RA\&C). The system consisted of four main functions:

(1) Roll Risk Detection

(2) Roll Stability Advisor (RSA)

(3) Hard Braking Event Detector (HBED)

(4) Roll Stability Control (RSC)

The paper covered rollover dynamics basics, determination of critical lateral acceleration, and rollover prevention methods. The block diagrams for the RA\&C system, RSC control algorithm, and RSA were also presented and discussed. 
The RSA and HBED systems were designed to reduce accidents by training drivers to recognize and avoid dangerous driving conditions. These systems were feedback in nature, meaning they operated by sensing when lateral acceleration or braking "risk" conditions occurred. The information was displayed to the driver immediately at the end of the event. The intent was to train the driver to avoid these situations in the future with such messages as "High Risk of Rollover Detected" and "Reduce Speed at Least $10 \mathrm{mph}$ ”. The goal of the system was to present timely performance feedback to help the driver reduce the incidence of rollover risk and hard braking events.

The goal of the RSC system was to eliminate rollovers. It functioned to identify critical rollover situations and endeavored to assist the driver in safely completing the maneuver. This was accomplished by temporarily intervening to rapidly reduce the vehicle's speed via engine torque reduction.

Regarding rollover analysis, the paper by Bernard, Shannan, and Vanderploeg [37] presented various mathematical models of vehicle rollover, ranging from simple quasi-static models which required knowledge of only a few basic parameters, to simulations with increasing complexity and increasing need of measurement data. Included was a discussion of the tilt table test, a simplified metric to estimate the maximum cornering limit of a vehicle. This test represented the maximum sustainable lateral acceleration of a vehicle in a steady state turn. The paper also discussed the results of computer simulations for an automobile, a utility vehicle, and a truck. By comparing three vehicle types, a range of vehicle dynamics responses were reported. This approach was instructive since this thesis endeavored to determine a range of vehicle dynamics responses too, albeit for a single vehicle type but at multiple loading conditions. The computer simulations described in [37] encompassed several maneuvers including a J-turn and step-steer input. Comparing these results with those obtained from the Matlab and TruckSim 7 simulation cases presented in this thesis, it will be shown that the Matlab and TruckSim 7 results fall within the predicted range set by the utility vehicle and truck.

The goal of the Riede, Leffert, and Cobb [15] paper was to describe the effects various vehicle parameters had on vehicle dynamics characteristics. For example, for the inertia estimation cases, it was shown that the vertical and longitudinal sprung and unsprung mass locations were close to one another. This estimation was a useful starting point when considering the effects of the loading conditions used in the multimass study performed in this thesis.

Isik [16] reviews the basic principles of fuzzy logic and summarizes in bullet point-form significant applications such as automatic transmission controllers from Nissan, Mazda, and Honda; heat pump and air conditioning controls from Mitsubishi; and construction project optimization for the Konoike Construction company. The paper clearly outlines the feasibility of fuzzy logic as compared to classical logic:

(1) Fuzzy logic allows truth values which are fuzzy sets with labels such as true, somewhat true, and not quite true.

(2) Fuzzy logic allows a range of quantifiers such as usually, quite a few, and many. 
(3) Labels such as likely and highly unlikely can be modeled as fuzzy probabilities and used for qualitative decision making.

(4) Fuzzy logic provides mechanisms for dealing with hedges such as very, more or less, and not quite.

Adaptive fuzzy systems are also covered, using an example of driving in snow vs. dry conditions. Since the road conditions affect acceleration and deceleration, transmission shifting and vehicle turning to name a few, Isik points out the advantages of modifying the sensory data rather than the fuzzy rule base for fuzzy logic-based controllers. This information proved useful when designing the fuzzy rules for this thesis.

Like Isik [16], Kosko [20], Klir [21], and Piegat [22] discussed the theory and application of fuzzy logic in text book form. Coverage included:

(1) Fuzzy logic usage in automotive and nonautomotive applications such as automatic transmissions, robotic controllers, subways, medical science applications including blood pressure controls, and word recognition.

(2) Fuzzy rules and their structure, specifically the linguistic nature of fuzzy system definitions.

(3) Membership function types including triangular, trapezoidal, Gaussian, and left and right sigmoids. The advantages and disadvantages of each type are explained so as to provide the interested party enough information to make a proper selection.

(4) Defuzzification techniques such as the standard additive model, center of area, center of gravity, center of maxima, and the weighted average. This proved to be useful since the weighted average method, which includes the system weighting factors, requires less information about the fuzzy system being modeled than other methods, and still provides a meaningful representation using fuzzy logic application. This was most useful since the stability index presented in this thesis, the Total Safety Margin (TSM), has the form of a weighted average and is still in the early development phase.

(5) Use of weighting factors in the defuzzification process to scale each term to reflect rule credibility or in the case of this thesis the importance of each dynamic response specific to the vehicle type being modeled.

Different methods for obtaining weighting factors have been described in the literature. For example, a decision tool was developed at Teledyne Ryan Aeronautical [38] which was designed to produce a decision under conditions in which the decisions are difficult to produce and substantiate. In this instance, weighting factors were computed from the available system facts and used to determine the best candidate by scaling available system parameters. This was conceptually critical to this thesis since the updated Total Safety Margin (TSM) used weighting factors that were calculated based on vehicle response data and are no longer assigned a priori, as they were in [24]. 
Kaplan, et al. [2], Logsdon [4], and Hoffmann-Wellenhoff, et al. [5] discussed GPS theory and concepts. Coverage included:

(1) Math models, parameters, and figures detailing Keplerian and Perturbed motion, as well as the math models and geometry for atmospheric effects such as ionospheric and troposheric path delays.

(2) Sensor integration in land vehicle systems, including inertial navigation systems (INS), emergency messaging, and vehicle heading change determination.

(3) Pseudorange and differential GPS (DGPS), both of which are key concepts in the use and implementation of GPS to ground vehicle navigation.

Since one of the novel features of the Total Safety Margin (TSM) presented in this thesis is the incorporation of the GPS signal to provide the road geometry data to the predictive vehicle dynamics methodology, these resources provided the necessary GPS background material.

Venhovens, et al. [3] of BMW described how GPS was used to supplement several vehicle systems: Situation Adaptive Drivetrain Management (SAM), Adaptive Light Control (ALC), and Autonomous Speed Adaptation. The digitized road map and GPS navigation system formed the basis for the Situation Adaptive Drivetrain Management (SAM). Their main objective was to increase fuel efficiency and cut exhaust emissions by adapting the throttle, brake, and gears to the current traffic situation. The driving situation several seconds ahead could be anticipated and if needed, possible interventions of the drivetrain made using the GPS and additional vehicle data.

The Adaptive Light Control (ALC) system controlled the headlamps using the vehicle trajectory prediction based on vehicle dynamics and/or route vectors from the navigation system. The BMW team found that in their tests with real vehicles and the standard GPS accuracy under 2dimensional situations, the vehicle dynamics-based path prediction and the 2-dimensional navigation data used to control the headlamps was limited to certain road geometry. It was insufficient when encountering hilly roads, s-curves and other types of special road constructions. This was because the accuracy of the standard GPS and map databases was insufficient. Additional knowledge was necessary to overcome those deficiencies which led to the simulation and use of highly accurate differential GPS (DGPS) and road databases. For this ALC system, the driving dynamic status of the car, coupled with the three-dimensional DGPS data via a Kalman filter and bi-splines, was used to derive the shape of the road.

In the case of the Autonomous Speed Adaptation, the maximum acceptable speed in turns was estimated based on the road geometry and the vehicle's dynamics. According to BMW, the three main parameters for curve speed estimation are the curve radius, the change of direction of a turn and the roadway type.

Perhaps most important for the feasibility standpoint of this paper, the estimated safe speed is compared with the current vehicle speed. If the speed of travel is too high, either a warning is 
issued or automatic deceleration will take place. This concept matches the design intent of the GPS-assisted vehicle predictive system presented in this thesis.

Beiker, et al. [6] of BMW clearly explains that GPS assistance must only be used to enhance but not to ensure functionality of safety critical systems. Dynamic testing of a real vehicle was performed on surfaces with different friction levels. Using a standard vehicle dynamics data collection tool, an optical $5^{\text {th }}$ wheel, test results were compared to those obtained using a GPS / INS unit and it was shown that the GPS / INS and $5^{\text {th }}$ wheel data matched very closely. In doing so, the paper was able to show that proof of feasibility for an automotive GPS/INS setup needs to cover the following steps to check both performance and practicability:

(1) The performance of the basic concept needs to be compared to a reference system to prove accuracy and reliability.

(2) The proposed GPS/INS setup needs to be tested in various maneuvers and under various conditions to prove the practicability of such a system, as well as its benefits for automotive applications.

With respect to this thesis, these two papers proved that GPS has been successfully implemented in vehicle dynamics prediction applications.

Dixon [7], Gillespie [8], Jazar [9], Karnopp [10], Milliken and Milliken [11], Pacejka [12], and Wong [13] provided the necessary coverage of basic vehicle dynamics topics. These included:

(1) Coverage of the SAE vehicle coordinate system.

(2) Coverage of the pneumatic tire coordinate system, as well as the conceptual and mathematical meaning of the linear region of such a tire.

(3) Steady-State handling, including understeer and oversteer. In this instance steady-state handling is taken to mean the absence of ground or wind disturbances as well as fixed controls, i.e. constant path radius of curvature.

(4) Transient handling, in the form of a step steering input.

(5) Coverage of the common two degree-of-freedom (2DOF) single track, or bicycle, model.

(6) Varying levels of coverage of the less common three degree-of-freedom (3DOF), single track model, specifically with the third degree of freedom in the roll plane.

A common disadvantage of moderately complex mathematical models such as the 3DOF single track model is the lack of sufficient detail in commonly published material. Pacejka [12] provided some detail, but major steps are missing, including the necessary but non-intuitively obvious Euler Angle coordinate transformations which are used in the derivations of the kinetic and potential energy equations. These are necessary to derive the angular velocities of the body, which in turn are used in the Lagrangian method of derivation presented in this thesis. Spacecraft 
dynamics books are well suited to this task due to the fact that Euler Angle transformations are common in the analysis and control of satellite dynamics. To this end, Sidi [14] provided a clear explanation of common Euler Angle definitions and transformation matrices. The two types of Euler Angle rotations are:

(1) Successive rotations about each of the three axes in a 3-D coordinate system.

(2) First and third rotations about the same axis, with the second rotation about one of the two remaining axes.

A Hardware-in-the-Loop (HIL) simulator is a laboratory test environment which combines computer simulation with embedded hardware. Common automotive setups can be used to investigate vehicle steering, braking, handling, or testing of an automotive ECU (electronic control unit). For example, Velardocchia and Sorniotti [25] documented the necessary tests to characterize, on the bench, a commercially available electronic stability control (ESC) hydraulic control unit (HCU) using the Politecnico di Torino Hardware-in-the-Loop brake systems test bench. Their tests included studying the step responses of each valve as well as the pump motor. Using this HIL simulator they go on to compare different commercial ABS strategies in a large spectrum of maneuvers, including slow and panic brake applications as well as split- $\mu$ and alternating- $\mu$ maneuvers [26]. Kohl and Jegminat of dSPACE GmbH described the various test phases during the development of automotive electronics using HIL simulation [27], while Schuette and Waeltermann, also of dSPACE GmbH, provided a technical survey of state-of-theart HIL test systems for vehicle dynamics controllers. These controllers included ABS, TCS, and ESC functionality [28]. Segawa, Nakano, Shino, and Nagai conducted a preliminary study concerning the quantitative analysis of a passenger vehicle steering system using HIL simulation. The purpose of their study was to develop a steering HIL simulator that could quantitatively evaluate different steering systems. Critical to this evaluation was the force feedback to the steering wheel which was accomplished via a controllable force on the tie rod [29]. In [30] Kim, Lee, and Yu discussed the development of a vehicle simulator using a GSS6560 real-time GPS simulator from Spirent Corp. Their system consisted of a full motion HIL driving simulator with a 360 degree viewing screen utilizing a 3D virtual space to reflect a part of a city in a real world environment. Their goal was telematics software development. The methodology and implementation outlined in [30] should prove useful once the GPS component of the driving simulator being constructed at CVeSS reaches a further stage of maturity.

Since the HIL simulator presented in this thesis was developed from scratch, these HIL simulation papers provided useful information on the capabilities and limitations of current automotive-based HIL systems.

The goal for this thesis is to update the Total Safety Margin (TSM), a fuzzy logic-based stability index first presented in [24] using the weighted average defuzzification method. As defined in that paper, the fuzzy logic-based Total Safety Margin (TSM) was an attempt to define the stability level of a vehicle as a single, non-dimensional number. The TSM presented in that paper was also based on a single vehicle speed and utilized a smooth step for both the steering input and the fuzzy logic membership functions. Furthermore, the weighting factors used in that study were determined arbitrarily based on the vehicle type being considered. The weighting factors 
are now calculated based on vehicle response data and are no longer assigned a priori, as in [24]. The weighting factor calculation is based on a comparison between results from a ramp-steer maneuver run at $80 \mathrm{kph}$ and a lifted wheel study. Furthermore, the steering input has also been updated. It is still a ramp steer input to the same maximum steering wheel angle as in [24], but it has been slowed down such that it now occurs over a period of one second. Two additional longitudinal vehicle velocities have been added to the study. This was done to provide a more complete coverage of the working vehicle speed range as well as to gain a better understanding of the vehicle dynamics behavior. Additionally, this thesis presents the use of new right sigmoid membership functions to calculate the membership grades of the individual safety margins used in the Total Safety Margin (TSM). Combined with the GPS signal input in the form of road radius data, this thesis presents a novel technique for predicting the vehicle dynamics behavior. 


\section{Chapter 3}

\section{GPS Overview}

The development of the Global Positioning System (GPS) was initiated in the early 1960s by several U.S. Government organizations including the Department of Defense (DOD), NASA and the Department of Transportation (DOT) for three-dimensional position determination. In 1969 the Office of the Secretary of Defense (OSD) established the Defense Navigation Satellite System (DNSS) program and from this effort, the system concept for NAVSTAR GPS was formed [4]. Presently, GPS is fully operational and meets the criteria established in the 1960s for an optimum positioning system. The system provides accurate, continuous, world-wide, threedimensional position and velocity information to users with the appropriate receiving equipment [2-3]. The satellites are launched into 10,898 nautical mile orbits in 6 orbital planes, each tipped 55 degrees with respect to the equator. The complete constellation consists of 21 NAVSTAR satellites plus 3 active on-orbit spares [4]. GPS utilizes the concept of one-way time-of-arrival (TOA) ranging enabling the receiver to determine the satellite-to-user range. At least four satellites are required to determine the user latitude, longitude, height, and receiver clock offset for internal system time [2-3]. This is accomplished by the simple resection process using the distances measured to satellites [5]. The NAVSTAR GPS uses pseudoranging techniques rather than Doppler shift measurements to fix the user's position. This means that a NAVSTAR receiver measures the signal travel times from several satellites simultaneously, instead of frequency variations from only one satellite at a time [4].

\subsection{Differential GPS (DGPS)}

Differential GPS (DGPS) is a method to improve the positioning or timing performance of GPS using one or more ground-based reference stations at known (i.e. fixed) locations, each equipped with at least one GPS receiver [2]. The stations broadcast the difference between the positions indicated by the satellite systems (i.e. the measured satellite pseudoranges) and their known fixed positions. Since a reference station calculates differential corrections for its specific location and time, users can incorporate these corrections to improve the accuracy of their position solution. Satellite ephemeris errors, as well as errors introduced via ionospheric and tropospheric distortions, can cause positioning errors to be spatially correlated, meaning that position solutions for users further away from the reference receiver will be less accurate than those closer to the reference station [3]. 
The reference stations provide information to the end user via a data link that may include:

- Corrections to the raw end user's pseudorange measurements, corrections to GPS satellite-provided clock and ephemeris data, or data to replace the broadcast clock and ephemeris information

- Raw reference station measurements (e.g., pseudorange and carrier phase)

- Integrity data (e.g., “use” or “don’t use” indications for each visible satellite, or statistical indicators of the accuracy of provided corrections)

DGPS techniques may be categorized in different ways: absolute or relative differential positioning; as local, regional, or wide area; and as code or carrier based [2].

Absolute differential positioning is the determination of the user's position with respect to an Earth-Centered Earth-Fixed (ECEF) coordinate system. This is the most common goal of DGPS systems. For absolute differential positioning, each reference station's position must be accurately known with respect to the same ECEF coordinate system in which the user position is desired. In contrast, relative differential positioning is the determination of the user's position with respect to a coordinate system attached to the reference stations, whose absolute ECEF positions may not be perfectly known [2].

DGPS systems may also be categorized in terms of the geographic area that is to be served. The simplest DGPS systems are designed to function only over a very small geographic area, with the user separated by less than 10-100 km from a single reference station. Multiple reference stations and different algorithms are typically involved to effectively cover larger geographic areas. The terms regional area and wide area are frequently used to describe DGPS systems covering larger geographic regions, with regional-area systems generally covering up to around a 1,000 km radius from the reference station, and wide-area systems covering larger regions (i.e. larger than $1,000 \mathrm{~km}$ radii) [2].

When wide-area augmentation is used in conjunction with code-based differential GPS, just a couple of reference receivers could cover an area as large as the United States. Its principle is based on estimating the errors of each component for the entire region, rather than only at the station positions [3].

One final categorization of DGPS systems is between so-called code-based and carrier-based techniques. Code-based DGPS systems rely primarily on GPS code, or pseudorange, measurements and can provide decimeter-level position accuracies. Carrier-based DGPS systems, on the other hand, rely primarily on carrier-phase measurements. They require more base stations than code-based differential techniques [3] but are much more precise than pseudorange measurements and can provide millimeter-level performance [2]. 


\subsection{Inertial Navigation Systems (INS)}

GPS receivers can be thought of as discrete-time position/velocity sensors with sampling intervals of approximately 1 second. The need to provide continuous navigation between the update periods of the GPS receiver, during periods of shading of the GPS receiver's antenna, and through periods of interference is the impetus for integrating GPS with various additional sensors. The most popular are inertial sensors typically measuring yaw rate and acceleration, but altimeters, speedometers, and odometers can be used. The most widely used method for this integration is the Kalman filter. One of the key attributes of the Kalman filter is that it provides a means of inferring information by the use of indirect measurements, the GPS measurements. It does not have to read control variables directly, but it can read an indirect measurement and estimate the control variables which, in GPS applications, are position, velocity, and possible attitude errors [2-3].

Employing GPS and inertial sensors for navigation is a complimentary relationship: the GPS sensor provides bounded accuracy and can calibrate the INS sensor when its own accuracy degrades over time, and the INS can support GPS receiver performance issues such as interference from external sources, time to first fix (i.e., first position solution), interruption of the satellite signal due to blockage by terrain or manmade structures such as buildings and tunnels, as well as signal integrity and reacquisition capability. Thus the integration of these two types of sensors not only overcomes performance issues found in each individual sensor, but also produces a system whose performance exceeds that of the individual sensors [2-3].

The discrete-time nature of the GPS solution is of concern in real-time applications such as vehicle control. When only three usable satellite signals are available, most receivers revert to a two-dimensional navigation mode by utilizing either the last known height or a height obtained from an external source. If the number of usable satellites is less than three, some receivers have the option of not producing a solution at all or extrapolating the last position and velocity forward, a process called dead-reckoning (DR) navigation. If a vehicle's path changes between GPS signal updates, this extrapolation of the last GPS measurement produces an error in the estimated and true position. The inertial navigation systems can therefore be used as a flywheel to provide navigation during shading outages [2-3].

When an INS is calibrated using a Kalman filter it can be used to improve GPS receiver performance in two additional ways. First, the information maintained by the integration filter can be used to reduce the time to reacquire GPS signals that have been lost through interference or obstruction; second, the integration filter can be used to aid the receiver's tracking loops, extending the thresholds for signal tracking [2].

The quality of INS very much depends on the quality of the inertial sensor used. With automotive-quality yaw rate sensors in combination with the wheel speed signals used by the ABS system, the position of a vehicle can be propagated up to $30 \mathrm{sec}$. with an error of less than $1 \mathrm{~m}$ [3]. The most significant factor related to the quality of an inertial system is the drift of a typical yaw rate sensor [3]. 


\subsection{GPS Accuracy and Sources of Error}

The standard GPS currently available for civil applications enables positioning with an accuracy of at least $33 \mathrm{~m}$, or a $1 \sigma$ horizontal, vertical, or three-dimensional position error as a function of satellite geometry and the $1 \sigma$ range error [2-3]. More accurate precision navigation can be obtained by Differential GPS based on error removal using the difference between a reference receiver's surveyed position and its precisely-known geographic location. As mentioned above, code-based differential techniques can provide decimeter-level position accuracy whereas carrier-based techniques, using the GPS satellite signal carrier frequency, can provide millimeter-level positioning.

Common sources for inaccuracies are:

- satellite clock error

- ephemeris prediction error

- atmospheric effects

- ionospheric effects

- tropospheric delays

- interference from external sources

- time to first fix (i.e., first position solution)

- interruption of the satellite signal due to blockage by terrain or manmade structures such as buildings and tunnels

- signal integrity and reacquisition capability

- reflected signals from buildings

Note that prior to the year 2000, a significant source of error for civilian-use GPS was Selective Availability, an intentional error induced by the U.S. DOD that degraded the user's navigation solution. An executive order by President Bill Clinton turned off Selective Availability permanently in the year 2000 [2-3, 5].

\subsection{GPS Map Databases}

Besides the determination of the vehicle position using GPS, the second key component in ground vehicle satellite-aided navigation is the digital road map, commonly provided on a CDROM. Two primary companies, Navteq and TeleAtlas, are developing digital road map databases for vehicle navigation and have extensive databases covering most of the United States, Canada, Europe, some countries in Asia and the Middle East, and other emerging markets worldwide. The accuracy of these databases, as determined by comparing road centerline vectors to ground truth, ranges from under 12 meters in urban areas to 50 meters or more in rural areas. New initiatives are underway to map road centerlines to better than 5 meter accuracies and to include vertical information for use in advanced driving systems. For more information on the use of vertical information in the GPS mapping, see below [2]. Some primary factors that influence the accuracy of the maps include the base map and photo material from which the digital map is produced [3]. 
Venhovens, et al. [3] refer to this digital cartography / GPS combination as an "electronic horizon of the vehicle" and they advocate that this is needed for the driver, and in the case of this thesis a predictive vehicle control system, to inform or react in advance to events which are either outside of the driver's visual range, or whose continuous monitoring would pose an unnecessary burden on the driver.

As pointed out by Kaplan, et al. [2] the process of correlating the vehicle path with a drivable path in the digital road map is called map matching. A basic assumption made is that the vehicle is on the road network so that the dead-reckoning (DR) position is constrained to one of the road segments in the map. The DR sensors, which provide a path of the vehicle, are combined with a robust map matching implementation that uses confidence measures to determine all possible road segments in the map on which the vehicle could be traveling. As the vehicle travels, distance traveled and changes in direction are used to continuously determine the shape of the route traveled; this shape is used to match the road network in the map through shape correlation. When an accurate heading is known, the list of roads is reduced to those that have a bearing within a tolerance of the vehicle heading. Through this process, the list of possible vehicle positions is eventually reduced to a unique segment and the confidence in the positioning solution increases accordingly. This process continues until there is only one possible vehicle position, at which point the map-matched position solution will have a small confidence region and can thus be considered highly reliable.

One concern is if the driver is on a road that is not in the map database. In order to support map matching, the map data should have high position accuracy, ideally better than 15 meters, to minimize incorrect road selections. The map data should be topologically correct, reflecting the real-world road network, so that the algorithm does not get confused if the user drives on a road that is not in the database. The expected accuracy of the road centerline data should be used in the map-matching process to determine the overall confidence region of the map-matched position solution.

Once a match is determined, the vehicle position is then displayed on the matched road segment. In traditional in-vehicle navigation systems this information would be used for the route guidance instructions, but in the case of the safety system presented in this thesis, this data would be fed into the predictive vehicle dynamics model. In this instance, the map would be used as a sensor itself to provide useful information to the positioning subsystem or to calibrate inertial and other DR sensors. These capabilities are broadly referred to as map aiding and map calibration [2].

Map aiding is most useful when map matching has determined that the vehicle has just turned a corner, in which case its position is in close proximity to the intersection of two streets of a known location in the map database. This reference position may be treated as a single position fix by the integration filter, which serves to correct or improve the accuracy of the absolute position determined by GPS. Further, if map matching has determined that the vehicle is traveling on a specific road in the database and that the road is straight, then a heading fix may be generated for the integration filter based on the bearing of that road segment according to the map database. One low cost navigation solution, proposed by Kaplan, et al., is map feedback. In this case a constraint is imposed on the model to force the heading of the vehicle to match the 
bearing of the road. This utilization of heading information could be used instead of DR sensors [2]. In order to be reliable, however, this system would have to assume that the digital maps being used were always correct and up-to-date.

In addition to the horizontal position components of road vectors, ground elevation data may be used to augment the performance of GPS. A digital terrain model (DTM) is a representation of the Earth's surface that can be used to extract elevation data. A digital elevation model (DEM) is a type of DTM with a regularly spaced grid of elevations corresponding to the elevation of the Earth's terrain at that point. Modern DTMs are derived from airborne or satellite-based remote sensors, are georeferenced using GPS coordinates, and have vertical accuracies better than 10 meters [2].

Terrain elevation can be used to improve the accuracy associated with GPS fixes for land applications. To apply a height constraint to a real-time Kalman filter, for example, an approximate or previous position can be used to extract the corresponding elevation from a DTM or DEM. Using a DEM would involve a simple value lookup and interpolation based on the coordinates and would therefore be easier from a computational perspective but would require a large amount of storage. On the other hand, a DTM that has the elevation data organized into vectors would use less storage but would require more involved computations to determine the elevation at a specific point. Another option would be for the elevation data to be integrated into the digital road maps as attribute data. This would simplify elevation lookup and keep storage requirements lower. According to Kaplan, et al., the vertical axis is the weakest part of the GPS solution, and terrain elevation data has not yet been widely used to augment GPS. This will likely change in navigation and driver safety systems once the elevation data is integrated into the digital road maps [2].

Map calibration is very similar to the process of using GPS data to calibrate inertial and other DR sensors. For example, a constant road heading may be used to calibrate a low-cost gyro or magnetic compass. In this case the gyro reading is then a direct measure of its bias. In the case of a vehicle making a turn at an intersection, the change in heading between the inbound and outbound segments can be used to calibrate a heading sensor. As Kaplan points out, however, with the current performance of GPS, map calibration of sensors is less common than it once was [2].

Since the GPS-aided navigation system relies on a digital map stored on a database, a significant concern arises regarding the accuracy of the map over time. For example the road geometry, construction, and even location, can change over time. Perhaps there is a new highway on- or off-ramp, or maybe a bridge is out. Up-to-the-minute cartographic material is crucial in providing viable, efficient navigation. According to the map compilers, $10-25 \%$ of the road segments will undergo changes every year. Therefore, map database updates for route guidance are made available to the end user on a quarterly basis [5]. An additional concern is if the enduser chooses not to update his map. For the purpose of this thesis, it is assumed that map databases are up to date. One idea for future implementation could be if the map database is stored on a hard drive in the vehicle instead of only on CDs, then it might be possible to have automatic updates via an internet connection similar to what is available now for anti-virus programs and Windows ${ }^{\mathrm{TM}}$ operating systems on personal computers. 
Several additional questions are:

Rather than relying on map CDs, would it be possible to use a system like Google $\operatorname{Earth}^{\mathrm{TM}}$ to provide the cartographic information? It would seem that since Google Earth ${ }^{\mathrm{TM}}$ is accessible through the internet this would require internet access all of the time. Beyond that, further research needs to be done.

The CD-based navigation system described here would require an in-vehicle navigation processing system similar to those in passenger cars with this feature. Further more, as pointed out above the digital maps need to be updated periodically, so how do these affect not only the initial cost of the vehicle but also the long-term operating cost, both for upkeep of the navigation system and the map updates? Since the intent of this paper is to describe the technology fusion of fuzzy logic and GPS-assisted guidance, cost is not a consideration. A future research step would be to implement this on a real vehicle and cost would be considered beyond that stage of development.

Regarding other traffic, Venhovens, et al. [3] has some description of this issue, but for the purpose of this thesis it is assumed no other traffic is present. Since there are currently some passenger vehicle navigation systems that have real-time traffic updates, future research will address this issue of implementing this feature.

\subsection{Feasibility for Automotive Applications}

As pointed out in the opening remarks of this paper, the intent of the GPS is to function as a prediction tool and ideally the vehicle shouldn't lose control. This might be true if everything worked properly all of the time, but this isn't always the case. It would be unsafe to require the safety critical electronic braking systems to rely on a GPS/INS input all of the time due to the potential for GPS dropouts or outages, or any of the other aforementioned reasons. Thus it should be made clear that the intention of this GPS assistance is just that: assistance. Beiker, et al. of BMW clearly explains that GPS assistance must only be used to enhance but not to ensure functionality of safety critical systems [6].

Beiker goes on to say that proof of feasibility for an automotive INS/GPS setup needs to cover two steps to check performance as well as practicability. First, the performance of the basic concept needs to be compared to a reference system to prove accuracy and reliability. Second, the proposed INS/GPS setup needs to be tested in various maneuvers and under various conditions to prove the practicability of such a system as well as its benefits for automotive applications.

The Beiker paper summarizes several dynamic tests performed on a real vehicle on different surfaces (i.e. dry concrete, snow, ice, etc) and compares the GPS/INS data to that obtained using an optical $5^{\text {th }}$ wheel, the Datron V Sensor from Corrsys, which they used as a reference for speed over ground and side slip angle. The maneuvers included a straight line ABS stop, a lane change maneuver, quasi-steady cornering, and sinusoidal steering. In all cases the GPS/INS system matched very closely with the optical $5^{\text {th }}$ wheel data and in one case, the sinusoidal steering, the 
GPS/INS was able to track the vehicle better than the optical unit as the tires reached their limits of adhesion.

In a second BMW paper authored by Venhovens, et al. [3], GPS was used to supplement several vehicle systems: Situation Adaptive Drivetrain Management (SAM), Adaptive Light Control (ALC), and Autonomous Speed Adaptation.

BMW's main objective of the drivetrain adaptation was to increase the fuel efficiency and cut the exhaust emissions by means of adapting the throttle, brake, and gears to the current traffic situation. The digitized road map and GPS navigation system are the basis for the Situation Adaptive Drivetrain Management (SAM). They give the location of relevant speed limits, traffic lights, junctions, bends, etc. Using the electronic horizon mentioned earlier along with the GPS and additional vehicle data, the driving situation several seconds ahead can be anticipated and if needed, possible interventions of the drivetrain can be selected [3].

For the Adaptive Light Control (ALC) system, the headlamps can be controlled using the vehicle trajectory prediction based on vehicle dynamics and/or route vectors from the navigation system. In the latter case the GPS system consistently keeps track of the precise location of the vehicle, and with its built-in digital road map has knowledge of the road characteristics ahead.

The BMW team found that in their tests with real vehicles and the standard GPS accuracy under 2-dimensional situations, the vehicle dynamics-based path prediction and the 2-dimensional navigation data used to control the headlamps was limited to certain road geometry. It was insufficient when encountering hilly roads, s-curves and other types of special road constructions. This was because the accuracy of the standard GPS and map databases was insufficient. Additional knowledge was necessary to overcome those deficiencies which led to the simulation and use of highly accurate DGPS and road databases. For this ALC system, the driving dynamic status of the car, coupled with the three-dimensional DGPS data via a Kalman filter and bi-splines, was used to derive the shape of the road [3].

In the case of the Autonomous Speed Adaptation, the maximum acceptable speed in turns was estimated based on the road geometry and the vehicle's dynamics. According to BMW, the three main parameters for curve speed estimation are the curve radius, the change of direction of a turn and the roadway type. Additional factors include surface quality, street width, number of lanes, shoulders, (daytime) visibility, weather (friction) and driving style of the driver. These could be superimposed on the calculated road speed, thus improving the safe curve speed estimate.

Most important from the feasibility standpoint of this thesis, the estimated safe speed is compared with the current vehicle speed. If the speed of travel is too high, either a warning is issued or automatic deceleration will take place [3]. This concept matches the design intent of the GPS-assisted vehicle predictive system presented in this thesis.

From the information presented here, it is clear that GPS and INS have been and can be used as inputs to existing automotive technology. In the vehicle dynamics maneuvers performed in [6]: straight line ABS stop, lane change maneuver, quasi-steady cornering, and sinusoidal steering, as well as the driver assistance systems described in [3]: Situation Adaptive Drivetrain Management 
(SAM), Adaptive Light Control (ALC), and Autonomous Speed Adaptation, the GPS and INS added significant value to the vehicle performance and safety.

A final, safety-related question is whether the driver would be able to override the system. For example, during an automatic deceleration, would the driver be able to override the system and either brake harder or perhaps not brake at all? As stated above, the GPS is merely a safety enhancement and the authors have not yet implemented the as-described system on a real vehicle. Therefore at this stage of development it is envisioned that the driver would have the ability to override the predictive control. 


\section{Chapter 4}

\section{Mathematical Modeling}

There are two main model types to mathematically represent a vehicle: the single track, or bicycle model, and the two track model. The bicycle model, while of reduced complexity compared to the two track model, has been shown to give satisfactory results for vehicle models that remain within the linear region of the tire [7-13]. The most common single track model contains two degrees of freedom (2DOF): Lateral Velocity and Yaw Rate. An extended bicycle model, like the one derived here, adds a Roll degree of freedom and thus becomes a three degrees of freedom (3DOF) model. Lateral Acceleration is a calculated value in addition to the matrix equation presented at the end of this chapter.

\subsection{Vehicle Coordinate Systems}

The vehicle coordinate system used is defined by SAE J670: Vehicle Dynamics Terminology, found in Appendix E of Dixon [7] and Appendix A of Gillespie [8].

Earth-Fixed Axis System (X, Y, Z) : This system is a right-hand orthogonal axis system fixed on the earth. The trajectory of the vehicle is described with respect to this earth-fixed axis system. The $\mathrm{X}$ - and $\mathrm{Y}$-axes are in a horizontal plane and the $\mathrm{Z}$-axis is directed downward.

Vehicle Axis System (x, y, z) : This system is a right-hand orthogonal axis system fixed in a vehicle such that with the vehicle moving steadily in a straight line on a level road, the $\mathrm{x}$-axis is substantially horizontal, points forward, and is in the longitudinal plane of symmetry. The y-axis points to the driver's right and the $\mathrm{z}$-axis points downward.

Angular Orientation : The orientation of the vehicle axis system $(x, y, z)$ with respect to the earthfixed axis system $(X, Y, Z)$ is given by a sequence of three angular rotations. The following sequence of rotations, starting from a condition in which the two sets of axes are initially aligned, is defined to be the standard:

(1) A yaw rotation, $\psi$, about the aligned $\mathrm{z}$ - and Z-axes.

(2) A pitch rotation, $\theta$, about the vehicle y-axis.

(3) A roll rotation, $\phi$, about the vehicle $x$-axis. 
Note 1: Angular rotations are positive clockwise when looking in the positive direction of the axis about which the rotation occurs.

Note 2: The order of rotations chosen for this thesis is Yaw-Roll-Pitch. See Section 4.4.

This coordinate system is illustrated below in Figure 4.1.

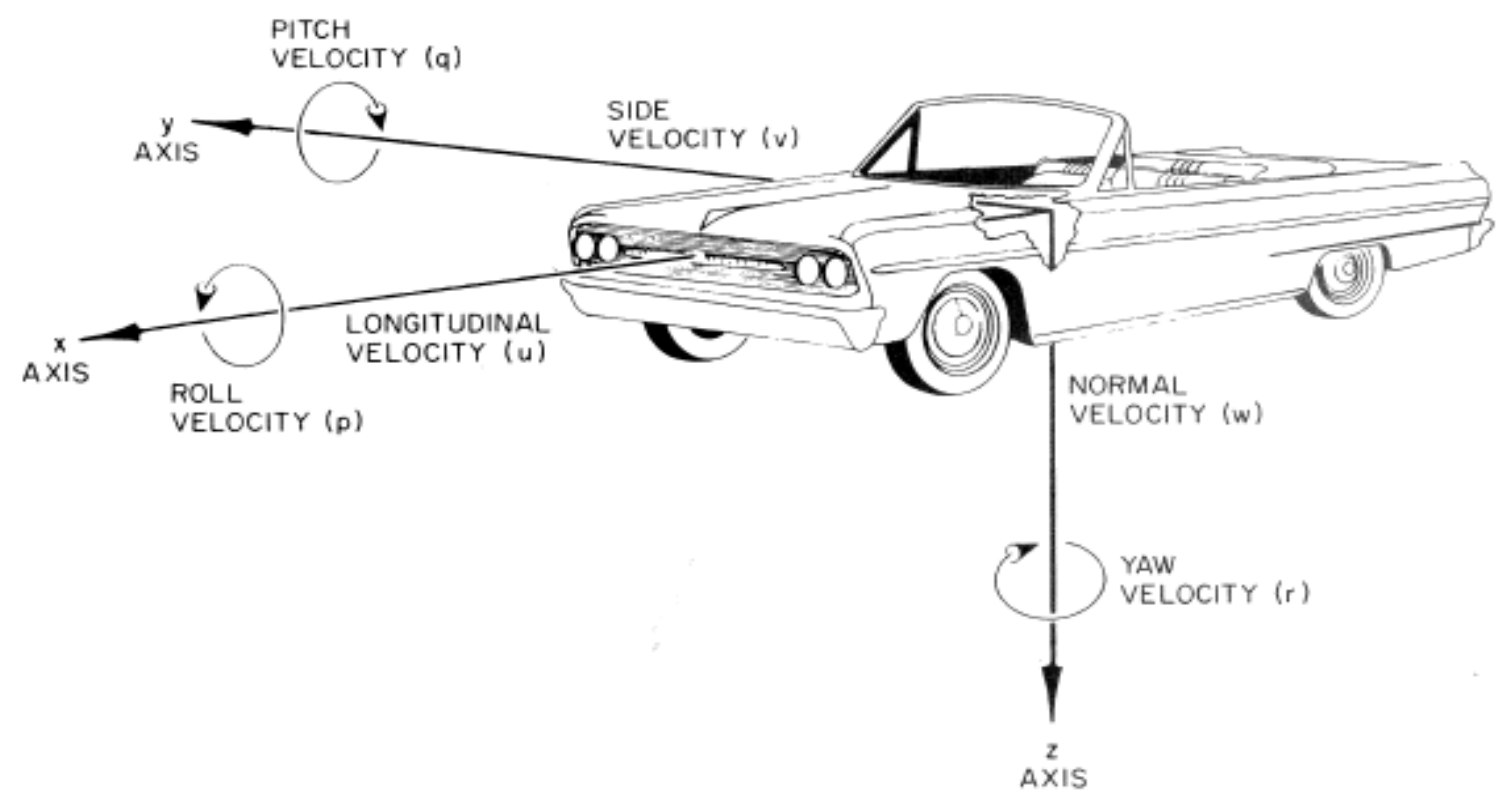

Figure 4.1. SAE Vehicle Coordinate System [7-8]

In a right turn the vehicle's yaw velocity, or yaw rate $r$, is positive and since the vehicle's sprung mass will roll to the outside of the turn, according to the SAE convention, the roll angle $\phi$ will be negative. See Figure 4.2, below.

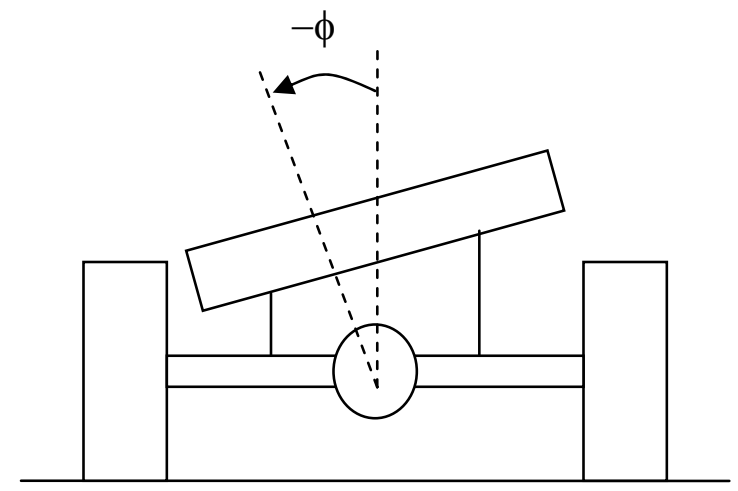

Figure 4.2: Simple vehicle model for roll in a right turn 
In Figure 4.2 the view is from the rear, looking forward, and the roll angle $\phi$ is exaggerated for clarity. Note that in this thesis the stiffness and damping properties of the suspension and tires are considered.

\subsection{Tire Slip Angle and Cornering Stiffness}

According to [7-13], under cornering conditions in which the tire must develop a lateral force, the tire will also experience lateral slip as it rolls. The angle between its direction of heading and its direction of travel of the center of tire-road contact is known as the tire slip angle, $\alpha$. See Figure 4.3.

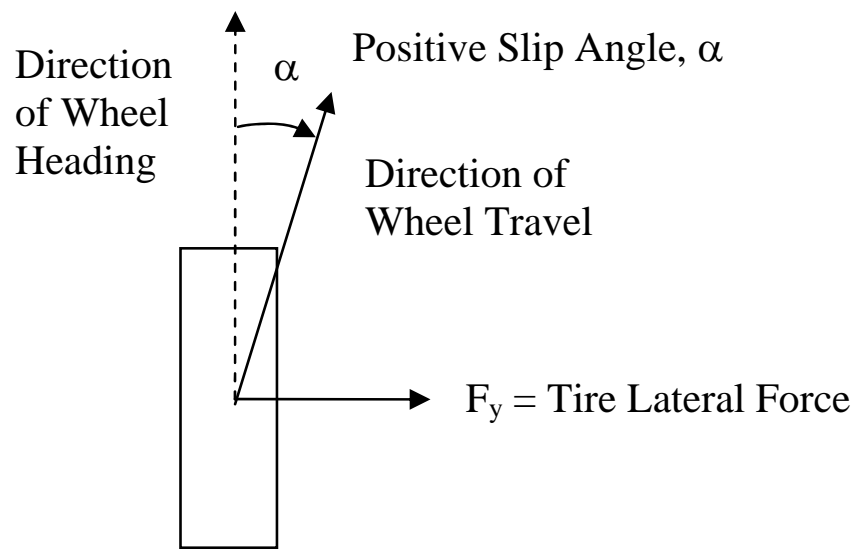

Figure 4.3: Top view of a tire illustrating the tire slip angle $\alpha$

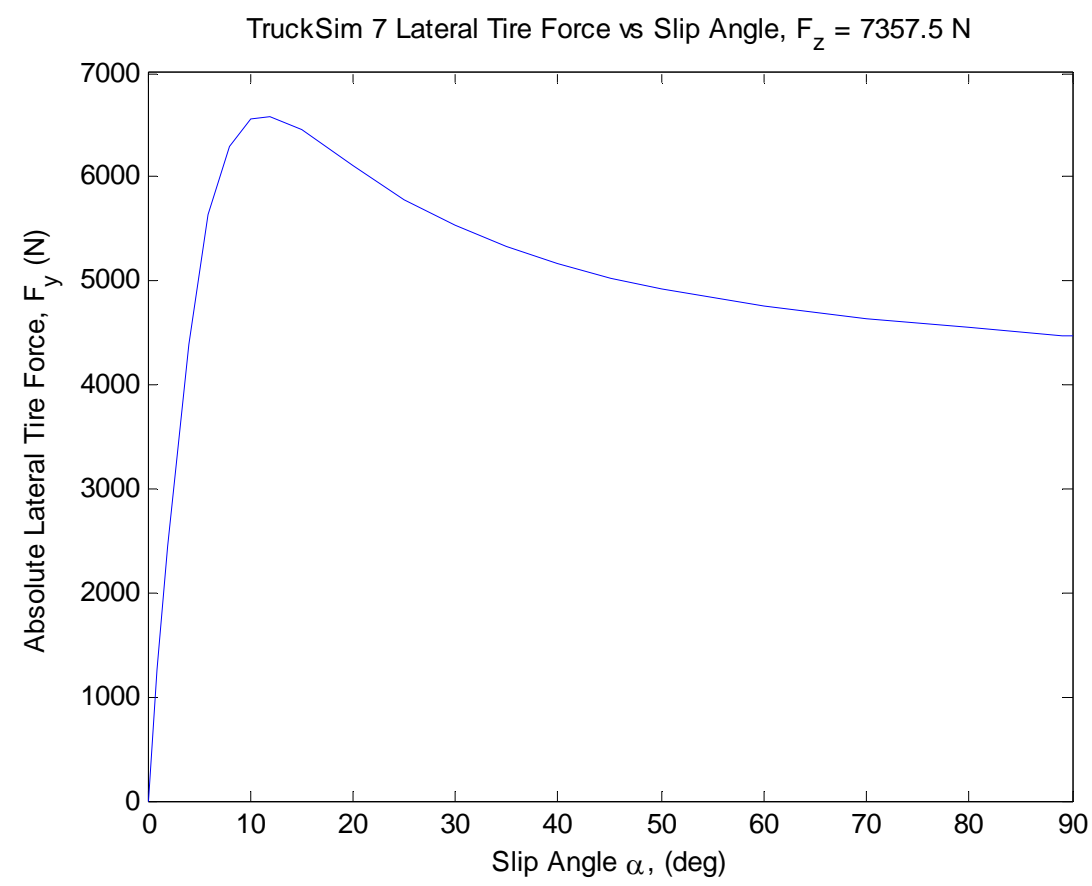

Figure 4.4: Absolute Lateral Tire Force vs. Slip Angle [31] 
A typical lateral force vs. slip angle curve for a truck tire is shown in Figure 4.4. This graph, from TruckSim 7 [31], shows the Absolute Lateral Tire Force $F_{y}$, measured in Newtons, vs. the Tire Slip Angle, $\alpha$, measured in degrees, for a slip angle range of zero to 90 degrees. The tire normal load is $F_{z}=7357.5$ Newtons.

At a given tire load the lateral force grows with slip angle. At low slip angles (5 degrees or less) the relationship is linear. Taking the slope of this linear region results in:

$$
C_{\alpha}=\frac{F_{y}}{\alpha}
$$

which has units of $\frac{l b_{f}}{\operatorname{deg}}$ or $\frac{N}{\operatorname{deg}}$.

This equation can be rearranged to solve for the lateral force $F_{y}$ :

$$
F_{y}=C_{\alpha} \alpha
$$

where the proportionality constant $C_{\alpha}$ is known as the cornering stiffness. The units are force $=\frac{\text { force }}{\text { angle }} *$ angle and this concept is illustrated in Figure 4.5.

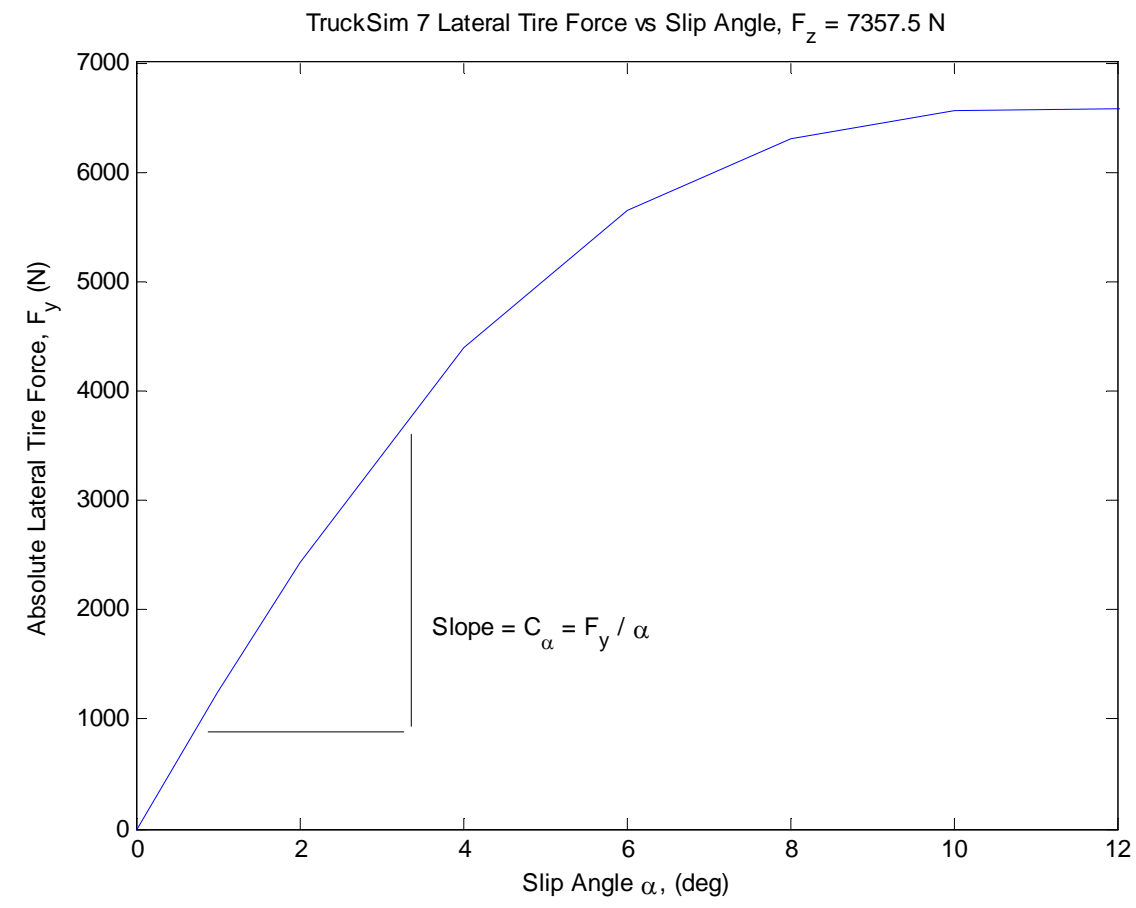

Figure 4.5: Absolute Lateral Tire Force vs. Slip Angle, showing linear tire properties [31]

As pointed out in [7-13], the cornering stiffness of a tire is dependent on many variables such as tire size, type (radial vs. bias ply construction), number of plies, cord angles, wheel width, and 
tread. For a given tire, the load and inflation pressure are the main variables. Speed does not strongly influence the cornering forces produced by the tire [7-13].

The front and rear tire slip angles can be derived in the following manner.

The front tire slip angle is defined graphically as:

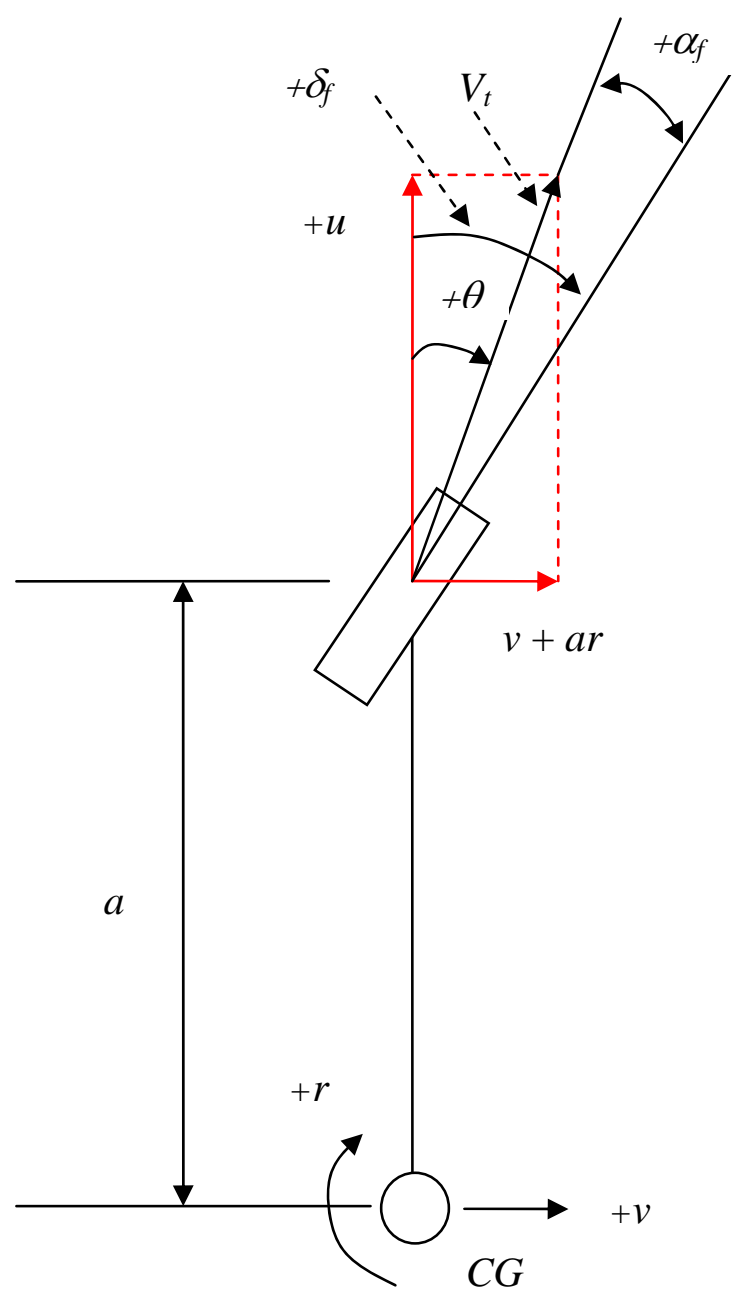

Figure 4.6: Front Tire Slip Angle

where:

$\alpha_{f}=$ front tire slip angle

$\delta_{f}=$ front wheel steer angle

$\theta=$ angle between the vehicle longitudinal velocity $u$ and the tire's tangential velocity $V_{t}$

$a=$ distance from vehicle CG to front axle center line

$C G=$ vehicle center of gravity

$r=$ vehicle yaw rate 
$u$ = longitudinal component of velocity vector

$v=$ lateral component of velocity vector

$V_{t}=$ tangential velocity of tire with respect to road radius. Vector velocity of $u$ and $(v+a r)$.

Looking at Figure 4.6 the front tire steer angle $\delta_{f}$ is shown to be:

$\delta_{f}=\theta+\alpha_{f}$

which can be rearranged to solve for the front tire slip angle $\alpha_{f}$ :

$\alpha_{f}=\delta_{f}-\theta$

Using Figure 4.6 above, or a simplified view shown in Figure 4.7, it can be seen that the angle $\theta$ is the inverse tangent of the lateral velocity component divided by the longitudinal velocity component:

$\theta=\tan ^{-1}\left(\frac{v+a r}{u}\right)$

where the quantity $(v+a r)$ is the total lateral velocity at the front tire center. The component $v$ comes from the lateral velocity of the vehicle and the product ar comes from the yaw rate $r$ multiplied by the distance $a$. This is the calculation for the angular velocity of the center of the front tire contact patch with respect to the vehicle CG.

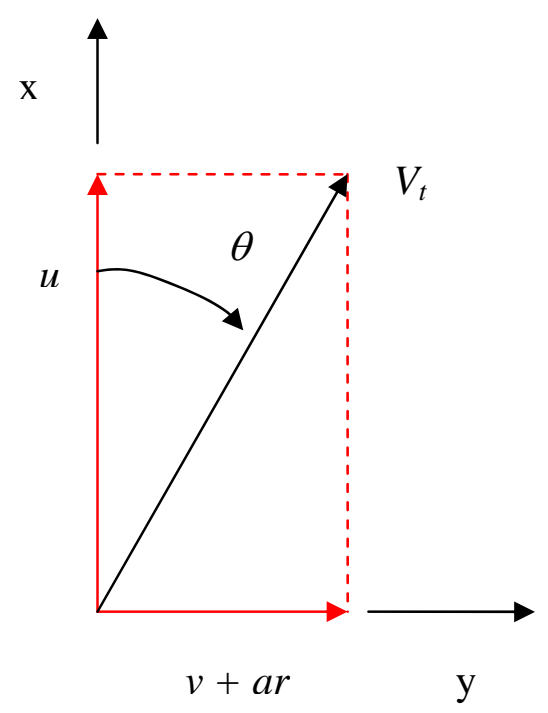

Figure 4.7: Simplified view of front tire velocities

For small angles $\theta \approx \tan ^{-1}(\theta)$. Substituting Eqn. 4.5 into Eqn. 4.4 the equation for the front tire slip angle becomes: 
$\alpha_{f}=\delta_{f}-\left(\frac{v+a r}{u}\right)$

The rear tire slip angle is defined graphically as:

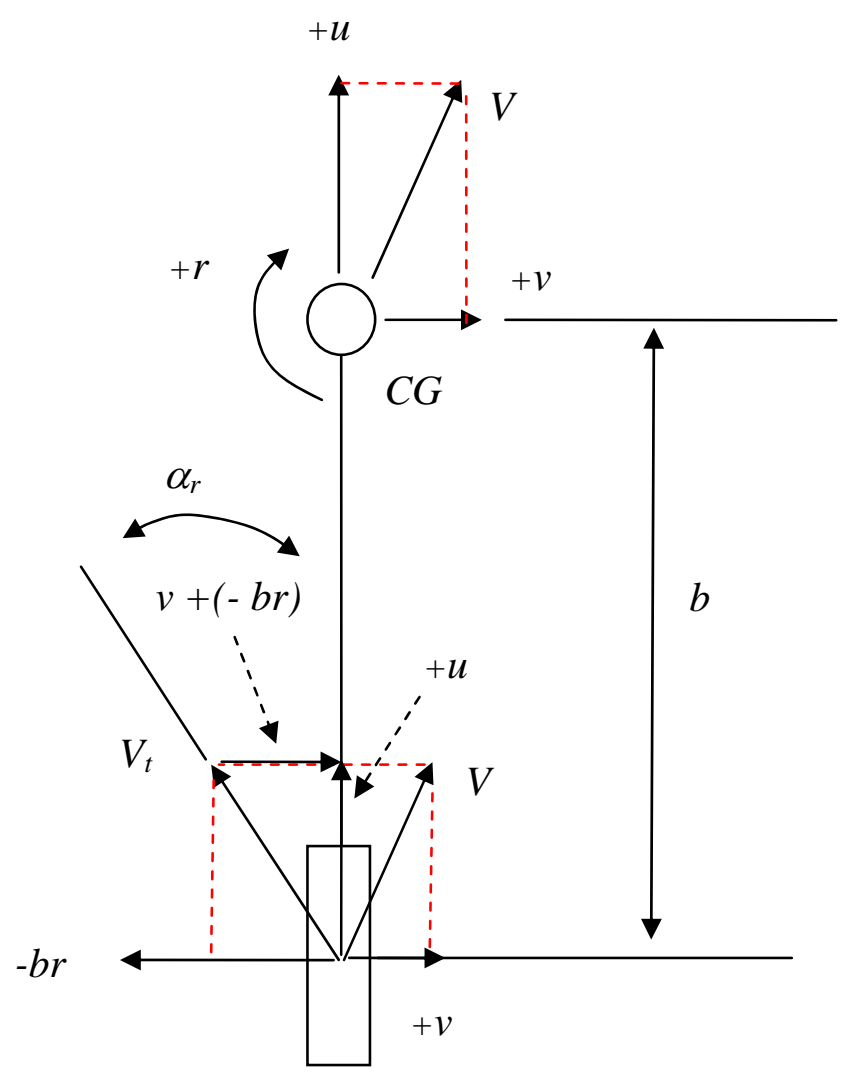

Figure 4.8: Rear Tire Slip Angle, first view

where:

$\alpha_{r}=$ rear tire slip angle

$C G=$ vehicle center of gravity

$b=$ distance from CG to rear axle

$r=$ vehicle yaw rate

$u=$ longitudinal component of velocity vector

$v=$ lateral component of velocity vector

$V=$ resulting vector velocity of $\mathrm{u}$ and $\mathrm{v}$

$V_{t}=$ tangential velocity of tire with respect to road radius. Vector velocity of $u$ and $-(v-b r)$.

In the same manner that the front tire slip angle was calculated, the total lateral velocity at the center of the rear tire contact patch is the quantity $v+(-b r)$ or $(v-b r)$. The component $v$ 
comes from the lateral velocity of the vehicle and the product - $b r$ comes from the yaw rate $r$ multiplied by the distance $b$. Again, this is the calculation for the angular velocity of the center of the rear tire contact patch with respect to the vehicle CG. Multiplying this quantity by a negative one to switch the direction of the $(v-b r)$ vector gives $-(v-b r)$. This is shown in Figure 4.9 below.

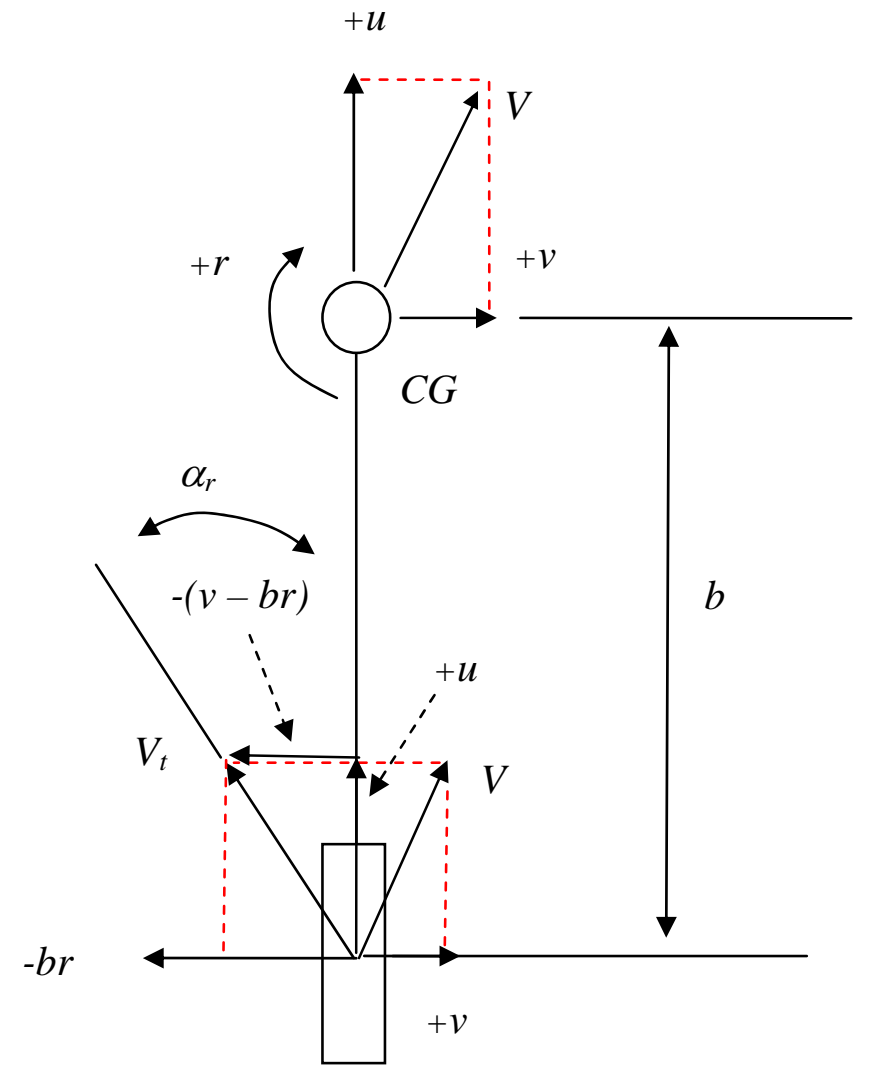

Figure 4.9: Rear Tire Slip Angle, second view

Looking at these figures it can be seen that the rear tire slip angle $\alpha_{r}$ is the inverse tangent of the lateral velocity component divided by the longitudinal velocity component:

$\alpha_{r}=\tan ^{-1}\left[-\left(\frac{v-b r}{u}\right)\right]$

which for small angles becomes:

$\alpha_{r}=-\left(\frac{v-b r}{u}\right)$ 


\subsection{Three Degree-of-Freedom (3DOF) Vehicle Dynamics Equation}

The main equation used in the Matlab simulations is a three degree-of-freedom (3DOF) equation containing lateral velocity, yaw rate, and roll. Lateral acceleration is calculated separately. A partial derivation is shown in [12] using Lagrange's equations. The Lagrange method is used here.

For a system with $n$ degrees of freedom, $n$ (generalized) coordinates $q_{i}$ are selected which are sufficient to completely describe the motion while possible kinematic constraints remain satisfied. The moving system possesses kinetic energy $T$ and potential energy $U$. External generalized forces $Q_{i}$ associated with the generalized coordinates $q_{i}$ may act on the system and do virtual work $\delta W$. The Lagrange equation for coordinate $q_{i}$ reads:

$$
\frac{d}{d t} \frac{\partial T}{\partial \dot{q}_{i}}-\frac{\partial T}{\partial q_{i}}+\frac{\partial U}{\partial q_{i}}=Q_{i}
$$

As will be shown later, the generalized coordinates will be $X, Y, \psi$, and $\phi$. It should be noted that the use of generalized coordinates allows for each coordinate to be defined with respect to the coordinate system that best defines that variable. For example, since the vehicle is translating in the Earth-fixed $X-Y$ plane, as is shown below in Figure 4.10, it makes sense to define the longitudinal and lateral vehicle velocities using the generalized coordinates $X$ and $Y$. However, with respect to the roll angle of the sprung mass, it will be shown that this angular displacement is the result of the longitudinal and lateral displacements, as well as the change in heading angle $\psi$. But, the angular displacement of the sprung mass takes place with respect to the chassis movements. It is therefore desirable to use the roll angle $\phi$ as the generalized coordinate, rather than attempt to define the roll angle of the sprung mass with respect to the Earth-fixed $X-Y$ coordinate system. Lagrange's equations were used since they allow this process.

As was stated above, generalized forces $Q_{i}$ are associated with generalized coordinates $q_{i}$. These in turn act on a system and do virtual work $\delta W$. Virtual work is the result since work $W$ can only happen with the passage of time. Since virtual displacements will be used, which assume infinitesimal displacements during which there is no passage of time, virtual work is the result.

It is important to note that the vehicle depicted in Figures 4.1, 4.2, and 4.10 performs a motion over a flat level road, i.e. no road bank angle is included in these equations. According to Pacejka [12], for motions near the $X$ axis and thus small yaw angles $\psi$, Eqn. 4.8 is adequate to derive the equations of motion. The relationship between the two sets of variables, i.e. the Earthfixed and vehicle-fixed coordinate systems, are shown graphically in Figure 4.10. 


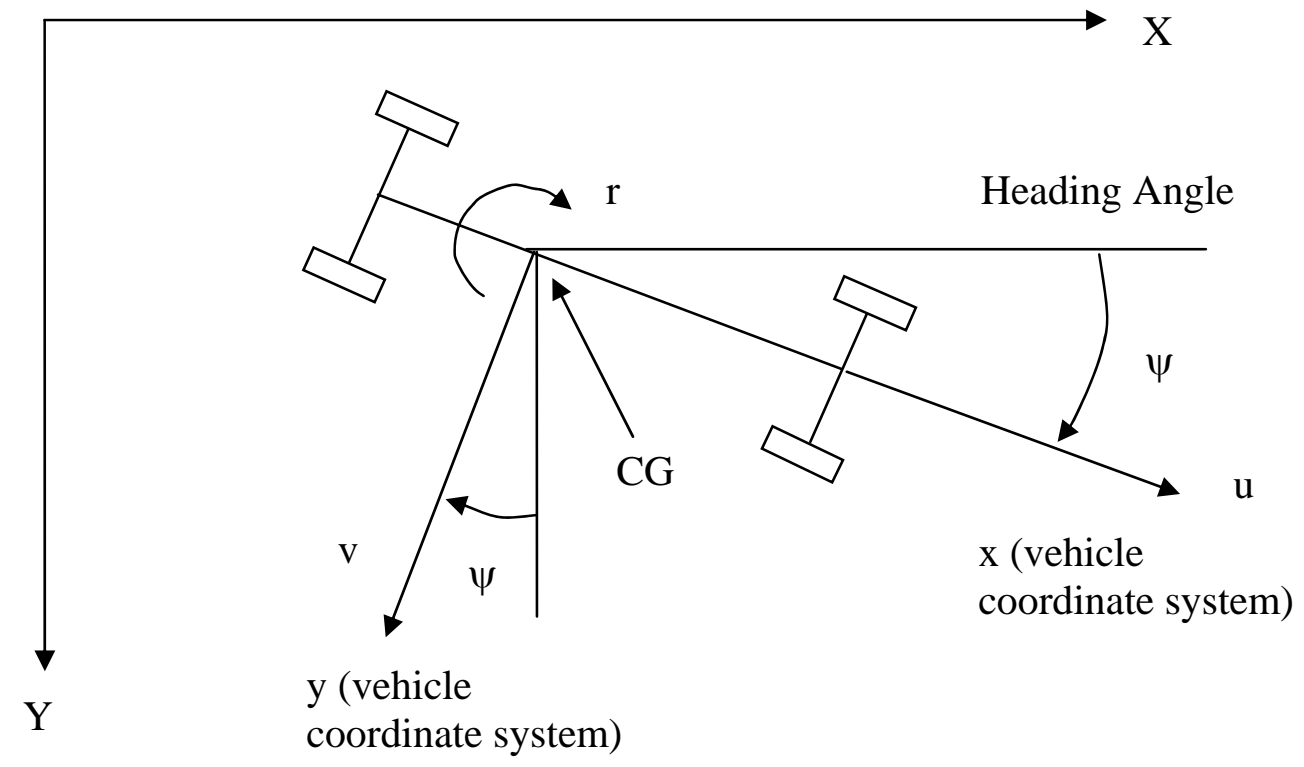

Figure 4.10: Top view of a generic vehicle driving straight but with a positive, non-zero heading angle $\psi$ with respect to the Earth-fixed coordinate system in the $X$ - $Y$ plane [8].

As can be seen in Figure 4.10, the vehicle is traveling in the $X-Y$ plane of the Earth-fixed coordinate system with a positive, non-zero heading angle $\psi$. The following equations can be derived for the longitudinal and lateral velocities of the vehicle in terms of the Earth-fixed coordinate system. If $X$ and $Y$ are the Earth-fixed position coordinates then $\dot{X}$ and $\dot{Y}$ are the Earth-fixed velocities, where the dots refer to differentiation with respect to time. Since the vehicle is translating in two dimensions, $X$ and $Y$, there are two components to each translational velocity term. The angular components come from the trigonometry of Figure 4.10 . The longitudinal velocity term $u$ is measured with respect to the $X$ axis. The velocity along the $X$ axis is positive:

$u_{X}=\dot{X} \cos \psi$

and the velocity along the $Y$ axis is also positive:

$u_{Y}=\dot{Y} \sin \psi$

The total expression is then the sum of these two velocities:

$u=u_{X}+u_{Y}=\dot{X} \cos \psi+\dot{Y} \sin \psi$

$u=\dot{X} \cos \psi+\dot{Y} \sin \psi$

The same methodology is used to derive the expression for the vehicle lateral velocity $v$. Measuring with respect to the $Y$ axis, the vehicle has a displacement in the negative $X$ direction: 
$v_{X}=-\dot{X} \sin \psi$

and positive in the $Y$ direction:

$v_{Y}=\dot{Y} \cos \psi$

The total expression is then the sum of these two velocities:

$v=v_{X}+v_{Y}=-\dot{X} \sin \psi+\dot{Y} \cos \psi$

$v=-\dot{X} \sin \psi+\dot{Y} \cos \psi$

Equation 4.11, below, indicates that the yaw rate $r$ is the time rate of change of the heading, or yaw, angle $\psi$.

$\dot{\psi}=r$

The next step is to prepare the first terms of equation 4.8 for the coordinates $X, Y$, and $\psi$ :

Solving for $\frac{\partial T}{\partial \dot{X}}$ :

$\frac{\partial u}{\partial \dot{X}}=\frac{\partial(\dot{X} \cos \psi+\dot{Y} \sin \psi)}{\partial \dot{X}}$

Since $\frac{\partial \dot{X}}{\partial \dot{X}}=1$

then $\frac{\partial(\dot{X} \cos \psi)}{\partial \dot{X}}=\cos \psi$

Since $\frac{\partial \dot{Y}}{\partial \dot{X}}=0$

then $\frac{\partial(\dot{Y} \sin \psi)}{\partial \dot{X}}=0$

Therefore $\frac{\partial u}{\partial \dot{X}}=\frac{\partial(\dot{X} \cos \psi+\dot{Y} \sin \psi)}{\partial \dot{X}}=\cos \psi+0=\cos \psi$

Carrying out the same procedure for $v=-\dot{X} \sin \psi+\dot{Y} \cos \psi$ : 
$\frac{\partial v}{\partial \dot{X}}=\frac{\partial(-\dot{X} \sin \psi+\dot{Y} \cos \psi)}{\partial \dot{X}}$

$\frac{\partial(-\dot{X} \sin \psi)}{\partial \dot{X}}=-\sin \psi$

$\frac{\partial(\dot{Y} \cos \psi)}{\partial \dot{X}}=0$

Therefore $\frac{\partial v}{\partial \dot{X}}=\frac{\partial(-\dot{X} \sin \psi+\dot{Y} \cos \psi)}{\partial \dot{X}}=-\sin \psi+0=-\sin \psi$

There is no $\dot{X}$ term in equation 4.11 so:

$\frac{\partial r}{\partial \dot{X}}=\frac{\partial \dot{\psi}}{\partial \dot{X}}=0$

Substituting these results into the first portion of equation 4.8, $\frac{\partial T}{\partial \dot{X}}$ using the chain rule yields:

$\frac{\partial T}{\partial \dot{X}}=\frac{\partial T}{\partial u} \frac{\partial u}{\partial \dot{X}}+\frac{\partial T}{\partial v} \frac{\partial v}{\partial \dot{X}}+\frac{\partial T}{\partial r} \frac{\partial r}{\partial \dot{X}}$

$\frac{\partial T}{\partial \dot{X}}=\frac{\partial T}{\partial u} \cos \psi-\frac{\partial T}{\partial v} \sin \psi+\frac{\partial T}{\partial r}(0)$

$\frac{\partial T}{\partial \dot{X}}=\frac{\partial T}{\partial u} \cos \psi-\frac{\partial T}{\partial v} \sin \psi$

Using the same methodology for $\frac{\partial T}{\partial \dot{Y}}$ :

$$
\begin{aligned}
& \frac{\partial u}{\partial \dot{Y}}=\frac{\partial(\dot{X} \cos \psi+\dot{Y} \sin \psi)}{\partial \dot{Y}}=0+\sin \psi=\sin \psi \\
& \frac{\partial v}{\partial \dot{Y}}=\frac{\partial(-\dot{X} \sin \psi+\dot{Y} \cos \psi)}{\partial \dot{Y}}=0+\cos \psi=\cos \psi
\end{aligned}
$$

There is no $\dot{Y}$ term in equation 4.11 so:

$\frac{\partial r}{\partial \dot{Y}}=\frac{\partial \dot{\psi}}{\partial \dot{Y}}=0$ 
Substituting these results into the first portion of equation 4.8, $\frac{\partial T}{\partial \dot{Y}}$ using the chain rule yields:

$\frac{\partial T}{\partial \dot{Y}}=\frac{\partial T}{\partial u} \frac{\partial u}{\partial \dot{Y}}+\frac{\partial T}{\partial v} \frac{\partial v}{\partial \dot{Y}}+\frac{\partial T}{\partial r} \frac{\partial r}{\partial \dot{Y}}$

$\frac{\partial T}{\partial \dot{Y}}=\frac{\partial T}{\partial u} \sin \psi+\frac{\partial T}{\partial v} \cos \psi+\frac{\partial T}{\partial r}(0)$

$\frac{\partial T}{\partial \dot{Y}}=\frac{\partial T}{\partial u} \sin \psi+\frac{\partial T}{\partial v} \cos \psi$

The next step is to find the partial derivative of the kinetic energy with respect to $\dot{\psi}$. There is no $\dot{\psi}$ term in either equations 4.9 c or 4.10 c so:

$\frac{\partial u}{\partial \dot{\psi}}=\frac{\partial(\dot{X} \cos \psi+\dot{Y} \sin \psi)}{\partial \dot{\psi}}=0$

$\frac{\partial v}{\partial \dot{\psi}}=\frac{\partial(-\dot{X} \sin \psi+\dot{Y} \cos \psi)}{\partial \dot{\psi}}=0$

$\frac{\partial r}{\partial \dot{\psi}}=\frac{\partial \dot{\psi}}{\partial \dot{\psi}}=1$

Substituting these results into the first portion of equation 4.8, $\frac{\partial T}{\partial \dot{\psi}}$ using the chain rule yields:

$\frac{\partial T}{\partial \dot{\psi}}=\frac{\partial T}{\partial u} \frac{\partial u}{\partial \dot{\psi}}+\frac{\partial T}{\partial v} \frac{\partial v}{\partial \dot{\psi}}+\frac{\partial T}{\partial r} \frac{\partial r}{\partial \dot{\psi}}$

$\frac{\partial T}{\partial \dot{\psi}}=\frac{\partial T}{\partial u}(0)+\frac{\partial T}{\partial v}(0)+\frac{\partial T}{\partial r}(1)$

$\frac{\partial T}{\partial \dot{\psi}}=\frac{\partial T}{\partial r}$

Finally the partial derivative of the kinetic energy with respect to $\psi$ is obtained:

$$
\begin{aligned}
& \frac{\partial u}{\partial \psi}=\frac{\partial(\dot{X} \cos \psi+\dot{Y} \sin \psi)}{\partial \psi}=-\dot{X} \sin \psi+\dot{Y} \cos \psi=v \\
& \frac{\partial v}{\partial \psi}=\frac{\partial(-\dot{X} \sin \psi+\dot{Y} \cos \psi)}{\partial \psi}=-\dot{X} \cos \psi-\dot{Y} \sin \psi=-(\dot{X} \cos \psi+\dot{Y} \sin \psi)=-u
\end{aligned}
$$




$$
\frac{\partial r}{\partial \psi}=\frac{\partial \dot{\psi}}{\partial \psi}=0
$$

Substituting these results into the first portion of equation 4.8, $\frac{\partial T}{\partial \psi}$ using the chain rule yields:

$$
\begin{aligned}
& \frac{\partial T}{\partial \psi}=\frac{\partial T}{\partial u}(-\dot{X} \sin \psi+\dot{Y} \cos \psi)+\frac{\partial T}{\partial v}[-(\dot{X} \cos \psi+\dot{Y} \sin \psi)]+\frac{\partial T}{\partial r}(0) \\
& \frac{\partial T}{\partial \psi}=\frac{\partial T}{\partial u} v-\frac{\partial T}{\partial v} u
\end{aligned}
$$

These equations can be renumbered as:

$$
\begin{aligned}
& \frac{\partial T}{\partial \dot{X}}=\frac{\partial T}{\partial u} \frac{\partial u}{\partial \dot{X}}+\frac{\partial T}{\partial v} \frac{\partial v}{\partial \dot{X}}=\frac{\partial T}{\partial u} \cos \psi-\frac{\partial T}{\partial v} \sin \psi \\
& \frac{\partial T}{\partial \dot{Y}}=\frac{\partial T}{\partial u} \frac{\partial u}{\partial \dot{Y}}+\frac{\partial T}{\partial v} \frac{\partial v}{\partial \dot{Y}}=\frac{\partial T}{\partial u} \sin \psi+\frac{\partial T}{\partial v} \cos \psi \\
& \frac{\partial T}{\partial \dot{\psi}}=\frac{\partial T}{\partial r} \\
& \frac{\partial T}{\partial \psi}=\frac{\partial T}{\partial u} v-\frac{\partial T}{\partial v} u
\end{aligned}
$$

The next step is to take the set of equations defined as equations $4.42-4.45$ and write them in the standard Lagrangian form of equation 4.8. As stated earlier the variables are $X, Y, \psi$, and $\phi$ so the generalized coordinates can be defined as:

$$
\begin{aligned}
& q_{1}=X \\
& q_{2}=Y \\
& q_{3}=\psi \\
& q_{4}=\phi
\end{aligned}
$$

Beginning with the generalized coordinate $q_{1}=X$ in the Lagrange equation:

$$
\frac{d}{d t}\left(\frac{\partial T}{\partial \dot{X}}\right)-\frac{\partial T}{\partial X}+\frac{\partial U}{\partial X}=Q_{X}
$$


From this equation the terms $\frac{\partial T}{\partial X}$ and $\frac{\partial U}{\partial X}$ can cancel because:

$\frac{\partial T}{\partial X}=0$ since kinetic energy is a function of velocity $\dot{X}$ and not position $X$.

$\frac{\partial U}{\partial X}=0$ since there is no potential energy in the $X$ direction.

Thus equation 4.50 becomes:

$\frac{d}{d t}\left(\frac{\partial T}{\partial \dot{X}}\right)=Q_{X}$

but from equation 4.42:

$\frac{\partial T}{\partial \dot{X}}=\frac{\partial T}{\partial u} \frac{\partial u}{\partial \dot{X}}+\frac{\partial T}{\partial v} \frac{\partial v}{\partial \dot{X}}=\frac{\partial T}{\partial u} \cos \psi-\frac{\partial T}{\partial v} \sin \psi$

Thus

$\frac{d}{d t}\left(\frac{\partial T}{\partial u} \frac{\partial u}{\partial \dot{X}}+\frac{\partial T}{\partial v} \frac{\partial v}{\partial \dot{X}}\right)=Q_{X}$

or

$\frac{d}{d t}\left(\frac{\partial T}{\partial u} \cos \psi-\frac{\partial T}{\partial v} \sin \psi\right)=Q_{X}$

Distributing the $\frac{d}{d t}$ yields:

$\frac{d}{d t}\left(\frac{\partial T}{\partial u} \cos \psi\right)-\frac{d}{d t}\left(\frac{\partial T}{\partial v} \sin \psi\right)=Q_{X}$

Using the product rule results in:

$\frac{d}{d t}\left(\frac{\partial T}{\partial u}\right) \cos \psi+\frac{\partial T}{\partial u}(-\sin \psi) \dot{\psi}-\frac{d}{d t}\left(\frac{\partial T}{\partial v}\right) \sin \psi-\frac{\partial T}{\partial v}(\cos \psi) \dot{\psi}=Q_{X}$ 
Collecting terms and grouping by $\cos \psi$ and $\sin \psi$ :

$\left(\frac{d}{d t} \frac{\partial T}{\partial u}-\frac{\partial T}{\partial v} \dot{\psi}\right) \cos \psi-\left(\frac{d}{d t} \frac{\partial T}{\partial v}+\frac{\partial T}{\partial u} \dot{\psi}\right) \sin \psi=Q_{X}$

The next step is to use the second generalized coordinate $q_{2}=Y$ :

$\frac{d}{d t}\left(\frac{\partial T}{\partial \dot{Y}}\right)-\frac{\partial T}{\partial Y}+\frac{\partial U}{\partial Y}=Q_{Y}$

Just as with equation 4.50 above the terms $\frac{\partial T}{\partial Y}$ and $\frac{\partial U}{\partial Y}$ can be cancelled because:

$\frac{\partial T}{\partial Y}=0$ since kinetic energy is a function of velocity $\dot{Y}$ and not position $Y$.

$\frac{\partial U}{\partial X}=0$ since there is no potential energy in the $Y$ direction.

Thus equation 4.56 becomes:

$\frac{d}{d t}\left(\frac{\partial T}{\partial \dot{Y}}\right)=Q_{Y}$

but from equation 4.43:

$\frac{\partial T}{\partial \dot{Y}}=\frac{\partial T}{\partial u} \frac{\partial u}{\partial \dot{Y}}+\frac{\partial T}{\partial v} \frac{\partial v}{\partial \dot{Y}}=\frac{\partial T}{\partial u} \sin \psi+\frac{\partial T}{\partial v} \cos \psi$

Thus equation 4.57 becomes:

$\frac{d}{d t}\left(\frac{\partial T}{\partial u} \frac{\partial u}{\partial \dot{Y}}+\frac{\partial T}{\partial v} \frac{\partial v}{\partial \dot{Y}}\right)=Q_{Y}$

or

$\frac{d}{d t}\left(\frac{\partial T}{\partial u} \sin \psi+\frac{\partial T}{\partial v} \cos \psi\right)=Q_{Y}$

Just as before the $\frac{d}{d t}$ will be distributed. Carrying out the product rule: 


$$
\begin{aligned}
& \frac{d}{d t}\left(\frac{\partial T}{\partial u} \sin \psi\right)+\frac{d}{d t}\left(\frac{\partial T}{\partial v} \cos \psi\right)=Q_{Y} \\
& \frac{d}{d t}\left(\frac{\partial T}{\partial u}\right) \sin \psi+\frac{\partial T}{\partial u}(\cos \psi) \dot{\psi}+\frac{d}{d t}\left(\frac{\partial T}{\partial v}\right) \cos \psi+\frac{\partial T}{\partial v}(-\sin \psi) \dot{\psi}=Q_{Y}
\end{aligned}
$$

Grouping like terms:

$$
\left(\frac{d}{d t} \frac{\partial T}{\partial u}-\frac{\partial T}{\partial v} \dot{\psi}\right) \sin \psi+\left(\frac{d}{d t} \frac{\partial T}{\partial v}+\frac{\partial T}{\partial u} \dot{\psi}\right) \cos \psi=Q_{Y}
$$

The next procedure consists of three major steps: multiply equation 4.55 by $\cos \psi$, multiply equation 4.61 by $\sin \psi$, and then add these two new equations together. This procedure will allow a modified Lagrange equation for the generalized force $Q_{u}$ to be written.

Multiplying equation 4.55 by $\cos \psi$ :

$$
\cos \psi\left[\left(\frac{d}{d t} \frac{\partial T}{\partial u}-\frac{\partial T}{\partial v} \dot{\psi}\right) \cos \psi-\left(\frac{d}{d t} \frac{\partial T}{\partial v}+\frac{\partial T}{\partial u} \dot{\psi}\right) \sin \psi\right]=Q_{X} \cos \psi
$$

Distributing the $\cos \psi$ :

$$
\left(\frac{d}{d t} \frac{\partial T}{\partial u}-\frac{\partial T}{\partial v} \dot{\psi}\right) \cos ^{2} \psi-\left(\frac{d}{d t} \frac{\partial T}{\partial v}+\frac{\partial T}{\partial u} \dot{\psi}\right) \sin \psi \cos \psi=Q_{X} \cos \psi
$$

Multiplying equation 4.61 by $\sin \psi$ :

$$
\sin \psi\left[\left(\frac{d}{d t} \frac{\partial T}{\partial u}-\frac{\partial T}{\partial v} \dot{\psi}\right) \sin \psi+\left(\frac{d}{d t} \frac{\partial T}{\partial v}+\frac{\partial T}{\partial u} \dot{\psi}\right) \cos \psi\right]=Q_{Y} \sin \psi
$$

Distributing the $\sin \psi$ :

$$
\left(\frac{d}{d t} \frac{\partial T}{\partial u}-\frac{\partial T}{\partial v} \dot{\psi}\right) \sin ^{2} \psi+\left(\frac{d}{d t} \frac{\partial T}{\partial v}+\frac{\partial T}{\partial u} \dot{\psi}\right) \sin \psi \cos \psi=Q_{Y} \sin \psi
$$


Adding these together:

$$
\begin{aligned}
& \left(\frac{d}{d t} \frac{\partial T}{\partial u}-\frac{\partial T}{\partial v} \dot{\psi}\right) \cos ^{2} \psi-\left(\frac{d}{d t} \frac{\partial T}{\partial v}+\frac{\partial T}{\partial u} \dot{\psi}\right) \sin \psi \cos \psi+\ldots \\
& \left(\frac{d}{d t} \frac{\partial T}{\partial u}-\frac{\partial T}{\partial v} \dot{\psi}\right) \sin ^{2} \psi+\left(\frac{d}{d t} \frac{\partial T}{\partial v}+\frac{\partial T}{\partial u} \dot{\psi}\right) \sin \psi \cos \psi=Q_{X} \cos \psi+Q_{Y} \sin \psi
\end{aligned}
$$

The second and fourth terms cancel due to having the same terms but opposite signs:

$$
\left(\frac{d}{d t} \frac{\partial T}{\partial u}-\frac{\partial T}{\partial v} \dot{\psi}\right) \cos ^{2} \psi+\left(\frac{d}{d t} \frac{\partial T}{\partial u}-\frac{\partial T}{\partial v} \dot{\psi}\right) \sin ^{2} \psi=Q_{X} \cos \psi+Q_{Y} \sin \psi
$$

Since the same terms are inside the parenthesis this equation can be rewritten as:

$$
\left[\frac{d}{d t} \frac{\partial T}{\partial u}-\frac{\partial T}{\partial v} \dot{\psi}\right]\left[\cos ^{2} \psi+\sin ^{2} \psi\right]=Q_{X} \cos \psi+Q_{Y} \sin \psi
$$

Using the trigonometric identity:

$$
\cos ^{2} \psi+\sin ^{2} \psi=1
$$

Equation 4.68 becomes:

$$
\frac{d}{d t} \frac{\partial T}{\partial u}-\frac{\partial T}{\partial v} \dot{\psi}=Q_{X} \cos \psi+Q_{Y} \sin \psi
$$

The next step is to define:

$Q_{X} \cos \psi+Q_{Y} \sin \psi=Q_{u}$

and recall equation 4.11:

$\dot{\psi}=r$

The result is the modified Lagrange equation:

$$
\frac{d}{d t} \frac{\partial T}{\partial u}-\frac{\partial T}{\partial v} r=Q_{u}
$$


A similar procedure will now be performed, but this time multiplying equation 4.55 by $\sin \psi$ and equation 4.61 by $(-\cos \psi)$. These two products are then added together. This operation will give a modified Lagrange equation for the generalized force $Q_{v}$.

Multiplying equation 4.55 by $\sin \psi$ :

$$
\sin \psi\left[\left(\frac{d}{d t} \frac{\partial T}{\partial u}-\frac{\partial T}{\partial v} \dot{\psi}\right) \cos \psi-\left(\frac{d}{d t} \frac{\partial T}{\partial v}+\frac{\partial T}{\partial u} \dot{\psi}\right) \sin \psi\right]=Q_{X} \sin \psi
$$

Distributing the $\sin \psi$ :

$$
\left(\frac{d}{d t} \frac{\partial T}{\partial u}-\frac{\partial T}{\partial v} \dot{\psi}\right) \sin \psi \cos \psi-\left(\frac{d}{d t} \frac{\partial T}{\partial v}+\frac{\partial T}{\partial u} \dot{\psi}\right) \sin ^{2} \psi=Q_{X} \sin \psi
$$

Multiplying equation 4.61 by $(-\cos \psi)$ :

$$
(-\cos \psi)\left[\left(\frac{d}{d t} \frac{\partial T}{\partial u}-\frac{\partial T}{\partial v} \dot{\psi}\right) \sin \psi+\left(\frac{d}{d t} \frac{\partial T}{\partial v}+\frac{\partial T}{\partial u} \dot{\psi}\right) \cos \psi\right]=Q_{Y}(-\cos \psi)
$$

Distributing the $(-\cos \psi)$ :

$$
\left(\frac{d}{d t} \frac{\partial T}{\partial u}-\frac{\partial T}{\partial v} \dot{\psi}\right) \sin \psi(-\cos \psi)-\left(\frac{d}{d t} \frac{\partial T}{\partial v}+\frac{\partial T}{\partial u} \dot{\psi}\right) \cos ^{2} \psi=Q_{Y}(-\cos \psi)
$$

Adding these together:

$$
\begin{aligned}
& \left(\frac{d}{d t} \frac{\partial T}{\partial u}-\frac{\partial T}{\partial v} \dot{\psi}\right) \sin \psi \cos \psi-\left(\frac{d}{d t} \frac{\partial T}{\partial v}+\frac{\partial T}{\partial u} \dot{\psi}\right) \sin ^{2} \psi-\ldots \\
& \left(\frac{d}{d t} \frac{\partial T}{\partial u}-\frac{\partial T}{\partial v} \dot{\psi}\right) \sin \psi \cos \psi-\left(\frac{d}{d t} \frac{\partial T}{\partial v}+\frac{\partial T}{\partial u} \dot{\psi}\right) \cos ^{2} \psi=Q_{X} \sin \psi-Q_{Y} \cos \psi
\end{aligned}
$$

It can be seen that the first and third terms cancel because they contain the same components but opposite sign. After cancelling these terms the resulting equation is:

$$
-\left(\frac{d}{d t} \frac{\partial T}{\partial v}+\frac{\partial T}{\partial u} \dot{\psi}\right) \sin ^{2} \psi-\left(\frac{d}{d t} \frac{\partial T}{\partial v}+\frac{\partial T}{\partial u} \dot{\psi}\right) \cos ^{2} \psi=Q_{X} \sin \psi-Q_{Y} \cos \psi
$$


Several things will allow the simplification of this equation. First, both sets of terms on the left side are preceded by a negative $\left(\frac{d}{d t} \frac{\partial T}{\partial v}+\frac{\partial T}{\partial u} \dot{\psi}\right)$. Therefore those can be factored out. Second, it can be seen that, just as in equation 4.68, there is a $\sin ^{2} \psi$ and a $\cos ^{2} \psi$. Using the trigonometric identity of equation $4.69, \cos ^{2} \psi+\sin ^{2} \psi=1$, those terms can be factored out too. This leaves:

$$
-\left[\left(\frac{d}{d t} \frac{\partial T}{\partial v}+\frac{\partial T}{\partial u} \dot{\psi}\right)\left[\sin ^{2} \psi+\cos ^{2} \psi\right]\right]=Q_{X} \sin \psi-Q_{Y} \cos \psi
$$

Equation 4.79 simplifies to:

$$
-\left[\frac{d}{d t} \frac{\partial T}{\partial v}+\frac{\partial T}{\partial u} \dot{\psi}\right]=Q_{X} \sin \psi-Q_{Y} \cos \psi
$$

Multiplying through by a (-1), equation 4.80 becomes:

$$
\left[\frac{d}{d t} \frac{\partial T}{\partial v}+\frac{\partial T}{\partial u} \dot{\psi}\right]=-Q_{X} \sin \psi+Q_{Y} \cos \psi
$$

where $Q_{v}$ is defined to be:

$$
-Q_{X} \sin \psi+Q_{Y} \cos \psi=Q_{v}
$$

Using equation 4.11 to make the final substitution:

$$
\dot{\psi}=r
$$

Equation 4.82 becomes:

$$
\frac{d}{d t} \frac{\partial T}{\partial v}+\frac{\partial T}{\partial u} r=Q_{v}
$$

To find the third modified Lagrange equation for the generalized coordinate $q_{3}=\psi$, equation 4.8 is again written but now in terms of $q_{3}=\psi$ :

$$
\frac{d}{d t} \frac{\partial T}{\partial \dot{\psi}}-\frac{\partial T}{\partial \psi}+\frac{\partial U}{\partial \psi}=Q_{\psi}
$$

The term $\frac{\partial U}{\partial \psi}$ is going to be cancelled since it is assumed that the vehicle is traveling on a level road and there is no potential energy associated with the yaw angle. 
Thus equation 4.84 becomes:

$\frac{d}{d t} \frac{\partial T}{\partial \dot{\psi}}-\frac{\partial T}{\partial \psi}=Q_{\psi}$

Writing:

$\frac{\partial T}{\partial \dot{\psi}}=\frac{\partial T}{\partial r}$

and from equation 4.41:

$\frac{\partial T}{\partial \psi}=\frac{\partial T}{\partial u} v-\frac{\partial T}{\partial v} u$

Equation 4.85 becomes:

$\frac{d}{d t} \frac{\partial T}{\partial r}-\left[\frac{\partial T}{\partial u} v-\frac{\partial T}{\partial v} u\right]=Q_{\psi}$

Distributing the negative sign:

$\frac{d}{d t} \frac{\partial T}{\partial r}-v \frac{\partial T}{\partial u}+u \frac{\partial T}{\partial v}=Q_{\psi}$

Finally, there is both kinetic and potential energy associated with the roll angle $\phi$, i.e. the vehicle will have its maximum potential energy in roll when there is zero roll angle (body in its upright, non-rolled position). The vehicle is not a rigid body and translates in the Earth-fixed $X-Y$ coordinate system. Since kinetic energy is dependent on velocity and not position, as the vehicle translates in this $X-Y$ coordinate system, its coordinates at an initial time $\mathrm{t}_{1}$ are not the same as at a later time $t_{2}$. In order for the vehicle to change its position it has to execute a change in yaw angle $\psi$ and yaw angular rate $r$. The combination of the yaw angle $\psi$, the translational motion, and the non-rigid vehicle results in the roll angle $\phi$ and roll angle rate $\dot{\phi}$. Direct application of equation 4.8 can be used to obtain equation 4.89 . Using the generalized coordinate $q_{4}=\phi$ :

$\frac{d}{d t} \frac{\partial T}{\partial \dot{\phi}}-\frac{\partial T}{\partial \phi}+\frac{\partial U}{\partial \phi}=Q_{\phi}$

These equations can be renumbered equations $4.90-4.93$ :

$\frac{d}{d t} \frac{\partial T}{\partial u}-r \frac{\partial T}{\partial v}=Q_{u}$ 
$\frac{d}{d t} \frac{\partial T}{\partial v}+r \frac{\partial T}{\partial u}=Q_{v}$

$\frac{d}{d t} \frac{\partial T}{\partial r}-v \frac{\partial T}{\partial u}+u \frac{\partial T}{\partial v}=Q_{\psi}$

$\frac{d}{d t} \frac{\partial T}{\partial \dot{\phi}}-\frac{\partial T}{\partial \phi}+\frac{\partial U}{\partial \phi}=Q_{\phi}$

The generalized forces are found from the virtual work:

$\delta W=\sum_{j=1}^{4} Q_{j} \delta q_{j}$

with $q_{j}$ referring to the quasi coordinates $x$ and $y$, and the coordinates $\psi$ and $\phi$ [12]. For the vehicle model of Figures 4.1, 4.2, 4.10, and 4.11 the virtual work is found as a result of the virtual displacements $\delta x, \delta y, \delta \psi$, and $\delta \phi$ (NOTE: these $\delta$ are not steer angles):

$\delta W=\sum F_{x} \delta x+\sum F_{y} \delta y+\sum M_{z} \delta \psi+\sum M_{\phi} \delta \phi$

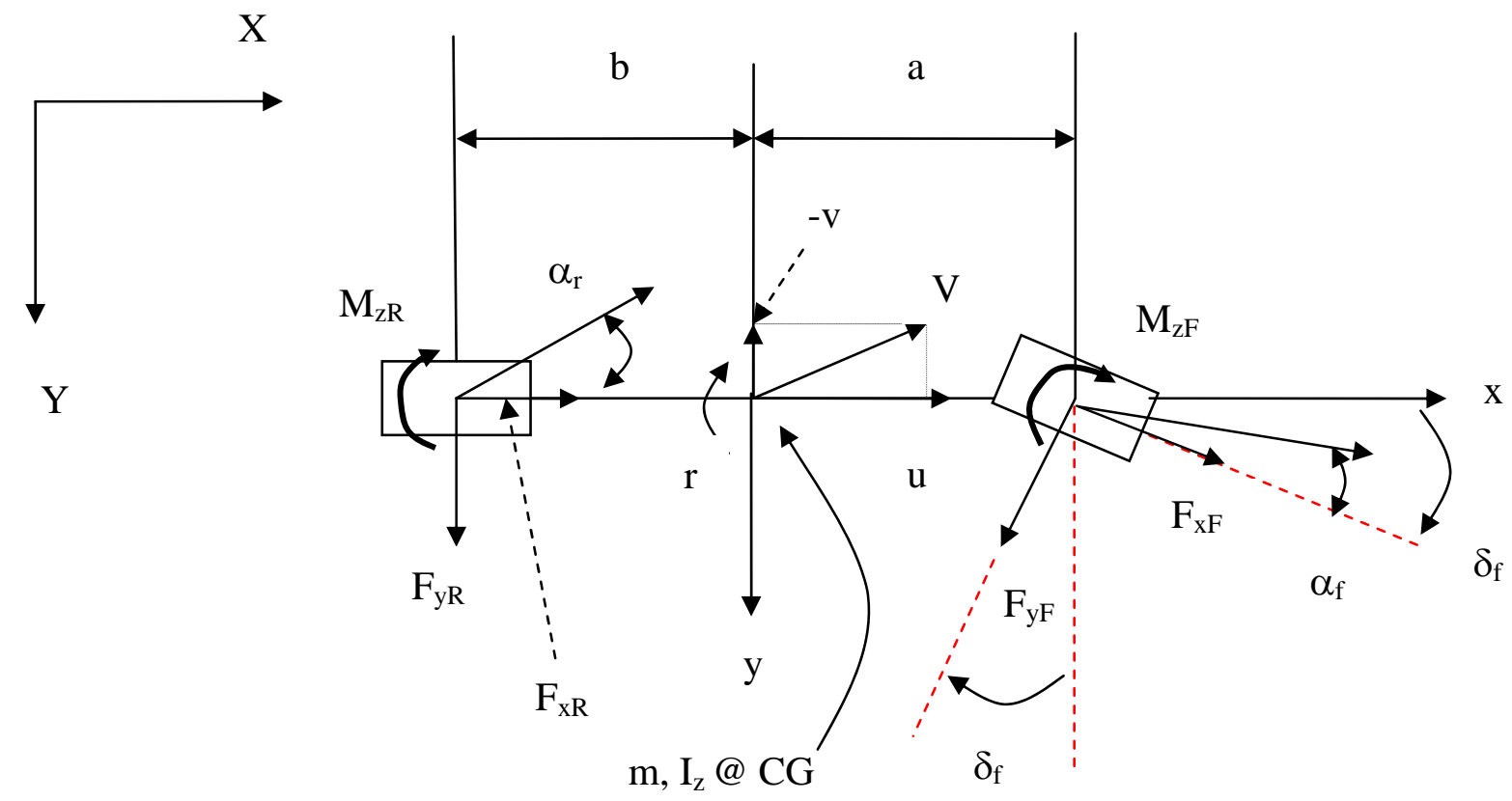

Figure 4.11: Simple car model with side force characteristics for front and rear (driven) axle, yaw plane [12]

Looking at Figure 4.11, the equations for the sum of the forces in the $\mathrm{x}$ and $\mathrm{y}$ directions as well as the moments about the $\mathrm{z}$ axis can be written. The following figure is helpful in writing the force equations: 


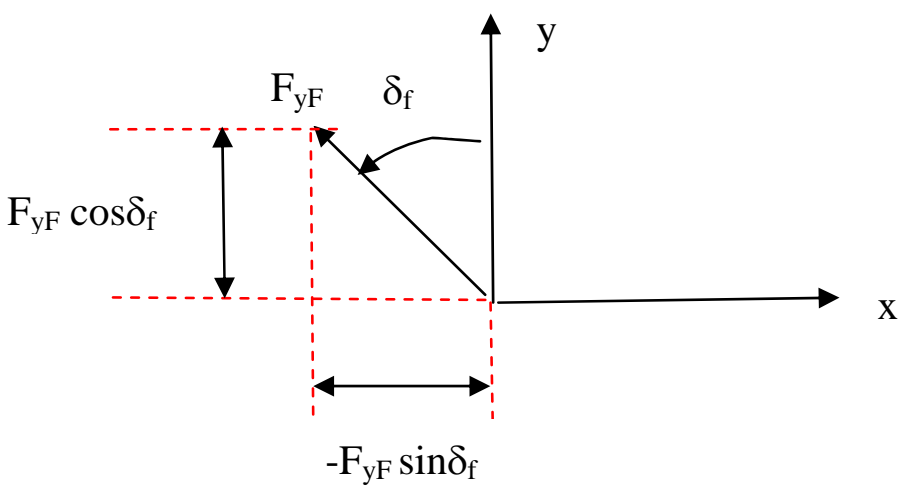

Figure 4.12: Visual aid for writing the force equations

$$
\begin{aligned}
& Q_{u}=\sum F_{x}=F_{x F} \cos \delta_{f}-F_{y F} \sin \delta_{f}+F_{x R} \\
& Q_{v}=\sum F_{y}=F_{x F} \sin \delta_{f}+F_{y F} \cos \delta_{f}+F_{y R} \\
& Q_{\psi}=\sum M_{z}=a F_{x F} \sin \delta_{f}+a F_{y F} \cos \delta_{f}-b F_{y R}+M_{z F}+M_{z R}
\end{aligned}
$$

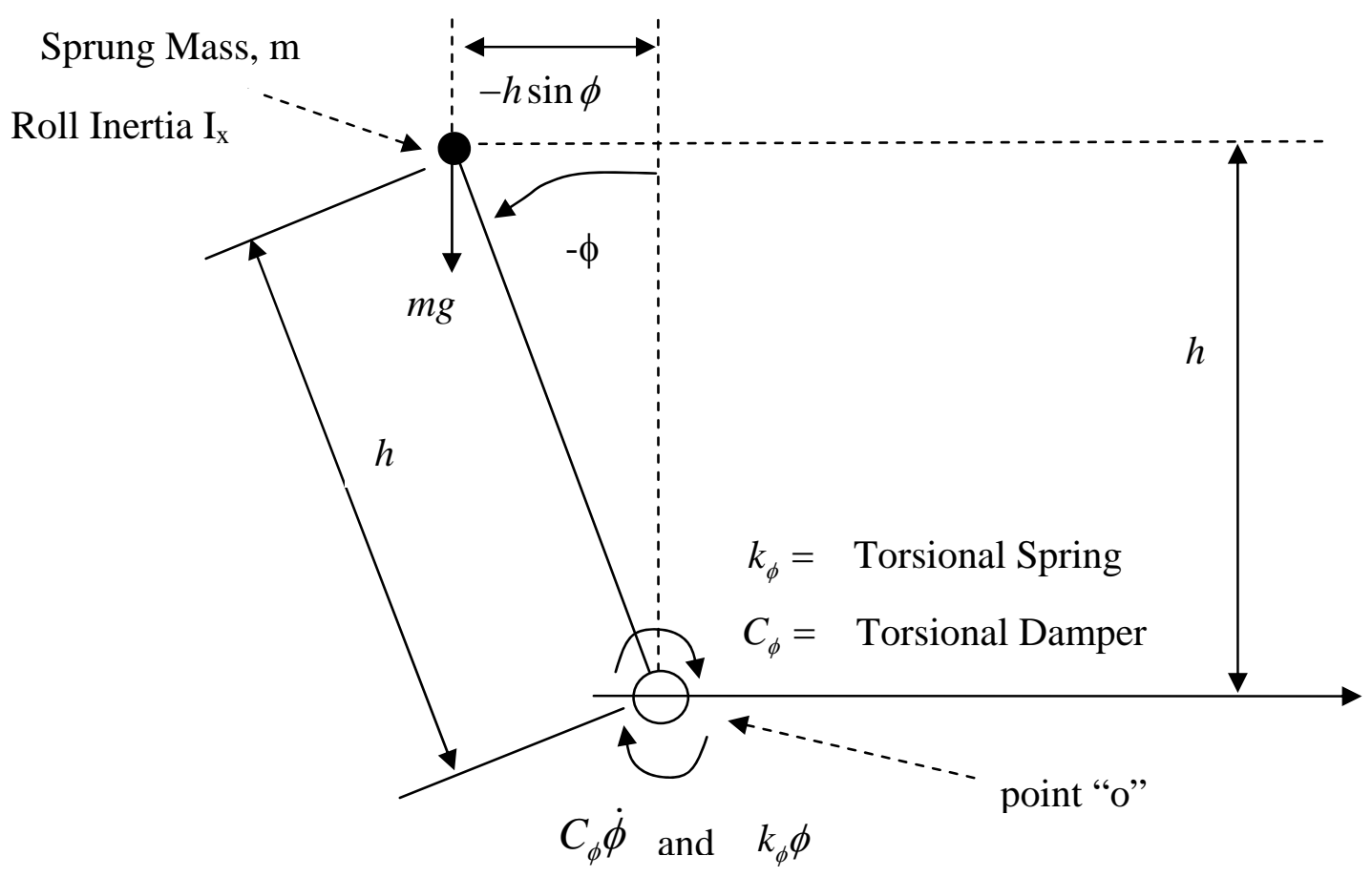

Figure 4.13: Simple schematic illustrating the roll of the sprung mass, $\mathrm{m}$. This roll motion is resisted by a torsional spring and damper, which on the vehicle includes the suspension springs and dampers, anti-roll bars, and tires. It should be noted that this view is from the rear, looking forward, while the vehicle is executing a right turn. This is consistent with Figure 4.2, above. 
It is also important to note that the sprung mass CG height is considered to be equal to the vehicle CG height; thus, the variable $h$ represents both dimensions and the sprung mass / CG rotates about a point on the ground, not a separate roll axis. This was done for simplicity and because this variable was readily available in TruckSim 7, the Mechanical Simulation Corp. simulation program on which the Matlab vehicle was modeled. It was possible to define the coordinate systems in TruckSim 7 such that the sprung mass height was equal to the unladen CG height. A literature search indicated that for some vehicles the sprung mass height is very close to the vehicle CG height [15].

As a vehicle is loaded with cargo and as it performs dynamic maneuvers the actual CG location varies, but since small angles are assumed this change should be small.

If the springs and anti-roll bars of the suspension can be represented by a torsional spring the equation of such a spring is:

$$
\frac{\partial U}{\partial \phi}=k_{\phi} \phi
$$

This can be rearranged to read:

$\partial U=k_{\phi} \phi \partial \phi$

which can then be integrated to find the potential energy of a torsional spring:

$\int \partial U=\int k_{\phi} \phi \partial \phi$

This results in:

$U=\frac{1}{2} k_{\phi} \phi^{2}$

The potential energy for the sprung mass is now written. Using Figure 4.13 it can be seen that:

$$
\frac{\partial U}{\partial \phi}=m g(-h \sin \phi)=-m g h \sin \phi
$$

The term $(-h \sin \phi)$ includes a negative sign due to: (1) The SAE convention of having the positive lateral direction to the right, i.e. out the passenger side of the vehicle, and (2) As a vehicle executes a right turn the sprung mass will roll to the left. This left rolling results in the sprung mass having a small horizontal displacement in the negative lateral direction.

Just as was done with the torsional spring, equation 4.103 can be rewritten as:

$$
\partial U=-m g h \sin \phi \partial \phi
$$


and assuming small angles $\sin \phi$ becomes $\phi$.

Integrating equation 4.104:

$\int \partial U=\int-m g h \phi \partial \phi$

results in:

$U=-m g h\left[\frac{1}{2} \phi^{2}\right]$

or:

$U=-\frac{1}{2} m g h \phi^{2}$

Equations 4.102 and 4.106 can be combined to represent the total potential energy of the sprung mass and suspension represented by a torsional spring:

$U=\frac{1}{2} k_{\phi} \phi^{2}-\frac{1}{2} m g h \phi^{2}$

Using Figure 4.13 to take moments about point "o" to obtain the roll moment equation:

$\sum M_{o}=-I_{\chi} \ddot{\phi}=C_{\phi} \dot{\phi}+k_{\phi} \phi-m g h \sin \phi$

But, the roll inertia $I_{x}$ can be defined as:

$I_{x}=m h^{2}$

Thus equation 4.108 becomes:

$\sum M_{o}=-m h^{2} \ddot{\phi}=C_{\phi} \dot{\phi}+k_{\phi} \phi-m g h \sin \phi$

which can also be written as:

$m h^{2} \ddot{\phi}+C_{\phi} \dot{\phi}+k_{\phi} \phi-m g h \sin \phi=0$

Since damping is a non-conservative force and is therefore the work of an external force, equation 4.111 can be rewritten as (i.e. the potential energy terms are conservative forces and therefore cancel): 
$m h^{2} \ddot{\phi}=-C_{\phi} \dot{\phi}$

which becomes:

$\sum M_{\phi}=-C_{\phi} \dot{\phi}$

Assuming small angles for steering $\delta_{f}$ :

$\sin \delta_{f} \approx \delta_{f}$

$\cos \delta_{f} \approx 1$

and using Figure 4.13 the above equations become:

$Q_{u}=\sum F_{x}=F_{x F}(1)-F_{y F} \delta_{f}+F_{x R}$

$Q_{u}=\sum F_{x}=F_{x F}-F_{y F} \delta_{f}+F_{x R}$

$Q_{v}=\sum F_{y}=F_{x F} \delta_{f}+F_{y F}(1)+F_{y R}$

$Q_{v}=\sum F_{y}=F_{x F} \delta_{f}+F_{y F}+F_{y R}$

$Q_{\psi}=\sum M_{z}=a F_{x F} \delta_{f}+a F_{y F}(1)-b F_{y R}+M_{z F}+M_{z R}$

$Q_{\psi}=\sum M_{z}=a F_{x F} \delta_{f}+a F_{y F}-b F_{y R}+M_{z F}+M_{z R}$

$Q_{\phi}=\sum M_{\phi}=-C_{\phi} \dot{\phi}$

The longitudinal forces are assumed to be the same for the left and right wheels, and the effect of additional steer angles are neglected here (i.e. no rear wheel steering).

\subsection{Rotation Matrices, Angular Velocities, and the Final Equation}

From [14], for successive rotations about each of three axes i, j, and k, there are six possible orders of such a rotation:

$1-2-3 \quad 2-1-3 \quad 3-1-2$

$1-3-2 \quad 2-3-1 \quad 3-2-1$

For the purpose of this thesis these are referred to as Type I Rotations:

$1-2-3=\phi-\theta-\psi \quad$ Roll - Pitch - Yaw

$1-3-2=\phi-\psi-\theta$ Roll - Yaw - Pitch 
$2-1-3=\theta-\phi-\psi \quad$ Pitch - Roll - Yaw

$2-3-1=\theta-\psi-\phi \quad$ Pitch - Yaw - Roll

$3-1-2=\psi-\phi-\theta \quad$ Yaw - Roll - Pitch

$3-2-1=\psi-\theta-\phi \quad$ Yaw - Pitch - Roll

First and third rotations about the same axis with the second rotation about one of the two remaining axes yields these six possibilities:

$1-2-1 \quad 2-1-2 \quad 3-1-3$

$1-3-1 \quad 2-3-2 \quad 3-2-3$

For the purpose of this thesis these are referred to as Type II Rotations:

$1-2-1=\phi-\theta-\phi \quad$ Roll - Pitch - Roll

$1-3-1=\phi-\psi-\phi \quad$ Roll - Yaw - Roll

$2-1-2=\theta-\phi-\theta \quad$ Pitch - Roll - Pitch

$2-3-2=\theta-\psi-\theta$ Pitch - Yaw - Pitch

$3-1-3=\psi-\phi-\psi$ Yaw - Roll - Yaw

$3-2-3=\psi-\theta-\psi \quad$ Yaw - Pitch - Yaw

The second type of rotation sequences can be useful in special situations and sometimes helps to solve problems in which successive rotations about three distinct axes may give rise to singularities.

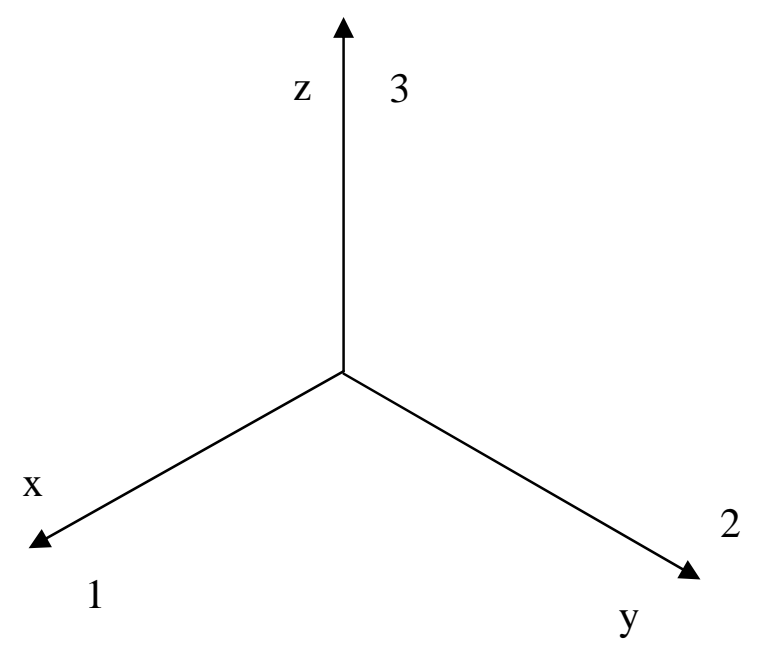

Figure 4.14: Basic 3 axis coordinate system

To summarize,

1-rotation $=$ Roll $=\phi$

2-rotation $=$ Pitch $=\theta$

3-rotation $=$ Yaw $=\psi$ 
For a vehicle traveling on a straight and level road executing a turn, equation 4.122 is used from above:

$3-1-2=\psi-\phi-\theta \quad$ Yaw - Roll - Pitch

Since the vehicle is entering a turn while driving on a straight and level road, it would seem that the first vehicle rotation would be yaw. Once the vehicle has begun to turn the second vehicle rotation would be roll, and then due to the combination of the other two, a pitch rotation might occur.

It should be noted that pitch is not included in the analysis. Although it is being including in the derivation, the pitch term will be neglected at the end.

These are the Euler rotation matrices for Yaw, Pitch, and Roll:

Yaw: $\left\{\begin{array}{l}x^{\prime} \\ y^{\prime} \\ z^{\prime}\end{array}\right\}=\left[\begin{array}{ccc}\cos \psi & \sin \psi & 0 \\ -\sin \psi & \cos \psi & 0 \\ 0 & 0 & 1\end{array}\right]\left\{\begin{array}{l}X \\ Y \\ Z\end{array}\right\}$

Roll: $\left\{\begin{array}{l}x \\ y \\ z\end{array}\right\}=\left[\begin{array}{ccc}1 & 0 & 0 \\ 0 & \cos \phi & \sin \phi \\ 0 & -\sin \phi & \cos \phi\end{array}\right]\left\{\begin{array}{l}x^{\prime \prime} \\ y^{\prime \prime} \\ z^{\prime \prime}\end{array}\right\}$

Pitch: $\left\{\begin{array}{l}x^{\prime \prime} \\ y^{\prime \prime} \\ z^{\prime \prime}\end{array}\right\}=\left[\begin{array}{ccc}\cos \theta & 0 & -\sin \theta \\ 0 & 1 & 0 \\ \sin \theta & 0 & \cos \theta\end{array}\right]\left\{\begin{array}{l}x^{\prime} \\ y^{\prime} \\ z^{\prime}\end{array}\right\}$

This is the determinant form of the cross product of angular velocity and yaw rate:

$$
\vec{\omega} \times \vec{r}=\left|\begin{array}{ccc}
i & j & k \\
\omega_{x} & \omega_{y} & \omega_{z} \\
x & y & z
\end{array}\right|
$$

To come up with the proper rotation matrix equation, the process begins with a rotation about the z-axis, Yaw. When working with Euler rotations it is important to keep in mind that the process essentially works "backwards". In other words, consider whichever Euler rotation is last in the chosen sequence, which in this case is Pitch (see equation 4.132, above). That becomes the rotation that the other two rotations are transformed to. In this case, these are Yaw and Roll.

Since the chosen Euler angle sequence here is Yaw - Roll - Pitch, the Yaw rotation first has to be transformed to Roll coordinates, and then that result is transformed to Pitch coordinates. This is accomplished by premultiplying the Yaw coordinates by the Roll Euler angle rotation matrix: 


$$
\left[\begin{array}{ccc}
1 & 0 & 0 \\
0 & \cos \phi & \sin \phi \\
0 & -\sin \phi & \cos \phi
\end{array}\right]\left[\begin{array}{c}
0 \\
0 \\
\dot{\psi}
\end{array}\right]
$$

This result is then pre-multiplied by the Pitch Euler angle rotation matrix:

$$
\left[\begin{array}{ccc}
\cos \theta & 0 & -\sin \theta \\
0 & 1 & 0 \\
\sin \theta & 0 & \cos \theta
\end{array}\right]\left[\begin{array}{ccc}
1 & 0 & 0 \\
0 & \cos \phi & \sin \phi \\
0 & -\sin \phi & \cos \phi
\end{array}\right]\left[\begin{array}{c}
0 \\
0 \\
\dot{\psi}
\end{array}\right]
$$

Similarly for the Roll Euler angle, the Roll coordinates are transformed to Pitch coordinates. The Roll coordinates are pre-multiplied by the Pitch Euler angle rotation matrix:

$$
\left[\begin{array}{ccc}
\cos \theta & 0 & -\sin \theta \\
0 & 1 & 0 \\
\sin \theta & 0 & \cos \theta
\end{array}\right]\left[\begin{array}{l}
\dot{\phi} \\
0 \\
0
\end{array}\right]
$$

Since Pitch is the final Euler angle in the chosen sequence, the Pitch coordinates don't need to be pre-multiplied by anything:

$$
\left[\begin{array}{c}
0 \\
\dot{\theta} \\
0
\end{array}\right]
$$

Summing the results of these equations (4.135 - 4.137), the following Euler transformation equation is obtained:

$$
\left[\begin{array}{c}
0 \\
\dot{\theta} \\
0
\end{array}\right]+\left[\begin{array}{ccc}
\cos \theta & 0 & -\sin \theta \\
0 & 1 & 0 \\
\sin \theta & 0 & \cos \theta
\end{array}\right]\left[\begin{array}{l}
\dot{\phi} \\
0 \\
0
\end{array}\right]+\left[\begin{array}{ccc}
\cos \theta & 0 & -\sin \theta \\
0 & 1 & 0 \\
\sin \theta & 0 & \cos \theta
\end{array}\right]\left[\begin{array}{ccc}
1 & 0 & 0 \\
0 & \cos \phi & \sin \phi \\
0 & -\sin \phi & \cos \phi
\end{array}\right]\left[\begin{array}{c}
0 \\
0 \\
\dot{\psi}
\end{array}\right]
$$

Carrying out the pre-multiplications for the Roll to Pitch transformation yields:

$$
\left[\begin{array}{ccc}
\cos \theta & 0 & -\sin \theta \\
0 & 1 & 0 \\
\sin \theta & 0 & \cos \theta
\end{array}\right]\left[\begin{array}{l}
\dot{\phi} \\
0 \\
0
\end{array}\right]=\left[\begin{array}{c}
(\cos \theta) \dot{\phi} \\
0 \\
(\sin \theta) \dot{\phi}
\end{array}\right]
$$


Likewise, carrying out the transformation from Yaw to Roll results in:

$$
\left[\begin{array}{ccc}
\cos \theta & 0 & -\sin \theta \\
0 & 1 & 0 \\
\sin \theta & 0 & \cos \theta
\end{array}\right]\left[\begin{array}{ccc}
1 & 0 & 0 \\
0 & \cos \phi & \sin \phi \\
0 & -\sin \phi & \cos \phi
\end{array}\right]\left[\begin{array}{l}
0 \\
0 \\
\dot{\psi}
\end{array}\right]=\left[\begin{array}{ccc}
\cos \theta & 0 & -\sin \theta \\
0 & 1 & 0 \\
\sin \theta & 0 & \cos \theta
\end{array}\right]\left[\begin{array}{c}
0 \\
(\sin \phi) \dot{\psi} \\
(\cos \phi) \dot{\psi}
\end{array}\right]
$$

Transforming this new Yaw-Roll matrix to Pitch results in:

$$
\left[\begin{array}{ccc}
\cos \theta & 0 & -\sin \theta \\
0 & 1 & 0 \\
\sin \theta & 0 & \cos \theta
\end{array}\right]\left[\begin{array}{c}
0 \\
(\sin \phi) \dot{\psi} \\
(\cos \phi) \dot{\psi}
\end{array}\right]=\left[\begin{array}{c}
(-\sin \theta)(\cos \phi) \dot{\psi} \\
(\sin \phi) \dot{\psi} \\
(\cos \theta)(\cos \phi) \dot{\psi}
\end{array}\right]
$$

Summing equations 4.137, 4.139, and 4.141 gives:

$$
\left[\begin{array}{c}
0 \\
\dot{\theta} \\
0
\end{array}\right]+\left[\begin{array}{c}
(\cos \theta) \dot{\phi} \\
0 \\
(\sin \theta) \dot{\phi}
\end{array}\right]+\left[\begin{array}{c}
(-\sin \theta)(\cos \phi) \dot{\psi} \\
(\sin \phi) \dot{\psi} \\
(\cos \theta)(\cos \phi) \dot{\psi}
\end{array}\right]
$$

This new equation contains the terms of the angular velocities in matrix form. Extracting the respective terms and writing them individually:

$$
\begin{aligned}
& \omega_{x}=(\cos \theta) \dot{\phi}-(\sin \theta)(\cos \phi) \dot{\psi} \\
& \omega_{y}=\dot{\theta}+(\sin \phi) \dot{\psi} \\
& \omega_{z}=(\sin \theta) \dot{\phi}+(\cos \theta)(\cos \phi) \dot{\psi}
\end{aligned}
$$

Now since the rotational kinetic energy is defined as $T=\frac{1}{2} I \omega^{2}$, each of the angular velocity terms in equations $4.143,4.144$, and 4.145 needs to be squared in order to write the full kinetic energy equation. Therefore squaring each of these angular velocities results in:

$$
\begin{aligned}
& \omega_{x}=(\cos \theta) \dot{\phi}-(\sin \theta)(\cos \phi) \dot{\psi} \\
& \omega_{x}^{2}=[(\cos \theta) \dot{\phi}-(\sin \theta)(\cos \phi) \dot{\psi}]^{2} \\
& \omega_{x}^{2}=[(\cos \theta) \dot{\phi}-(\sin \theta)(\cos \phi) \dot{\psi}][(\cos \theta) \dot{\phi}-(\sin \theta)(\cos \phi) \dot{\psi}] \\
& \omega_{x}^{2}=\dot{\phi}^{2} \cos ^{2} \theta-\dot{\phi} \dot{\psi}(\sin \theta)(\cos \theta)(\cos \phi)-\dot{\phi} \dot{\psi}(\cos \theta)(\sin \theta)(\cos \phi)+\dot{\psi}^{2}\left(\sin ^{2} \theta\right)\left(\cos ^{2} \phi\right) \\
& \omega_{x}^{2}=\dot{\phi}^{2} \cos ^{2} \theta-2 \dot{\phi} \dot{\psi}(\sin \theta)(\cos \theta)(\cos \phi)+\dot{\psi}^{2}\left(\sin ^{2} \theta\right)\left(\cos ^{2} \phi\right)
\end{aligned}
$$




$$
\begin{aligned}
\omega_{y} & =\dot{\theta}+(\sin \phi) \dot{\psi} \\
\omega_{y}^{2} & =[\dot{\theta}+(\sin \phi) \dot{\psi}]^{2} \\
\omega_{y}^{2} & =[\dot{\theta}+(\sin \phi) \dot{\psi}][\dot{\theta}+(\sin \phi) \dot{\psi}] \\
\omega_{y}^{2} & =\dot{\theta}^{2}+\dot{\theta} \dot{\psi} \sin \phi+\dot{\theta} \dot{\psi} \sin \phi+\dot{\psi}^{2} \sin ^{2} \phi \\
\omega_{y}^{2} & =\dot{\theta}^{2}+2 \dot{\theta} \dot{\psi} \sin \phi+\dot{\psi}^{2} \sin ^{2} \phi \\
\omega_{z} & =(\sin \theta) \dot{\phi}+(\cos \theta)(\cos \phi) \dot{\psi} \\
\omega_{z}^{2} & =[(\sin \theta) \dot{\phi}+(\cos \theta)(\cos \phi) \dot{\psi}]^{2} \\
\omega_{z}^{2} & =[(\sin \theta) \dot{\phi}+(\cos \theta)(\cos \phi) \dot{\psi}][(\sin \theta) \dot{\phi}+(\cos \theta)(\cos \phi) \dot{\psi}] \\
\omega_{z}^{2} & =\dot{\phi}^{2}\left(\sin { }^{2} \theta\right)+\dot{\phi} \dot{\psi}(\sin \theta)(\cos \theta)(\cos \phi)+\dot{\phi} \dot{\psi}(\sin \theta)(\cos \theta)(\cos \phi)+\dot{\psi}^{2}\left(\cos ^{2} \theta\right)\left(\cos ^{2} \phi\right) \\
\omega_{z}^{2} & =\dot{\phi}^{2} \sin ^{2} \theta+2 \dot{\phi} \dot{\psi}(\sin \theta)(\cos \theta)(\cos \phi)+\dot{\psi}^{2}\left(\cos ^{2} \theta\right)\left(\cos ^{2} \phi\right)
\end{aligned}
$$

Assuming $\theta$ and $\phi$ are small, $\begin{array}{r}\sin \theta \approx \theta \\ \cos \theta \approx 1\end{array}$ and $\begin{array}{r}\sin \phi \approx \phi \\ \cos \phi \approx 1\end{array}$

and since the yaw angle is $\psi$, the yaw rate is $\dot{\psi}=r$. Therefore equations 4.146, 4.147, and 4.148 can be rewritten as:

$$
\begin{aligned}
& \omega_{x}^{2}=\dot{\phi}^{2} \cos ^{2} \theta-2 \dot{\phi} \dot{\psi}(\sin \theta)(\cos \theta)(\cos \phi)+\dot{\psi}^{2}\left(\sin ^{2} \theta\right)\left(\cos ^{2} \phi\right) \\
& \omega_{x}^{2}=\dot{\phi}^{2}-2 \dot{\phi} \dot{\psi} \theta+\dot{\psi}^{2} \theta^{2} \\
& \omega_{x}^{2}=\dot{\phi}^{2}-2 \dot{\phi} \theta r+r^{2} \theta^{2} \\
& \omega_{y}^{2}=\dot{\theta}^{2}+2 \dot{\theta} \dot{\psi} \sin \phi+\dot{\psi}^{2} \sin ^{2} \phi \\
& \omega_{y}^{2}=\dot{\theta}^{2}+2 \dot{\theta} \dot{\psi} \phi+\dot{\psi}^{2} \phi^{2} \\
& \omega_{y}^{2}=\dot{\theta}^{2}+2 \dot{\theta} \phi r+r^{2} \phi^{2} \\
& \omega_{z}^{2}=\dot{\phi}^{2} \sin ^{2} \theta+2 \dot{\phi} \dot{\psi}(\sin \theta)(\cos \theta)(\cos \phi)+\dot{\psi}^{2}\left(\cos ^{2} \theta\right)\left(\cos ^{2} \phi\right) \\
& \omega_{z}^{2}=\dot{\phi}^{2} \theta^{2}+2 \dot{\phi} \dot{\psi} \theta+\dot{\psi}^{2} \\
& \omega_{z}^{2}=\dot{\phi}^{2} \theta^{2}+2 \dot{\phi} \theta r+r^{2}
\end{aligned}
$$


Summarizing the results of equations $4.149,4.150$, and 4.151 yields:

$$
\begin{aligned}
& \omega_{x}^{2}=\dot{\phi}^{2}-2 \dot{\phi} \theta r+r^{2} \theta^{2} \\
& \omega_{y}^{2}=\dot{\theta}^{2}+2 \dot{\theta} \phi r+r^{2} \phi^{2} \\
& \omega_{z}^{2}=\dot{\phi}^{2} \theta^{2}+2 \dot{\phi} \theta r+r^{2}
\end{aligned}
$$

The following figures are used to determine the translational terms for the kinetic energy:

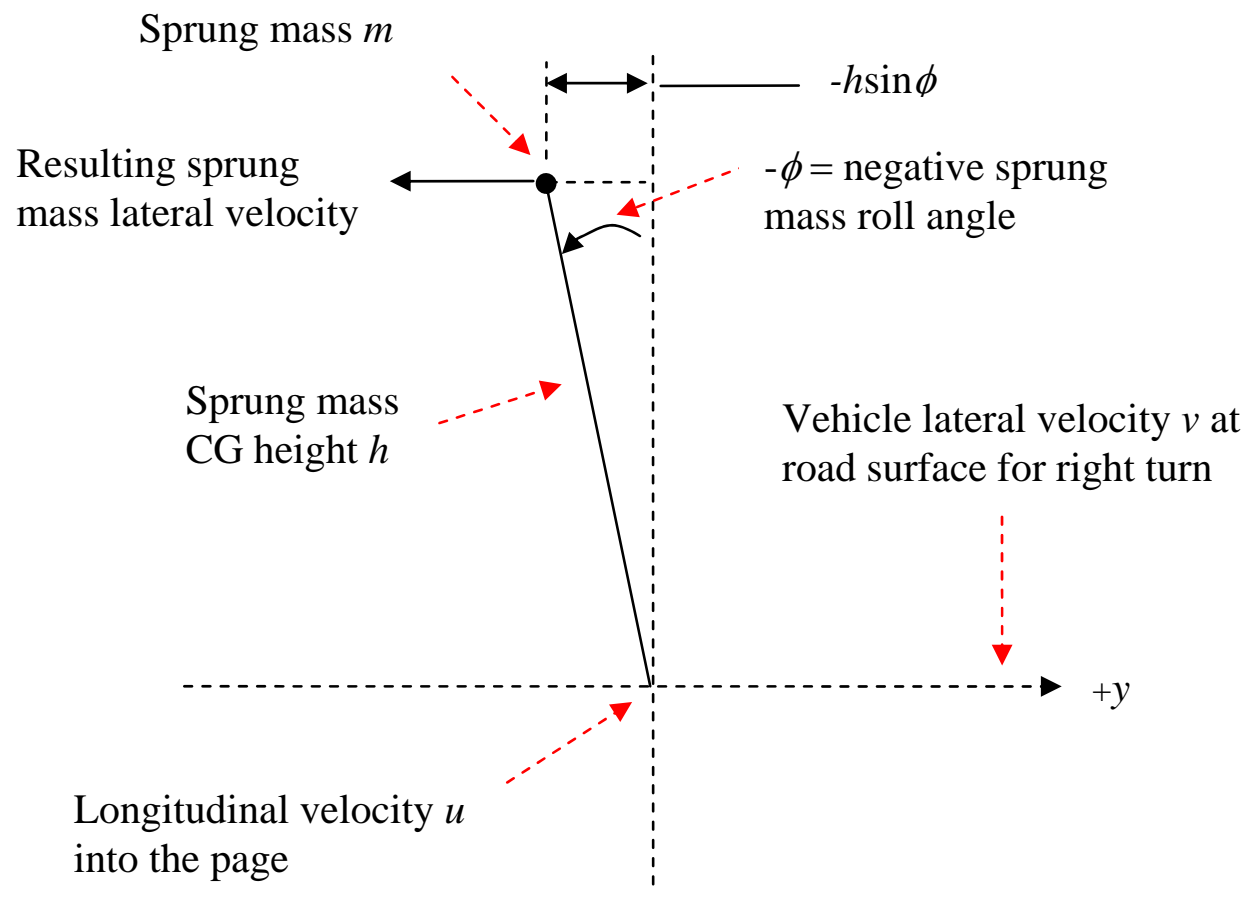

Figure 4.15 : Illustrating the lateral translational velocity term of the kinetic energy. View is from the rear of the vehicle, looking forward.

As the vehicle executes a right turn it will have a lateral velocity $v$ in the positive $y$ direction plus a rotational term due to the sprung mass $m$ rolling to the left. Based on Figures 4.2 and 4.13, rolling to the left, i.e. counterclockwise as viewed from the rear, is a negative roll angle. Since positive $y$ is to the right and the sprung mass is translating to the left, there is an additional negative sign in front of the rotational term. This rotational term is the angular velocity of the sprung mass, equal to the sprung mass height $h$ crossed with the sprung mass roll rate $\dot{\phi}$. The equation for this is:

$v-h(-\dot{\phi})=v+h \dot{\phi}$

To find the longitudinal translational velocity term Figures 4.15 and 4.16 (see below) can be used. Recall from Figure 4.15 that in a right turn, the sprung mass will translate a lateral distance equal to $(-h \sin \phi)$. Using the small angle assumption this can be rewritten as $(-h \phi)$. This distance 
can be defined as $l=(-h \phi)$. Considering a top view of a simplified vehicle in a right turn, Figure 4.16:

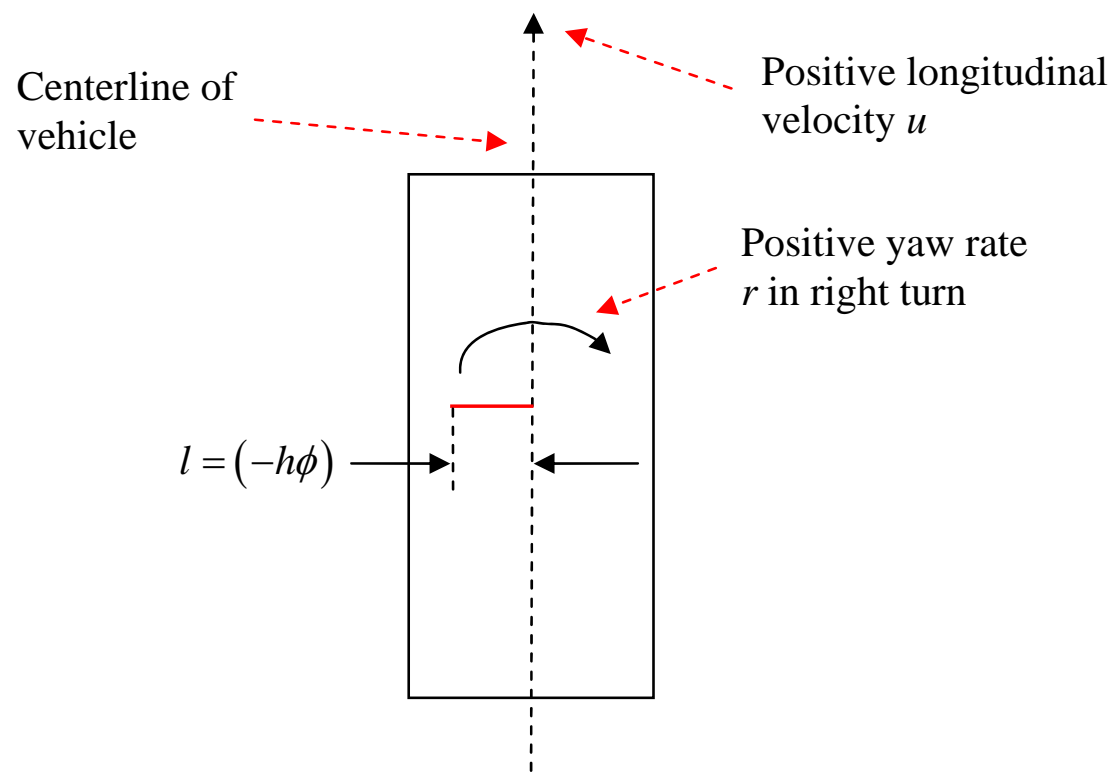

Figure 4.16 : Simplified top view of vehicle illustrating longitudinal translational velocity term of kinetic energy

In this instance, the total longitudinal translational velocity term of the kinetic energy is equal to:

$u+l r=u-h \phi r$

where the $l r$ term is the cross product of the angular velocity $r$ and the distance $l=(-h \phi)$.

Writing out the full kinetic energy term:

$$
\begin{aligned}
& T=\frac{1}{2} m\left\{(u-h \phi r)^{2}+(v+h \dot{\phi})^{2}\right\}+\frac{1}{2} I_{x}\left\{\dot{\phi}^{2}-2 \dot{\phi} \theta r+r^{2} \theta^{2}\right\}+\ldots \\
& \frac{1}{2} I_{y}\left\{\dot{\theta}^{2}+2 \dot{\theta} \phi r+r^{2} \phi^{2}\right\}+\frac{1}{2} I_{z}\left\{\theta^{2} \dot{\phi}^{2}+2 \dot{\phi} \theta r+r^{2}\right\}-I_{x z} r \dot{\phi}
\end{aligned}
$$

The final term: $-I_{x z} r \dot{\phi}$ contains the inertia cross product $I_{x z}$. This equation matches the kinetic energy equation shown in Pacejka [12].

Expanding the first translational kinetic energy term:

$$
\begin{aligned}
& (u-h \phi r)^{2}=(u-h \phi r)(u-h \phi r)=u^{2}-u h \phi r-u h \phi r+(h \phi r)^{2} \\
& (u-h \phi r)^{2}=u^{2}-2 u h \phi r+(h \phi r)^{2}
\end{aligned}
$$


Expanding the second translational kinetic energy term:

$$
\begin{aligned}
& (v+h \dot{\phi})^{2}=(v+h \dot{\phi})(v+h \dot{\phi})=v^{2}+v h \dot{\phi}+v h \dot{\phi}+(h \dot{\phi})^{2} \\
& (v+h \dot{\phi})^{2}=v^{2}+2 v h \dot{\phi}+(h \dot{\phi})^{2}
\end{aligned}
$$

The next step is to take the partial derivatives of the kinetic energy, beginning with $\frac{\partial T}{\partial u}$, the partial derivative of the kinetic energy with respect to the forward vehicle velocity $u$. Since the only term in equation 4.154, above, that contains a $u$ is the longitudinal translational kinetic energy term the partial derivative of the kinetic energy equation with respect to $u$ is:

$$
\frac{\partial T}{\partial u}=\frac{\partial}{\partial u}\left[\frac{1}{2} m\left(u^{2}-2 u h \phi r+(h \phi r)^{2}\right)\right]=\frac{1}{2} m(2 u-2 h \phi r)
$$

There is no $u$ in the third component, $(h \phi r)^{2}$, of equation 4.157 and thus the partial derivative of this term with respect to $u$ is zero. This leaves:

$\frac{\partial T}{\partial u}=m(u-h \phi r)$

Since the lateral vehicle velocity $v$ only appears in the lateral translational kinetic energy term the partial derivative of the kinetic energy equation with respect to $v$ is:

$$
\frac{\partial T}{\partial v}=\frac{\partial}{\partial v}\left[\frac{1}{2} m\left(v^{2}+2 v h \dot{\phi}+(h \dot{\phi})^{2}\right)\right]=\frac{1}{2} m(2 v+2 h \dot{\phi})
$$

There is no $v$ in the third component, $(h \dot{\phi})^{2}$, of equation 4.159 and thus the partial derivative of this term with respect to $v$ is zero. This leaves:

$$
\frac{\partial T}{\partial v}=m(v+h \dot{\phi})
$$

The next step is to take the partial derivative of the kinetic energy equation with respect to the roll angle $\phi$. Since the roll angle $\phi$ only appears in longitudinal translational kinetic energy term as well as the second rotational kinetic energy term, i.e. Pitch, the partial derivative of the kinetic energy equation with respect to $\phi$ is:

$$
\frac{\partial T}{\partial \phi}=\frac{\partial}{\partial \phi}\left[\frac{1}{2} m\left\{(u-h \phi r)^{2}\right\}+\frac{1}{2} I_{y}\left\{\dot{\theta}^{2}+2 \dot{\theta} \phi r+r^{2} \phi^{2}\right\}\right]
$$


Substituting in the expanded form for the translational term from equation 4.155:

$$
\frac{\partial T}{\partial \phi}=\frac{\partial}{\partial \phi}\left[\frac{1}{2} m\left\{u^{2}-2 u h \phi r+(h \phi r)^{2}\right\}+\frac{1}{2} I_{y}\left\{\dot{\theta}^{2}+2 \dot{\theta} \phi r+r^{2} \phi^{2}\right\}\right]
$$

There is no $\phi$ in either the first component of the translational term, $u^{2}$, or the first component of the Pitch rotational term, $\dot{\theta}^{2}$, and thus the partial derivatives of these two terms with respect to $\phi$ is zero. This leaves:

$$
\frac{\partial T}{\partial \phi}=\frac{\partial}{\partial \phi}\left[\frac{1}{2} m\left\{(h \phi r)^{2}-2 u h \phi r\right\}+\frac{1}{2} I_{y}\left\{2 \dot{\theta} \phi r+r^{2} \phi^{2}\right\}\right]
$$

Taking the partial derivative with respect to $\phi$ yields:

$\frac{\partial T}{\partial \phi}=\frac{1}{2} m\left\{2 \phi h^{2} r^{2}-2 u h r\right\}+\frac{1}{2} I_{y}\left\{2 \dot{\theta} r+2 \phi r^{2}\right\}$

This leaves:

$\frac{\partial T}{\partial \phi}=m\left\{\phi h^{2} r^{2}-u h r\right\}+I_{y}\left\{\dot{\theta} r+\phi r^{2}\right\}$

The next step is to take the partial derivative of the kinetic energy equation with respect to $\dot{\phi}$. Looking at the full kinetic energy equation 4.154, none of the following terms contain a $\dot{\phi}$ :

- The longitudinal translational kinetic energy term, $(u-h \phi r)^{2}$

- The third term of the Roll angular velocity, $r^{2} \theta^{2}$

- None of the terms associated with the Pitch

- The last term in the Yaw angular velocity, $r^{2}$

Therefore the partial derivatives of all of these terms with respect to $\dot{\phi}$ are zero. This leaves:

$$
\frac{\partial T}{\partial \dot{\phi}}=\frac{\partial}{\partial \dot{\phi}}\left[\frac{1}{2} m(v+h \dot{\phi})^{2}+\frac{1}{2} I_{x}\left\{\dot{\phi}^{2}-2 \dot{\phi} \theta r\right\}+\frac{1}{2} I_{z}\left\{\theta^{2} \dot{\phi}^{2}+2 \dot{\phi} \theta r\right\}-I_{x z} r \dot{\phi}\right]
$$

The next step is to substitute in the expanded form for the remaining translational term from equation 4.156, leaving:

$$
\frac{\partial T}{\partial \dot{\phi}}=\frac{\partial}{\partial \dot{\phi}}\left[\frac{1}{2} m\left\{v^{2}+2 v h \dot{\phi}+(h \dot{\phi})^{2}\right\}+\frac{1}{2} I_{x}\left\{\dot{\phi}^{2}-2 \dot{\phi} \theta r\right\}+\frac{1}{2} I_{z}\left\{\theta^{2} \dot{\phi}^{2}+2 \dot{\phi} \theta r\right\}-I_{x z} r \dot{\phi}\right]
$$


One more term can be eliminated before proceeding with taking the partial derivative. The first term in the expanded translational component is $v^{2}$. Since this does not contain a $\dot{\phi}$, its partial derivative with respect to $\dot{\phi}$ is zero. Thus:

$$
\frac{\partial T}{\partial \dot{\phi}}=\frac{\partial}{\partial \dot{\phi}}\left[\frac{1}{2} m\left\{2 v h \dot{\phi}+(h \dot{\phi})^{2}\right\}+\frac{1}{2} I_{x}\left\{\dot{\phi}^{2}-2 \dot{\phi} \theta r\right\}+\frac{1}{2} I_{z}\left\{\theta^{2} \dot{\phi}^{2}+2 \dot{\phi} \theta r\right\}-I_{x z} r \dot{\phi}\right]
$$

Taking the partial derivative:

$$
\frac{\partial T}{\partial \dot{\phi}}=\frac{1}{2} m\left\{2 v h+2 \dot{\phi} h^{2}\right\}+\frac{1}{2} I_{x}\{2 \dot{\phi}-2 \theta r\}+\frac{1}{2} I_{z}\left\{2 \dot{\phi} \theta^{2}+2 \theta r\right\}-I_{x z} r
$$

This leaves:

$$
\frac{\partial T}{\partial \dot{\phi}}=m\left\{v h+\dot{\phi} h^{2}\right\}+I_{x}\{\dot{\phi}-\theta r\}+I_{z}\left\{\dot{\phi} \theta^{2}+\theta r\right\}-I_{x z} r
$$

The last partial derivative that needs to be taken of the kinetic energy equation will be with respect to the yaw rate, $r$. By inspection of the full kinetic energy equation 4.154, it can be seen that the following terms do not contain the yaw rate term $r$ :

- The lateral translational kinetic energy term, $(v+h \dot{\phi})^{2}$

- The first component of the Roll angular velocity, $\dot{\phi}^{2}$

- The first component of the Pitch angular velocity, $\dot{\theta}^{2}$

- The first component of the Yaw angular velocity, $\theta^{2} \dot{\phi}^{2}$

As such, their partial derivatives with respect to $r$ will be zero.

$$
\frac{\partial T}{\partial r}=\frac{\partial}{\partial r}\left[\frac{1}{2} m(u-h \phi r)^{2}+\frac{1}{2} I_{x}\left\{r^{2} \theta^{2}-2 \dot{\phi} \theta r\right\}+\frac{1}{2} I_{y}\left\{2 \dot{\theta} \phi r+r^{2} \phi^{2}\right\}+\frac{1}{2} I_{z}\left\{2 \dot{\phi} \theta r+r^{2}\right\}-I_{x z} r \dot{\phi}\right]
$$

Substituting in the expanded form for the longitudinal translational kinetic energy we have:

$$
\frac{\partial T}{\partial r}=\frac{\partial}{\partial r}\left[\begin{array}{l}
\left.\frac{1}{2} m\left\{u^{2}-2 u h \phi r+(h \phi r)^{2}\right\}+\frac{1}{2} I_{x}\left\{r^{2} \theta^{2}-2 \dot{\phi} \theta r\right\}+\frac{1}{2} I_{y}\left\{2 \dot{\theta} \phi r+r^{2} \phi^{2}\right\}+\ldots\right] \\
\frac{1}{2} I_{z}\left\{2 \dot{\phi} \theta r+r^{2}\right\}-I_{x z} r \dot{\phi}
\end{array}\right]
$$


The $u^{2}$ term can be eliminated from the translational component since it does not contain an $r$ :

$$
\frac{\partial T}{\partial r}=\frac{\partial}{\partial r}\left[\begin{array}{l}
\left.\frac{1}{2} m\left\{(h \phi r)^{2}-2 u h \phi r\right\}+\frac{1}{2} I_{x}\left\{r^{2} \theta^{2}-2 \dot{\phi} \theta r\right\}+\frac{1}{2} I_{y}\left\{2 \dot{\theta} \phi r+r^{2} \phi^{2}\right\}+\ldots\right] \\
\frac{1}{2} I_{z}\left\{2 \dot{\phi} \theta r+r^{2}\right\}-I_{x z} r \dot{\phi}
\end{array}\right]
$$

Taking the partial derivative with respect to $r$ :

$$
\frac{\partial T}{\partial r}=\frac{1}{2} m\left\{2 r h^{2} \phi^{2}-2 u h \phi\right\}+\frac{1}{2} I_{x}\left\{2 r \theta^{2}-2 \dot{\phi} \theta\right\}+\frac{1}{2} I_{y}\left\{2 \dot{\theta} \phi+2 r \phi^{2}\right\}+\frac{1}{2} I_{z}\{2 \dot{\phi} \theta+2 r\}-I_{x z} \dot{\phi}
$$

This leaves:

$$
\frac{\partial T}{\partial r}=m\left\{r h^{2} \phi^{2}-u h \phi\right\}+I_{x}\left\{r \theta^{2}-\dot{\phi} \theta\right\}+I_{y}\left\{\dot{\theta} \phi+r \phi^{2}\right\}+I_{z}\{\dot{\phi} \theta+r\}-I_{x z} \dot{\phi}
$$

The next step is to take the time derivatives of $\frac{\partial T}{\partial u}$ and $\frac{\partial T}{\partial v}$, starting with $\frac{\partial T}{\partial u}$ :

$$
\frac{d}{d t} \frac{\partial T}{\partial u}=\frac{d}{d t}[m(u-h \phi r)]
$$

Consideration has to be given as to which terms are time dependent and which are not. There are two terms that are not time dependent: vehicle mass $m$ and CG height $h$. The remaining three terms: forward vehicle velocity $u$, roll angle $\phi$, and yaw rate $r$ are time dependent.

To assist in carrying out the time derivative of equation 4.176, specifically the $h \phi r$ component, the product rule can be used which has the form:

$$
(f g)^{\prime}=f^{\prime} g+f g^{\prime}
$$

where $f$ and $g$ are two variables, represented here by $\phi$ and $r$.

Carrying out the time derivative of equation 4.176 results in:

$$
\frac{d}{d t} \frac{\partial T}{\partial u}=m[\dot{u}-(h \dot{\phi} r+h \phi \dot{r})]=m(\dot{u}-h \dot{\phi} r-h \phi \dot{r})
$$


Doing the same thing for equation 4.160:

$$
\begin{aligned}
& \frac{\partial T}{\partial v}=m(v+h \dot{\phi}) \\
& \frac{d}{d t} \frac{\partial T}{\partial v}=\frac{d}{d t}[m(v+h \dot{\phi})]=m(\dot{v}+h \ddot{\phi})
\end{aligned}
$$

The next step in the derivation process is to multiply several of the partial derivatives by the yaw rate $r$ as well as the lateral and longitudinal velocities $v$ and $u$, respectively.

$$
\begin{aligned}
& r \frac{\partial T}{\partial u}=r[m(u-h \phi r)]=r m(u-h \phi r)=m r u-m h \phi r^{2} \\
& r \frac{\partial T}{\partial v}=r[m(v+h \dot{\phi})]=m r(v+h \dot{\phi})=m r v+m r h \dot{\phi} \\
& v \frac{\partial T}{\partial u}=v[m(u-h \phi r)]=v m(u-h \phi r)=m v u-m v h \phi r \\
& u \frac{\partial T}{\partial v}=u[m(v+h \dot{\phi})]=m u(v+h \dot{\phi})=m v u+m u h \dot{\phi}
\end{aligned}
$$

The equations will now be written include virtual work. This will result in four new Lagrangian equations.

\section{Lagrange Equation 1}

Beginning with equation 4.90:

$$
\frac{d}{d t} \frac{\partial T}{\partial u}-r \frac{\partial T}{\partial v}=Q_{u}
$$

Substituting in the respective terms from equations 4.178 and 4.181 results in:

$$
m(\dot{u}-h \dot{\phi} r-h \phi \dot{r})-m r v-m r h \dot{\phi}=F_{x F} \cos \delta_{f}-F_{y F} \sin \delta_{f}+F_{x R}
$$

\section{Lagrange Equation 2}

Beginning with equation 4.91:

$$
\frac{d}{d t} \frac{\partial T}{\partial v}+r \frac{\partial T}{\partial u}=Q_{v}
$$


Substituting in the respective terms from equations 4.179 and 4.180 yields:

$$
m(\dot{v}+h \ddot{\phi})+m r u-m h \phi r^{2}=F_{x F} \sin \delta_{f}+F_{y_{F}} \cos \delta_{f}+F_{y R}
$$

\section{Lagrange Equation 3}

Beginning with equation 4.92:

$\frac{d}{d t} \frac{\partial T}{\partial r}-v \frac{\partial T}{\partial u}+u \frac{\partial T}{\partial v}=Q_{\psi}$

Recall from equation 4.175 that:

$\frac{\partial T}{\partial r}=m\left\{r h^{2} \phi^{2}-u h \phi\right\}+I_{x}\left\{r \theta^{2}-\dot{\phi} \theta\right\}+I_{y}\left\{\dot{\theta} \phi+r \phi^{2}\right\}+I_{z}\{\dot{\phi} \theta+r\}-I_{x z} \dot{\phi}$

Since the time derivative needs to be taken of this equation and there are multiple parts, this is best done by treating each part like a separate equation, taking the time derivative of each part, and then summing the results.

Part 1: This can be simplified slightly by factoring an $h$ out:

$\frac{d}{d t} \frac{\partial T}{\partial r}(1)=\frac{d}{d t}\left[m\left\{r h^{2} \phi^{2}-u h \phi\right\}\right]=\frac{d}{d t}\left[m h\left\{r h \phi^{2}-u \phi\right\}\right]$

Completing the time derivative results in:

$$
\begin{aligned}
& \frac{d}{d t} \frac{\partial T}{\partial r}(1)=\frac{d}{d t}\left[m h\left\{r h \phi^{2}-u \phi\right\}\right]=m h\left[h\left(2 \phi \dot{\phi} r+\dot{r} \phi^{2}\right)-(\dot{u} \phi+u \dot{\phi})\right] \\
& \frac{d}{d t} \frac{\partial T}{\partial r}(1)=m h\left[h\left(2 \phi \dot{\phi} r+\dot{r} \phi^{2}\right)-\dot{u} \phi-u \dot{\phi}\right]
\end{aligned}
$$

Part 2:

$$
\begin{aligned}
& \frac{d}{d t} \frac{\partial T}{\partial r}(2)=\frac{d}{d t}\left[I_{x}\left\{r \theta^{2}-\dot{\phi} \theta\right\}\right]=I_{x}\left[\left(2 \theta \dot{\theta} r+\dot{r} \theta^{2}\right)-(\ddot{\phi} \theta+\dot{\phi} \dot{\theta})\right] \\
& \frac{d}{d t} \frac{\partial T}{\partial r}(2)=I_{x}\left[\left(2 \theta \dot{\theta} r+\dot{r} \theta^{2}\right)-\ddot{\phi} \theta-\dot{\phi} \dot{\theta}\right]
\end{aligned}
$$


Part 3:

$\frac{d}{d t} \frac{\partial T}{\partial r}(3)=\frac{d}{d t}\left[I_{y}\left\{\dot{\theta} \phi+r \phi^{2}\right\}\right]=I_{y}\left[(\ddot{\theta} \phi+\dot{\theta} \dot{\phi})+\left(2 \phi \dot{\phi} r+\dot{r} \phi^{2}\right)\right]$

Part 4:

$\frac{d}{d t} \frac{\partial T}{\partial r}(4)=\frac{d}{d t}\left[I_{z}\{\dot{\phi} \theta+r\}\right]=I_{z}[\ddot{\phi} \theta+\dot{\phi} \dot{\theta}+\dot{r}]$

Part 5:

$\frac{d}{d t} \frac{\partial T}{\partial r}(5)=\frac{d}{d t}\left[-I_{x z} \dot{\phi}\right]=-I_{x z} \ddot{\phi}$

Putting these all back together results in:

$$
\begin{aligned}
& \frac{d}{d t} \frac{\partial T}{\partial r}=\frac{d}{d t} \frac{\partial T}{\partial r}(1)+\frac{d}{d t} \frac{\partial T}{\partial r}(2)+\frac{d}{d t} \frac{\partial T}{\partial r}(3)+\frac{d}{d t} \frac{\partial T}{\partial r}(4)+\frac{d}{d t} \frac{\partial T}{\partial r}(5) \\
& \frac{d}{d t} \frac{\partial T}{\partial r}=m h\left[h\left(2 \phi \dot{\phi} r+\dot{r} \phi^{2}\right)-\dot{u} \phi-u \dot{\phi}\right]+I_{x}\left[\left(2 \theta \dot{\theta} r+\dot{r} \theta^{2}\right)-\ddot{\phi} \theta-\dot{\phi} \dot{\theta}\right]+\ldots \\
& I_{y}\left[(\ddot{\theta} \phi+\dot{\theta} \dot{\phi})+\left(2 \phi \dot{\phi} r+\dot{r} \phi^{2}\right)\right]+I_{z}[\ddot{\phi} \theta+\dot{\phi} \dot{\theta}+\dot{r}]-I_{x z} \ddot{\phi}
\end{aligned}
$$

Now that this has been derived, it can be combined with the remainder of the terms in equations 4.182 and 4.183 to obtain:

$$
\begin{aligned}
& \frac{d}{d t} \frac{\partial T}{\partial r}-v \frac{\partial T}{\partial u}+u \frac{\partial T}{\partial v}=Q_{\psi} \\
& m h\left[h\left(2 \phi \dot{\phi} r+\dot{r} \phi^{2}\right)-\dot{u} \phi-u \dot{\phi}\right]+I_{x}\left[\left(2 \theta \dot{\theta} r+\dot{r} \theta^{2}\right)-\ddot{\phi} \theta-\dot{\phi} \dot{\theta}\right]+I_{y}\left[(\ddot{\theta} \phi+\dot{\theta} \dot{\phi})+\left(2 \phi \dot{\phi} r+\dot{r} \phi^{2}\right)\right]+\ldots \\
& I_{z}[\ddot{\phi} \theta+\dot{\phi} \dot{\theta}+\dot{r}]-I_{x z} \ddot{\phi}-[m v u-m v h \phi r]+[m v u+m u h \dot{\phi}]=Q_{\psi}
\end{aligned}
$$

The next step is to distribute the leading terms and eliminate the brackets and parenthesis so as to locate and group the terms that share common variables, i.e. $\dot{r}, \phi$, etc. more easily: 
$2 \phi \dot{\phi} r m h^{2}+\dot{r} \phi^{2} m h^{2}-m \dot{u} h \phi-m u h \dot{\phi}+2 I_{x} \theta \dot{\theta} r+I_{x} \dot{r} \theta^{2}-I_{x} \ddot{\phi} \theta-I_{x} \dot{\phi} \dot{\theta}+I_{y} \ddot{\theta} \phi+I_{y} \dot{\theta} \dot{\phi}+2 I_{y} \phi \dot{\phi} r+I_{y} \dot{r} \phi^{2}+\ldots$

$I_{z} \ddot{\phi} \theta+I_{z} \dot{\phi} \dot{\theta}+I_{z} \dot{r}-I_{x z} \ddot{\phi}-m v u+m v h \phi r+m v u+m u h \dot{\phi}=Q_{\psi}$

The following terms can be cancelled due to the fact that they contain the same variables but have opposite signs:

mvu

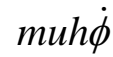

This leaves:

$2 \phi \dot{\phi} r m h^{2}+\dot{r} \phi^{2} m h^{2}-m \dot{u} h \phi+2 I_{x} \theta \dot{\theta} r+I_{x} \dot{r} \theta^{2}-I_{x} \ddot{\phi} \theta-I_{x} \dot{\phi} \dot{\theta}+I_{y} \ddot{\theta} \phi+I_{y} \dot{\theta} \dot{\phi}+2 I_{y} \phi \dot{\phi} r+I_{y} \dot{r} \phi^{2}+\ldots$

$I_{z} \ddot{\phi} \theta+I_{z} \dot{\phi} \dot{\theta}+I_{z} \dot{r}-I_{x z} \ddot{\phi}+m v h \phi r=Q_{\psi}$

The next step is to group the terms together that share common variables, i.e. $\dot{r}, \phi$, etc.

$\left[m h^{2} \phi^{2}+I_{x} \theta^{2}+I_{y} \phi^{2}+I_{z}\right] \dot{r}+\left[I_{z} \theta-I_{x z}-I_{x} \theta\right] \ddot{\phi}+m h \phi[-\dot{u}+v r]+\left[I_{y}+m h^{2}\right] 2 \phi \dot{\phi} r+\ldots$

$2 I_{x} \theta \dot{\theta} r+\left[I_{z}-I_{x}+I_{y}\right] \dot{\phi} \dot{\theta}+I_{y} \ddot{\theta} \phi=Q_{\psi}$

\section{Lagrange Equation 4}

Beginning with equation 4.93:

$\frac{d}{d t} \frac{\partial T}{\partial \dot{\phi}}-\frac{\partial T}{\partial \phi}+\frac{\partial U}{\partial \phi}=Q_{\phi}$

For the partial derivative of the kinetic energy equation with respect to $\dot{\phi}$ :

$\frac{\partial T}{\partial \dot{\phi}}=\frac{\partial}{\partial \dot{\phi}}\left[\begin{array}{l}\frac{1}{2} m\left\{(u-h \phi r)^{2}+(v+h \dot{\phi})^{2}\right\}+\frac{1}{2} I_{x}\left\{\dot{\phi}^{2}-2 \dot{\phi} \theta r+r^{2} \theta^{2}\right\}+\ldots \\ \frac{1}{2} I_{y}\left\{\dot{\theta}^{2}+2 \dot{\theta} \phi r+r^{2} \phi^{2}\right\}+\frac{1}{2} I_{z}\left\{\theta^{2} \dot{\phi}^{2}+2 \dot{\phi} \theta r+r^{2}\right\}-I_{x z} r \dot{\phi}\end{array}\right]$

Since the following terms do not contain a $\dot{\phi}$, their partial derivative with respect to $\dot{\phi}$ will be zero and can therefore be eliminated:

- The entire longitudinal translational velocity term $(u-h \phi r)^{2}$ 
- The third component of the Roll angular velocity $r^{2} \theta^{2}$

- All of the Pitch angular velocity $\frac{1}{2} I_{y}\left\{\dot{\theta}^{2}+2 \dot{\theta} \phi r+r^{2} \phi^{2}\right\}$

- The third component of the Yaw angular velocity $r^{2}$

Eliminating these terms results in a simplified version of equation 4.198:

$$
\frac{\partial T}{\partial \dot{\phi}}=\frac{\partial}{\partial \dot{\phi}}\left[\frac{1}{2} m(v+h \dot{\phi})^{2}+\frac{1}{2} I_{x}\left\{\dot{\phi}^{2}-2 \dot{\phi} \theta r\right\}+\frac{1}{2} I_{z}\left\{\theta^{2} \dot{\phi}^{2}+2 \dot{\phi} \theta r\right\}-I_{x z} r \dot{\phi}\right]
$$

Substituting in the expanded lateral translational velocity term:

$$
\frac{\partial T}{\partial \dot{\phi}}=\frac{\partial}{\partial \dot{\phi}}\left[\frac{1}{2} m\left\{v^{2}+2 v h \dot{\phi}+(h \dot{\phi})^{2}\right\}+\frac{1}{2} I_{x}\left\{\dot{\phi}^{2}-2 \dot{\phi} \theta r\right\}+\frac{1}{2} I_{z}\left\{\theta^{2} \dot{\phi}^{2}+2 \dot{\phi} \theta r\right\}-I_{x z} r \dot{\phi}\right]
$$

It can be seen that $v^{2}$ term can be cancelled from the translational component, resulting in:

$$
\frac{\partial T}{\partial \dot{\phi}}=\frac{\partial}{\partial \dot{\phi}}\left[\frac{1}{2} m\left\{2 v h \dot{\phi}+(h \dot{\phi})^{2}\right\}+\frac{1}{2} I_{x}\left\{\dot{\phi}^{2}-2 \dot{\phi} \theta r\right\}+\frac{1}{2} I_{z}\left\{\theta^{2} \dot{\phi}^{2}+2 \dot{\phi} \theta r\right\}-I_{x z} r \dot{\phi}\right]
$$

Taking the partial derivative yields:

$\frac{\partial T}{\partial \dot{\phi}}=\frac{1}{2} m\left\{2 v h+2 \dot{\phi} h^{2}\right\}+\frac{1}{2} I_{x}\{2 \dot{\phi}-2 \theta r\}+\frac{1}{2} I_{z}\left\{2 \dot{\phi} \theta^{2}+2 \theta r\right\}-I_{x z} r$

This leaves:

$\frac{\partial T}{\partial \dot{\phi}}=m\left\{v h+\dot{\phi} h^{2}\right\}+I_{x}\{\dot{\phi}-\theta r\}+I_{z}\left\{\dot{\phi} \theta^{2}+\theta r\right\}-I_{x z} r$

Taking the time derivative of equation 4.203:

$$
\begin{aligned}
& \frac{d}{d t} \frac{\partial T}{\partial \dot{\phi}}=\frac{d}{d t}\left[m\left\{v h+\dot{\phi} h^{2}\right\}+I_{x}\{\dot{\phi}-\theta r\}+I_{z}\left\{\dot{\phi} \theta^{2}+\theta r\right\}-I_{x z} r\right] \\
& \frac{d}{d t} \frac{\partial T}{\partial \dot{\phi}}=m\left\{\dot{v} h+\ddot{\phi} h^{2}\right\}+I_{x}\{\ddot{\phi}-\dot{\theta} r-\theta \dot{r}\}+I_{z}\left\{\ddot{\phi} \theta^{2}+2 \dot{\phi} \theta \dot{\theta}+\dot{\theta} r+\theta \dot{r}\right\}-I_{x z} \dot{r}
\end{aligned}
$$


Combining equations 4.99 and 4.103:

$$
\frac{\partial U}{\partial \phi}=k_{\phi} \phi-m g h \sin \phi
$$

and substituting equations 4.165, and 4.205, and 4.206 into equation 4.93 results in:

$$
\begin{aligned}
& \frac{d}{d t} \frac{\partial T}{\partial \dot{\phi}}-\frac{\partial T}{\partial \phi}+\frac{\partial U}{\partial \phi}=Q_{\phi} \\
& m\left\{\dot{v} h+\ddot{\phi} h^{2}\right\}+I_{x}\{\ddot{\phi}-\dot{\theta} r-\theta \dot{r}\}+\ldots \\
& I_{z}\left\{\ddot{\phi} \theta^{2}+2 \dot{\phi} \theta \dot{\theta}+\dot{\theta} r+\theta \dot{r}\right\}-I_{x z} \dot{r}-\left[m\left\{\phi h^{2} r^{2}-u h r\right\}+I_{y}\left\{\dot{\theta} r+\phi r^{2}\right\}\right]+\ldots \\
& k_{\phi} \phi-m g h \phi=Q_{\phi}
\end{aligned}
$$

Note that the small angle assumption was used to simplify equation 4.206.

Distributing the leading variables:

$$
\begin{aligned}
& m \dot{v} h+m \ddot{\phi} h^{2}+I_{x} \ddot{\phi}-I_{x} \dot{\theta} r-I_{x} \theta \dot{r}+\ldots \\
& I_{z} \ddot{\phi} \theta^{2}+2 I_{z} \dot{\phi} \theta \dot{\theta}+I_{z} \dot{\theta} r+I_{z} \theta \dot{r}-I_{x z} \dot{r}-m \phi h^{2} r^{2}+m u h r-I_{y} \dot{\theta} r-I_{y} \phi r^{2}+\ldots \\
& k_{\phi} \phi-m g h \phi=Q_{\phi}
\end{aligned}
$$

The terms can be grouped in the following manner:

$$
\begin{aligned}
& {\left[I_{x}+m h^{2}+I_{z} \theta^{2}\right] \ddot{\phi}+m h[\dot{v}+u r]+\left[-I_{x} \theta+I_{z} \theta-I_{x z}\right] \dot{r}+\ldots} \\
& {\left[-I_{y}-m h^{2}\right] \phi r^{2}+\left[-I_{x}+I_{z}-I_{y}\right] \dot{\theta} r+\ldots} \\
& 2 I_{z} \dot{\phi} \theta \dot{\theta}+\left[k_{\phi}-m g h\right] \phi=Q_{\phi}
\end{aligned}
$$

To summarize all of the work so far it will be helpful to display all four of the Lagrange equations in one location:

Equation 1 (4.184)

$$
m(\dot{u}-h \dot{\phi} r-h \phi \dot{r})-m r v-m r h \dot{\phi}=Q_{u}
$$

Lagrange Equation 1 
Equation 2 (4.185)

$m(\dot{v}+h \ddot{\phi})+m r u-m h \phi r^{2}=Q_{v}$

Lagrange Equation 2

Equation 3 (4.197)

$\left[m h^{2} \phi^{2}+I_{x} \theta^{2}+I_{y} \phi^{2}+I_{z}\right] \dot{r}+\left[I_{z} \theta-I_{x z}-I_{x} \theta\right] \ddot{\phi}+\ldots$

$m h \phi[-\dot{u}+v r]+\left[I_{y}+m h^{2}\right] 2 \phi \dot{\phi} r+\ldots$

Lagrange Equation 3

$2 I_{x} \theta \dot{\theta} r+\left[I_{z}-I_{x}+I_{y}\right] \dot{\phi} \dot{\theta}+I_{y} \ddot{\theta} \phi=Q_{\psi}$

Equation 4 (4.209)

$\left[I_{x}+m h^{2}+I_{z} \theta^{2}\right] \ddot{\phi}+m h[\dot{v}+u r]+\left[-I_{x} \theta+I_{z} \theta-I_{x z}\right] \dot{r}+\ldots$

$\left[-I_{y}-m h^{2}\right] \phi r^{2}+\left[-I_{x}+I_{z}-I_{y}\right] \dot{\theta} r+\ldots$

Lagrange Equation 4

$2 I_{z} \dot{\phi} \theta \dot{\theta}+\left[k_{\phi}-m g h\right] \phi=Q_{\phi}$

The next step is to substitute in the equations for the Qs .

The four new Lagrange equations will be steadily simplified until they can be put into matrix form. Since they will be referred to as Lagrange Equation 1, Lagrange Equation 2, etc, the numerical equation numbering convention will be suspended until the matrix is reached. Thus with each change made to these new Lagrange equations they can simply be referred to as Lagrange Set 1, Lagrange Set 2, etc.

Since there is a mass term $m$ present in each term on the left hand side in both the first and second Lagrange equations, they can be rewritten as:

\section{Lagrange Set 1}

$$
\begin{array}{ll}
m[\dot{u}-r v-h \phi \dot{r}-2 h r \dot{\phi}]=F_{x F} \cos \delta_{f}-F_{y F} \sin \delta_{f}+F_{x_{R}} & \text { Lagrange Equation } 1 \\
m\left[\dot{v}+r u+h \ddot{\phi}-h \phi r^{2}\right]=F_{x F} \sin \delta_{f}+F_{y F} \cos \delta_{f}+F_{y R} & \text { Lagrange Equation 2 }
\end{array}
$$




$$
\begin{aligned}
& {\left[m h^{2} \phi^{2}+I_{x} \theta^{2}+I_{y} \phi^{2}+I_{z}\right] \dot{r}+\left[I_{z} \theta-I_{x z}-I_{x} \theta\right] \ddot{\phi}+\ldots} \\
& m h \phi[-\dot{u}+v r]+\left[I_{y}+m h^{2}\right] 2 \phi \dot{\phi} r+\ldots \\
& 2 I_{x} \theta \dot{\theta} r+\left[I_{z}-I_{x}+I_{y}\right] \dot{\phi} \dot{\theta}+I_{y} \ddot{\theta} \phi=a F_{x F} \sin \delta_{f}+a F_{y F} \cos \delta_{f}+M_{z F}-b F_{y R}+M_{z R}
\end{aligned}
$$

Lagrange Equation 3

$\left[I_{x}+m h^{2}+I_{z} \theta^{2}\right] \ddot{\phi}+m h[\dot{v}+u r]+\left[-I_{x} \theta+I_{z} \theta-I_{x z}\right] \dot{r}+\ldots$

$\left[-I_{y}-m h^{2}\right] \phi r^{2}+\left[-I_{x}+I_{z}-I_{y}\right] \dot{\theta} r+\ldots$

Lagrange Equation 4

$2 I_{z} \dot{\phi} \theta \dot{\theta}+\left[k_{\phi}-m g h\right] \phi=-C_{\phi} \dot{\phi}$

\section{Lagrange Set 2}

The next step is to make additional simplifying assumptions and term cancellations. This process begins with the small steer angle assumption. It should be noted that the tire steer angle $\delta_{f}$ is being used, not the steering wheel angle (inside of the vehicle). Also, a small tire steer angle is defined as less than five degrees. See equation 4.1.

If a small tire steer angle is assumed, $\begin{aligned} & \sin \delta_{f} \sim \delta_{f} \\ & \cos \delta_{f} \sim 1\end{aligned}$ the four Lagrange equations become:

$$
\begin{array}{lr}
m[\dot{u}-r v-h \phi \dot{r}-2 h r \dot{\phi}]=F_{x F}-F_{y F} \delta_{f}+F_{x R} & \text { Lagrange Equation } 1 \\
m\left[\dot{v}+r u+h \ddot{\phi}-h \phi r^{2}\right]=F_{x_{F}} \delta_{f}+F_{y F}+F_{y_{R}} & \text { Lagrange Equation } 2 \\
{\left[m h^{2} \phi^{2}+I_{x} \theta^{2}+I_{y} \phi^{2}+I_{z}\right] \dot{r}+\left[I_{z} \theta-I_{x z}-I_{x} \theta\right] \ddot{\phi}+\ldots} & \\
m h \phi[-\dot{u}+v r]+\left[I_{y}+m h^{2}\right] 2 \phi \dot{\phi} r+\ldots & \\
2 I_{x} \theta \dot{\theta} r+\left[I_{z}-I_{x}+I_{y}\right] \dot{\phi} \dot{\theta}+I_{y} \ddot{\theta} \phi=a F_{x F} \delta_{f}+a F_{y F}+M_{z F}-b F_{y R}+M_{z R} &
\end{array}
$$$$
\left[I_{x}+m h^{2}+I_{z} \theta^{2}\right] \ddot{\phi}+m h[\dot{v}+u r]+\left[-I_{x} \theta+I_{z} \theta-I_{x z}\right] \dot{r}+\ldots
$$$$
\left[-I_{y}-m h^{2}\right] \phi r^{2}+\left[-I_{x}+I_{z}-I_{y}\right] \dot{\theta} r+\ldots
$$$$
2 I_{z} \dot{\phi} \dot{\theta}+C_{\phi} \dot{\phi}+\left[k_{\phi}-m g h\right] \phi=0
$$ 


\section{Lagrange Set 3}

The next assumption is that of the linear tire, which means equation 4.2 will be utilized. It is repeated here for the reader's convenience. A constant forward velocity can also be assumed, so $\dot{u}=0$.

$F_{y}=C_{\alpha} \alpha$

$m[-r v-h \phi \dot{r}-2 h r \dot{\phi}]=F_{x F}+F_{x R}-\delta_{f} C_{\alpha F} \alpha_{F}$

Lagrange Equation 1

$m\left[\dot{v}+r u+h \ddot{\phi}-h \phi r^{2}\right]=F_{x F} \delta_{f}+C_{\alpha F} \alpha_{f}+C_{\alpha R} \alpha_{R}$

Lagrange Equation 2

$\left[m h^{2} \phi^{2}+I_{x} \theta^{2}+I_{y} \phi^{2}+I_{z}\right] \dot{r}+\left[I_{z} \theta-I_{x z}-I_{x} \theta\right] \ddot{\phi}+\ldots$

$m h \phi[v r]+\left[I_{y}+m h^{2}\right] 2 \phi \dot{\phi} r+\ldots$

$2 I_{x} \theta \dot{\theta} r+\left[I_{z}-I_{x}+I_{y}\right] \dot{\phi} \dot{\theta}+I_{y} \ddot{\theta} \phi=a F_{x F} \delta_{f}+a C_{\alpha F} \alpha_{F}-b C_{\alpha R} \alpha_{R}+M_{z F}+M_{z R}$

Lagrange Equation 3

$\left[I_{x}+m h^{2}+I_{z} \theta^{2}\right] \ddot{\phi}+m h[\dot{v}+u r]+\left[-I_{x} \theta+I_{z} \theta-I_{x z}\right] \dot{r}+\ldots$

$\left[-I_{y}-m h^{2}\right] \phi r^{2}+\left[-I_{x}+I_{z}-I_{y}\right] \dot{\theta} r+\ldots$

$2 I_{z} \dot{\phi} \theta \dot{\theta}+C_{\phi} \dot{\phi}+\left[k_{\phi}-m g h\right] \phi=0$

Lagrange Equation 4

\section{Lagrange Set 4}

The next step is to consider the products of variables terms. Since products of variables are nonlinear and only the linear case is being considered, i.e. $F_{y}=C_{\alpha} \alpha$, it can be assumed that the products of variables are equal to zero. Lagrange equation 1 can be neglected completely because all terms on the left hand side inside the square brackets are product of variables. This leaves:

$0=F_{x F}+F_{x R}-\delta_{f} C_{\alpha F} \alpha_{F}$

Lagrange Equation 1

For the remaining Lagrange equations:

$m \dot{v}+m r u+m h \ddot{\phi}-m h(0)=F_{x F} \delta_{f}+C_{\alpha F} \alpha_{F}+C_{\alpha R} \alpha_{R}$

Lagrange Equation 2 


$$
\begin{aligned}
& m h^{2}(0)+I_{x}(0)+I_{y}(0)+I_{z} \dot{r}+I_{z}(0)-I_{x z} \ddot{\phi}-I_{x}(0)+\ldots \\
& m h(0)+\left[I_{y}+m h^{2}\right] 2(0)+2 I_{x}(0)+\left[I_{z}-I_{x}+I_{y}\right](0)+I_{y}(0)=\ldots \\
& a F_{x F} \delta_{f}+a C_{\alpha F} \alpha_{F}-b C_{\alpha R} \alpha_{R}+M_{z F}+M_{z R}
\end{aligned}
$$

$I_{x} \ddot{\phi}+m h^{2} \ddot{\phi}+I_{z}(0)+m h \dot{v}+m h u r-I_{x}(0)+I_{z}(0)-I_{x z} \dot{r}+\ldots$

$\left[-I_{y}-m h^{2}\right](0)+\left[-I_{x}+I_{z}-I_{y}\right](0)+2 I_{z}(0)+C_{\phi} \dot{\phi}+\left[k_{\phi}-m g h\right] \phi=0$

Lagrange Equation 4

Simplifying:

$$
\begin{array}{lr}
m \dot{v}+m r u+m h \ddot{\phi}=F_{x F} \delta_{f}+C_{\alpha F} \alpha_{F}+C_{\alpha R} \alpha_{R} & \text { Lagrange Equation } 2 \\
I_{z} \dot{r}-I_{x z} \ddot{\phi}=a F_{x F} \delta_{f}+a C_{\alpha F} \alpha_{F}-b C_{\alpha R} \alpha_{R}+M_{z F}+M_{z R} & \text { Lagrange Equation 3 } \\
I_{x} \ddot{\phi}+m h^{2} \ddot{\phi}+m h \dot{v}+m h u r-I_{x z} \dot{r}+C_{\phi} \dot{\phi}+\left[k_{\phi}-m g h\right] \phi=0 & \text { Lagrange Equation 4 }
\end{array}
$$

\section{Lagrange Set 5}

Since the goal is to be able to write these equations in state space form, the following terms will be moved around such that the $\dot{v}, \dot{r}, \dot{\phi}$, and $\ddot{\phi}$ terms are on the left hand side, and the $v, r, \phi$, and $\dot{\phi}$ terms are on the right hand side.

$$
\begin{array}{lr}
m \dot{v}+m h \ddot{\phi}=-m r u+F_{x F} \delta_{f}+C_{\alpha F} \alpha_{F}+C_{\alpha R} \alpha_{R} & \text { Lagrange Equation } 2 \\
I_{z} \dot{r}-I_{x z} \ddot{\phi}=a F_{x F} \delta_{f}+a C_{\alpha F} \alpha_{F}-b C_{\alpha R} \alpha_{R}+M_{z F}+M_{z R} & \text { Lagrange Equation 3 } \\
m h \dot{v}-I_{x z} \dot{r}+C_{\phi} \dot{\phi}+\left[I_{x}+m h^{2}\right] \ddot{\phi}=-m h u r-\left[k_{\phi}-m g h\right] \phi & \text { Lagrange Equation 4 }
\end{array}
$$

\section{Lagrange Set 6}

One final set of substitutions is made. Here, the front and rear tire slip angles, $\alpha_{F}$ and $\alpha_{R}$ respectively, are defined in terms of:

- The front tire steer angle $\delta_{f}$

- The longitudinal and lateral velocities $u$ and $v$, respectively

- The yaw rate $r$ 
- The distance from the front axle to the CG $a$

- The distance from the rear axle to the CG $b$

These equations were derived in Section 4.2 as equations 4.6 and 4.7, and repeated here:

$$
\begin{aligned}
& \alpha_{F}=\delta_{f}-\left(\frac{v+a r}{u}\right) \\
& \alpha_{R}=-\left(\frac{v-b r}{u}\right) \\
& m \dot{v}+m h \ddot{\phi}=-m r u+F_{x F} \delta_{f}+C_{\alpha F}\left[\delta_{f}-\left(\frac{v+a r}{u}\right)\right]+C_{\alpha R}\left[-\left(\frac{v-b r}{u}\right)\right] \\
& I_{z} \dot{r}-I_{x z} \ddot{\phi}=a F_{x F} \delta_{f}+a C_{\alpha F}\left[\delta_{f}-\left(\frac{v+a r}{u}\right)\right]-b C_{\alpha R}\left[-\left(\frac{v-b r}{u}\right)\right]+M_{z F}+M_{z R} \\
& m h \dot{v}-I_{x z} \dot{r}+C_{\phi} \dot{\phi}+\left[I_{x}+m h^{2}\right] \ddot{\phi}=-m h u r-\left[k_{\phi}-m g h\right] \phi
\end{aligned}
$$$$
\text { Lagrange Equation } 2
$$

\section{Lagrange Set 7}

Since these equations will be put into matrix form, it is desirable to combine like terms. Thus, the $C_{\alpha}$ terms will be distributed. Beginning with the Lagrange equation 2:

$$
\begin{aligned}
& m \dot{v}+m h \ddot{\phi}=-m r u+F_{x F} \delta_{f}+C_{\alpha F} \delta_{f}-C_{\alpha F}\left(\frac{v+a r}{u}\right)-C_{\alpha R}\left(\frac{v-b r}{u}\right) \\
& m \dot{v}+m h \ddot{\phi}=-m r u+F_{x F} \delta_{f}+C_{\alpha F} \delta_{f}-C_{\alpha F} \frac{v}{u}-C_{\alpha F} \frac{a r}{u}-C_{\alpha R} \frac{v}{u}+C_{\alpha R} \frac{b r}{u}
\end{aligned}
$$

Now grouping the terms according to the lateral velocity $v$, the yaw rate $r$, and the front tire steer angle $\delta_{f}$ :

$$
m \dot{v}+m h \ddot{\phi}=-\left[\frac{C_{\alpha F}+C_{\alpha R}}{u}\right] v+\left[-m u+\frac{C_{\alpha R} b-C_{\alpha F} a}{u}\right] r+\left[C_{\alpha F}+F_{x F}\right] \delta_{f}
$$


Now for the third Lagrange equation:

$$
\begin{aligned}
& I_{z} \dot{r}-I_{x z} \ddot{\phi}=a F_{x F} \delta_{f}+a C_{\alpha F} \delta_{f}-a C_{\alpha F}\left(\frac{v+a r}{u}\right)-b C_{\alpha R}\left(\frac{b r-v}{u}\right)+M_{z F}+M_{z R} \\
& I_{z} \dot{r}-I_{x z} \ddot{\phi}=a F_{x F} \delta_{f}+a C_{\alpha F} \delta_{f}-a C_{\alpha F} \frac{v}{u}-a C_{\alpha F} \frac{a r}{u}-b C_{\alpha R} \frac{b r}{u}+b C_{\alpha R} \frac{v}{u}+M_{z F}+M_{z R} \\
& I_{z} \dot{r}-I_{x z} \ddot{\phi}=a F_{x F} \delta_{f}+a C_{\alpha F} \delta_{f}-a C_{\alpha F} \frac{v}{u}-C_{\alpha F} \frac{a^{2} r}{u}-C_{\alpha R} \frac{b^{2} r}{u}+b C_{\alpha R} \frac{v}{u}+M_{z F}+M_{z R}
\end{aligned}
$$

Grouping the terms according to the lateral velocity $v$, yaw rate $r$, and the front tire steer angle $\delta_{f}:$

$I_{z} \dot{r}-I_{x z} \ddot{\phi}=\left[\frac{b C_{\alpha R}-a C_{\alpha F}}{u}\right] v-\left[\frac{a^{2} C_{\alpha F}+b^{2} C_{\alpha R}}{u}\right] r+\left[F_{x F}+C_{\alpha F}\right] a \delta_{f}+M_{z F}+M_{z R}$

Lagrange Equation 3

$m h \dot{v}-I_{x z} \dot{r}+C_{\phi} \dot{\phi}+\left[I_{x}+m h^{2}\right] \ddot{\phi}=-m h u r-\left[k_{\phi}-m g h\right] \phi$

Lagrange Equation 4

Finally, the equations can be placed in matrix form:

$$
\begin{aligned}
& {\left[\begin{array}{cccc}
m & 0 & 0 & m h \\
0 & I_{z} & 0 & -I_{x z} \\
0 & 0 & 1 & 0 \\
m h & -I_{x z} & C_{\phi} & {\left[I_{x}+m h^{2}\right]}
\end{array}\right]\left[\begin{array}{c}
\dot{v} \\
\dot{r} \\
\dot{\phi} \\
\ddot{\phi}
\end{array}\right]=\ldots}
\end{aligned}
$$

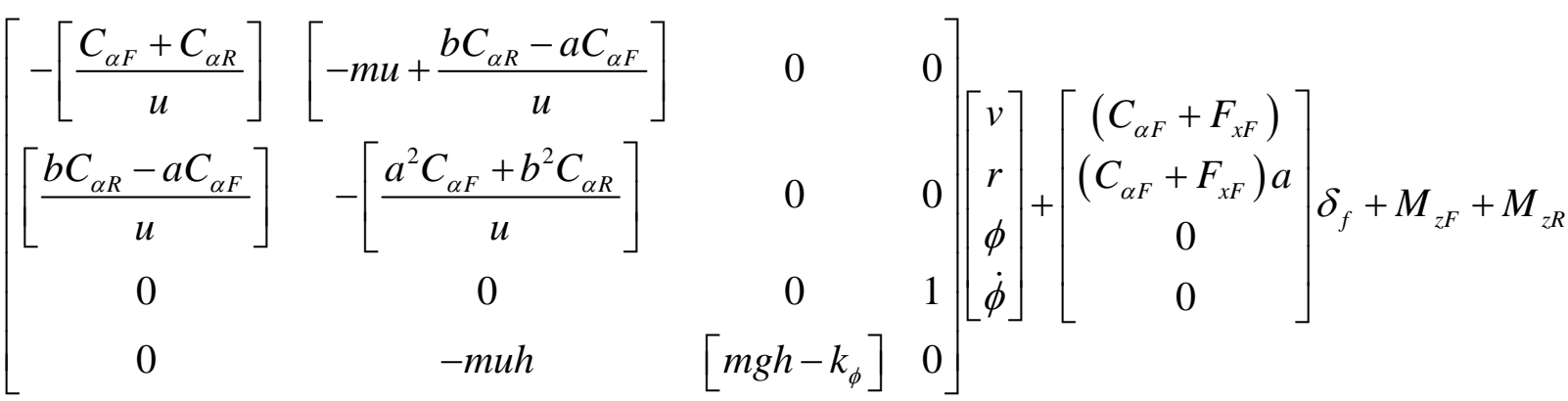

This equation represents the three degree-of-freedom (3DOF) dynamic equation for a two axle vehicle with the parameters defined at the beginning of this chapter. It should be noted that this 
equation contains still contains the inertia cross product $-I_{x z}$; the front longitudinal tire force $F_{x F}$; and the front and rear aligning moments $M_{z F}$ and $M_{z R}$, respectively. If a free rolling vehicle is assumed (i.e. no braking or accelerating); the inertia cross product $-I_{x z}$ is negligible compared to the yaw inertia $I_{z}$ and roll inertia $I_{x}$; and the self aligning moments are small compared to lateral force. Thus, equation 4.210 simplifies to:

$$
\begin{aligned}
& {\left[\begin{array}{cccc}
m & 0 & 0 & m h \\
0 & I_{z} & 0 & 0 \\
0 & 0 & 1 & 0 \\
m h & 0 & C_{\phi} & {\left[I_{x}+m h^{2}\right]}
\end{array}\right]\left[\begin{array}{c}
\dot{v} \\
\dot{r} \\
\dot{\phi} \\
\ddot{\phi}
\end{array}\right]=\ldots}
\end{aligned}
$$

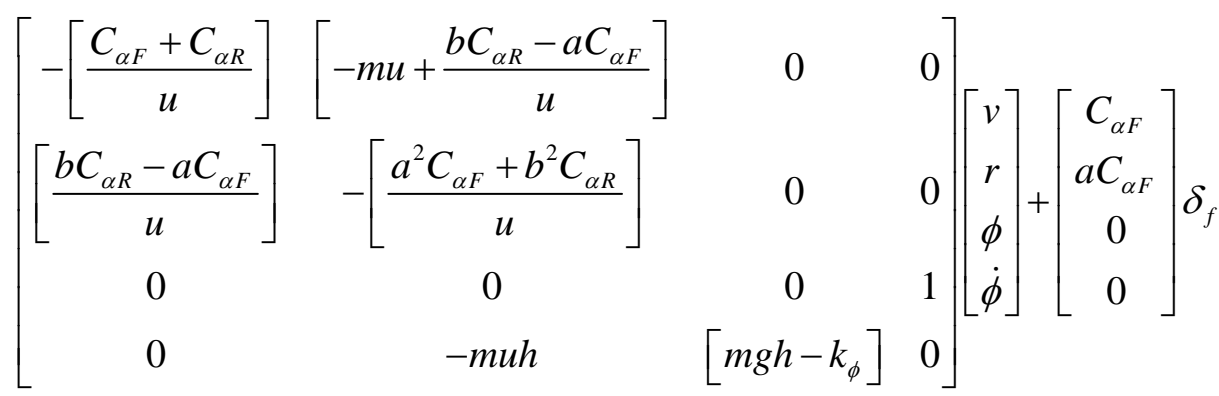

Equation 4.211 is the more familiar form of the three degree-of-freedom (3DOF) equation shown in Pacejka [12].

Since $\sum F_{y}=m a_{y}$ the Lateral Acceleration of the vehicle is defined to be:

$$
\text { Lat_Acc }=\frac{F_{y F}+F_{y R}}{m}
$$

where the numerator is the summation of the lateral tire forces $F_{y F}$ and $F_{y R}$.

\subsection{Future Work for Mathematical Modeling}

Future work involving mathematical modeling could include a two track model as well as longitudinal tire forces and aligning moments. 


\section{Chapter 5}

\section{Vehicle Simulations using Matlab and TruckSim 7®}

Vehicle simulations are an essential part of the development process, whether it is with the vehicle itself [15] or individual vehicle components [25-30]. Essential to any vehicle dynamics simulation is a set of equations that accurately represent the vehicle being tested. Model complexities range from linear, single track two degree-of-freedom (2DOF) bicycle models [713] to models that include eight degrees-of-freedom (8DOF) or more and include nonlinearities such as tire models [18]. Comparisons of these models are often made against real vehicle data but occasionally that is not possible. This was the case for this thesis. Therefore, the Mechanical Simulation Corporation simulation program TruckSim 7® was used for validation of the Matlab simulations, specifically the linear, single track, three degree-of-freedom (3DOF) equation derived in Chapter 4. What follows is a discussion of the Matlab and TruckSim 7® results as well as the development of the final product of this research, the fuzzy logic-based Total Safety Margin (TSM). The Total Safety Margin (TSM) is a stability index and is an attempt to quantify the instability of a vehicle as a single, non-dimensional number.

\subsection{Simulation using the Matlab 3DOF Model and TruckSim 7®}

The list of vehicle stability control maneuvers is vast and includes simple step-steer inputs, accident avoidance maneuvers, decreasing radius turns, J-turn, and Fishhook. The double lane change is probably the most common of the accident avoidance maneuvers. The fuzzy logicbased Total Safety Margin with GPS input developed here is based on the following assumptions:

- The road is a dry, level, high-mu surface.

- The vehicle is symmetric left-to-right.

- No other traffic is present.

- There are no trees, clouds, mountains, or buildings to obstruct the satellite signal.

- The vehicle is traveling in the center of the road.

- The vehicle enters a constant radius turn from straight ahead driving. See Figure 5.1.

- During this constant radius turn the steering wheel angle (SWA) remains at a constant, non-zero value. 
- Currently the radius of curvature is a user-defined value in the simulation, but as described throughout Chapter 3 a real time GPS/INS system combined with a digital road map would be able to read the road ahead and check for changes in radius as illustrated in Figure 5.2. Therefore, this radius input into the Predictive Dynamics Model would result from the GPS/INS signal and digital map (Figure 5.21), and be updated at the GPS refresh rate.

The event chosen was for the vehicle to be traveling in a straight line followed by a single rampsteer input to a constant radius turn. The results of this maneuver were used for determining the Total Safety Margin (TSM). See Figure 5.1.

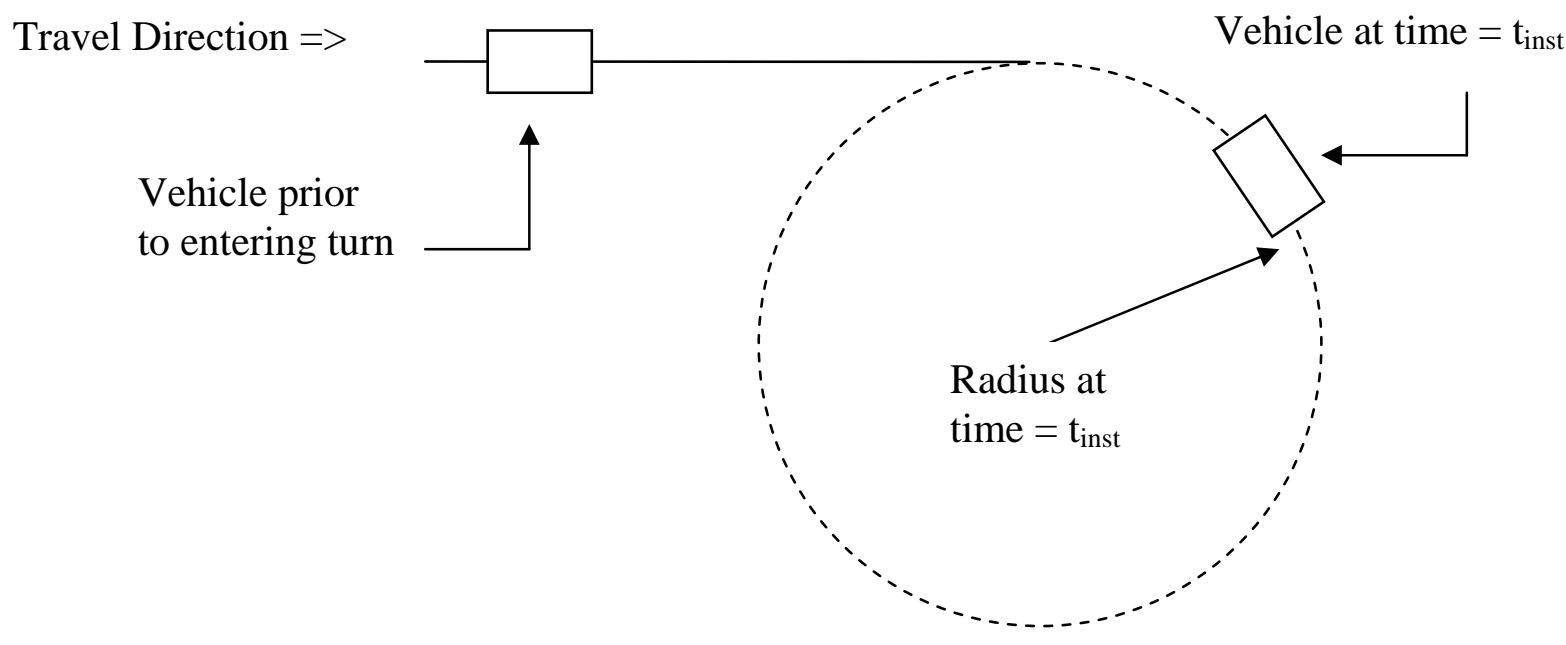

Figure 5.1: Vehicle executing a constant radius turn from straight ahead driving

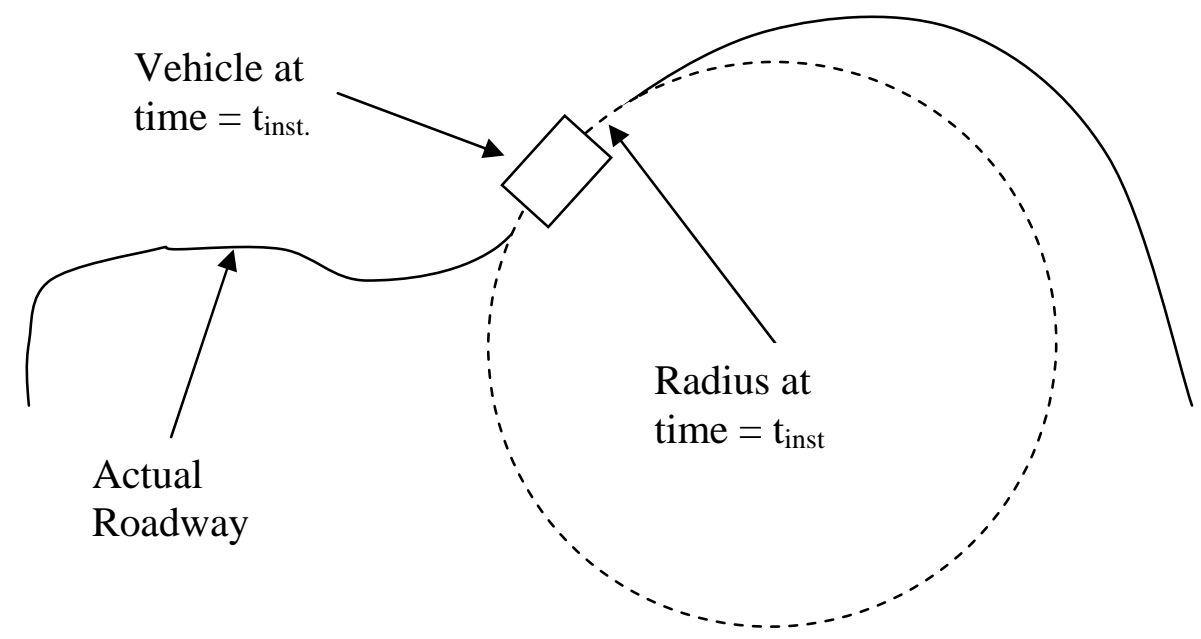

Figure 5.2: Vehicle traveling on a roadway with constantly changing radii 
Simulations were performed for the ramp-steer maneuver described above. The simulated vehicle was tested at three forward velocities:

$$
\begin{aligned}
& 40 \mathrm{kph}=24.9 \mathrm{mph} \\
& 60 \mathrm{kph}=37.3 \mathrm{mph} \\
& 80 \mathrm{kph}=49.7 \mathrm{mph}
\end{aligned}
$$

\section{Table 5.1: Vehicle Simulation Speeds}

Five vehicle mass cases, each simulated at the three speeds in Table 5.1, were chosen to represent a range of commonly traveled commercial vehicle speeds and loading conditions. This testing investigated the operating vehicle dynamics regions. The first vehicle mass was 13172.6 lb $(5987.5 \mathrm{~kg})$, which is the baseline conventional van in TruckSim $7{ }^{\circledR}$ with no load in the cargo box. The maximum vehicle mass simulated was $26000 \mathrm{lb}(11818.2 \mathrm{~kg})$, the assumed Gross Vehicle Weight for a vehicle of this type. The masses between these two limits were chosen to

\begin{tabular}{|c|c|c|c|c|}
\hline \multicolumn{5}{|c|}{ Multimass Calculations } \\
\hline \multicolumn{5}{|c|}{ Vehicle Masses } \\
\hline vehicle mass $_{1}=$ & 13172.6 & $\mathrm{lb}$ & 5987.5 & $\mathrm{~kg}$ \\
\hline vehicle mass $_{2}=$ & 17000.0 & $\mathrm{lb}$ & 7727.3 & $\mathrm{~kg}$ \\
\hline vehicle mass $_{3}=$ & 20000.0 & $\mathrm{lb}$ & 9090.9 & $\mathrm{~kg}$ \\
\hline vehicle mass $_{4}=$ & 23000.0 & lb & 10454.5 & $\mathrm{~kg}$ \\
\hline vehicle mass $_{5}=$ & 26000.0 & $\mathrm{lb}$ & 11818.2 & $\mathrm{~kg}$ \\
\hline
\end{tabular}
best represent various loaded cases (i.e. various total vehicle masses) throughout the assumed mass range. See Table 5.2.

\begin{tabular}{|ccccc|}
\hline \multicolumn{5}{c|}{ Load Masses } \\
& 0.0 & $\mathrm{lb}$ & 0.0 & $\mathrm{~kg}$ \\
\hline $\mathrm{m}_{1 \text { load }}=$ & 3827.4 & $\mathrm{lb}$ & 1739.7 & $\mathrm{~kg}$ \\
$\mathrm{~m}_{2 \text { load }}=$ & 6827.4 & $\mathrm{lb}$ & 3103.4 & $\mathrm{~kg}$ \\
$\mathrm{~m}_{3 \text { load }}=$ & 9827.4 & $\mathrm{lb}$ & 4467.0 & $\mathrm{~kg}$ \\
$\mathrm{~m}_{4 \text { load }}=$ & 12827.4 & $\mathrm{lb}$ & 5830.6 & $\mathrm{~kg}$ \\
$\mathrm{~m}_{5 \text { load }}=$ & & & & \\
\hline
\end{tabular}

Table 5.2 : Vehicle Mass and Load Mass Values 
The test vehicle is shown below in Figure 5.3 with vehicle parameters listed in Appendix B. This vehicle was chosen since it represents a common 2 axle commercial vehicle.

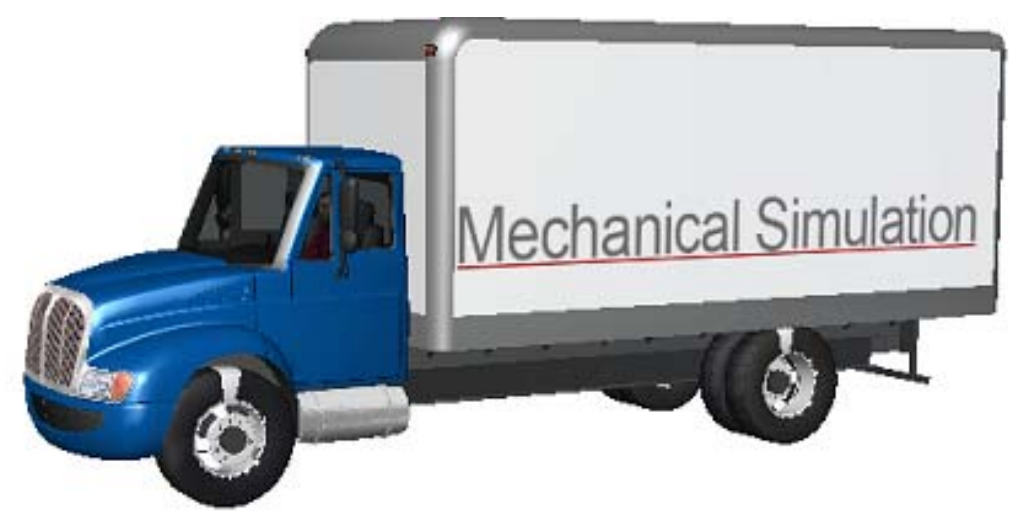

Figure 5.3 : TruckSim Conventional Van, 2 axles [31]

Since the total vehicle mass was increased by increasing the load mass rather than increasing the mass of the truck frame directly at the CG, the vehicle Roll, Pitch, and Yaw inertias changed as well. The parallel axis theorem was used to translate the Roll and Yaw Inertias of the load mass from the load mass' CG to the sprung mass CG of the vehicle. This was needed to accurately predict the Total Safety Margin (TSM). The Pitch moment of inertia is not calculated since Pitch dynamics are not being considered. These inertia properties are summarized in Tables 5.3 - 5.6.

\begin{tabular}{|c|c|c|}
\hline \multicolumn{3}{|c|}{ Inertias of Baseline Vehicle with } \\
Respect to Vehicle Sprung Mass CG \\
\hline $\mathrm{I}_{\mathrm{x}}=$ & 8419.52 & $\mathrm{~kg}-\mathrm{m}^{2}$ \\
\hline $\mathrm{I}_{\mathrm{z}}=$ & 40344.19 & $\mathrm{~kg}-\mathrm{m}^{2}$ \\
\hline
\end{tabular}

Table 5.3 : Inertias of Baseline Vehicle with respect to vehicle sprung mass CG

\begin{tabular}{|c|c|c|c|}
\hline \multicolumn{4}{|c|}{ Inertias of Load with Respect to Load CG } \\
\hline & Roll Inertia & Yaw Inertia & \multirow{2}{*}{} \\
\cline { 2 - 4 } & $\mathrm{I}_{\mathrm{x}}$ & $\mathrm{I}_{\mathrm{z}}$ & \\
\hline Load $_{1}=$ & 0 & 0 & $\mathrm{~kg}-\mathrm{m}^{2}$ \\
Load $_{2}=$ & 795.93 & 1232.31 & $\mathrm{~kg}-\mathrm{m}^{2}$ \\
Load $_{3}=$ & 1419.79 & 2198.21 & $\mathrm{~kg}-\mathrm{m}^{2}$ \\
Load $_{4}=$ & 2043.65 & 3164.13 & $\mathrm{~kg}-\mathrm{m}^{2}$ \\
Load $_{5}=$ & 2667.52 & 4130.04 & $\mathrm{~kg}-\mathrm{m}^{2}$ \\
\hline
\end{tabular}

Table 5.4 : Summary of Load Inertias 


\begin{tabular}{|c|c|c|c|c|}
\hline \multicolumn{5}{|c|}{ Inertias of Load with Respect to Vehicle Spung Mass CG } \\
\hline \multicolumn{3}{|c|}{$\begin{array}{l}x \text { distance b/w load CG and SMCG: } \\
z \text { distance b/w load CG and SMCG: }\end{array}$} & $\begin{array}{l}3.387 \\
0.927\end{array}$ & $\begin{array}{l}\mathrm{m} \\
\mathrm{m}\end{array}$ \\
\hline & Roll Inertia & Yaw Inertia & & \\
\hline & $\mathrm{I}_{\mathrm{x}}$ & $\mathrm{I}_{\mathrm{z}}$ & & \\
\hline $\operatorname{Load}_{1}=$ & 0 & 0 & $\mathrm{~kg}-\mathrm{m}^{2}$ & \\
\hline $\operatorname{Load}_{2}=$ & 2290.93 & 21190.06 & $\mathrm{~kg}-\mathrm{m}^{2}$ & \\
\hline $\operatorname{Load}_{3}=$ & 4086.60 & 37799.28 & $\mathrm{~kg}-\mathrm{m}^{2}$ & \\
\hline $\operatorname{Load}_{4}=$ & 5882.27 & 54408.52 & $\mathrm{~kg}-\mathrm{m}^{2}$ & \\
\hline $\operatorname{Load}_{5}=$ & 7677.95 & 71017.75 & $\mathrm{~kg}-\mathrm{m}^{2}$ & \\
\hline
\end{tabular}

Table 5.5 : Summary of Inertias of Load with respect to vehicle sprung mass CG

The following expression can be used:

Inertia of Loaded Vehicle = Inertia of Baseline Vehicle with respect to vehicle sprung mass CG + Inertia of Load with respect to vehicle sprung mass CG

\begin{tabular}{|c|c|c|c|c|c|c|}
\hline & \multicolumn{3}{|c|}{ Roll Inertia, kg-m² } & \multicolumn{3}{|c|}{ Yaw Inertia, kg-m² } \\
\hline & $\mathrm{I}_{\mathrm{x}, \text { Base vehicle }}$ & $\mathrm{I}_{\mathrm{x}, \text { Load }}$ & $\mathrm{I}_{\mathrm{x}, \text { Total }}$ & $I_{z, \text { Base Vehicle }}$ & $\mathrm{I}_{z, \text { Load }}$ & $\mathrm{I}_{\mathrm{z}, \text { Total }}$ \\
\hline vehicle mass $_{1}=$ & 8419.52 & 0.00 & 8419.52 & 40344.19 & 0 & 40344.19 \\
\hline vehicle mass $_{2}=$ & 8419.52 & 2290.93 & 10710.44 & 40344.19 & 21190.06 & 61534.25 \\
\hline vehicle mass $_{3}=$ & 8419.52 & 4086.60 & 12506.12 & 40344.19 & 37799.28 & 78143.47 \\
\hline vehicle mass $_{4}=$ & 8419.52 & 5882.27 & 14301.79 & 40344.19 & 54408.52 & 94752.72 \\
\hline vehicle mass $_{5}=$ & 8419.52 & 7677.95 & 16097.47 & 40344.19 & 71017.75 & 111361.95 \\
\hline
\end{tabular}

Table 5.6 : Summary of Total Vehicle Roll and Yaw Inertias with respect to vehicle sprung mass CG

One significant area of research concentrated on determining the tire cornering stiffnesses. Since a tire's ability to generate longitudinal and lateral forces are based upon, among other things, the tire normal load and tire slip angle, it made sense to supply the Matlab simulation with cornering stiffness values for each mass case. Initially, the tire cornering stiffness properties were taken from the Absolute Lateral Force vs. Slip Angle graphs available within TruckSim at a single slip angle value. Since the steering wheel angle is steered to a maximum value of 52 degrees in the simulated maneuver, corresponding to a $152.4 \mathrm{~m}(500 \mathrm{ft})$ radius turn, and the vehicle has a steering gear ratio of $25: 1$, the maximum front tire slip angle is $2.08 \mathrm{deg}$. Truncating this value to two degrees, the corresponding lateral force was chosen for each of the tire normal loads at a slip angle of two degrees. 
The default TruckSim tire used was an $\mathrm{F}_{\mathrm{y}}=3000 \mathrm{~kg}$ load rated tire and the Absolute Lateral Force vs. Slip Angle plot is shown in Figure 5.4. This plot contains data for five normal loads. Since the vehicle used in this study was tested at five loading conditions and not all of the individual tire normal loads were represented on this plot, the TruckSim tire data was exported to Excel and then plotted in Matlab. The lateral forces at the new normal loads were then calculated and added to these plots in Matlab using an interpolation routine.

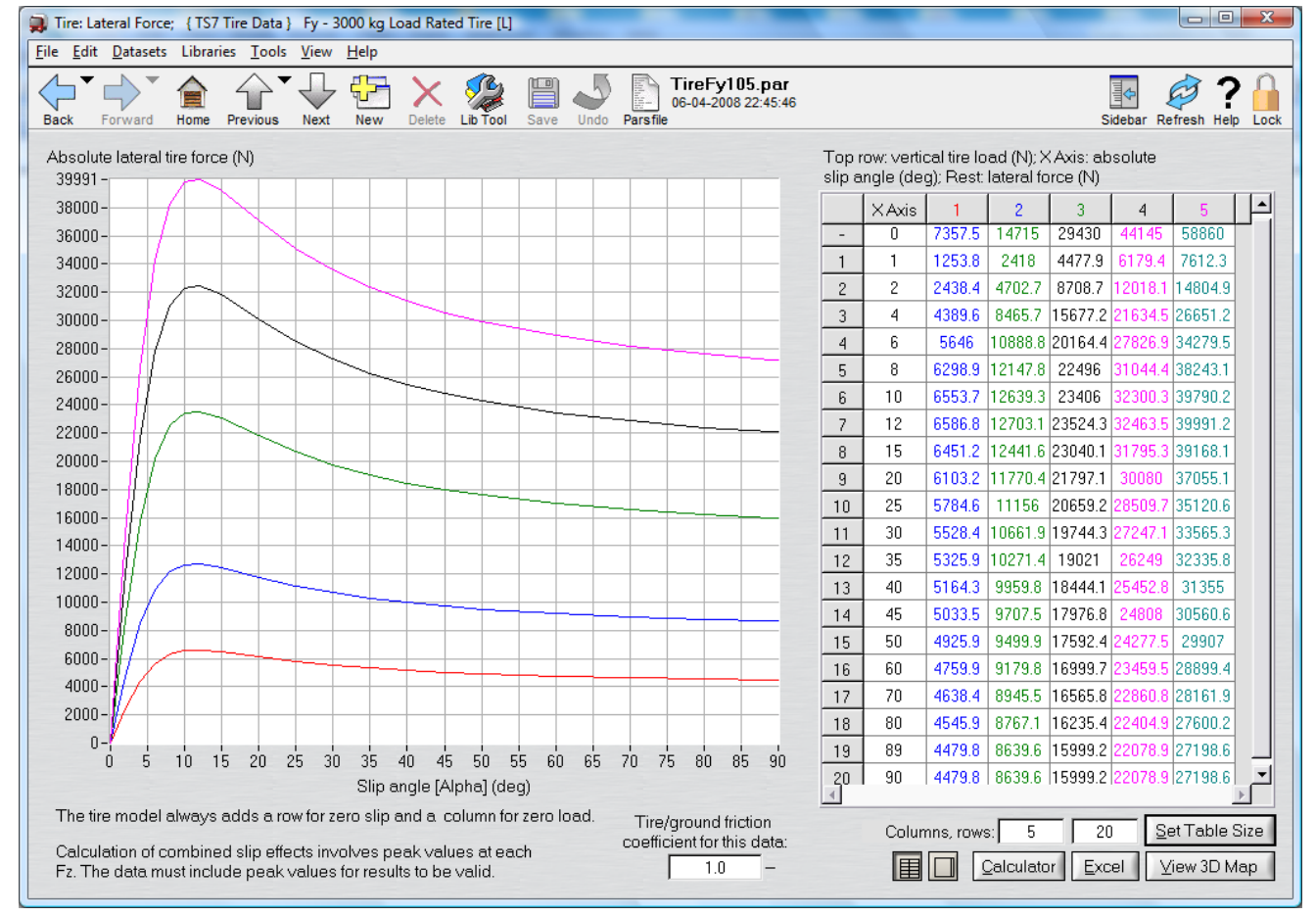

Figure 5.4 : TruckSim Tire Lateral Force vs. Slip Angle for $3000 \mathrm{~kg}$ Load Rated Tire [31]

The TruckSim Open Loop Steering Control was used to control the steering wheel angle input for the TruckSim vehicle. This is shown in Figure 5.5a. A similar steering wheel angle plot is used for the 3DOF Matlab vehicle using a slightly smoothed step function. These two are shown together in Figure 5.5b. 


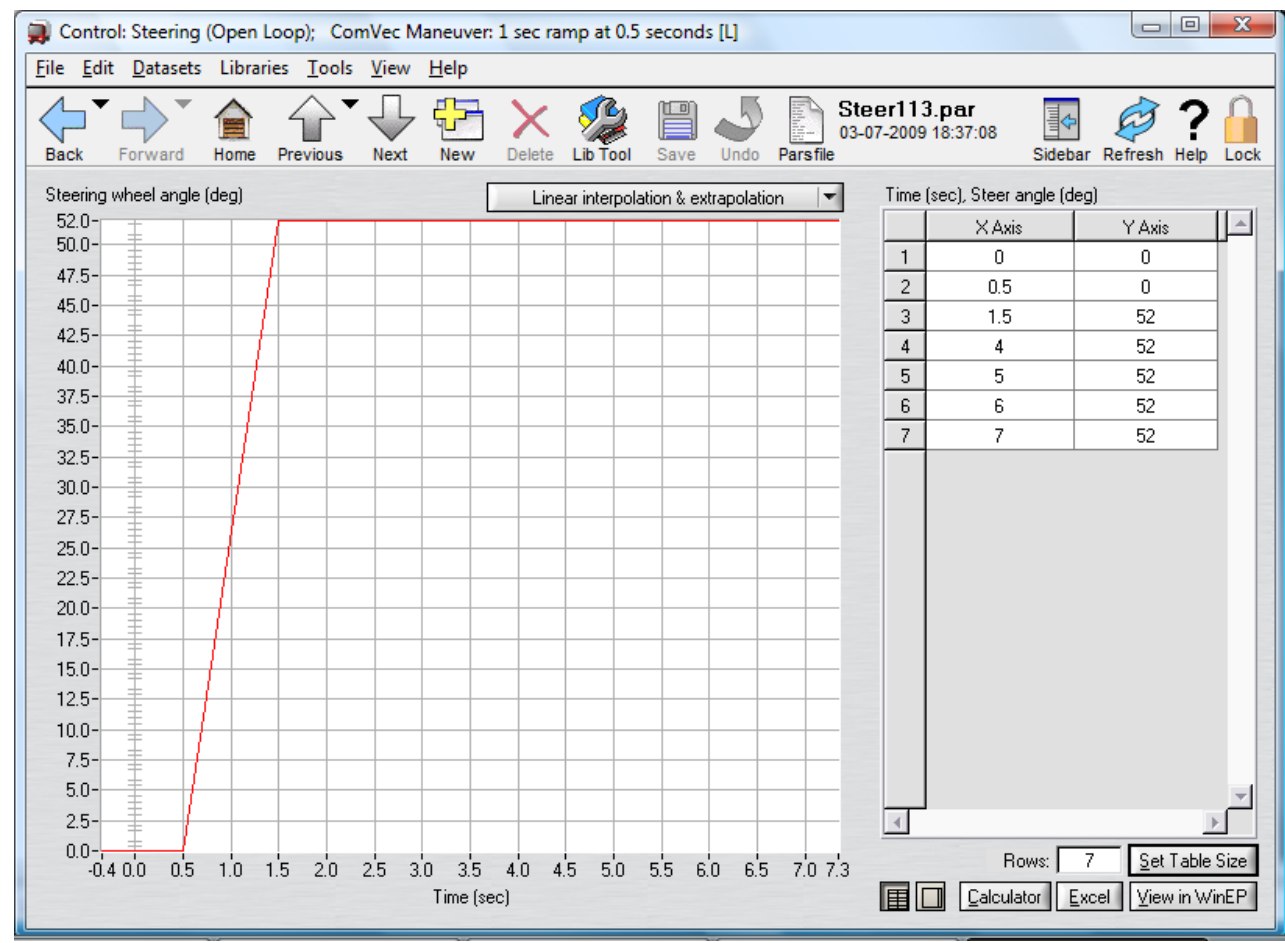

Figure 5.5a : TruckSim Open Loop Steering Controller [31]

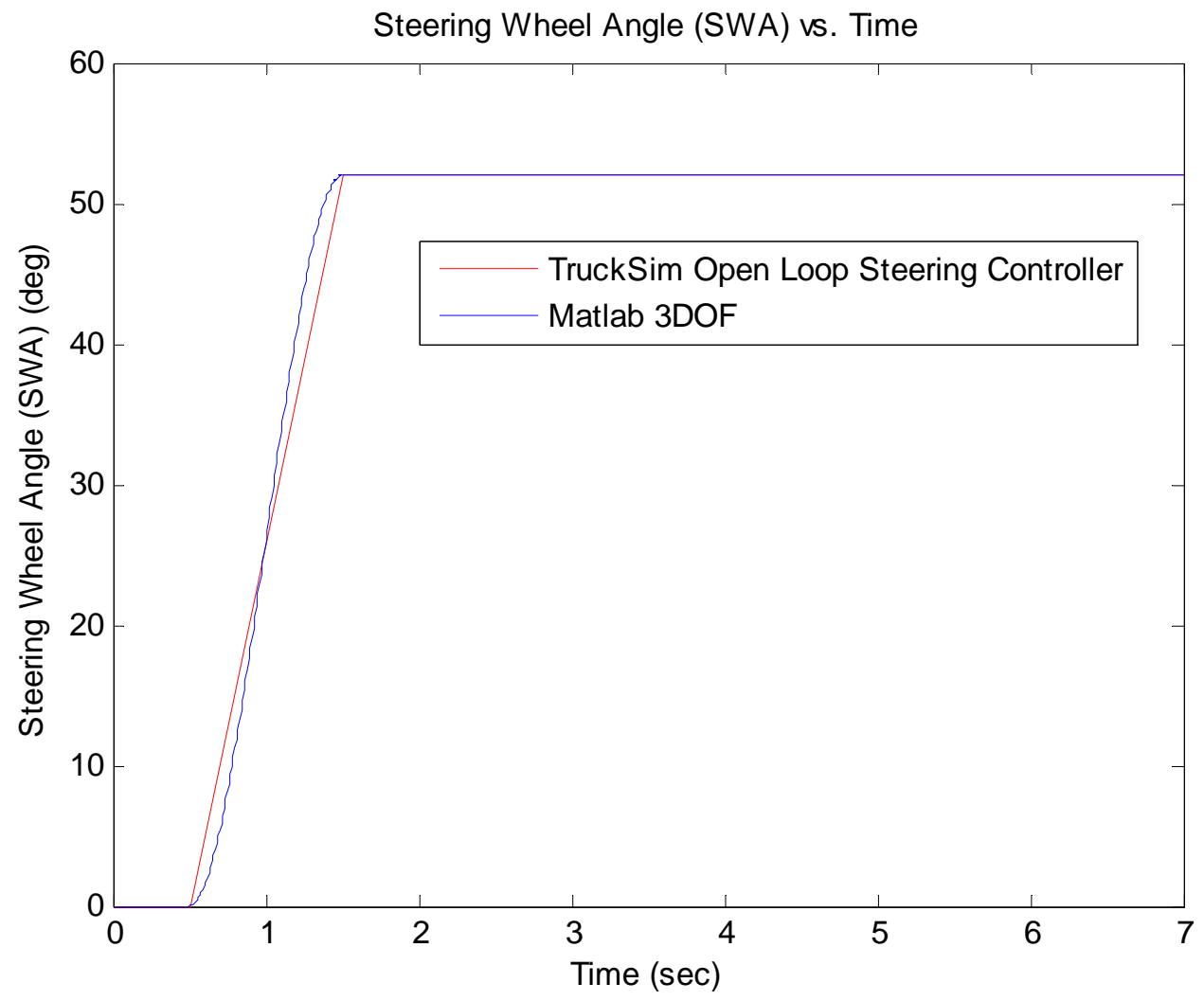

Figure 5.5b : TruckSim Steering Controller and Matlab 3DOF smoothed step 
Initial comparisons between Matlab and TruckSim using these TruckSim-based lateral force values indicated some counterintuitive behavior [24]. In the Matlab simulations the heaviest vehicle showed the lowest response values for Lateral Acceleration and Yaw Rate, while TruckSim showed the opposite. Several sets of cornering stiffnesses were tried but the Matlab results did not match TruckSim very closely.

Based on the author's previous work experience with Honda R\&D Americas, Inc. and Continental-Teves, as well as conversations with Dr. Tom Gillespie [33], Lateral Acceleration and Yaw Rate, followed by Roll Angle, were the three most important vehicle responses to study when investigating vehicle dynamics, especially when rollover is a potential factor. Therefore it was decided to compare the Lateral Acceleration and Yaw Rate plots from Matlab and TruckSim and calibrate the rear tire cornering stiffnesses until the vehicle responses were as close as possible.

This procedure was performed and based on the simulation results it was determined this process was successful. The resulting tire cornering stiffnesses are shown in Table 5.7.

\begin{tabular}{|c|c|c|c|}
\hline \multicolumn{4}{|c|}{$\begin{array}{c}\text { Tire Cornering Stiffness } C_{\alpha} \text {, Summary } \\
\text { values are per tire }\end{array}$} \\
\hline & Front & Rear & Unit \\
\hline vehicle mass $_{1}$ & 198043 & 36684 & $\mathrm{~N} / \mathrm{rad}$ \\
\hline vehicle mass $_{2}$ & 249494 & 44307 & $\mathrm{~N} / \mathrm{rad}$ \\
\hline vehicle mass $_{3}$ & 296907 & 52623 & $\mathrm{~N} / \mathrm{rad}$ \\
\hline vehicle mass $_{4}$ & 316846 & 55095 & $\mathrm{~N} / \mathrm{rad}$ \\
\hline 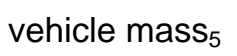 & 344348 & 59301 & $\mathrm{~N} / \mathrm{rad}$ \\
\hline
\end{tabular}

Table 5.7 : Final Tire Cornering Stiffness Values

Note: These are per tire. Since a single track model is being used and the vehicle has a dually real axle, the front tire cornering stiffness is multiplied by two and the rear tire cornering stiffness is multiplied by four in the Matlab simulation.

The following conditions were used:

$$
\begin{array}{llll}
u=40,60,80 \mathrm{kph} & S W A(0)=0^{0} & v(0)=0 \mathrm{kph} & \phi(0)=0 \mathrm{rad} \\
R=152.4 m & S W A_{-} \text {max }=52^{0} & r(0)=0 \frac{\mathrm{rad}}{\mathrm{s}} & \dot{\phi}(0)=0 \frac{\mathrm{rad}}{\mathrm{s}}
\end{array}
$$

Lat_Acc $(0)=0 g$

Initial simulations were performed with the unloaded baseline vehicle at each of the three speeds listed in Table 5.1. This was done to check the functionality of the Matlab simulation and establish the baseline vehicle dynamics response levels. The steady state results are summarized in Table 5.8 and plotted in Figures 5.6 - 5.8. 


\begin{tabular}{|c|c|c|c|c|}
\hline \multicolumn{5}{|c|}{ Summary of Steady State Values } \\
\hline \multicolumn{5}{|c|}{ Baseline Vehicle, no load } \\
\hline $\begin{array}{c}\text { Speed } \\
\text { kph }\end{array}$ & $\begin{array}{c}\text { Lat_Acc } \\
\text { g }\end{array}$ & $\begin{array}{c}\text { Lat_Vel } \\
\text { kph }\end{array}$ & $\begin{array}{c}\text { Yaw Rate } \\
\text { deg/s }\end{array}$ & $\begin{array}{c}\text { Roll Angle } \\
\text { deg }\end{array}$ \\
\hline 40 & 0.0857 & 0.7535 & 4.336 & -0.4803 \\
60 & 0.1790 & 0.5173 & 6.038 & -1.003 \\
80 & 0.2892 & -0.2753 & 7.316 & -1.621 \\
\hline
\end{tabular}

Table 5.8 : Summary of Steady State Values for Baseline Vehicle, no load
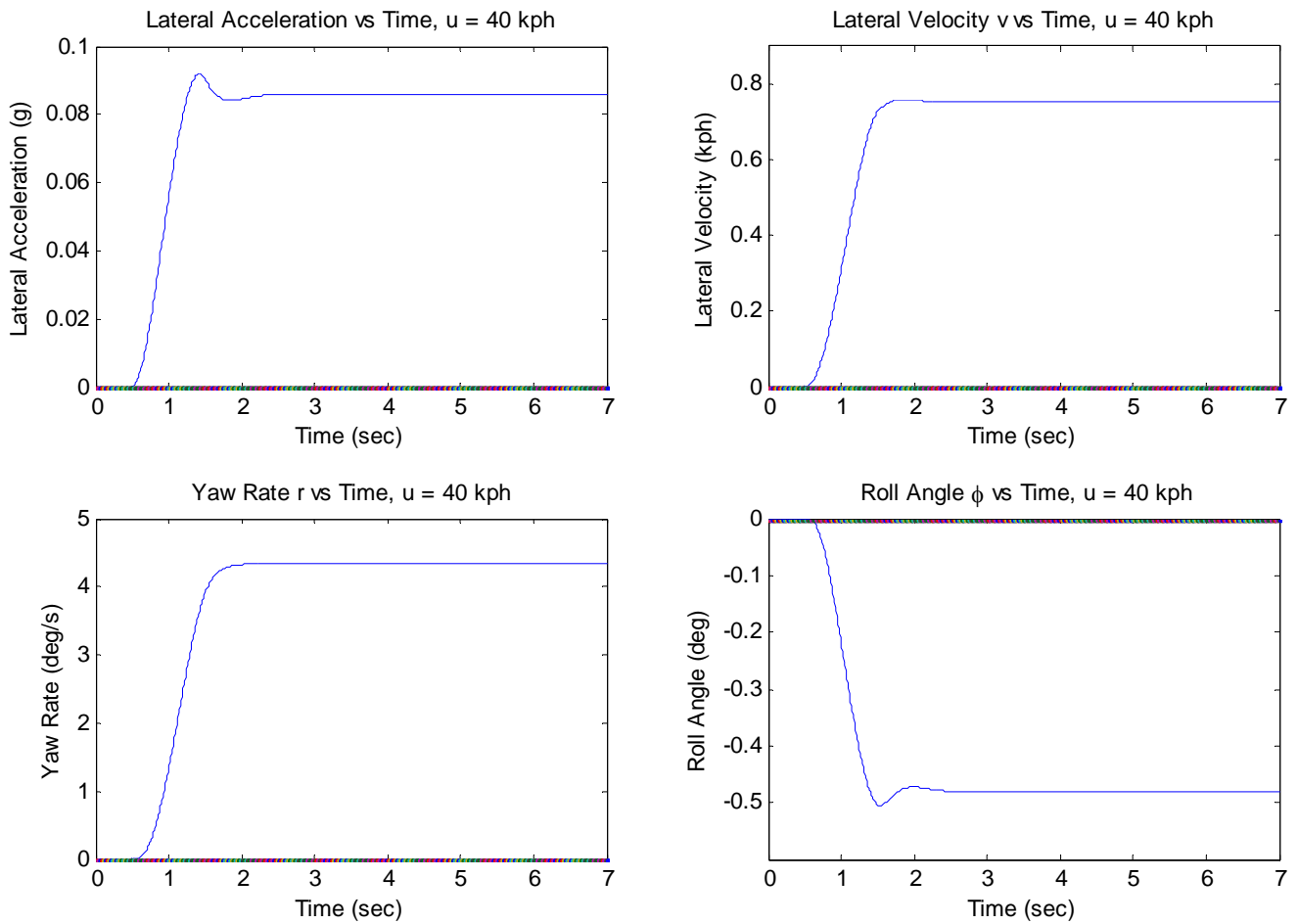

Figure 5.6: 3DOF baseline vehicle at $13172.6 \mathrm{lb}$ and no cargo load at $\mathrm{u}=40 \mathrm{kph}$ 

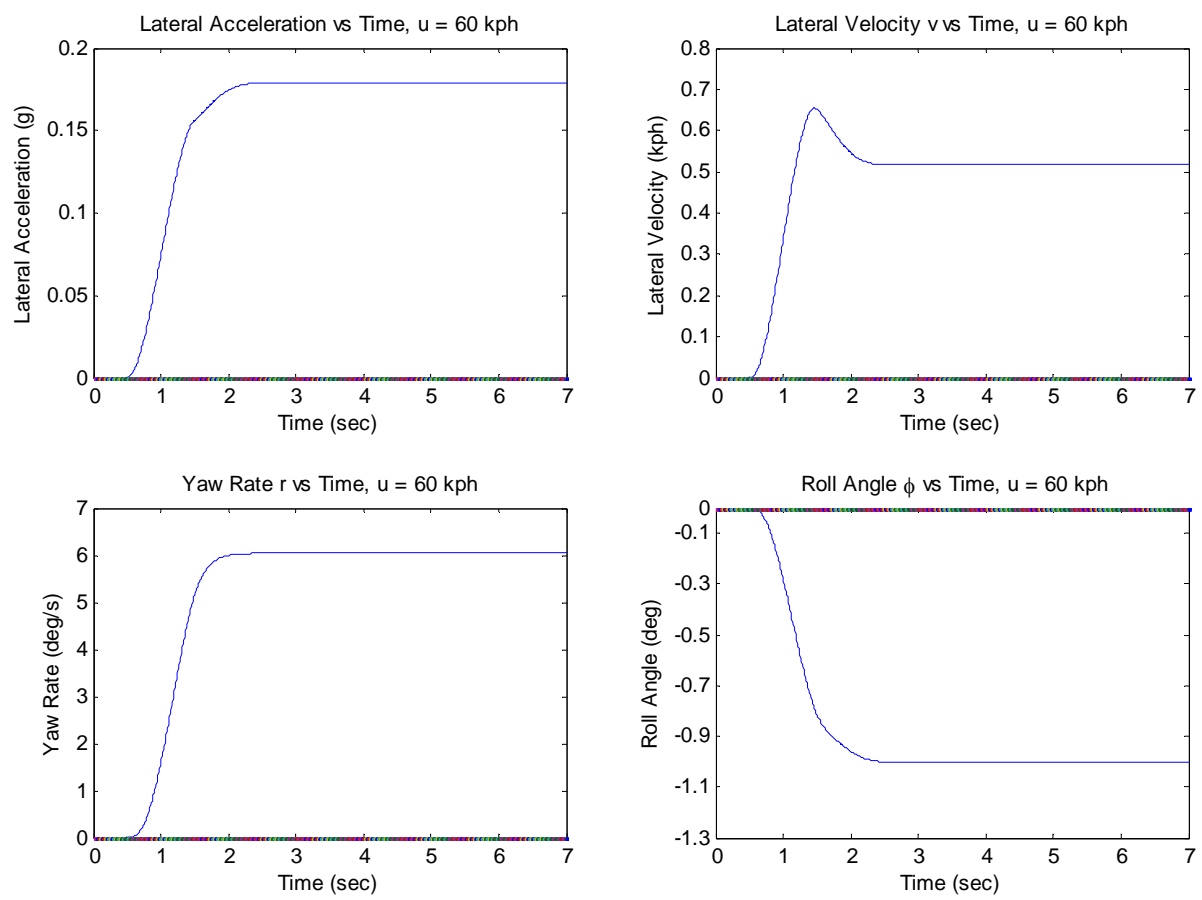

Figure 5.7 : 3DOF baseline vehicle at $13172.6 \mathrm{lb}$ and no cargo load at $\mathrm{u}=60 \mathrm{kph}$
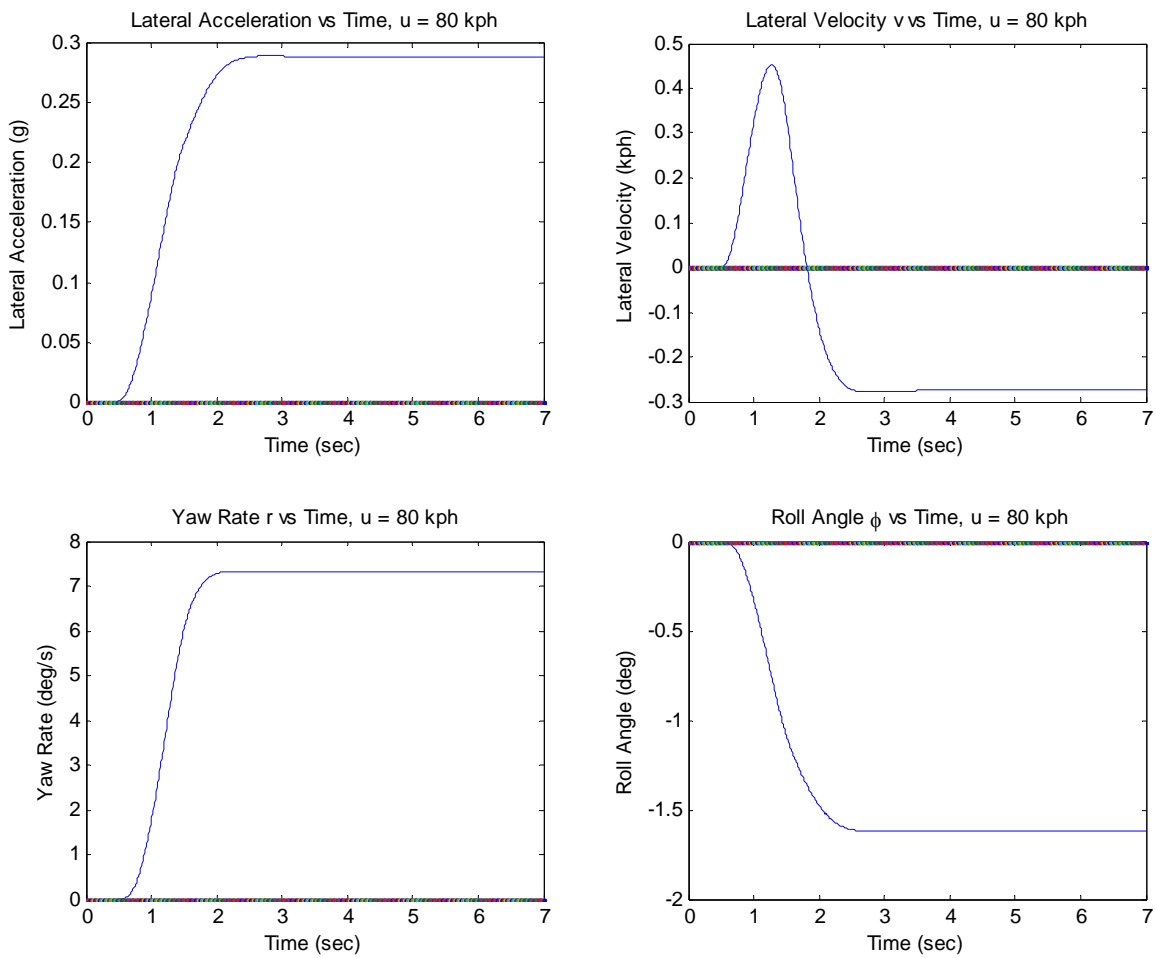

Figure 5.8 : 3DOF baseline vehicle at $13172.6 \mathrm{lb}$ and no cargo load at $\mathrm{u}=80 \mathrm{kph}$ 
The lateral velocity plot at $80 \mathrm{kph}$ shown in Figure 5.8 is worth some discussion. Compared to the lateral velocities at $40 \mathrm{kph}$ and $60 \mathrm{kph}$ shown in Figures 5.6 and 5.7, the lateral velocity changes sign in the $80 \mathrm{kph}$ test. As shown in Figures 4.1, 4.10, and 4.11, the SAE vehicle coordinate system defines the positive lateral direction out the right side of the vehicle. Since the simulated maneuver has the vehicle performing a right turn and the lateral velocity is positive in the $40 \mathrm{kph}$ and $60 \mathrm{kph}$ tests, this would indicate the vehicle is translating positively to the right as it moves longitudinally. Such a behavior might be described as crabbing. Therefore, this indicates that since the lateral velocity changes sign, i.e. pointing in the positive $y$ direction to the inside of the turn, to negative, indicating it now points to the outside of the turn, the rear tire lateral forces must also change sign. The vehicle velocity vector would also change direction, from initially pointing inside the turn radius to outside of it as lateral velocity changes direction.

One possible explanation for this behavior is to consider the lateral displacement of the rear wheel hubs with respect to the rear tire contact patches. In straight ahead driving, assume that the rear hubs and rear tire contact patches are aligned, i.e. the tires are not laterally deflected. The front wheels are steered which initiates the turn. When this happens, the rear wheel hubs initially translate towards the inside of the turn. This happens as the entire vehicle initially translates laterally in the direction that the front wheels are steered. The tire contact patch remains close to its original position due to the adhesion it has with the road surface. Therefore, the rear tires are now deflected laterally. In the $40 \mathrm{kph}$ and $60 \mathrm{kph}$ cases illustrated above, this is where the lateral tire deflection discussion ends.

For the $80 \mathrm{kph}$ case, though, as the turn continues and the vehicle takes a steady state cornering orientation which includes the vehicle translating laterally to the outside of the turn, the rear wheel hubs also translate laterally with respect to the tire contact patch. However, they have now translated in the opposite direction they did originally. This new lateral tire deflection is where the tire lateral force would change sign. Further investigation is necessary to test this theory.

The next step was to extend this testing to include all five of the mass cases, the results of which are shown below in Tables $5.9-5.10$ and Figures 5.9 - 5.15. As stated above, TruckSim was used as the validation tool and this set of tests determined the operating vehicle dynamics regions. 


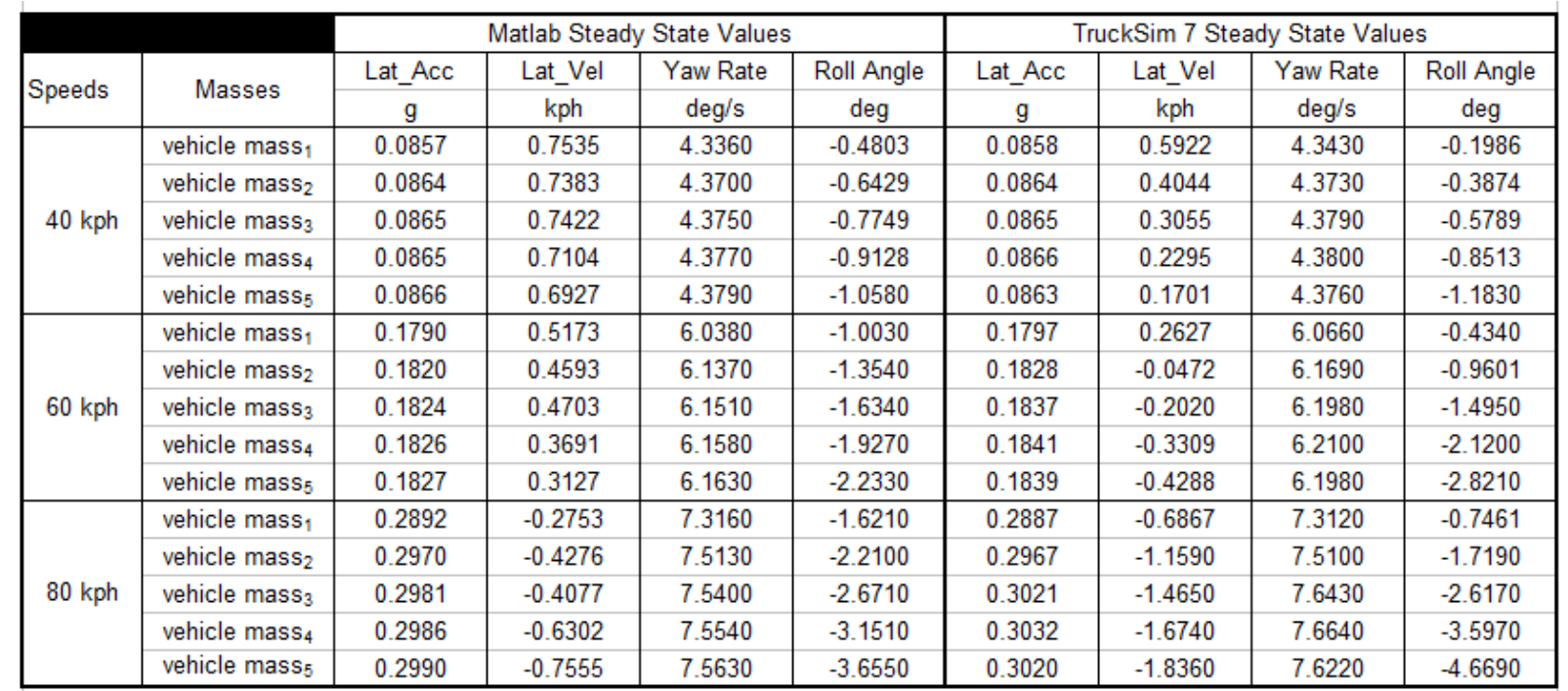

Table 5.9 : Summary of Matlab and TruckSim Multimass Studies

\begin{tabular}{|c|c|c|c|c|c|c|c|c|c|}
\hline \multirow[b]{3}{*}{ Speeds } & \multirow[b]{3}{*}{ Masses } & \multicolumn{8}{|c|}{ Percent Error and Difference - Multimass Study, Steady State Values } \\
\hline & & \multicolumn{2}{|c|}{ Lat_Acc (g) } & \multicolumn{2}{|c|}{ Lat_Vel (kph) } & \multicolumn{2}{|c|}{ Yaw Rate (deg/s) } & \multicolumn{2}{|c|}{ Roll Angle (deg) } \\
\hline & & $\%$ Error & $\begin{array}{c}\text { Difference = } \\
(\text { TS7-Matlab) }\end{array}$ & $\%$ Error & $\begin{array}{l}\text { Difference = } \\
(\text { TS7-Matlab) }\end{array}$ & $\%$ Error & $\begin{array}{l}\text { Difference = } \\
(\text { TS7-Matlab) }\end{array}$ & $\%$ Error & $\begin{array}{l}\text { Difference = } \\
\text { (TS7-Matlab) }\end{array}$ \\
\hline \multirow{5}{*}{$40 \mathrm{kph}$} & vehicle mass $_{1}$ & 0.13 & 0.0001 & 27.24 & -0.1613 & 0.16 & 0.0070 & 141.84 & 0.2817 \\
\hline & vehicle mass $_{2}$ & 0.03 & 0.0000 & 82.57 & -0.3339 & 0.07 & 0.0030 & 65.95 & 0.2555 \\
\hline & vehicle mass $_{3}$ & 0.07 & 0.0001 & 142.95 & -0.4367 & 0.09 & 0.0040 & 33.86 & 0.1960 \\
\hline & vehicle mass $_{4}$ & 0.05 & 0.0000 & 209.54 & -0.4809 & 0.07 & 0.0030 & 7.22 & 0.0615 \\
\hline & vehicle mass $_{5}$ & 0.30 & -0.0003 & 307.23 & -0.5226 & 0.07 & -0.0030 & 10.57 & -0.1250 \\
\hline \multirow{5}{*}{60 kph } & vehicle mass $_{1}$ & 0.39 & 0.0007 & 96.92 & -0.2546 & 0.46 & 0.0280 & 131.11 & 0.5690 \\
\hline & vehicle mass $_{2}$ & 0.44 & 0.0008 & 1073.09 & -0.5065 & 0.52 & 0.0320 & 41.03 & 0.3939 \\
\hline & vehicle mass $_{3}$ & 0.71 & 0.0013 & 332.82 & -0.6723 & 0.76 & 0.0470 & 9.30 & 0.1390 \\
\hline & vehicle mass $_{4}$ & 0.81 & 0.0015 & 211.54 & -0.7000 & 0.84 & 0.0520 & 9.10 & -0.1930 \\
\hline & 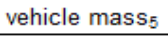 & 0.65 & 0.0012 & 172.92 & -0.7415 & 0.56 & 0.0350 & 20.84 & -0.5880 \\
\hline \multirow{5}{*}{80 kph } & 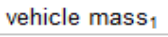 & 0.17 & -0.0005 & 59.91 & -0.4114 & 0.05 & -0.0040 & 117.26 & 0.8749 \\
\hline & vehicle mass $_{2}$ & 0.10 & -0.0003 & 63.11 & -0.7314 & 0.04 & -0.0030 & 28.56 & 0.4910 \\
\hline & vehicle mass $_{3}$ & 1.32 & 0.0040 & 72.17 & -1.0573 & 1.35 & 0.1030 & 2.06 & 0.0540 \\
\hline & vehicle mass $_{4}$ & 1.52 & 0.0046 & 62.35 & -1.0438 & 1.44 & 0.1100 & 12.40 & -0.4460 \\
\hline & vehicle mass $_{5}$ & 0.99 & 0.0030 & 58.85 & -1.0805 & 0.77 & 0.0590 & 21.72 & -1.0140 \\
\hline
\end{tabular}

Table 5.10 : Percent Error and Difference for Multimass Cases, Steady State Values

As can be seen from Tables 5.9 - 5.10, the lateral acceleration and yaw rate results compare very well. For example, the lowest percent errors are for the Lateral Acceleration and Yaw Rate cases at all three speeds. This can be attributed to the cornering stiffness calibration procedure.

However, the Roll Angle and Lateral Velocity results do not compare that well. For example, for Lateral Velocity the Matlab vehicle was consistently larger for the $40 \mathrm{kph}$ case and lagged behind the TruckSim vehicle at $60 \mathrm{kph}$ and $80 \mathrm{kph}$. Percent errors ranged from $27.24 \%$ (vehicle mass $_{1}$ at $40 \mathrm{kph}$ ) to $1073.09 \%$ (vehicle mass 2 at $60 \mathrm{kph}$ ). To put this in perspective, for the 27.24 $\%$ error, the Matlab vehicle was $0.1613 \mathrm{kph}$ faster laterally than the TruckSim vehicle. For the $1073.09 \%$ error, the Matlab vehicle was $0.5065 \mathrm{kph}$ slower than the TruckSim vehicle. 
Similar commentary can be made for the Roll Angle. The lowest percent error was $2.06 \%$ (vehicle mass $_{3}$, $80 \mathrm{kph}$ ) while the largest was $141.84 \%$ (vehicle mass $1,40 \mathrm{kph}$ ). For the $2.06 \%$ error, the Matlab vehicle rolled 0.0540 deg more than the TruckSim vehicle. For the $141.84 \%$ error, the Matlab vehicle rolled 0.2817 deg more than the TruckSim vehicle.

Further investigation would have to be performed to see how significant these errors are.

$$
\begin{aligned}
\text { - vehicle } \text { mass }_{1} & =13172.6 \mathrm{lb} \\
- \text { vehicle } \text { mass }_{2} & =17000 \mathrm{lb} \\
- \text { vehicle } \text { mass }_{3} & =20000 \mathrm{lb} \\
- \text { vehicle } \text { mass }_{4} & =23000 \mathrm{lb} \\
\text { - vehicle } \text { mass }_{5} & =26000 \mathrm{lb}
\end{aligned}
$$

Figure 5.9 : Legend for multimass studies 

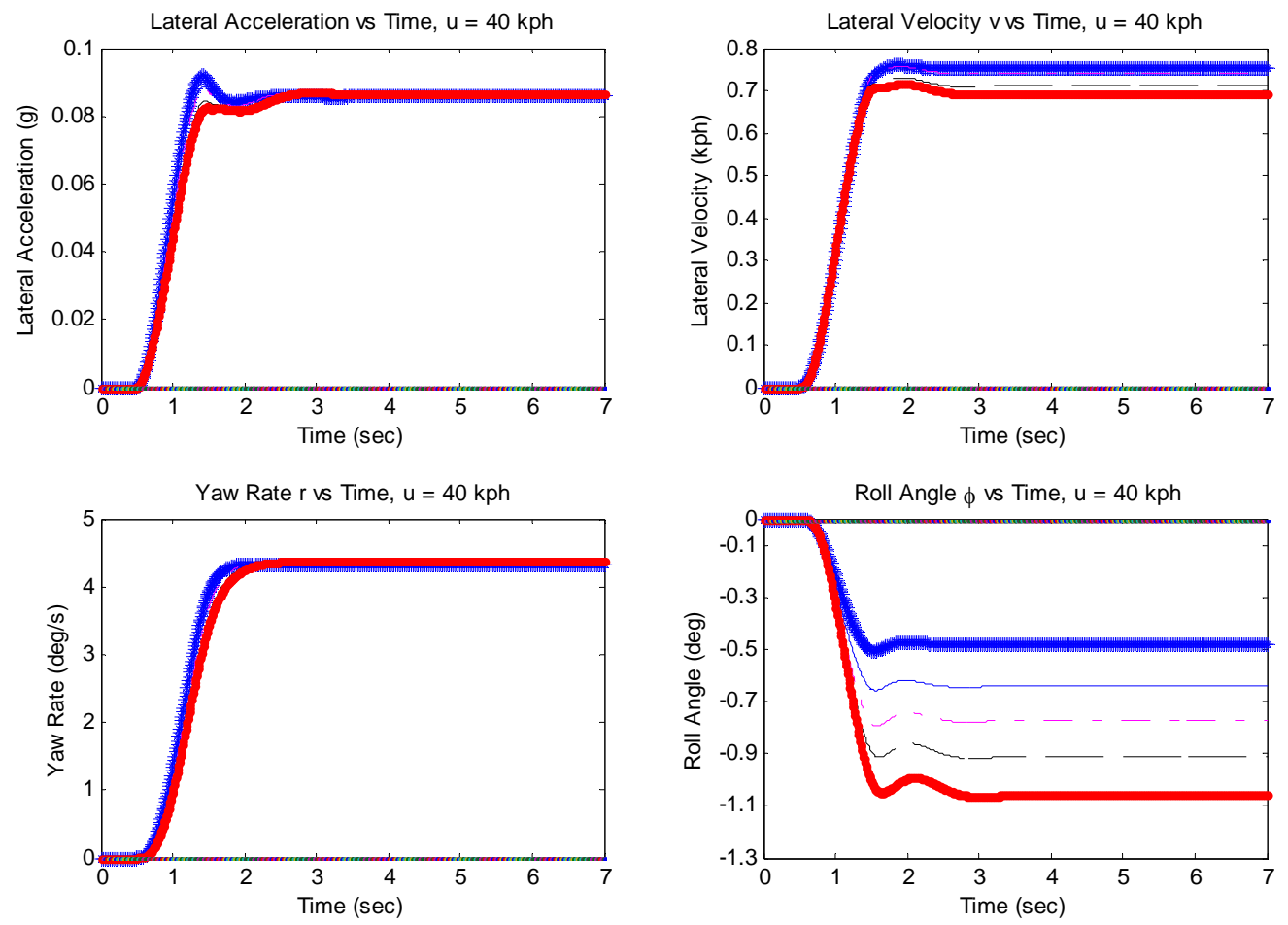

Figure 5.10 : 3DOF multimass vehicle study at $\mathrm{u}=40 \mathrm{kph}$
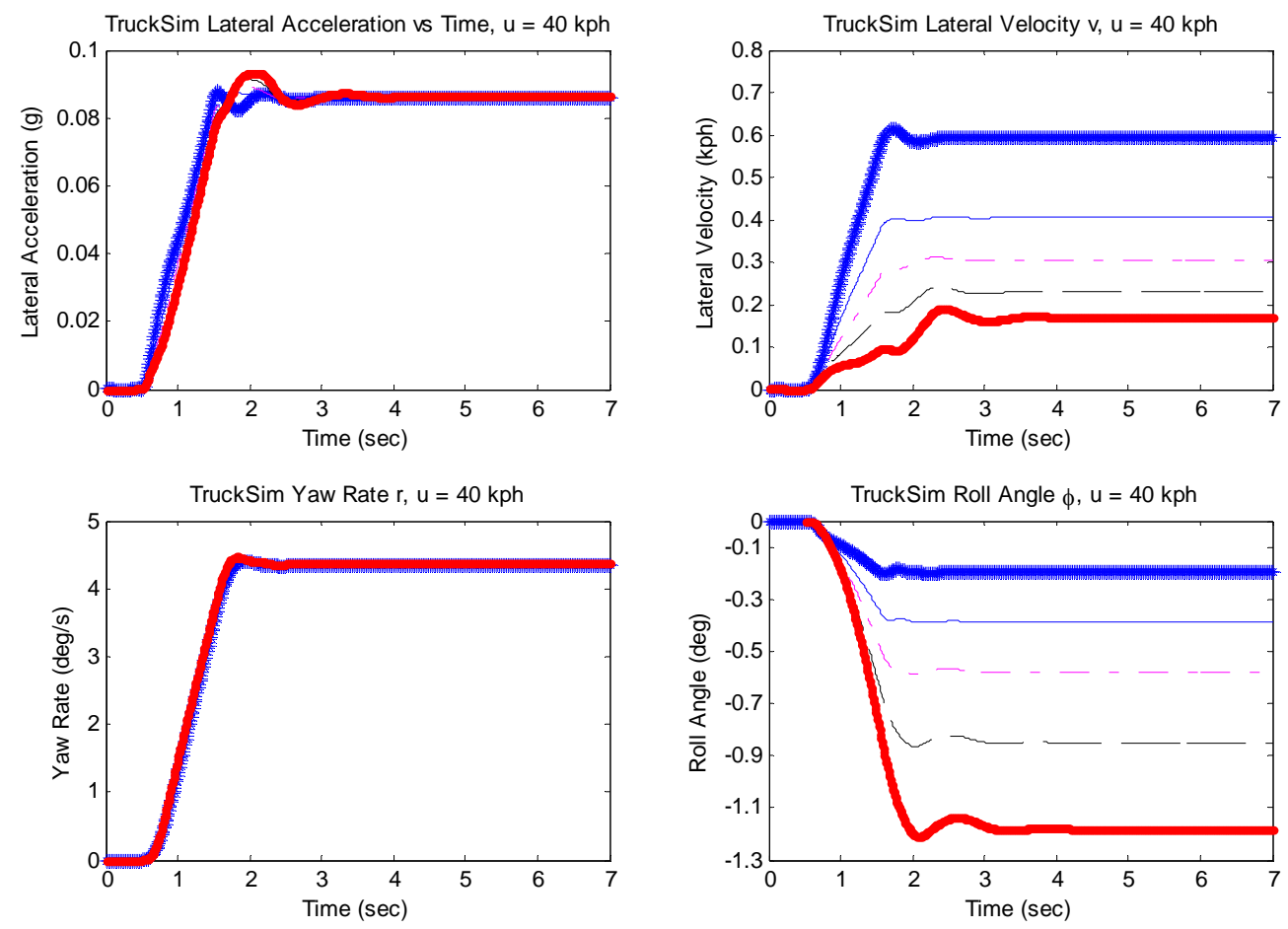

Figure 5.11 : TruckSim multimass vehicle study at $\mathrm{u}=40 \mathrm{kph}$ 

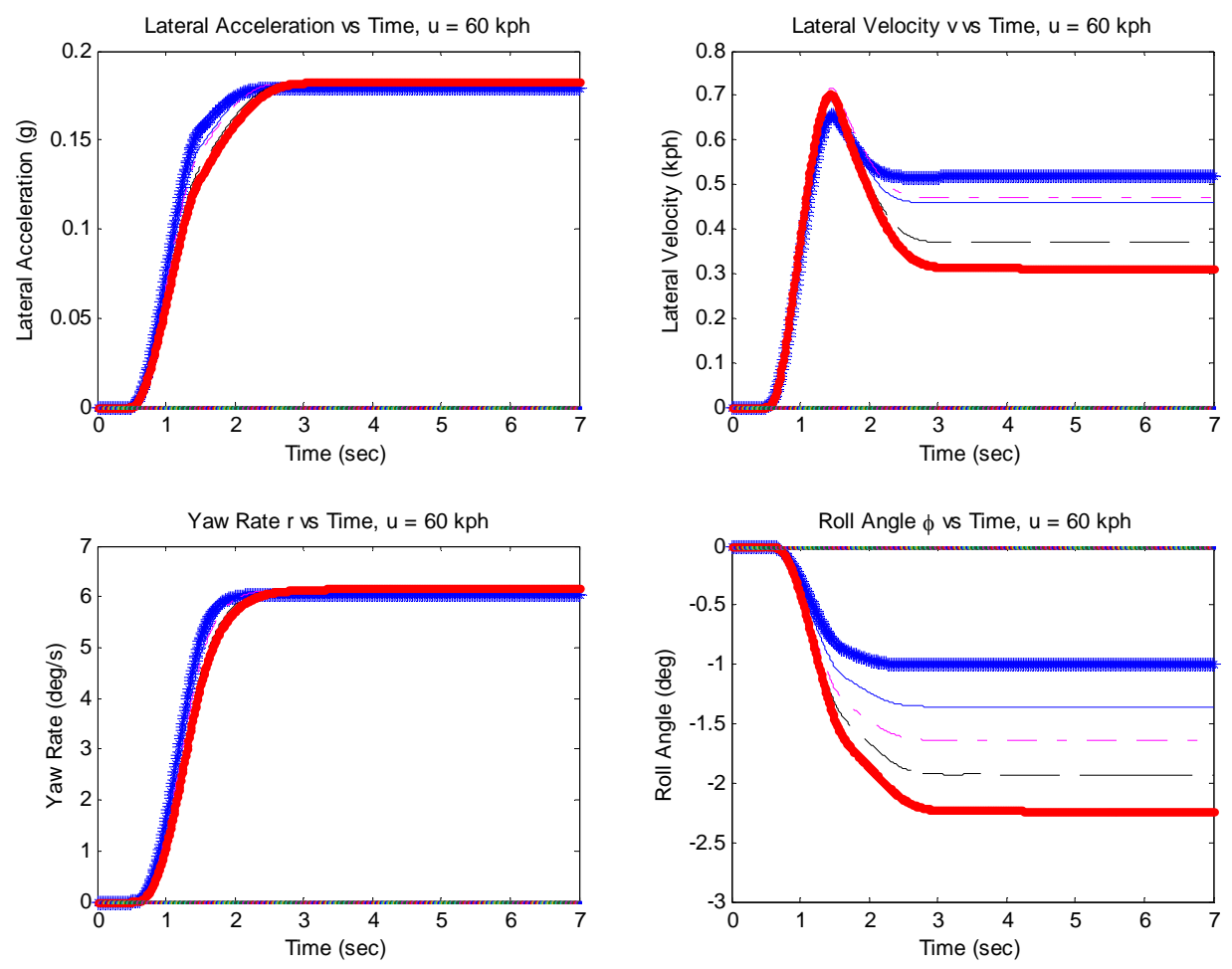

Figure 5.12 : 3DOF multimass vehicle study at $\mathrm{u}=60 \mathrm{kph}$
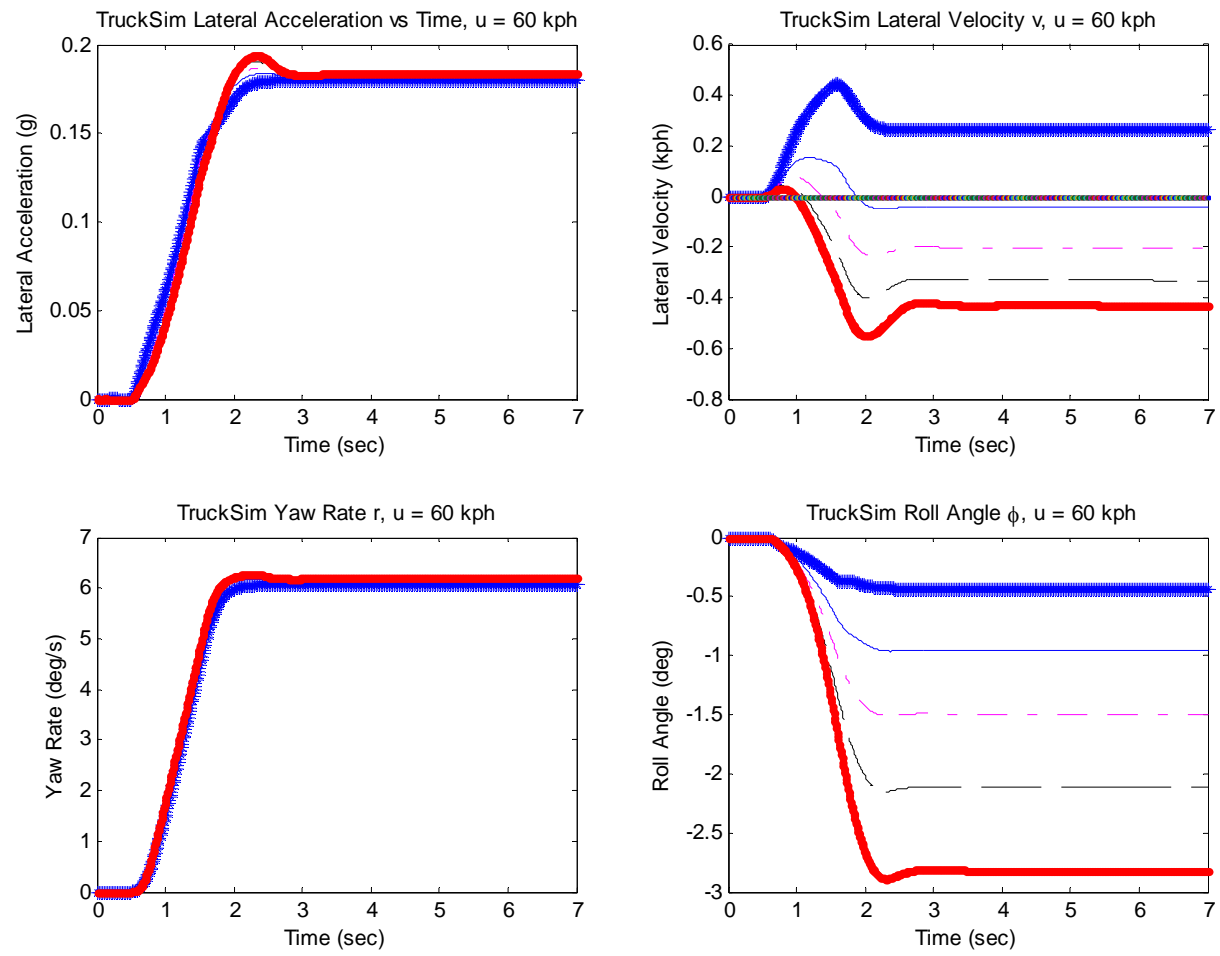

Figure 5.13 : TruckSim multimass vehicle study at $\mathrm{u}=60 \mathrm{kph}$ 

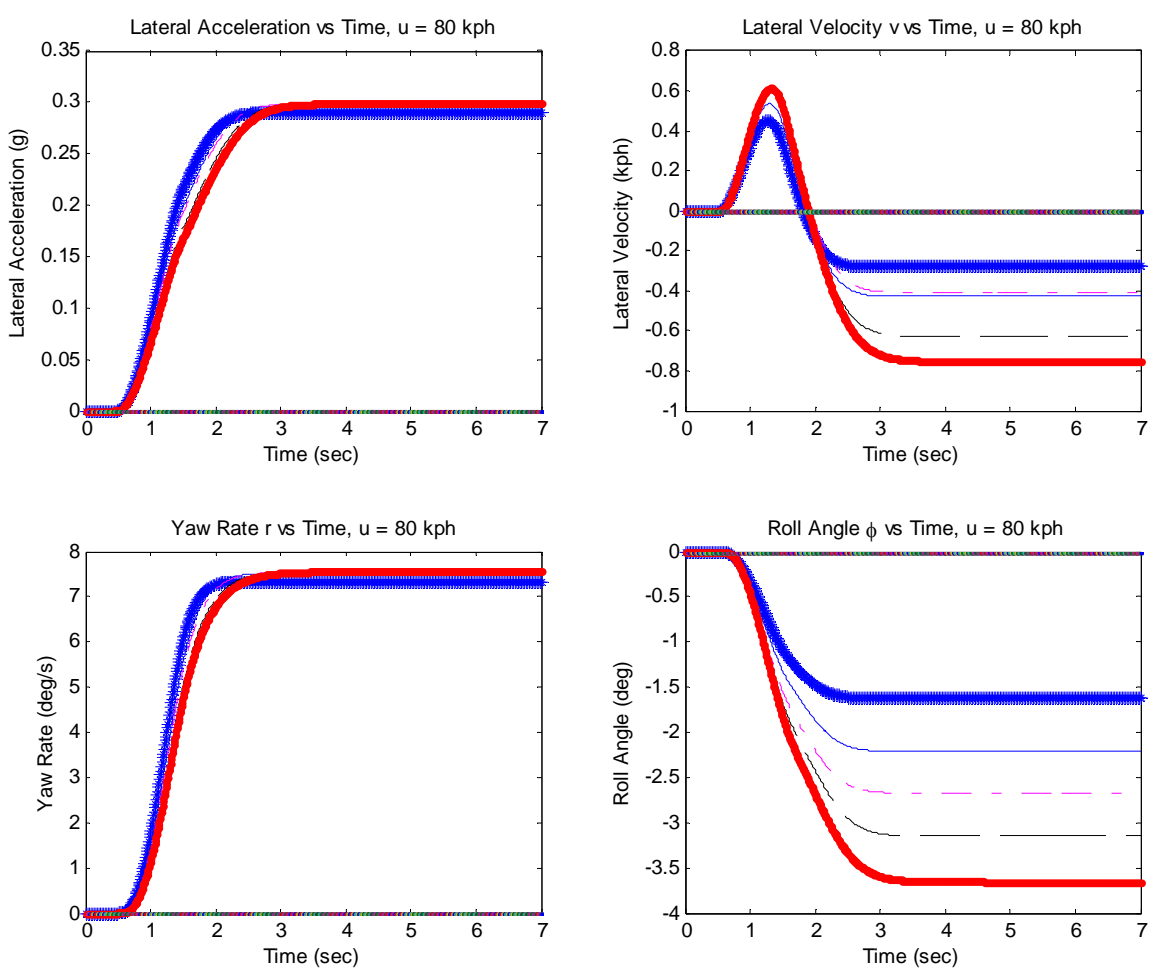

Figure 5.14 : 3DOF multimass vehicle study at $\mathrm{u}=80 \mathrm{kph}$
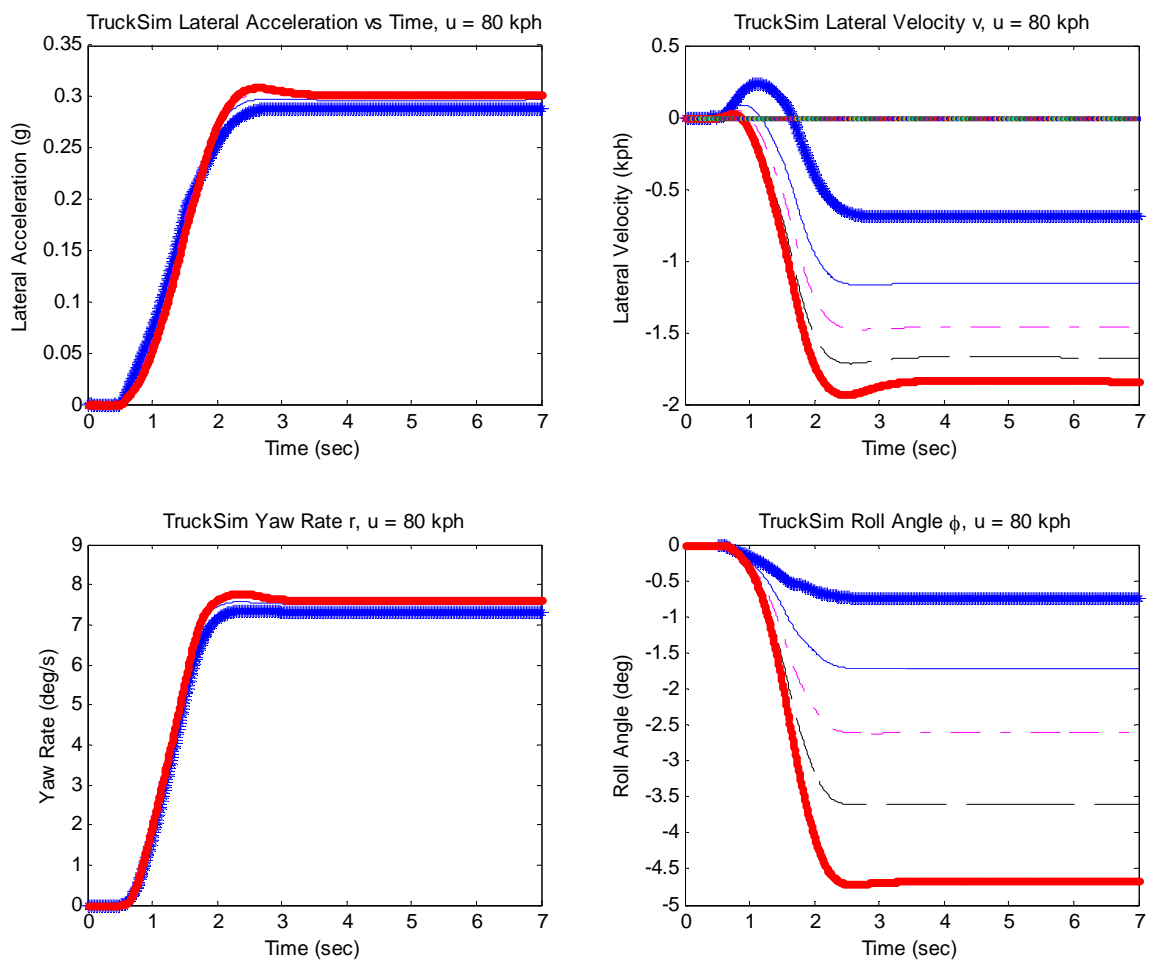

Figure 5.15 : TruckSim multimass vehicle study at $\mathrm{u}=80 \mathrm{kph}$ 


\subsection{Application of Fuzzy Logic}

Introduced in 1965 by American professor Lotfi Zadeh in his paper "Fuzzy Sets", fuzzy logic deals with reasoning that is approximate rather than precisely deduced from classical logic. It is a way of representing real world models in a format that is linguistic in nature. A fuzzy system can help model or control a system when a math model is not available that describes how the system's outputs depend on its inputs. The fuzzy system uses commonsense rules in place of the math model, building a bridge from the input space to the output space [16, 20-22]. In the case of vehicle control, if a driver is traveling down a road and realizes he is going too fast for the upcoming turn, he would presumably react to slow the vehicle down. The goal is to apply fuzzy logic in an intuitive fashion to the vehicle responses being measured. The process used to develop the fuzzy logic-based stability index, the Total Safety Margin (TSM), will then be discussed. For a brief background on fuzzy logic, see Appendix C.

\subsection{Membership Function Selection}

One of the more difficult areas of fuzzy logic is the choice of the membership function. In the most basic terms [22], a membership function assigns to each element $x$ of a given variable a certain value from the interval $[0,1]$ :

$$
\mu_{A}(x): X \rightarrow[0,1], \forall x \in X
$$

The assignment of each element $x$ of a given variable over the interval depends on the type of membership function used. For example, if a right sigmoid membership function is used then when the variable in question, lateral acceleration for example, is equal to zero, the membership grade is equal to zero. When the lateral acceleration is at its maximum, the membership grade is equal to 1. See Figure 5.16.

Membership functions can have many different forms, including triangular, trapezoidal, Gaussian, and left and right Sigmoids [16-22]. Since this thesis topic is interested in determining the instability levels of a vehicle, the membership function needed to be non-repeating over its defined interval. Specifically, any membership function that was symmetrical over the $x$ axis would yield the same membership grade for two response values. For example, if a symmetrical membership function such as a triangular, trapezoidal, or Gaussian were used for lateral acceleration, then depending on the specific shape of the membership function, a membership value of 0.5 could be possible for lateral accelerations of $0.2 \mathrm{~g}$ and $0.8 \mathrm{~g}$. This would imply that the vehicle was just as unstable at $0.2 \mathrm{~g}$ as it was at $0.8 \mathrm{~g}$. This would not make sense; therefore, an asymmetrical membership function had to be used.

The membership function also could not posses any abrupt changes in slope or discontinuities as is the case with triangular and trapezoidal membership functions. As Piegat points out, the main disadvantage of polygonal membership functions are that they are not continuously differentiable [22]. Therefore, a right sigmoid membership function was chosen because:

(1) They facilitate smooth, continuously differentiable surfaces of the fuzzy model. 
(2) They facilitate theoretical analysis of fuzzy systems as they are continuously differentiable and infinitely differentiable; i.e. they have derivatives of any grade [22].

A right sigmoid membership function was created for each of the four vehicle responses being studied in this thesis: Lateral Acceleration, Lateral Velocity, Yaw Rate, and Roll Angle. They are shown collectively in Figure 5.16.
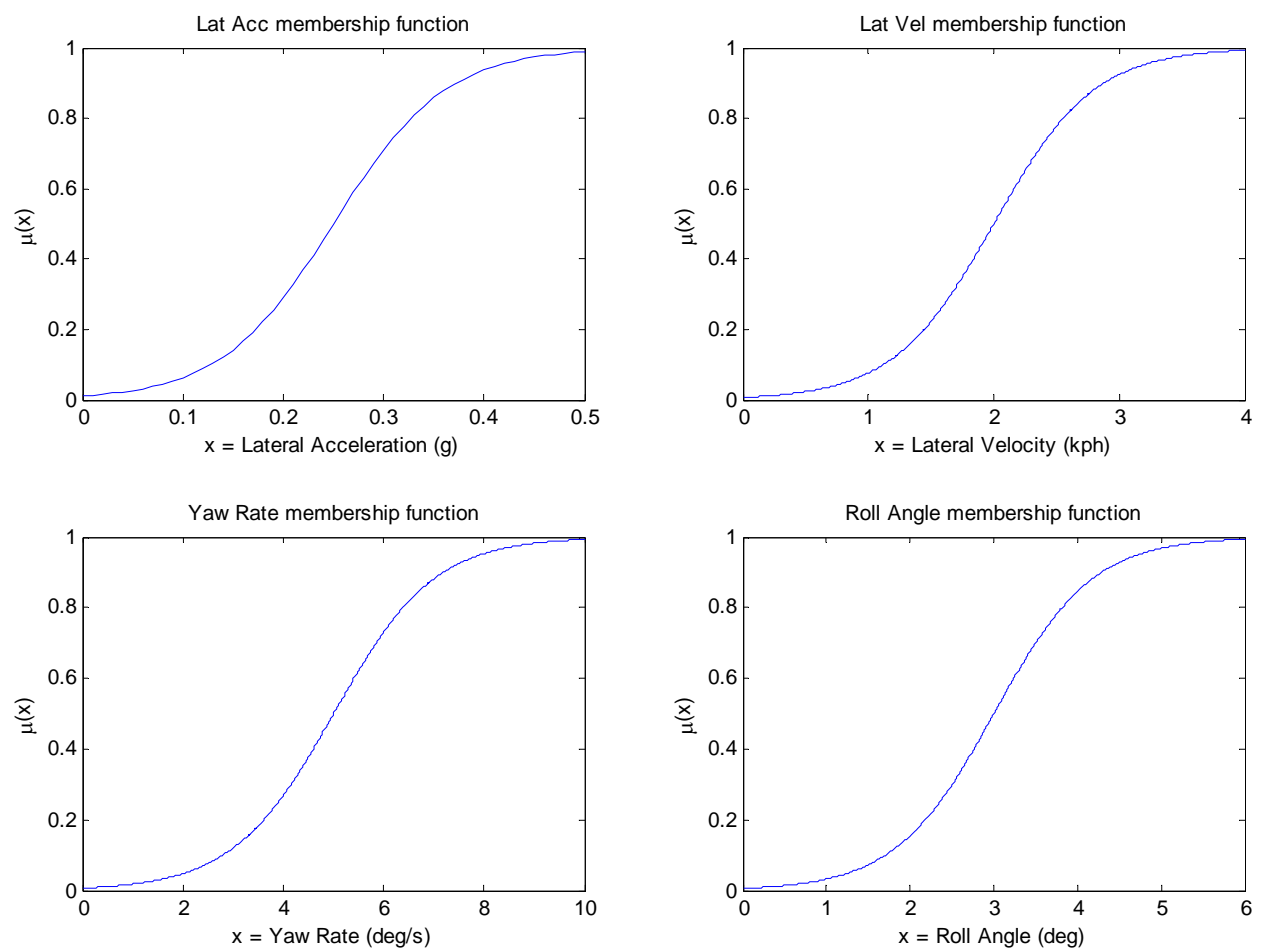

Figure 5.16 : Right Sigmoid membership functions

The equation for this function is [22]:

$$
\mu(x)=\frac{1}{1+\exp [-a(x-b)]}
$$

where:

$\mu(x)=$ Fuzzified value. Determines the degree of set membership. In this thesis this value is the calculated safety margin lm, vm, rm, and pm (Lateral Acceleration, Lateral Velocity, Yaw Rate, and Roll Angle, respectively) and is obtained by setting $x$ equal to the maximum value of Lateral Acceleration, Lateral Velocity, Yaw Rate, and Roll Angle.

$a=$ Slope factor. Increasing this value makes the sigmoid more vertical so with high values of $a$ the sigmoid can approach a step function. The slope factor $a$ can be calculated by estimating the 
value of $x_{0.99}$, i.e. the least value of the variable $x$ which can be admitted with almost complete certainty to belong to a fuzzy set represented by the sigmoid function.

$b=$ Determines the characteristic coordinate of the point $k$, at which the grade of membership in the fuzzy set amounts to 0.5, i.e. at $x=b, \mu(x)=0.5$.

$x=$ The $\mathrm{x}$-axis of the membership function. In our case these are the vehicle response ranges of Lateral Acceleration, Lateral Velocity, Yaw Rate, and Roll Angle.

The membership $\mu(x)$ represents the vehicle instability level for each variable: Lateral Acceleration, Lateral Velocity, Yaw Rate, and Roll Angle. These are referred to as the safety margins and represented as: Lateral Acceleration (lm), Lateral Velocity (vm), Yaw Rate (rm), and Roll Angle (pm). When these vehicle responses were all zero, the vehicle was stable and therefore the memberships were all zero. When these vehicle responses were at their maximum and therefore represented an unstable situation for the given region, the memberships were all equal to one. Based on this convention the following fuzzy rules were established:

If membership $\mu(x)$ of output $=0$ then Safe

If membership $\mu(x)$ of output $=1$ then Danger

The next step was to determine the proper vehicle response ranges for these membership functions. Since the determination of the vehicle's instability level was of interest, it was necessary to determine the test vehicle's range of dynamic capabilities in the chosen maneuver. Since real vehicle data was not available TruckSim was used for this testing.

The vehicle was loaded to the same test loads as in the multimass study but this time each vehicle mass was run through the chosen maneuver until the inside tire lifted off the ground within the first seven seconds but touched back down by 10 seconds. The seven second time was chosen since in the multimass studies the vehicle had attained steady state values within four seconds and the total simulation time was seven seconds. Touchdown by 10 seconds was chosen since the goal was not to test for vehicle rollover, but rather when the vehicle would just lift its inside tires. Wheel lift was checked for during the animation and confirmed on the vertical tire load plots available within TruckSim. These speeds at which the inside wheel briefly lifted and touched back down indicated a maximum safe cornering speed. The TruckSim simulation time of 20 seconds was chosen to ensure that the vehicle didn't rollover at any of these speeds.

The results of these tests are shown in Figures 5.17 - 5.19 and Table 5.11. 


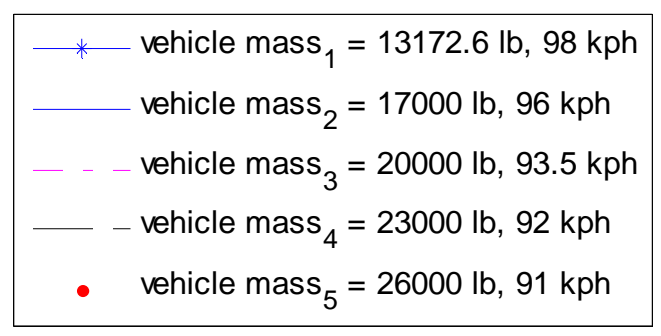

Figure 5.17 : Legend for TruckSim Wheel Lift Study

\begin{tabular}{|cr|}
\hline \multicolumn{2}{|c|}{ Speed Comparison Chart } \\
\hline $98 \mathrm{kph}=$ & $60.89 \mathrm{mph}$ \\
$96 \mathrm{kph}=$ & $59.65 \mathrm{mph}$ \\
$93.5 \mathrm{kph}=$ & $58.10 \mathrm{mph}$ \\
$92 \mathrm{kph}=$ & $57.17 \mathrm{mph}$ \\
$91 \mathrm{kph}=$ & $56.54 \mathrm{mph}$ \\
\hline
\end{tabular}

Figure 5.18 : Speed Comparison Chart for TruckSim 7 Wheel Lift Speeds

As can be seen from Figure 5.18, there is only a $7 \mathrm{kph}(4.35 \mathrm{mph})$ difference between the unladen and heaviest loaded vehicles for limit cornering speeds in this test.
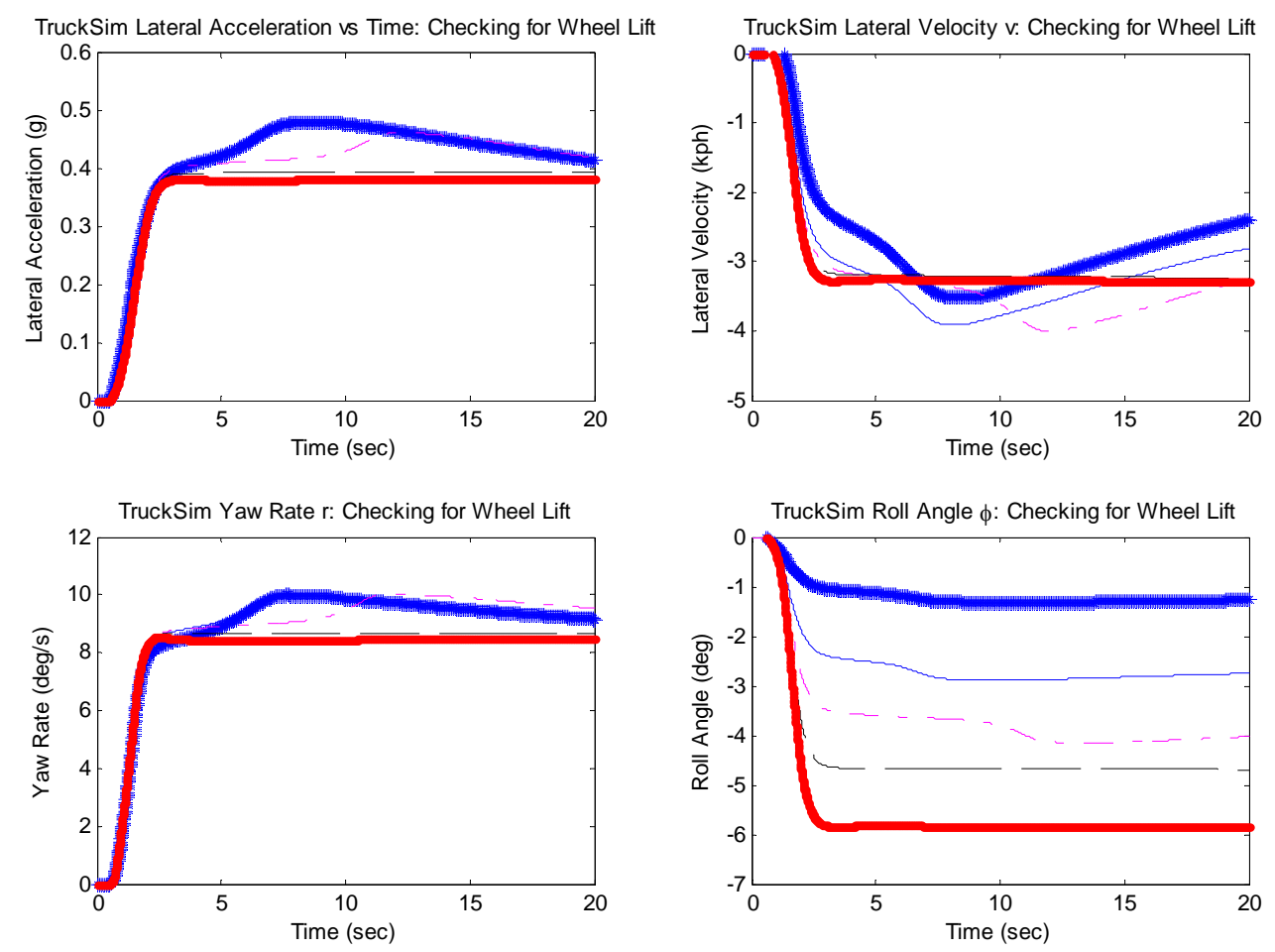

Figure 5.19 : TruckSim Wheel Lift Study 
Looking at Figure 5.19, it can be seen that the upper limits for each of the vehicle responses are:

\begin{tabular}{|l|c|l|}
\hline \multicolumn{3}{|c|}{ Wheel Lift Study $^{\mid}$} \\
\hline Lateral Accleration $_{\max }$ & 0.5 & $\mathrm{~g}$ \\
Lateral Velocity $_{\max }$ & 4 & $\mathrm{kph}$ \\
Yaw Rate $_{\max }$ & 10 & $\mathrm{deg} / \mathrm{s}$ \\
Roll Angle $_{\max }$ & 6 & $\mathrm{deg}$ \\
\hline
\end{tabular}

Table 5.11 : Wheel Lift Study Vehicle Response Thresholds

Therefore these limits were chosen as the upper limits on the intervals for the membership functions. Note that the magnitudes of the simulated values are shown and were used in the membership function calculations.

To put these results in perspective, a common measure of a vehicle's static stability is the static stability factor [37, 39], where for a simplified, quasi-static model:

$$
S S F=\frac{t}{2 h}
$$

where:

SSF $=$ static stability factor

$\mathrm{t}=$ vehicle track width

$\mathrm{h}=$ vehicle CG height above the road

Equation 5.3 represents a simplified metric to estimate the maximum cornering limit of a vehicle. It represents the maximum sustainable lateral acceleration of a vehicle in a steady state turn.

For the unladen TruckSim test vehicle and using the rear track width the SSF for this vehicle is:

$$
\begin{aligned}
& S S F=\frac{t}{2 h}=\frac{1.829 m}{2 * 1.173 m}=0.7796 \\
& S S F=0.78
\end{aligned}
$$

Based on the results of [37, 39], an SSF for a pick-up trucks, SUVs, and vans have SSF values in the range of 1.0 to 1.3. Thus, and SSF of 0.78 for a vehicle of the type simulated here is reasonable and indicates a higher propensity of static rollover than a typical passenger vehicle. It should be noted that including the tire and suspension stiffnesses result in a lower stability factor than the SSF calculation. 


\subsection{Weighting Factor Determination}

Defuzzification is the process by which a single numerical output value is determined from the summation of the grades of membership as determined by the membership functions. This operation is accomplished by a defuzzification mechanism such as [16-22]:

- Center of Area

- Center of Gravity

- Center of Maxima

- Picking the mode or maximum value of the output set

- Weighted average

The method of defuzzification used here is called the weighted average method and has been applied successfully in both vehicle control and non-vehicle applications, specifically [17] and [19]. The Total Safety Margin, as described in the next section, has the form of a weighted average.

In the defuzzification process rule weights can be used in the sum to scale each term to reflect rule credibility [16-22, 38], or in this case the importance of each dynamic response specific to the vehicle type being modeled. To determine the weighting factors it was necessary to understand the test vehicle's range of dynamic capabilities in the maneuver chosen, as was explained above in Section 5.2. Since these results represented the upper limit of the vehicle's capabilities we wanted to use these as the basis for comparison to the simulation results obtained in Section 5.1. The vehicle was closest to its upper limits in the $80 \mathrm{kph}$ case, this speed representing a reasonably safe cornering speed. Specifically, by comparing the maximum values of the TruckSim results from this speed event to those obtained in the lifted wheel cases, we can obtain dimensionless weighting factors representative of each of the dynamic responses tested. For example, in the case of Lateral Acceleration the $80 \mathrm{kph}$ maximum value was $0.3092 \mathrm{~g}$ and the lifted wheel maximum value was $0.4810 \mathrm{~g}$. Therefore $0.3092 \mathrm{~g} / 0.4810 \mathrm{~g}=0.6428$, meaning that at $80 \mathrm{kph}$ the Lateral Acceleration is at $64.28 \%$ of its maximum value. Table 5.12 summarizes these results for all of the vehicle responses. A multiplication factor of 10 was used to scale the weighting factors between zero and 10 . 


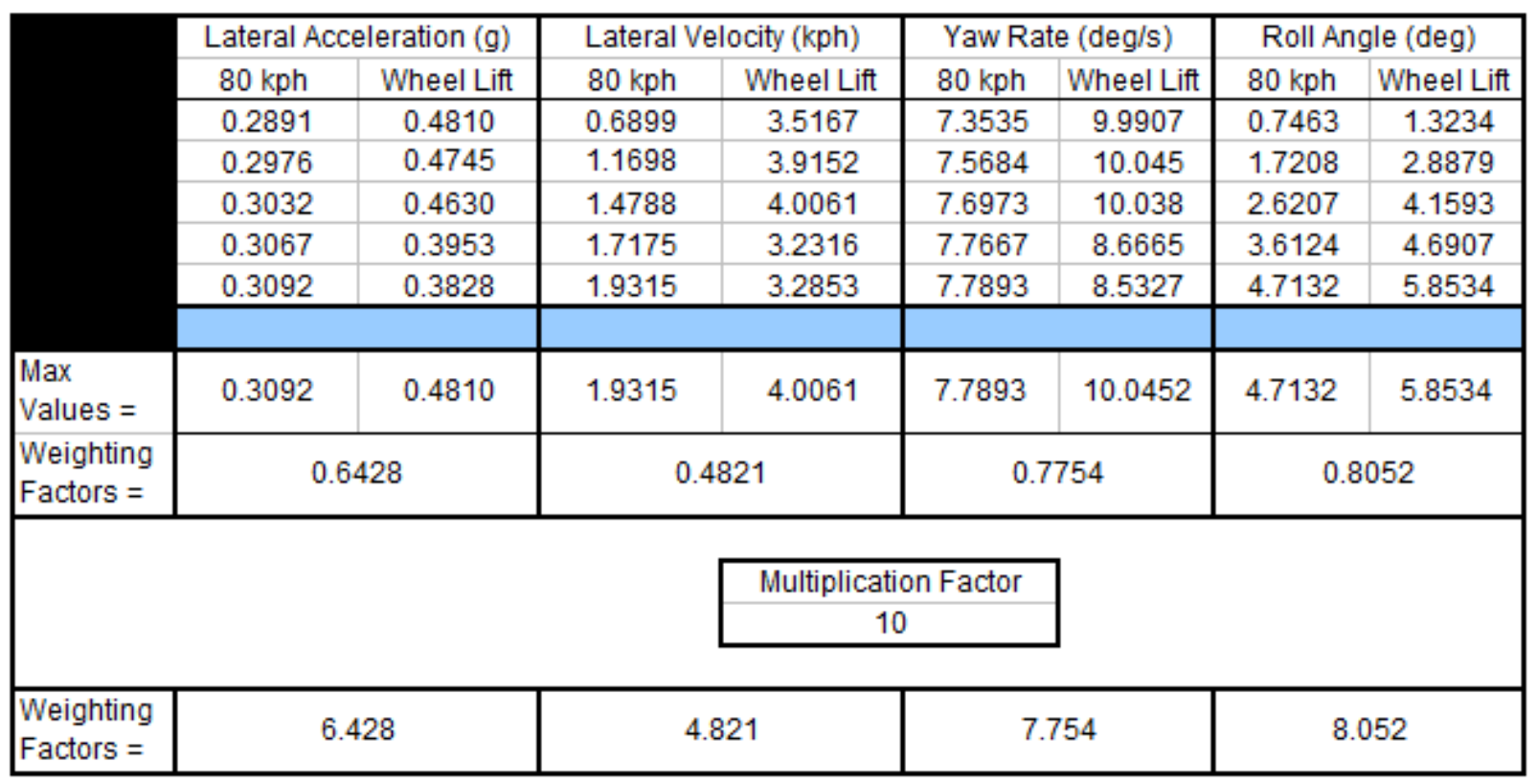

Table 5.12: Safety Margin Weighting Factors

\subsection{Fuzzy Logic-Based Total Safety Margin (TSM) with GPS Input}

As described previously, the Total Safety Margin (TSM) was fuzzy logic-based since fuzzy logic is an accepted technique to deal with vehicle control and it is also a powerful way to represent human knowledge in control without the need to develop extensive mathematical equations and complex real world models [1]. Since the driver would presumably slow the vehicle down himself if he knew he was going too fast for the turn, a fuzzy logic-based control makes sense. As described in the assumptions at the beginning of this chapter, the job of the GPS-assist is to detect the curve in advance and provide the road characteristics data to the on-board GPS Controller which would then decide the instability level of the vehicle using the Total Safety Margin, or TSM. See Figure 5.21.

The safety margins are used in conjunction with the weighting factors shown in Table 5.13 to determine the Total Safety Margin (TSM), defined as:

$$
T S M=\frac{l m * W_{l m}+v m * W_{v m}+r m * W_{r m}+p m * W_{p m}}{\left(W_{l m}+W_{v m}+W_{r m}+W_{p m}\right)}
$$




\begin{tabular}{|c|c|c|c|}
\hline \multicolumn{4}{|c|}{ TSM Matrix - Matlab 3 DOF } \\
\hline & $40 \mathrm{kph}$ & $60 \mathrm{kph}$ & $80 \mathrm{kph}$ \\
\hline 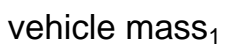 & 0.1224 & 0.2791 & 0.4504 \\
\hline vehicle mass $_{2}$ & 0.1249 & 0.2946 & 0.4976 \\
\hline vehicle mass $_{3}$ & 0.1266 & 0.3056 & 0.5462 \\
\hline vehicle mass $_{4}$ & 0.1276 & 0.3206 & 0.6071 \\
\hline 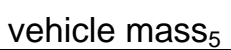 & 0.1297 & 0.3432 & 0.6657 \\
\hline
\end{tabular}

\begin{tabular}{|c|c|c|c|}
\hline \multicolumn{4}{|c|}{ TSM Matrix - TruckSim 7} \\
\hline & $40 \mathrm{kph}$ & $60 \mathrm{kph}$ & $80 \mathrm{kph}$ \\
\hline vehicle mass $_{1}$ & 0.1217 & 0.2729 & 0.4291 \\
\hline vehicle mass $_{2}$ & 0.1231 & 0.2851 & 0.4648 \\
\hline vehicle mass $_{3}$ & 0.1260 & 0.3020 & 0.5440 \\
\hline vehicle mass $_{4}$ & 0.1292 & 0.3420 & 0.6656 \\
\hline vehicle mass $_{5}$ & 0.1347 & 0.4234 & 0.7302 \\
\hline
\end{tabular}

Table 5.13 : TSM Matrices for 3DOF Matlab Simulation and TruckSim

\begin{tabular}{|c|c|c|c|c|c|c|}
\hline & \multicolumn{6}{|c|}{ TSM Matrix - Percent Error and Difference } \\
\hline & \multicolumn{2}{|c|}{$40 \mathrm{kph}$} & \multicolumn{2}{|c|}{$60 \mathrm{kph}$} & \multicolumn{2}{|c|}{$80 \mathrm{kph}$} \\
\hline & $\%$ Error & $\begin{array}{l}\text { Difference }= \\
\text { TS7 - Matlab }\end{array}$ & $\%$ Error & $\begin{array}{l}\text { Difference }= \\
\text { TS7 - Matlab }\end{array}$ & $\%$ Error & $\begin{array}{l}\text { Difference = } \\
\text { TS7 - Matlab }\end{array}$ \\
\hline vehicle mass $s_{1}$ & 0.58 & -0.0007 & 2.27 & -0.0062 & 4.96 & -0.0213 \\
\hline vehicle mass $_{2}$ & 1.46 & -0.0018 & 3.33 & -0.0095 & 7.06 & -0.0328 \\
\hline vehicle mass $_{3}$ & 0.48 & -0.0006 & 1.19 & -0.0036 & 0.40 & -0.0022 \\
\hline vehicle mass $_{4}$ & 1.24 & 0.0016 & 6.26 & 0.0214 & 8.79 & 0.0585 \\
\hline vehicle mass $_{5}$ & 3.71 & 0.0050 & 18.94 & 0.0802 & 8.83 & 0.0645 \\
\hline
\end{tabular}

Table 5.14 : TSM Matrix - Percent Error and Difference

As can be seen from the TSM Matrices shown in Tables 5.13 - 5.14, the 3DOF simulation results were very close to the TruckSim results. The lowest percent error of $0.58 \%$ was for vehicle mass $_{1}$ at $40 \mathrm{kph}$. The largest percent error of $18.94 \%$ was for vehicle mass ${ }_{5}$ at $60 \mathrm{kph}$. This means that for the $0.58 \%$ error, the Matlab model predicted a TSM 0.0007 larger than TruckSim. In this case, the Matlab model is predicting a slightly higher level of instability than TruckSim. For the $18.94 \%$ error, the Matlab model predicted at TSM 0.0802 less than TruckSim. In this case, the Matlab model was under-predicting the instability level of the vehicle compared to TruckSim. 
Since the TSM values are direct results of the results obtained from the multimass studies, further investigation would need to be performed to see how significant these differences are and to what extend would these influence a stability control system.

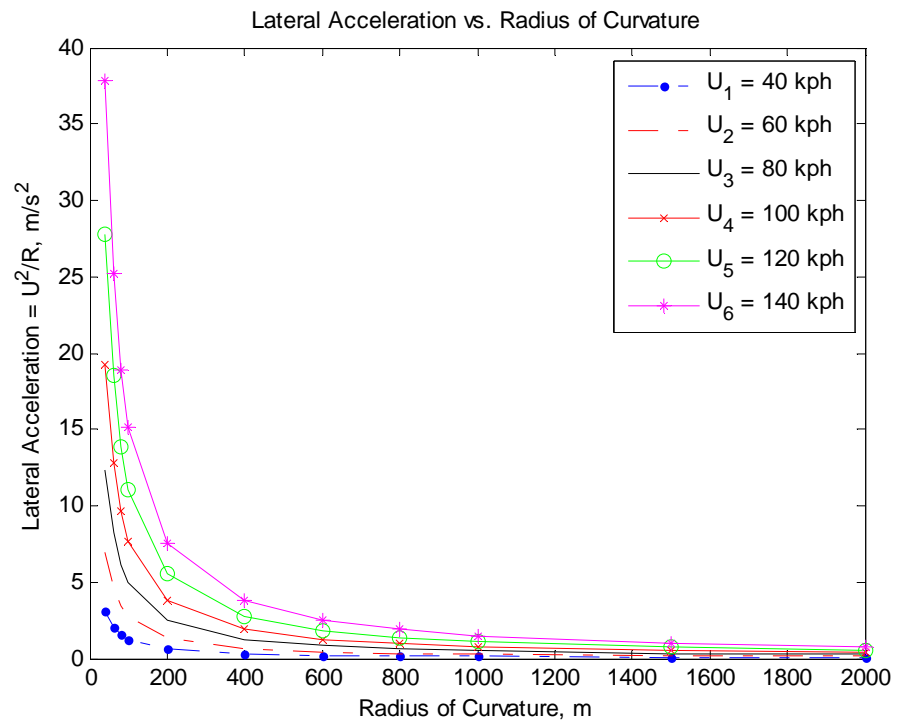

Figure 5.20: Lateral Acceleration vs. Radius of Curvature assuming uniform circular motion

Figure 5.20 shows the steady state lateral acceleration as a function of road radius. When the vehicle is traveling on a straight, level road the radius of curvature is very large and so the lateral acceleration, lateral velocity, yaw rate, and roll angle would be zero or nearly zero. Therefore all of the safety margins would be zero and the TSM would decide the vehicle condition was "Safe". Alternatively the radii of curvature could decrease to a point such that the aforementioned safety margins resulted in a TSM of one which would indicate a "Danger" condition.

\subsection{Vehicle Simulation Conclusions}

A new fuzzy logic-based predictive control method was developed which is capable of predicting the vehicle instability level through the use of GPS data and predictive vehicle modeling. The simulation results for five vehicle masses at three longitudinal velocities in both Matlab and TruckSim, as well as the five additional data points for the wheel lift study, showed the applicability of the methodology for heavy trucks where the output of the fuzzy controller was used as inputs to the Total Safety Margin, or TSM in the form of the safety margins lm, vm, rm, and pm (Lateral Acceleration, Lateral Velocity, Yaw Rate, and Roll Angle, respectively). Rule weights, determined by comparing the TruckSim $80 \mathrm{kph}$ and Wheel Lift Study results, were used in the TSM to scale each term to reflect rule credibility, or in this case the importance of each dynamic response specific to the vehicle type being modeled. Using these rule weights the TSM Matrices for both the 3DOF and TruckSim simulations were presented. These matrices showed similar results and indicated that the 3DOF model is capable of predicting the vehicle instability level close to TruckSim. 


\subsection{Vehicle Simulation Future Work}

As described previously, this system works by taking the GPS signal (i.e. radius of curvature and other roadway properties) and providing the data to the Predictive Dynamics Model which finds Lateral Velocity, Yaw Rate, Roll Angle, and Roll Rate. The Lateral Acceleration is then calculated. See Figure 5.21 below.

As stated above, further investigation needs to concentrate on understanding the lateral velocity plots. Also, a more detailed tire model such as Pacejka or Dugoff needs to be incorporated into the Matlab simulation to confirm the validity of the linear tire assumption. A two track model would also be a worthwhile comparison, specifically to include and better model the lateral load transfer mechanism. Finally, the TSM would need to be run in-the-loop with TruckSim to see how well it can predict vehicle instability.

One significant goal will be to incorporate the hardware-in-the-loop (HIL) simulator, discussed below in Chapter 6, with the TSM and TruckSim RT/CarSim RT and add an electronic stability control model. This would extend the TSM functionality. Additional methods for a stability index could include an adaptive fuzzy methodology. In that instance, the TSM matrices could serve as look-up tables in a stability control system based on simulation and test data. This system would work such that when the vehicle begins its drive cycle, the TSM would assume a worst case scenario in terms of loading and vehicle speed. As the vehicle drives and vehicle response data is collected: lateral acceleration, lateral velocity, yaw rate, and roll angle, the TSM would be recalculated in real-time. These new TSM values would be compared to the TSM matrices stored on-board, and if necessary an interpolation routine performed to determine the current vehicle instability index. This would be especially valuable for a cargo truck like the one simulated in this thesis since the loading conditions would change throughout the day. As a result, the TSM would also change. To complete the TSM incorporation into the vehicle, a driver warning system such as the type presented in [23, 35] could be investigated and implemented.

The Total Safety Margin (TSM) would be an input into a Decision Making block (See Figure 5.21). This would determine the required vehicle stabilizing actions based on the overall TSM results. This overall vehicle safety condition would be used along with the onboard EBS (electronic brake system) sensors as inputs to the EBS controller which would send the necessary signals to the brake control actuators. These would command and then apply the necessary vehicle stabilizing brake pressures and if need be, reduce engine torque to keep the vehicle within the specified safety regions, thus remaining under control.

This information would be run through an Actual Plant Dynamics block which would contain all of the vehicle sensor information and as such would have collected information on the stabilized vehicle, i.e., has the vehicle stabilized or not. This information would feed back into the Predictive Dynamics Model as the new Initial Conditions along with updated GPS data and the cycle would repeat. 


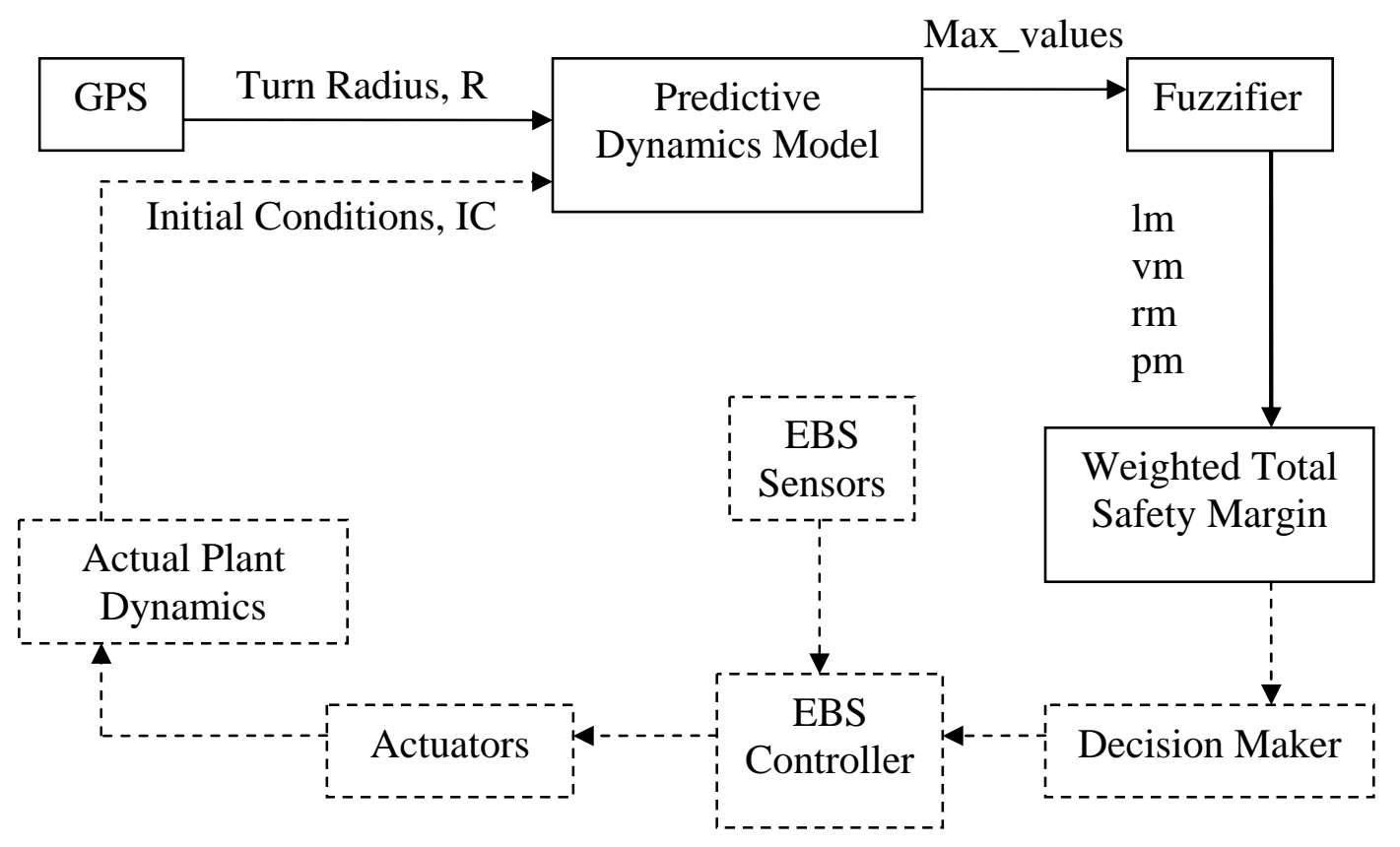

Figure 5.21: GPS State-Flow Diagram

The dashed portions shown in Figure 5.21 represent future work to be done on this controller design. 


\section{Chapter 6}

\section{Hardware-in-the-Loop (HIL) Simulation : Introduction and Technical Overview}

An HIL, or Hardware-in-the-Loop, simulator is a laboratory test environment which combines computer simulation with embedded hardware. HIL simulators for automotive applications are designed to test steering, braking, vehicle dynamics, or testing of an automotive ECU (electronic control unit) itself. For example, Velardocchia and Sorniotti document the necessary tests to characterize, on the bench, a commercially available electronic stability control (ESC) hydraulic control unit (HCU) using the Politecnico di Torino Hardware-in-the-Loop brake systems test bench [25]. Their tests included studying the step responses of each valve as well as the pump motor. Using this HIL simulator, they go on to compare different commercial ABS strategies in a large spectrum of maneuvers, including slow and panic brake applications as well as split- $\mu$ and alternating- $\mu$ maneuvers [26]. Kohl and Jegminat describe the various test phases during the development of automotive electronics using HIL simulation [27] while Schuette and Waeltermann provide a technical survey of state-of-the-art HIL test systems for vehicle dynamics controllers. These controllers include ABS, TCS, and ESC functionality [28]. Segawa, Nakano, Shino, and Nagai conducted a preliminary study concerning the quantitative analysis of a passenger vehicle steering system using HIL simulation. The purpose of their study was to develop a steering HIL simulator that could quantitatively evaluate different steering systems. Critical to this evaluation was the force feedback to the steering wheel which was accomplished via a controllable force on the tie rod [29].

For the project described here, the intention was to build a driving simulator which, once completed, would be capable of running vehicle dynamics simulation programs such as Mechanical Simulation Corporation's CarSim RT ${ }^{\circledR}$ (real-time) and TruckSim RT ${ }^{\circledR}$ and allow the users to test their own brake control algorithms without the expense or requirement of testing at an automotive proving ground. In-vehicle testing, while necessary for vehicle manufacturers and their suppliers, can be expensive and test results can vary due to such factors as weather, excessive component wear, and driver variability. This simulator aims to eliminate many of these factors using a controlled laboratory environment while still allowing the driver to fully participate in the algorithm development.

Since the goal was to fully integrate the driver's inputs with the CarSim RT and TruckSim RT simulation programs and be able to collect the same set of data that would be available from an 
in-vehicle test with an instrumented vehicle, the HIL simulator was to include a steering wheel, accelerator and brake pedals, as well as a complete automotive hydraulic braking system from the brake booster to the rotors with ABS, TCS, and ESC functionality. After considering the timing requirements to accomplish this task, it was decided the order of operations would be (1) steering; (2) accelerator pedal; (3) braking system. The first task, steering the CarSim RT / TruckSim RT vehicle, is documented here.

\subsection{HIL Simulator Design: Main Platform}

The Virginia Tech Center for Vehicle Systems and Safety's (CVeSS) driving simulator was designed to be a simple, self-contained unit that is easily transportable, rugged and modular.

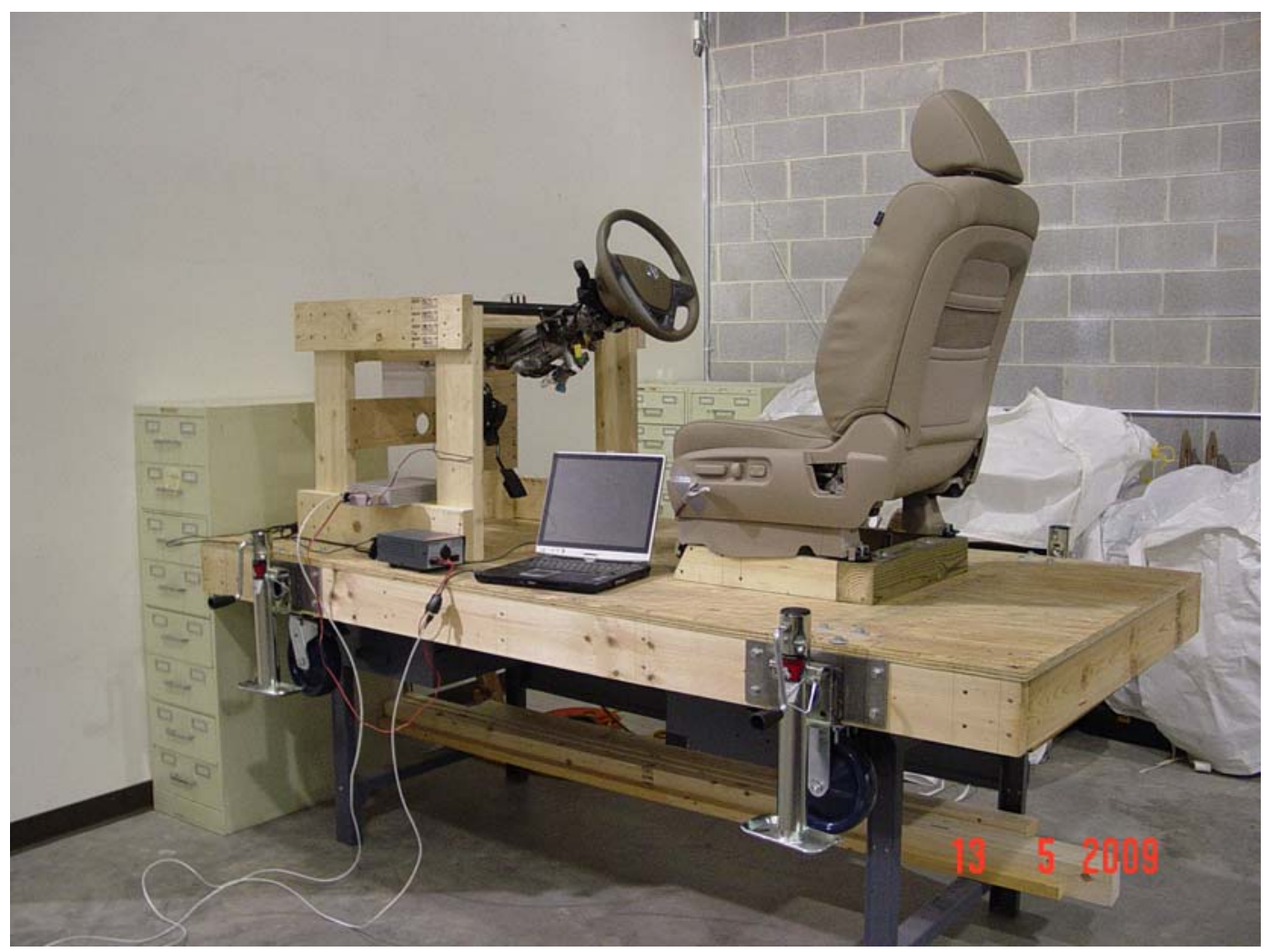

Figure 6.1: Left rear side view of CVeSS HIL driving simulator showing seat and steering module.

Constructed of commonly available materials the simulator, which is shown in the Figures 6.1 6.3, features lockable, swivel casters in front and non-swiveling casters in the rear with ball bearing-supported 8" diameter wheels at all four corners. These will allow the simulator to be rolled easily even with the addition of HIL equipment. This HIL simulator also contains integrated manual crank jacks mounted just to the outside of the casters to facilitate raising and lowering of the simulator and, in conjunction with the locking front casters, eliminate the possibility of rollaway while tests are being performed. Since the four jacks can be raised and 
lowered independently they also allow the simulator to remain level on uneven floor surfaces. The seat is electrically adjustable to accommodate a range of driver sizes.

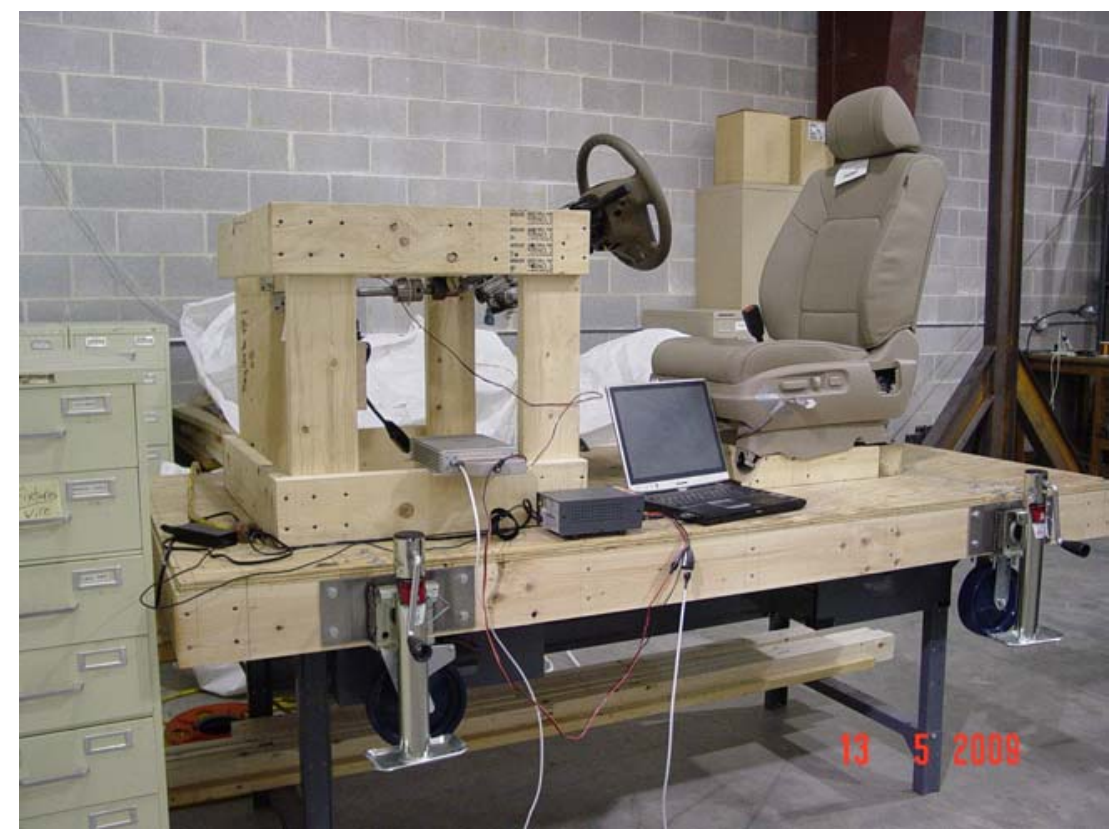

Figure 6.2: Left front side view of CVeSS HIL driving simulator

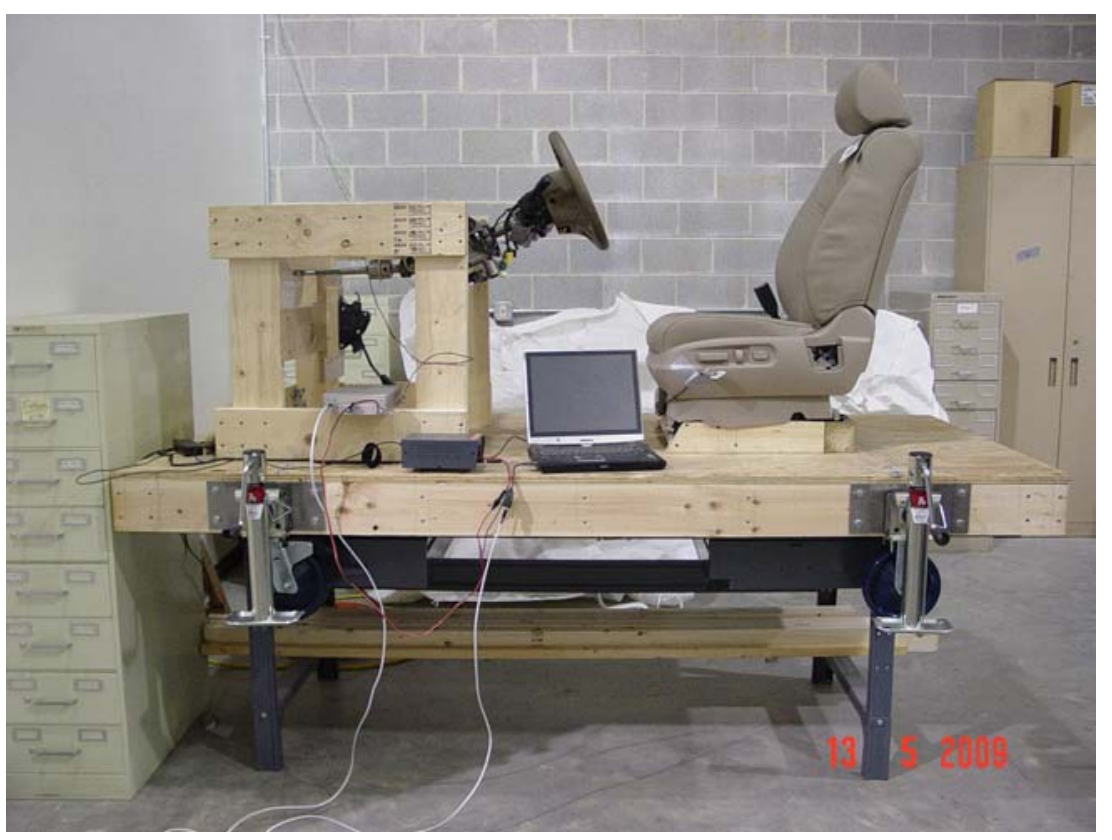

Figure 6.3: Left side view of CVeSS HIL driving simulator 


\subsection{Steering Module}

The steering module was designed to be easily transported and serve as a self-contained unit for the driver interfaces, specifically steering wheel and pedals. The unit can be seen in Figures $6.4-$ 6.6 .

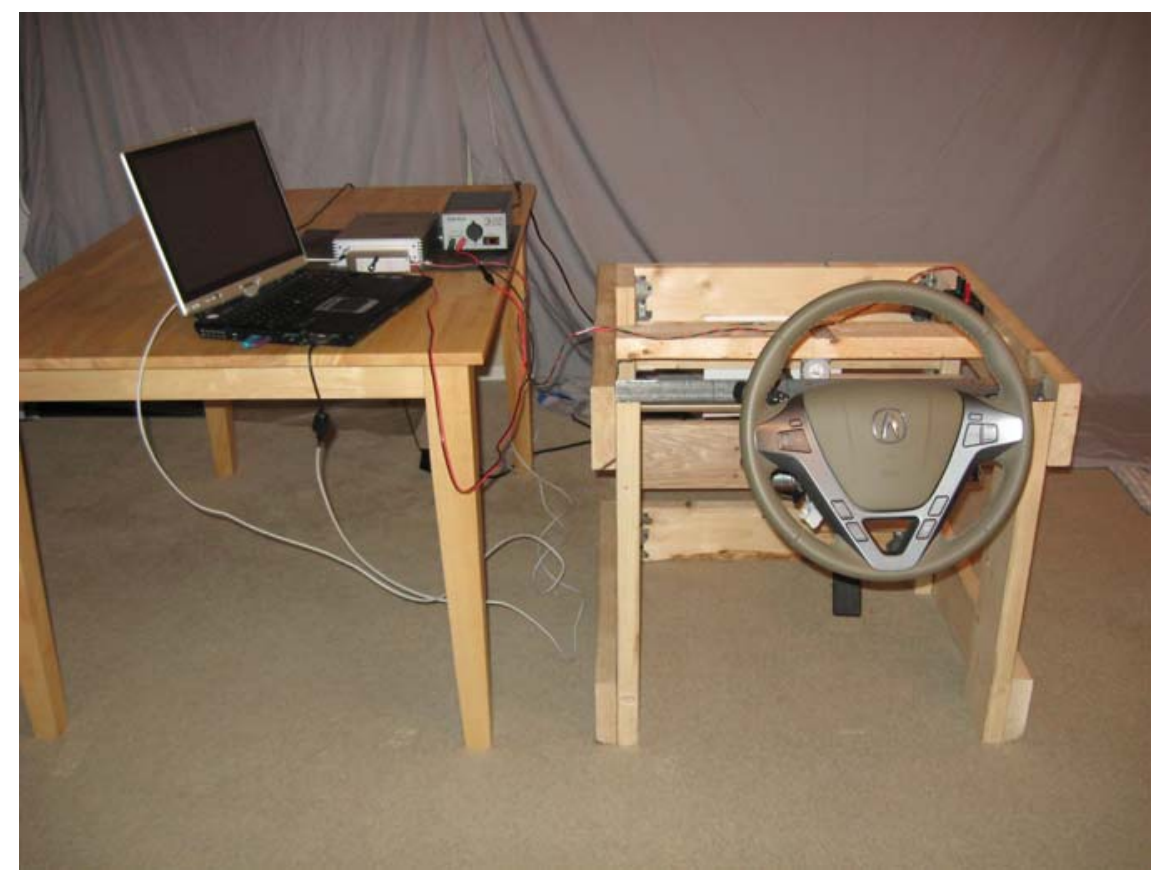

Figure 6.4 : Steering HIL simulator with support equipment

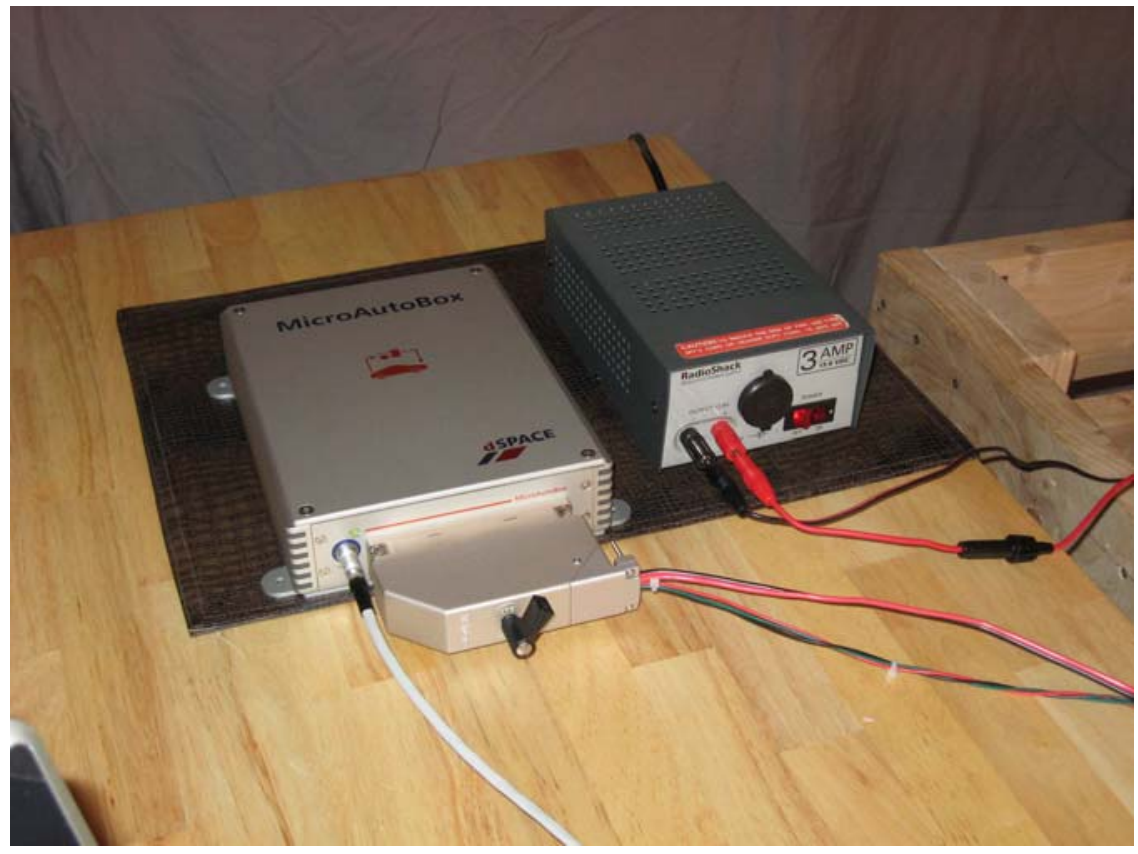

Figure 6.5. dSPACE MicroAutoBox 1401/1501 and power supply. Black cylindrical container on the positive (red) power supply wire contains a $10 \mathrm{amp}$ fuse. 


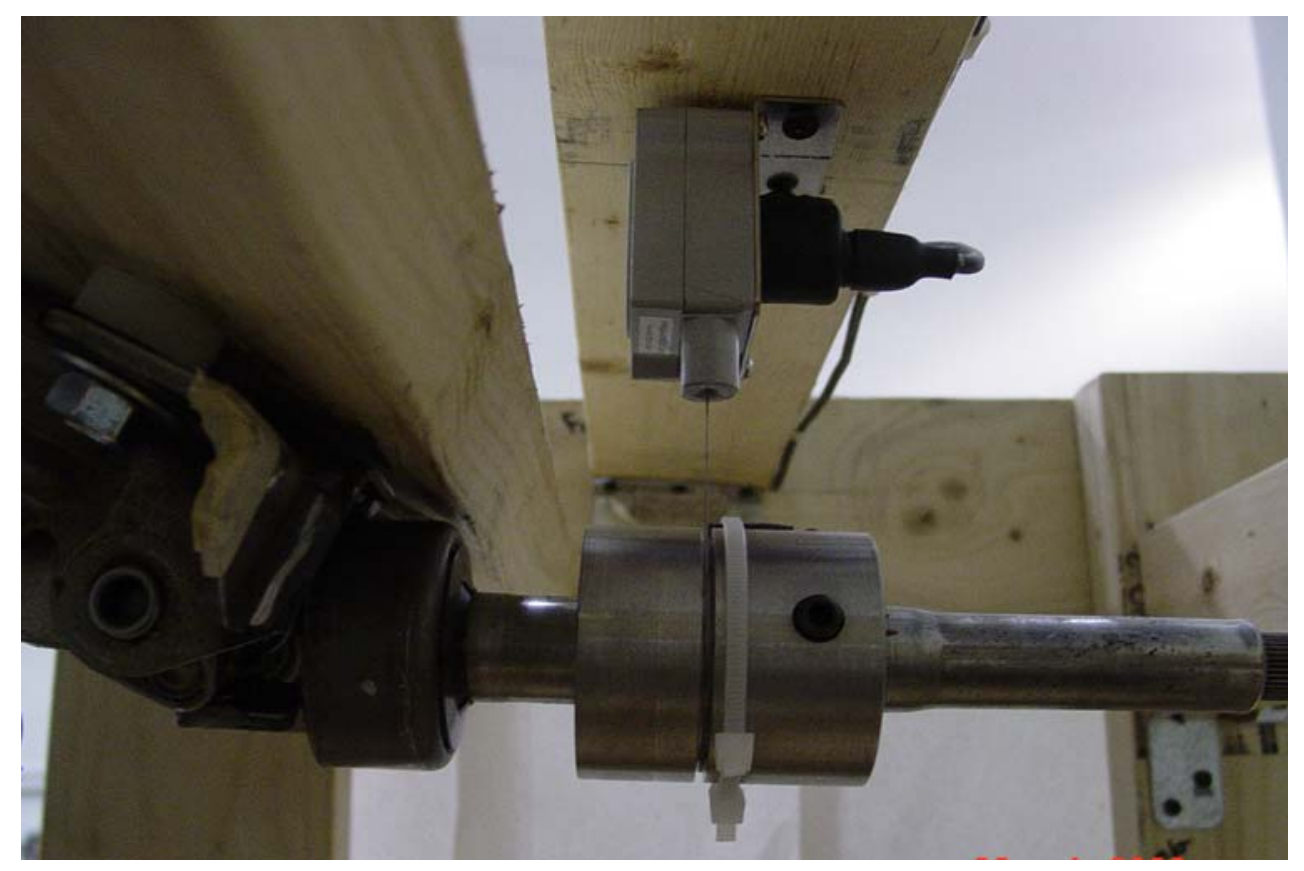

Figure 6.6. Celesco SP2-25 string pot and steel collar

The steering wheel angle measurement was accomplished via a string pot. A string pot is a device that utilizes a flexible cable, a spring-loaded spool and a potentiometer to detect and measure linear position [34]. In the case of an angular measurement the string is wrapped around a shaft, in this case the steering shaft, and according to the Celesco company website, this string pot is recommended for applications involving steering wheel angle measurement. The steel collar, shown in Figure 6.6, above, slides over the steering shaft and contains a machined groove to contain the string as it wraps around the shaft. This groove provides a constant diameter and prevents the string from wandering, thus keeping it in the same location along the shaft. The end of the string is mounted in a machined groove on the collar. This particular sensor, a Celesco SP2-25 is 25 inches long.

Summary of major support equipment being used:

1. dSPACE MicroAutoBox 1401/1501

2. RadioShack 3 amp $13.8 \mathrm{~V}$ power supply

3. $10 \mathrm{amp}$ inline fuse on positive power supply wire between power supply and dSPACE

4. Laptop running CarSim RT, Matlab / Simulink, and dSPACE ControlDesk

5. Steering wheel and shaft from full size passenger vehicle

6. Celesco SP2-25 string pot and steel collar

In Chapter 5 TruckSim 7 was used as the simulation program to validate the 3 degrees of freedom model developed in Matlab. As was stated earlier, this simulator aims to fully integrate the driver's input and allow him to fully participate in the algorithm development, all the while eliminating many of the common in-vehicle testing variability using a controlled laboratory environment. This requires the ability to run in real time. In order to run in real-time a real-time version of TruckSim needed to be used, i.e. TruckSim RT. However, it was found that 
Mechanical Simulation Corp. does not support the dSPACE MicroAutoBox with TruckSim RT due to computational speed requirements [32]. They do, however, support BikeSim RT and CarSim RT. Since the current steering model that is being built in Simulink would be nearly the same regardless of whether CarSim RT or TruckSim RT was being used, it was decided CarSim RT would be the simulation program used for the steering HIL development. If in the future a dSPACE system that is compatible with TruckSim RT is used, the only difference to the Simulink steering model will be a simple S-function used in the Simulink model.

The Simulink model shown in Figure 6.7 was built based on the "ABS_Example" in CarSim. The rationale was that if this model worked and only the steering wheel angle needed to be added as an import and export variable, the resulting Simulink model should be capable of steering the real-time animated vehicle.

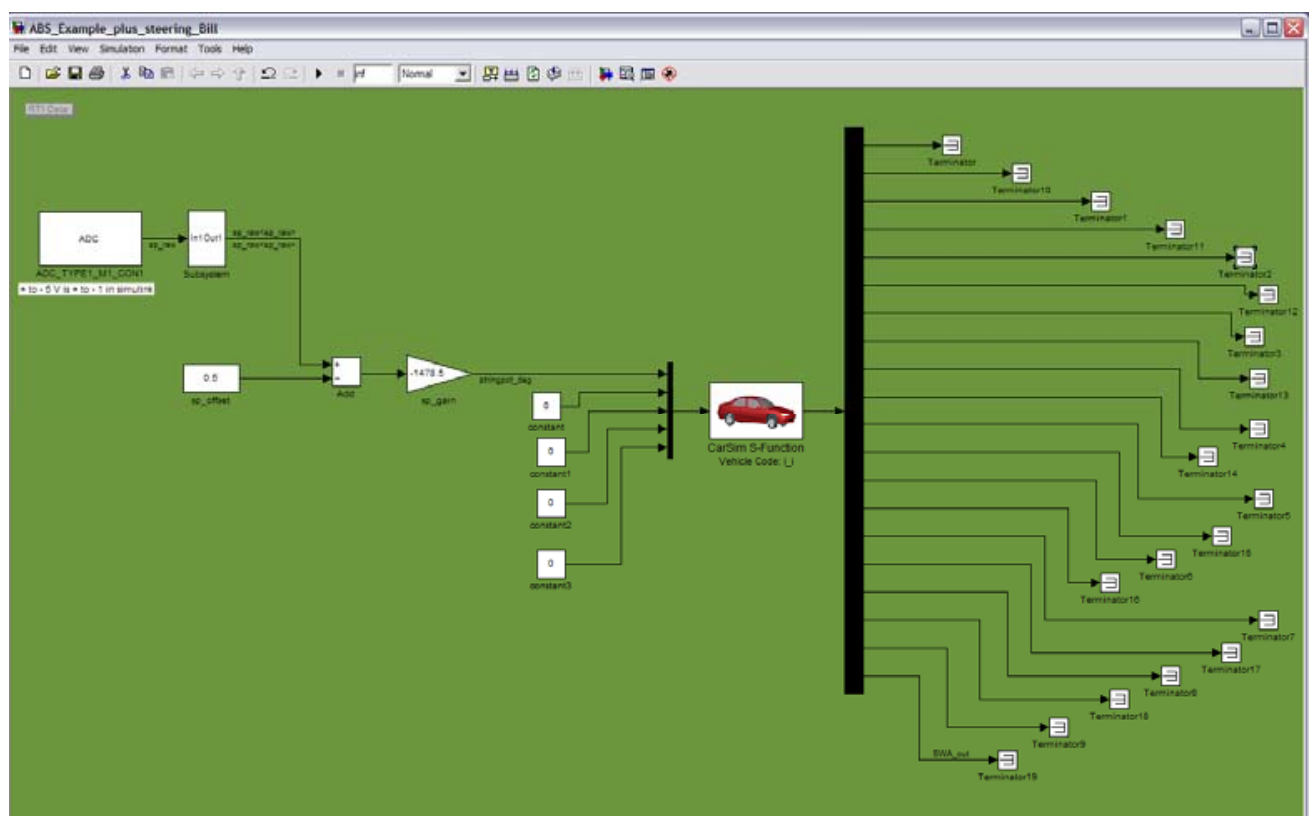

Figure 6.7 : Simulink model used for the steering functionality 


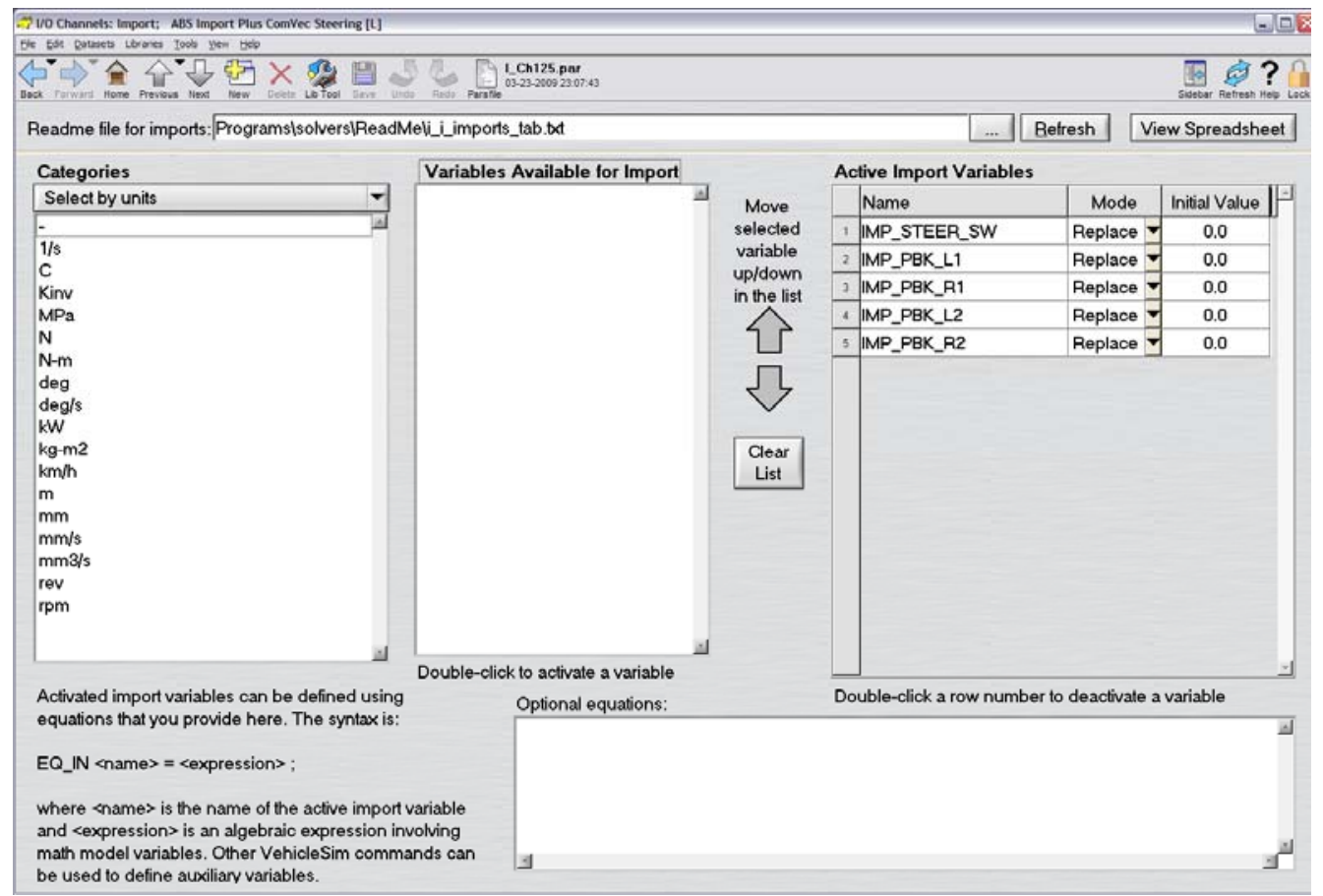

Figure 6.8 : Import Channel Screen in CarSim RT. Note variable 1, IMP_STEER_SW in the Active Import Variables list. This is the steering wheel angle, measured in degrees.

Since it was not desirable at this time to include the default brake signals from the "ABS_Example” model, all of the existing ABS import signals were set to zero and all of the export variables, including the steering, were attached to Terminator blocks in the Simulink model.

CarSim RT has two separate windows for adding import and export variables. The Import Channel screen, shown in Figure 6.8, is where the user can include all variables that will be inputs to the CarSim S-function in Simulink. The CarSim variable IMP_STEER_SW located on the Import Channels screen was added, and the mode was chosen as "replace". The "replace" mode is the standard choice for any signal that is being read into Simulink via dSPACE. This means that CarSim will be looking for new values of steering wheel angle at each time step to replace the previous value. It is also important to note the location of IMP_STEER_SW in the "Active Import Variables" list. This location must be the same as in the mux block in Simulink just prior to the CarSim S-function. See Figure 6.7. 


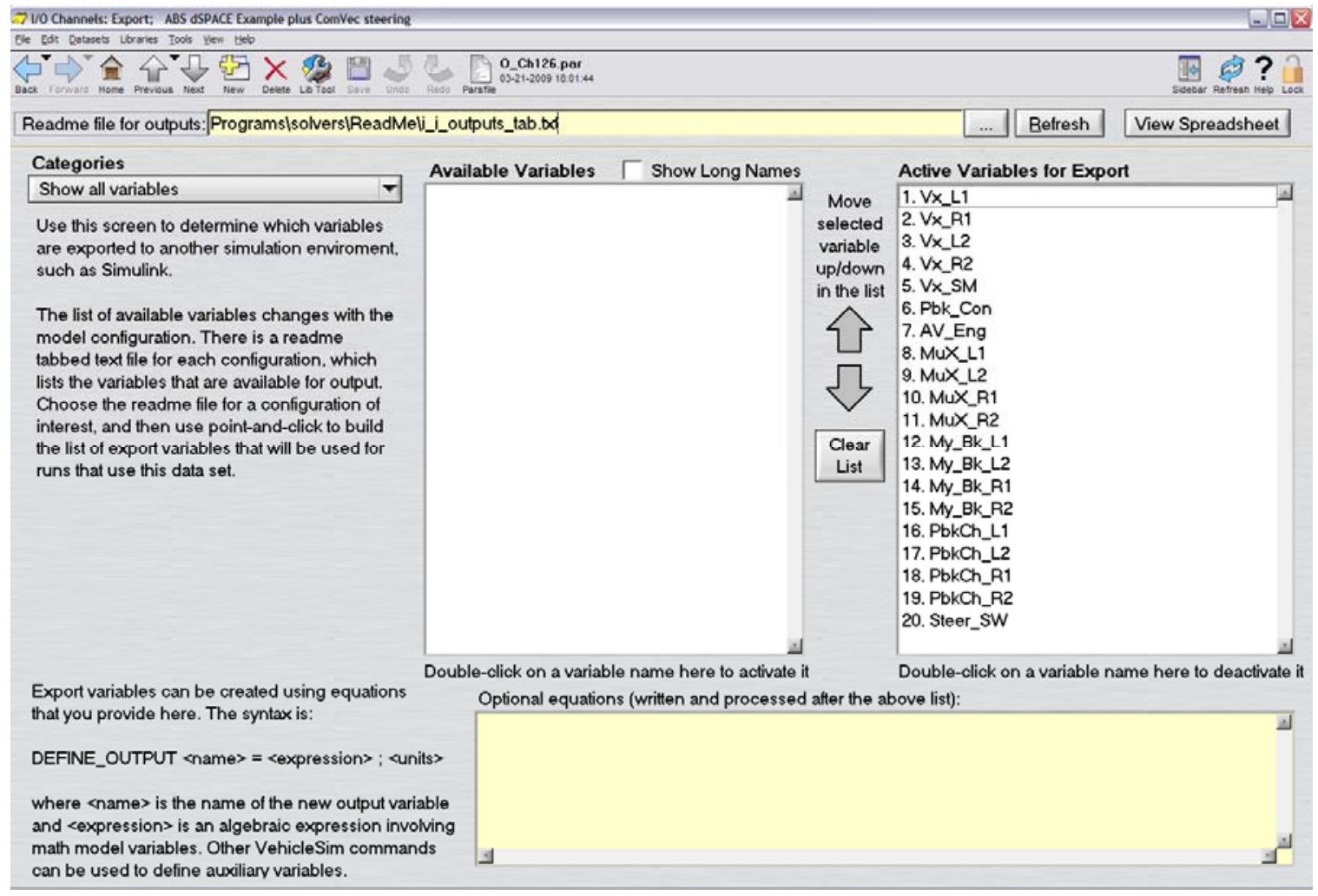

Figure 6.9 : Export Channel screen in CarSim RT. Note variable number 20, STEER_SW in the Active Variables for Export list. This is the steering wheel angle, measured in degrees.

The Export Channel screen from CarSim RT is shown in Figure 6.9. Using the Export Channels page, the steering wheel angle variable Steer_SW was chosen. This means that the CarSim Sfunction is going to export the steering wheel angle to be used further along in the Simulink model. For the current model this is not necessary since steering wheel angle is not being used to control anything else nor is it being used as a feedback signal. Therefore, in the Simulink model, this output signal is attached to a Terminator block. Again, note the location of Steer_SW in the “Active Variables for Export” list and its corresponding location in the demux block in Simulink in Figure 6.7. 


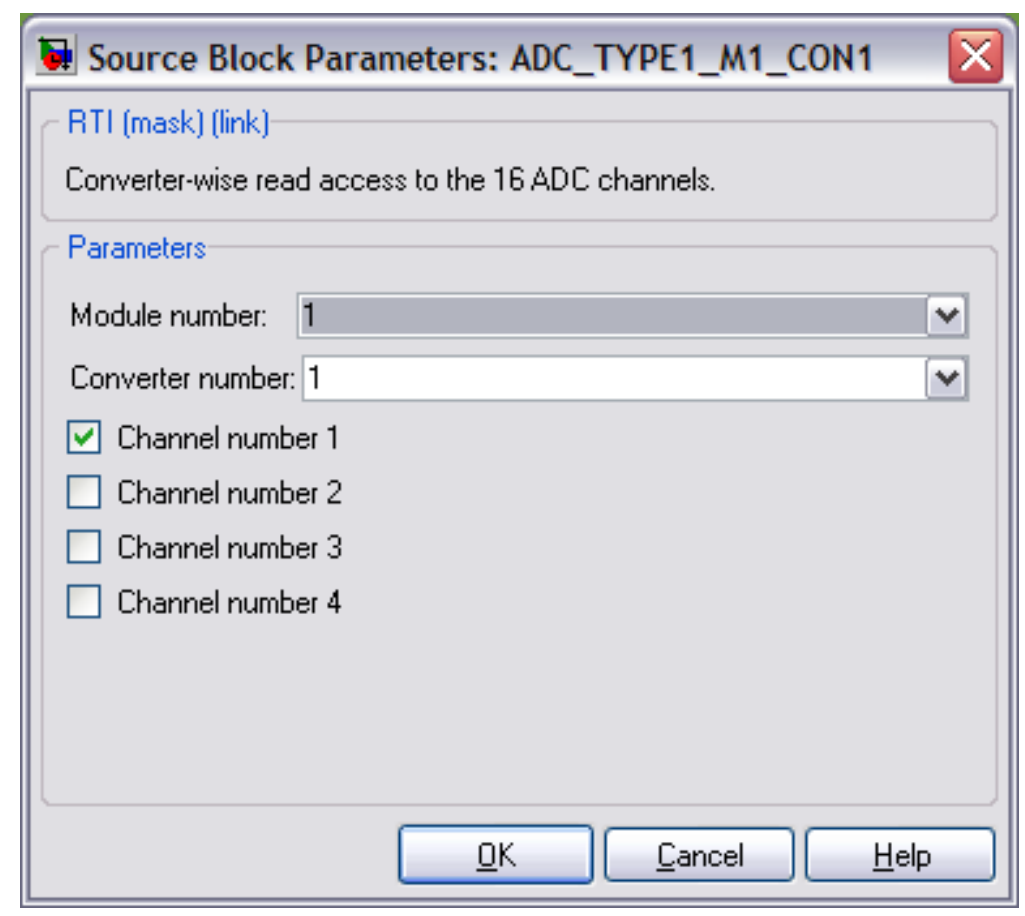

Figure 6.10 : Source Block Parameters for Analog / Digital Converter block in Simulink. The block is an ADC_TYPE1_M1_CON1

The other necessary block in the Simulink model is the ADC block, or Analog / Digital Converter block. This block accepts the dSPACE signals which in this case is the string pot data. The block is configured to read Module number 1, Converter number 1 , Channel number 1 which matches the signal location on the dSPACE connector, location c1.

Once all of this had been established it was necessary to determine both a string pot offset and string pot gain. The string pot reads the steering input that will result in turning the simulated vehicle both left and right, corresponding to positive and negative steering angle. The offset is necessary because the string extension corresponding to SWA $=0$, or straight-ahead driving, would be the midpoint of the string. This means that the string would already be extended to half its length for SWA $=0$. Therefore the offset would have to be -0.5 , or $-50 \%$ of its length. The negative sign is there to subtract 0.5 from the string pot signal. Thus subtracting 0.5 had the effect of zeroing the steering wheel angle signal. 


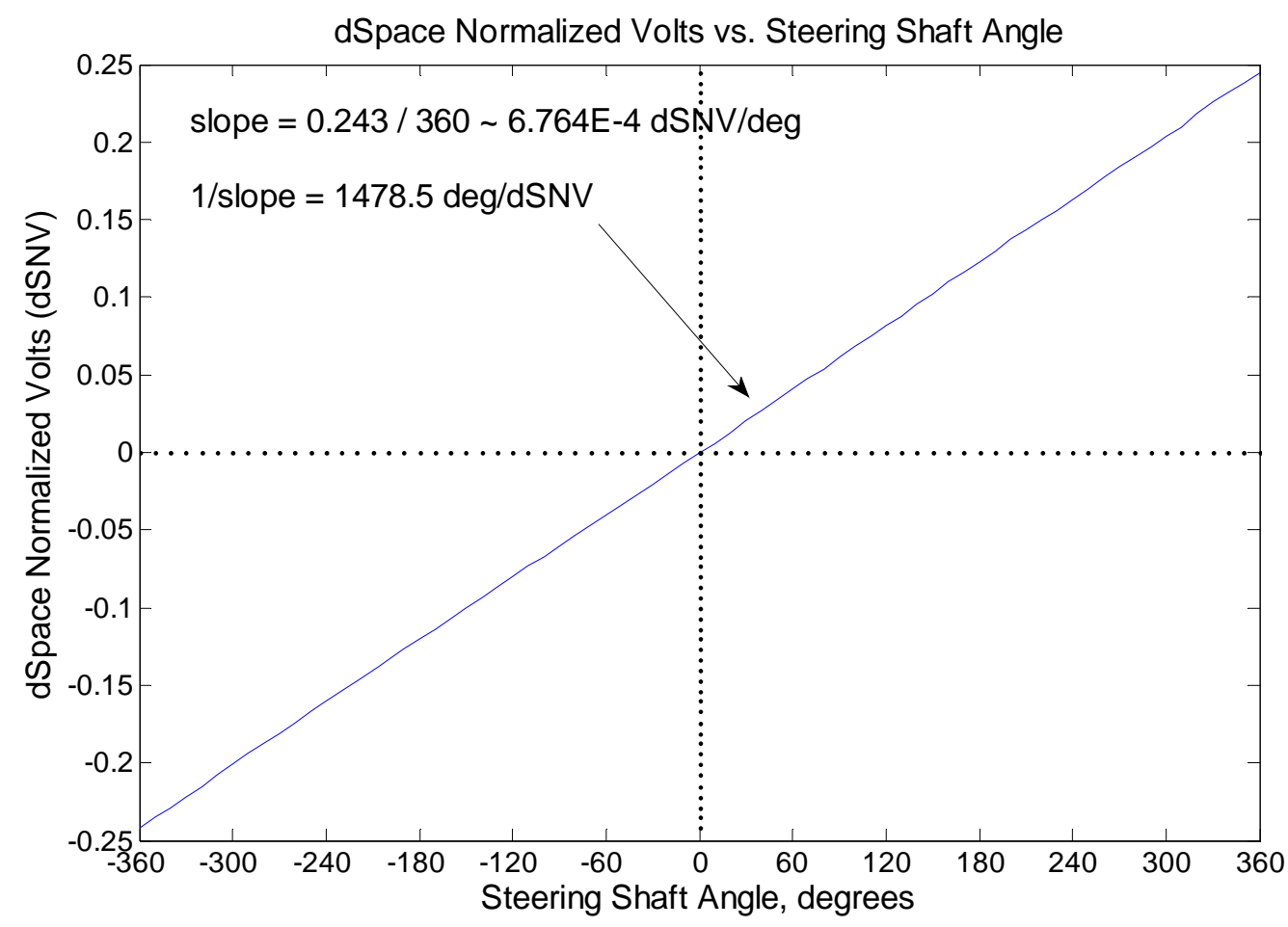

Figure 6.11 : Stringpot signal output as read by dSPACE vs. Steering shaft rotation

The stringpot gain of 1478.5 was determined by measuring the string pot signal output as read by dSPACE in what are referred to here as "dSPACE Normalized Volts" for every 10 degrees of steering shaft rotation in both the negative and positive steering directions. It should be noted that due to coordinate system differences between the 3DOF Matlab simulation presented in Chapter 5 and CarSim, left steering is positive in CarSim and negative in the 3DOF Matlab simulation. It can be seen that this trend is very linear; in fact for every 10 degrees of steering shaft angle the dSPACE Normalized Volts (dSNV) increase by $0.007+/-0.001 \mathrm{dSNV}$.

The gain was changed to -1478.5 in the Simulink model of Figure 6.7 to correct for the fact that initially, turning the steering wheel to the left resulted in the CarSim vehicle turning to the right. This could be physically corrected by moving the string pot to the other side of the steering shaft.

Order of operations:

Step 1. Begin with CarSim. Several example Simulink models are available via the CarSim main screen. In this case an existing Simulink model was chosen and modified to include the necessary steering variables to the Import and Export channels. Also important to make sure the files are in the correct paths in CarSim.

Step 2. Open the newly created Simulink model from the CarSim main screen. This opens the Simulink model with the CarSim S-function. Using the Simulink Library add any necessary model components for the system being tested. 


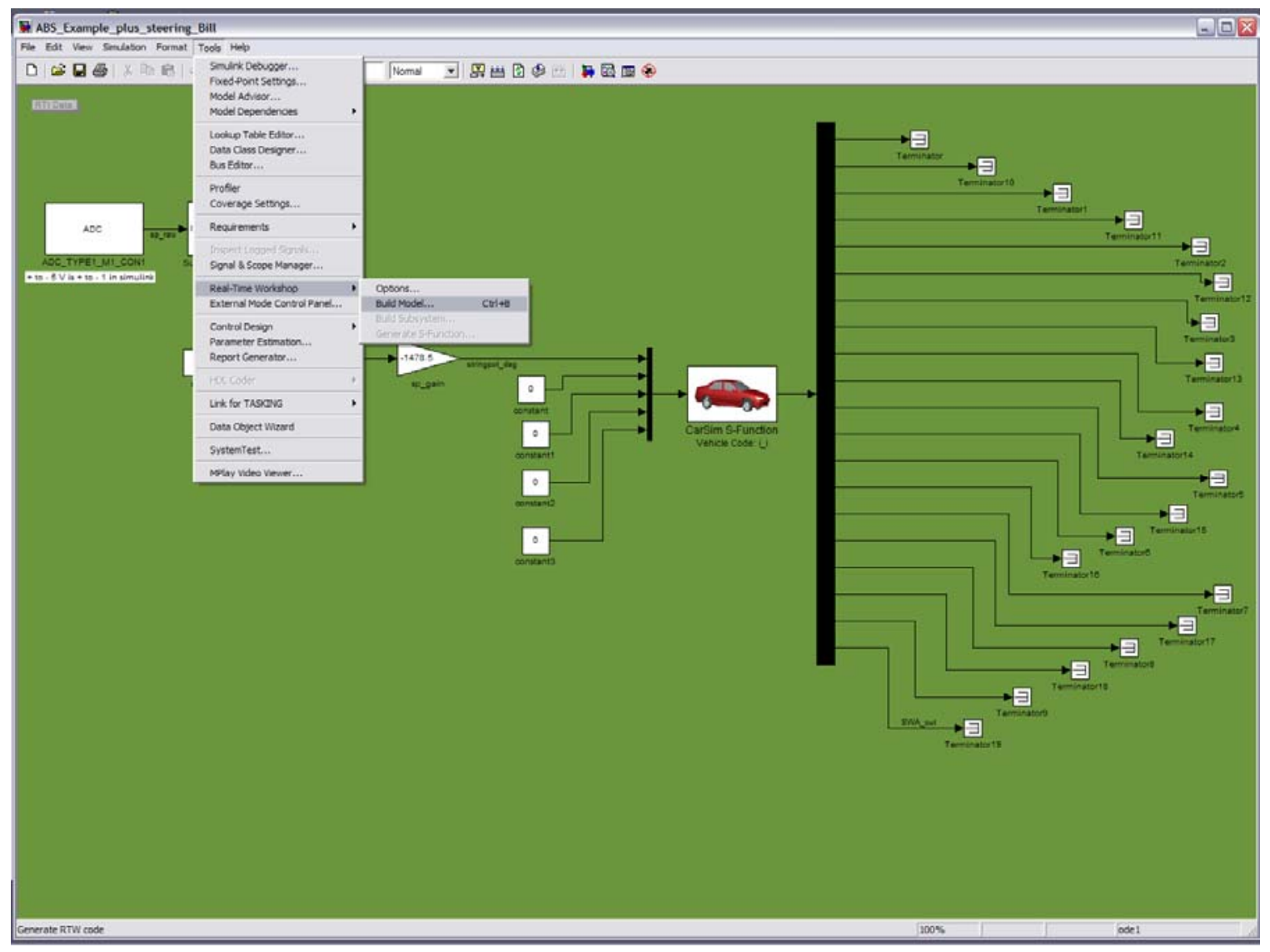

Figure 6.12: Build Model option in the Tools, Real Time Workshop menu

Step 3. In the Tools drop down menu, select the Real Time Workshop, Build Model option in Simulink, shown in Figure 6.12. This process compiles the Simulink and CarSim information. CarSim features such as vehicle type, speed, or animation run time can be changed in CarSim.

Step 4. Upon completion of Step 3 return to the CarSim main screen and select either Run or Load/Run. The Load/Run option opens the dSPACE ControlDesk first, then the animation. Selecting the Run option just opens the animation. It is important to note that the Build Model function in Simulink only has to be run if something is changed in the CarSim Import or Export channels, or if something is changed in the Simulink model. If changes are only made in CarSim, such as vehicle speed or type, the Build Model option does not need to be repeated. 


\subsection{HIL Simulator Results}

Once all of the equipment was connected the CarSim RT vehicle could be steered. Figure 6.13 shows the CarSim vehicle performing straight ahead driving with the steering wheel in the straight-ahead (i.e. SWA = 0) position. The chosen surface is a $1 \mathrm{~km}$ square. For reference, note the fore-aft grid line running along the horizontal centerline of the vehicle.

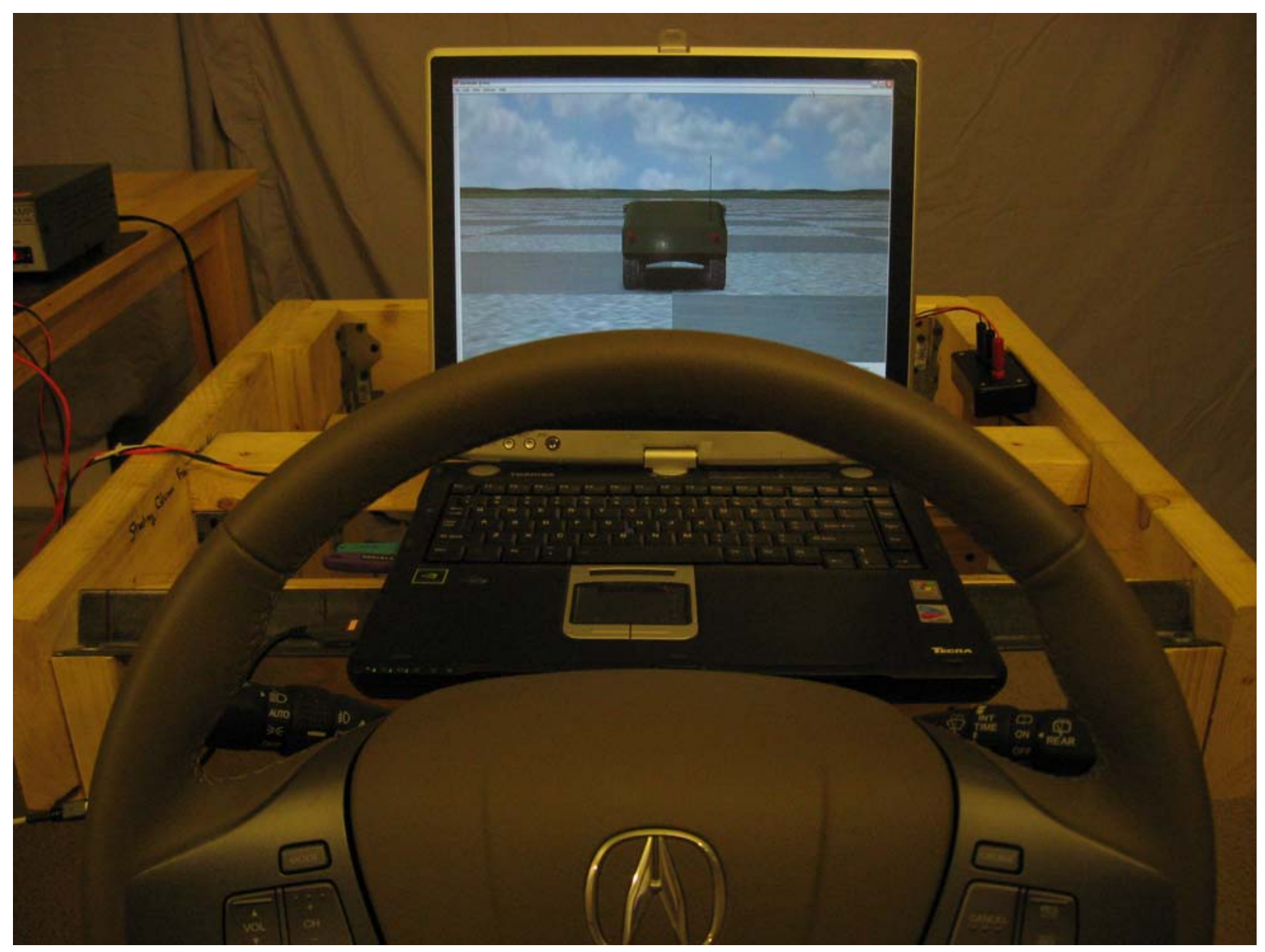

Figure 6.13 : CarSim RT vehicle in straight ahead driving. Vehicle simulated is an HMMWV (military HUMVEE). 


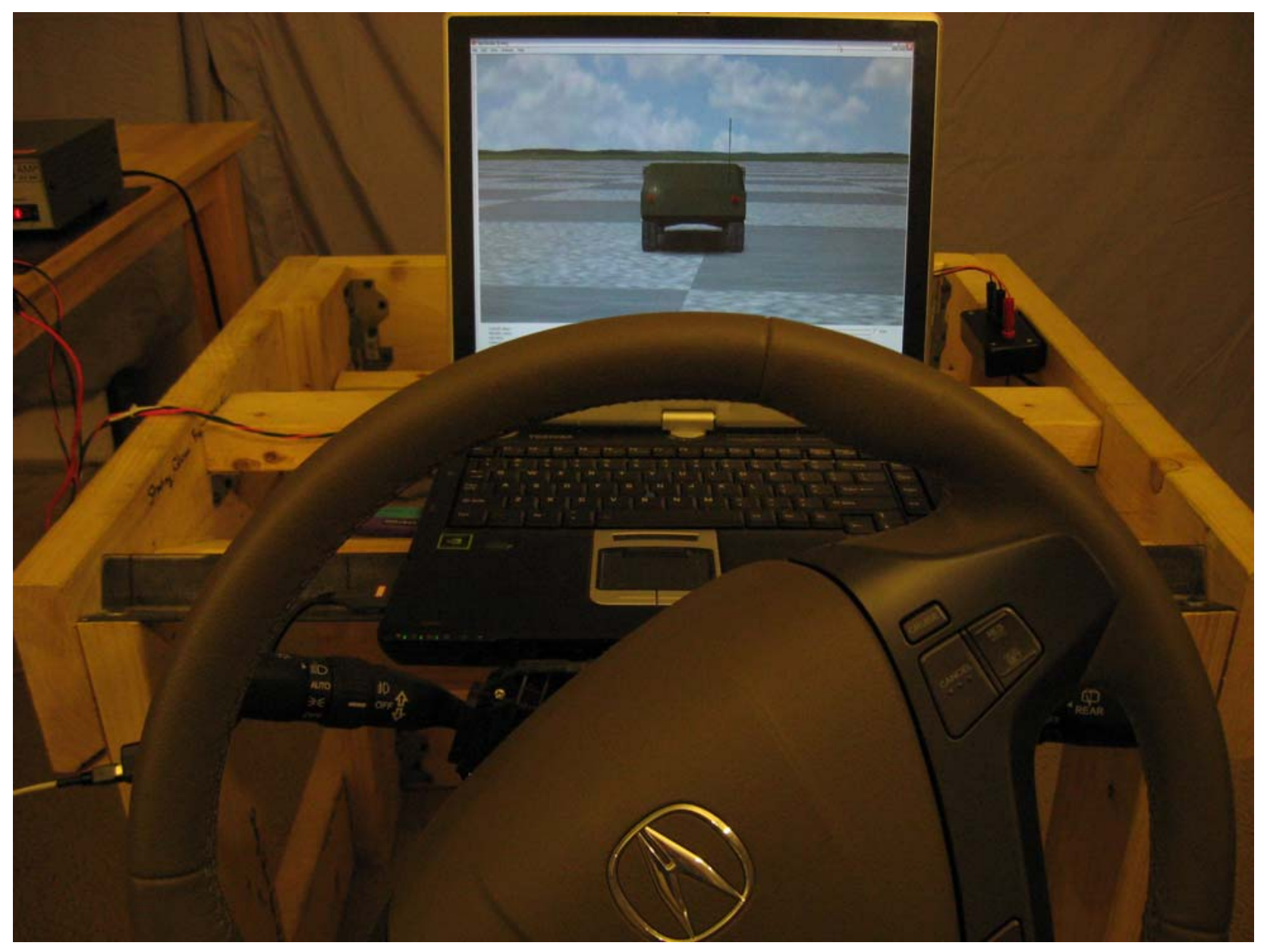

Figure 6.14 : CarSim RT vehicle in a left turn

Figure 6.14 shows the CarSim RT vehicle executing a left turn with the steering wheel in the left turning position. For reference, note the orientation of the vehicle with respect to the grid line on the road surface. In this case, the vehicle has moved to the left of this line. 


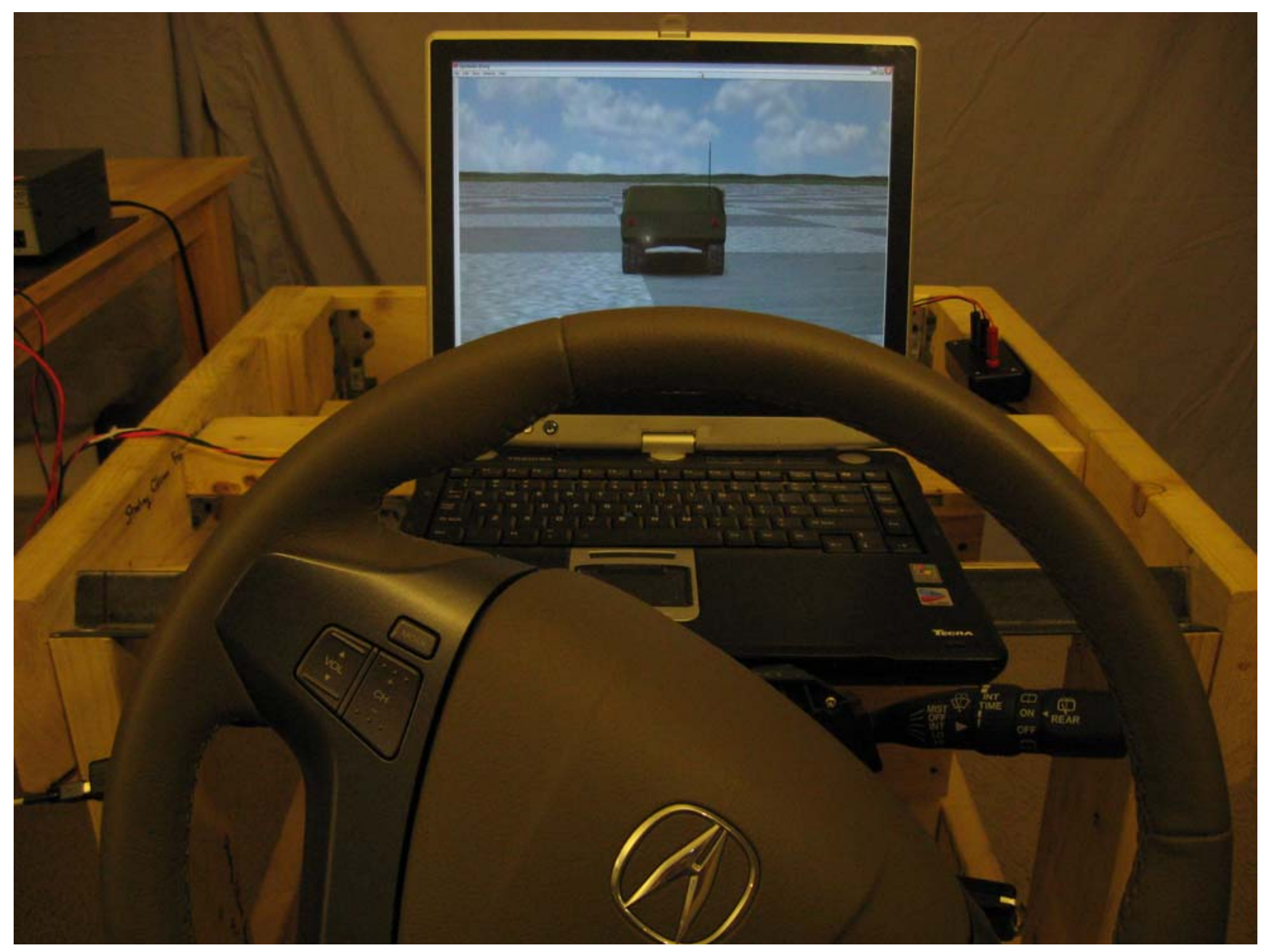

Figure 6.15 : CarSim RT vehicle in a right turn.

Figure 6.15 shows the CarSim RT vehicle executing a right turn with the steering wheel in the right turning position. For reference, note the orientation of the vehicle with respect to the grid line on the road surface. In this case, the vehicle has moved to the right of this line. 


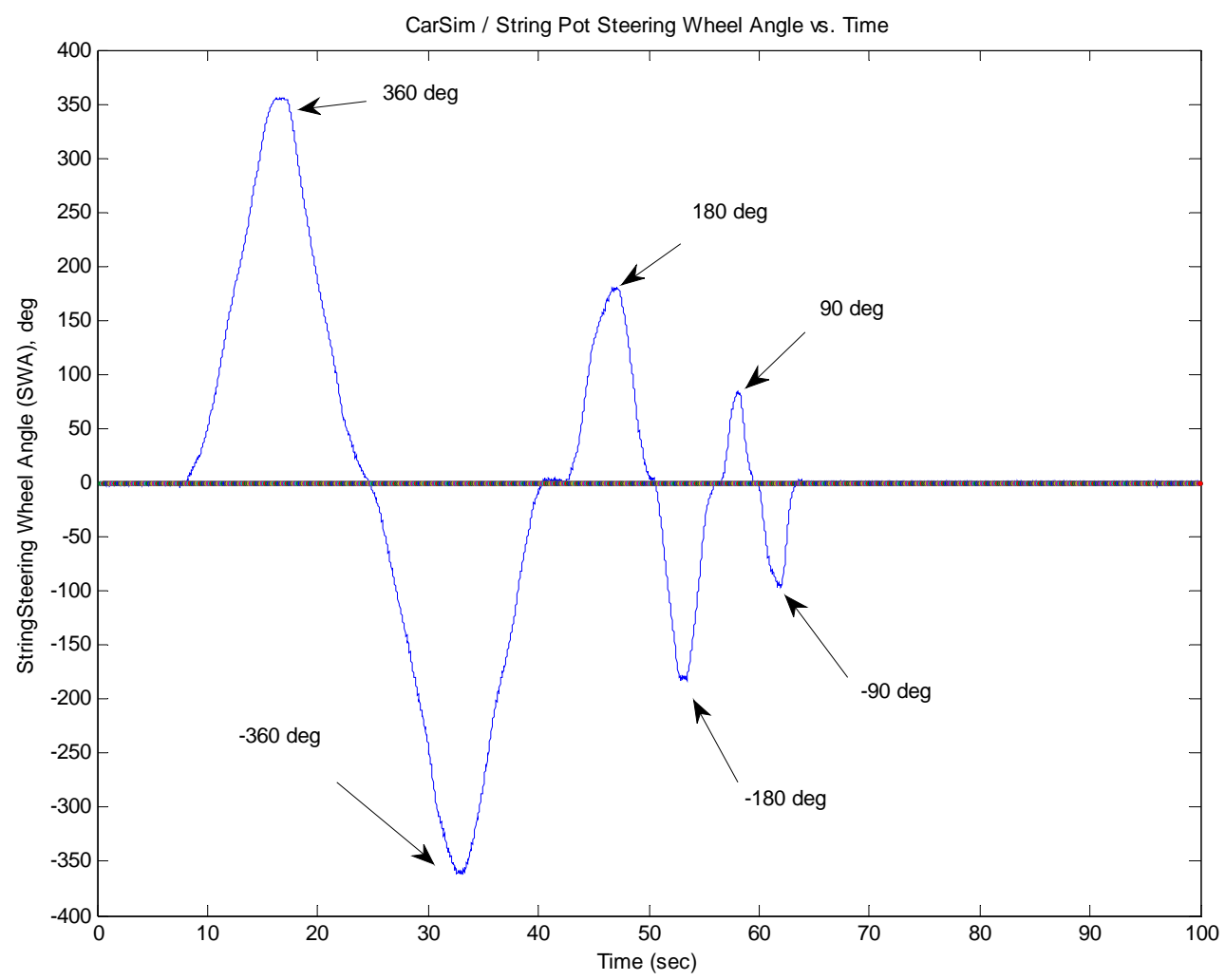

Figure 6.16 : CarSim / String Pot Steering Wheel Angle vs. Time

Figure 6.16 shows the CarSim / String Pot Steering Wheel Angle vs. Time data. This is the CarSim output channel Steer_SW. As can be seen, for different steering inputs the steering HIL system provides acceptable results. This proves that the gain of $(-1478.5 \mathrm{dSNV} / \mathrm{deg})$ is valid for all steering angles between 0 and $+/-360 \mathrm{deg}$. Note that since the string pot output is linear, steering angles beyond +/- 360 degrees could be achieved with the same gain with a good degree of certainty. However, it would be wise to confirm this if steering angles in these ranges were desired.

\subsection{HIL Simulator Future Development}

As mentioned in the Introduction and Technical Overview at the beginning of this chapter, the ability to steer the CarSim RT vehicle was step 1 in a 3 step process. Suggested steering enhancements have included an active steering control similar to what is currently available on some BMW models as well as steering torque and tire force feedback akin to what was covered in [29]. One option might be to incorporate additional CarSim signals such as tire aligning moments and tire slip angles and use those signals to generate a feedback steering shaft torque. Another idea would be to use an electromagnet to generate the necessary reaction torque on the shaft, with a controller being built based on the aforementioned CarSim tire aligning moments and slip angles. Once implemented the steering torque vs. steering wheel angle could be evaluated, tuned, and even compared to real vehicle data using a Lissajous Curve [29]. 
Step 2 would be to incorporate throttle control. Since the accelerator pedal, visible in Figures 6.1-6.4, is already in place a method to read the pedal position is needed. Several options include a string pot attached to the front of the pedal, or a linear potentiometer placed behind the pedal and measuring the linear pedal travel directly. Since the pedal does travel through an arc the total angular displacement would have to be considered. If this angle was too large for a linear sensor to be used, another option would be opening the pedal case and attaching a rotary potentiometer to the pedal hinge pin. In any of these cases, this signal would be read into the Simulink model via dSPACE in the same manner as the steering string pot.

Step 3 would be the fully functioning braking system. As currently envisioned, this task would be broken down into multiple projects, beginning with the brake pedal position and proceeding first with a non-hydraulic braking system using just the pedal position as an input to the Simulink model using CarSim brake pressures. Once a functioning brake system ECU/HCU unit was available, a non-ABS hydraulic system could be developed followed by the inclusion of ABS, TCS, and finally ESC functionality.

Once the simulator is fully functional from a vehicle dynamics standpoint, the next step would be to include a GPS signal so that the TSM developed in this thesis could be evaluated, tuned, and validated. In [30] Kim, Lee, and Yu discussed the development of a vehicle simulator using a GSS6560 real-time GPS simulator from Spirent Corp. Their goal was telematics software development but, based on the information available from Spirent Corp.'s website, this GPS simulator would be capable of satisfying our needs. 


\section{Chapter 7}

\section{Conclusions}

After an overview of GPS technology with a focus on its implementation in vehicle dynamics studies, the linear, single track three degree-of-freedom (3DOF) dynamic equation set was derived in detail. Starting from first principles, this derivation successfully filled in the gaps left by Pacejka [12]. Specifically, these were the steps necessary to obtain the four Lagrange equations, the equations for the generalized forces and virtual work, and a determination of the Euler angle-based rotation matrices. The three angular velocity equations were obtained, from which the full equations for the kinetic and potential energy were found. The derivation concluded with the three degree-of-freedom (3DOF) equation set in state space form.

The results of the Matlab and TruckSim vehicle simulation studies, as well as the fuzzy logic application using a right sigmoid membership function, were presented in Chapter 5. A new fuzzy logic-based predictive control methodology was developed which is capable of predicting the vehicle instability level through the use of GPS data and predictive vehicle modeling. The simulation results for five vehicle masses at three longitudinal velocities in both Matlab and TruckSim, as well as the five additional data points for the wheel lift study, showed the applicability of the methodology for heavy trucks, where the output of the fuzzy controller was used as inputs to the Total Safety Margin, or TSM, in the form of the safety margins lm, vm, rm, and pm (Lateral Acceleration, Lateral Velocity, Yaw Rate, and Roll Angle, respectively). Weighting factors were determined by comparing the maximum values of the TruckSim $80 \mathrm{kph}$ and Wheel Lift Study results. These were used in the TSM to scale each term to reflect rule credibility, or in this case the importance of each dynamic response specific to the vehicle type being modeled. Using these weighting factors, the TSM Matrices for both the 3DOF and TruckSim simulations were presented in Tables 5.13 and 5.14. These matrices showed similar results with low percent errors, indicating that the 3DOF model is capable of predicting the vehicle instability level close to TruckSim.

Chapter 6 presented the development of the hardware-in-the-loop simulator currently under development at the Virginia Tech Center for Vehicle Systems and Safety (CVeSS). Using CarSim RT, Simulink, dSPACE ControlDesk, a steering column from a production vehicle, and a string pot, the functionality of the steering module was successfully demonstrated by steering the CarSim RT vehicle accurately to +/- 360 degrees of steering wheel angle (SWA). 


\section{Chapter 8}

\section{References}

[1] Naranjo, Jose E et al, "Fuzzy Logic Based Lateral Control for GPS Map Tracking”, Madrid, Spain: Spanish Ministry of Fomento, Spanish Ministry of Science and Technology, Citroen Spain S.A., 2003.

[2] Kaplan, Elliot D., Hegarty, Christopher J., "Understanding GPS Principles and Applications, $2^{\text {nd }}$ ed.”, Artech House Publishers, 2006.

[3] Venhovens, P.J. Th., Bernasch, J.H., Lowenau, J.P., Rieker, H.G., Schraut, M., "The Application of Advanced Vehicle Navigation with BMW Driver Assistance Systems”, SAE technical paper 1999-01-0490, 1999.

[4] Logsdon, Tom, “Understanding the NAVSTAR GPS, GIS, and IVHS, $2^{\text {nd }}$ ed.”, Van Nostrand Reinhold, 1995.

[5] Hofmann-Wellenhof, B., Lichtenegger, H., Collins, J., "GPS Theory and Practice, $5^{\text {th }}$ ed.", Springer-Verlag Wien New York, 2001.

[6] Beiker, Sven A., Gaubatz, Karl Heinz, Gerdes, J. Christian, Rock, Kirsten L., "GPS Augmented Vehicle Dynamics Control”, SAE technical paper 2006-01-1275, 2006.

[7] Dixon, John C., “Tires, Suspension and Handling $2^{\text {nd }}$ ed.”, Warrendale, PA, Society of Automotive Engineers, 1996.

[8] Gillespie, Thomas D, "Fundamentals of Vehicle Dynamics", Warrendale, PA, Society of Automotive Engineers, 1992.

[9] Jazar, Reza N., "Vehicle Dynamics: Theory and Application”, New York, Spring Science+Business Media, LLC, 2008.

[10] Karnopp, Dean, “Vehicle Stability”, New York, Marcel Dekker, Inc., 2004.

[11] Milliken, William F., Milliken, Douglas L., "Race Car Vehicle Dynamics”, Warrendale, PA, Society of Automotive Engineers, 1995. 
[12] Pacejka, Hans B, "Tire and Vehicle Dynamics $2^{\text {nd }}$ ed.", Warrendale, PA, Society of Automotive Engineers, 2006.

[13] Wong, J.Y, “Theory of Ground Vehicles $3^{\text {rd }}$ ed.”, New York, John Wiley and Sons, Inc., 2001.

[14] Sidi, Marcel J., "Spacecraft Dynamics and Control: A Practical Engineering Approach”, New York, Cambridge University Press, 2002.

[15] Riede, Peter M., Leffert, Ronald L., Cobb, William A., “Typical Vehicle Parameters for Dynamic Studies Revised for the 1980’s”, SAE technical paper 840561, 1984.

[16] Isik, Can, "Fuzzy Logic: Principles, Applications, and Perspectives”, SAE technical paper 911148, 1991.

[17] Hessburg, Thomas, and Masayoshi Tomizuka, “A Fuzzy Rule-based Controller for Automotive Vehicle Guidance”, Berkeley, University of California, 1991.

[18] Boada, B.L., Boada, M. J. L. and Diaz, V. (2005) "Fuzzy-logic applied to yaw moment control for vehicle stability”, Vehicle System Dynamics.

[19] Abbasi, Ebrahim, Abouec, Amir (2008) "Stock Price Forecast by Using Neuro-Fuzzy Inference System”, Proceedings of World Academy of Science, Engineering and Technology, Volume 36, December 2008.

[20] Kosko, Bart, “Fuzzy Engineering”, Upper Saddle River, Prentice Hall, 1997.

[21] Klir, George J. and Bo Yuan, "Fuzzy Sets and Fuzzy Logic: Theory and Applications", Upper Saddle River, Prentice Hall, 1995.

[22] Piegat, Andrezej, “Fuzzy Modeling and Control”, Physica-Verlag Heidelberg, 2001.

[23] Ehlbeck, Jim, Kirn, Chris, Moellenhoff, Joerg, Korn, Alan, Rosendahl, Harmut, Ruhnau, Gerhard, "Freightliner/MeritorWABCO Roll Advisory and Control System”, SAE technical paper 2000-01-3507, 2000.

[24] Duprey, Benjamin, Tamaddoni, Seyed Hossein, Taheri, Saied, "A New Fuzzy Based Stability Index Using Predictive Vehicle Modeling and GPS Data”, SAE technical paper 200801-2597, 2008.

[25] Sorniotti, Aldo, Velardocchia, Mauro, "Hardware-in-the-Loop (HIL) testing of ESP (Electronic Stability Program) Commercial Hydraulic Units and Implementation of New Control Strategies", SAE technical paper 2004-01-2770, 2004.

[26] Velardocchia, Mauro, Sorniotti, Aldo, "Hardware-in-the-Loop to Evaluate Active Braking Systems Performance”, SAE technical paper 2005-01-1580, 2005. 
[27] Kohl, Susanne, Jegminat, Dirk, "How to do Hardware-in-the-loop Simulation Right”, SAE technical paper 2005-01-1657, 2005.

[28] Schuette, Herbert, Waeltermann, Peter, "Hardware-in-the-Loop Testing of Vehicle Dynamics Controllers - A Technical Survey”, SAE technical paper 2005-01-1660, 2005.

[29] Segawa, Masaya, Nakano, Shirou, Shino, Motoki, Nagai, Masao, "Preliminary Study Concerning Quantitative Analysis of Steering System using Hardware-in-the-Loop (HIL) Simulator”, SAE technical paper 2006-01-1186, 2006.

[30] Kim, Moon-Sik, Lee, Soo-Young, Yu, Si-bok, “Development of a Vehicle Simulator Based Testing Method for Telematics Software Development”, SAE technical paper 2007-01-0945, 2007.

[31] Mechanical Simulation Corporation, “TruckSim 7”, www.carsim.com, 2008.

[32] Mechanical Simulation Corporation, “CarSim RT”, www.carsim.com, 2008.

[33] Personal communication with Dr. Tom Gillespie, 2009.

[34] Celesco Transducer Products, Inc., www.celesco.com, 2009.

[35] Khanafer, Ali, Balzer, Dirk, Isermann, Rolf, “A Rule-based Collision Avoidance System Scene Interpretation, Strategy Selection, Path Planning and System Intervention” SAE technical paper 2009-01-0156.

[36] Davoudi, Mohsen, Menhaj, Mohannad Bagher, Davoudi, Mehdi. “A Fuzzy Based Vehicle Dynamic Stability Control (FDSC)”, SAE technical paper 2006-01-3483.

[37] Bernard, James, Shannan, Jay, Vanderploeg, Martin, "Vehicle Rollover on Smooth Surfaces”, SAE technical paper 891991.

[38] Gottwald, Carl, “Decision Making in Preliminary Design”, SAE technical paper 730889.

[39] Rollover FAQs, http://www.safercar.gov. 


\section{Appendix A}

\section{Definitions of Terms}

2 degree-of-freedom (DOF) linear, single track model : A mathematical representation of a vehicle in which small steering and tire slip angles are assumed. In this instance the radius of curvature is assumed to be large and thus the left and right side tires are combined into a single front and single rear tire, like a bicycle. A linear tire is assumed, meaning that the tire slip angle remains small, typically below about three degrees. The two degrees of freedom for this model are lateral velocity and yaw rate [7-13]. See Chapter 4.

3 degree-of-freedom linear, single track model : A mathematical representation of a vehicle with the same steering and tire assumptions as the 2 DOF model, but with an additional degree of freedom. In this thesis, the roll angle of the sprung mass was considered. See Chapter 4.

a : horizontal distance between front axle and vehicle center of gravity (m)

ABS : Antilock Braking System. An electronic braking system (EBS) which functions to modulate the wheel speeds by varying brake pressures to keep the wheels near their peak friction levels on all surfaces, especially under rapid brake pedal applications. Especially useful on reduced road friction surfaces such as gravel, rain, snow, and ice. The goal is to prevent the wheels from locking up, i.e. wheel speeds reducing to zero while the vehicle is still moving. Complexity and sophistication vary by vehicle manufacturer. Major suppliers include Continental Corporation, Bosch, and TRW Automotive.

b : horizontal distance between rear axle and vehicle center of gravity (m)

Bicycle Model: single track model [7-13]. See Chapter 4.

CG : vehicle center of gravity

$\mathrm{C}_{\alpha}$ : tire cornering stiffness $(\mathrm{N} / \mathrm{rad})$

$C_{\phi}:$ total roll damping (Nms/rad)

DOF : degrees of freedom

ECU : electronic control unit. A generic term used for any computer used to run a vehicle subsystem such as electronically controlled brakes, engine, transmission, or drivetrain components such as an electronically controlled differential.

EBS : electronic braking system 
EBS Sensors : Includes the wheel speed sensors for ABS and yaw rate gyroscope and steering wheel angle for the electronic stability control. Roll Stability Control will add a roll rate or similar sensor. Other sensors may be included depending on vehicle manufacturer.

ESC : electronic stability control, originally ESP (Electronic Stability Program). Vehicle Manufacturer-specific names are vast and include Vehicle Stability Assist (VSA, Honda), AdvanceTrac (Ford), and StabiliTrak (GM). Electronic Stability Control is primarily a yaw control system designed to prevent the vehicle from understeering or oversteering beyond certain yaw thresholds. This is accomplished by building upon existing EBS hardware and software. An additional input beyond ABS is steering wheel angle. Major suppliers include Continental Corporation, Bosch, and TRW Automotive.

$F_{y F, R}:$ tire lateral force $(\mathrm{N})$

$F_{x F, R}$ : tire tractive force $(\mathrm{N})$. Tractive and braking forces, i.e. longitudinal tire forces are not included in this study.

Fuzzifier : This block of code uses a right sigmoid membership function, one for each of Lateral Acceleration, Lateral Velocity, Yaw Rate, and Roll Angle. The upper and lower x-axis limits were chosen based on the results of Chapter 5 .

$\mathrm{h}$ : CG height with respect to the sprung mass origin (m)

Hardware-in-the-Loop (HIL) simulation : An HIL, or Hardware-in-the-Loop, simulator is a laboratory test environment which combines computer simulation with embedded hardware. See Chapter 6.

HCU : hydraulic control unit. An integral component to any EBS system. This hydraulic block contains the pump motor, internal valves for sending brake fluid to each individual brake caliper or drum, a pressure transducer, and valve coils that are activated via the brake system ECU. Brake fluid is supplied by the brake master cylinder.

$I_{x}:$ roll moment of inertia $\left(\mathrm{kg}-\mathrm{m}^{2}\right)$

$I_{z}$ : yaw moment of inertia $\left(\mathrm{kg}-\mathrm{m}^{2}\right)$

IC : initial conditions

Inertial Navigation System (INS) : An inertial navigation system is a navigational aid that that utilizes dead-reckoning techniques to calculate the position, velocity, and orientation of a moving object which in this case is a ground vehicle. Onboard sensors include accelerometers. See Chapter 3.

$K_{\phi}:$ total roll stiffness $(\mathrm{Nm} / \mathrm{rad})$ 
$K_{\text {us }}:$ understeer coefficient

$\mathrm{L}=(\mathrm{a}+\mathrm{b}):$ wheelbase $(\mathrm{m})$

$\operatorname{lm}$ : lateral acceleration margin

max_values : This takes the maximum calculated values of lateral acceleration (Lat_Acc), lateral velocity $(v)$, yaw rate $(r)$, and roll angle $(\phi)$ and runs them through the fuzzifier.

$\mathrm{pm}$ : roll angle $(\phi)$ margin

Predictive Dynamics Model : The Matlab simulation program which contains the 3DOF equation derived in Chapter 4. The ode45 command in MATLAB ${ }^{\circledR}$ is used to integrate the 3DOF equation to solve for the Lateral Velocity, Yaw Rate, Roll Angle, and Roll Angle Rate based on vehicle and other input parameters, i.e. GPS road data. Therefore this block serves as an initial guess of the vehicle and roadway conditions. Lateral Acceleration, although not included in the final equation shown at the end of Chapter 4, is also calculated here.

Pseudorange - GPS receivers typically use an inexpensive crystal clock which is set approximately to GPS time. Thus, the clock of the ground receiver is offset from true GPS time, and because of this offset, the distance measured to the satellite differs from the "true" range. These distances are called pseudoranges since they are the true range plus a range correction resulting from the receiver clock error [2, 4-5].

$\mathrm{R}$ : radius of curvature $(\mathrm{m})$

Refresh Rate : In the simplified controller presented here several variables such as longitudinal velocity and radius of curvature were assumed to be constant. However if the driver changes his throttle position and/or adjusts the steering wheel angle the radius of curvature will change, so it is necessary for the controller to be continuously rechecking the input signals for changes. It is also necessary for the GPS to have a refresh rate so it can keep track of global changes such as abrupt changes in the actual radius of curvature of the roadway. A value of $1 \mathrm{sec}$. is common [23]. See Figures 5.1 and 5.2 .

RSC : Roll Stability Control. Roll Stability Control is an extension of the ESC systems. Just as ESC systems are designed to be yaw control devices, RSC systems are designed to be roll control devices. Using the same set of EBS sensors, code, and hardware systems will brake outside front wheels for extended period of times to sufficiently reduce the available lateral force, thus reducing and eliminating the rollover moment of the vehicle. Major suppliers are Continental Corporation, Bosch, and TRW Automotive.

$\mathrm{rm}$ : yaw rate margin

$r$ : yaw velocity (i.e., yaw rate) $(\mathrm{rad} / \mathrm{s})$

$\dot{r}$ : yaw acceleration $\left(\mathrm{rad} / \mathrm{s}^{2}\right)$ 
SWA : steering wheel angle

TCS : Traction Control. This system is like ABS but for tractive forces. There are three main types of traction control: Engine-only, Brake-only, and Engine and Brake systems. Engine-only traction control systems attempt to match a wheel torque and wheel speed model by reducing wheel slip during on-throttle applications to keep the wheels at or near their peak friction values. This is accomplished by reducing engine torque. Brake-only traction control systems apply the brakes to the driven wheels during on-throttle applications. Engine-only systems tend to be very smooth but can suffer from the time-lag associated with the reduction in engine torque. Brakeonly systems can be quicker since they reduce the wheel speeds almost instantly, but the effectiveness of these systems relies on properly bedded-in brake pads/drum shoes. Performance is reduced as pads wear and heat up. Temperature models are used to prevent pad fade, especially after multiple vehicle launches in which TCS is active. Most effective systems are ones which combine engine and brake-based systems.

TSM - Total Safety Margin - This calculation takes the calculated margins and multiplies each by a weighting factor, adds each of these up, and divides the total by the sum of the weighting factors. This new value is the Total Safety Margin and in future work would be used by the Decision Maker block to determine the overall vehicle safety condition. See Chapter 5.

u : longitudinal velocity $(\mathrm{m} / \mathrm{s})$

$v$ : lateral velocity $(\mathrm{m} / \mathrm{s})$

$\dot{v}$ : lateral acceleration $\left(\mathrm{m} / \mathrm{s}^{2}\right)$

vm : lateral velocity margin

$W_{f}$ : total front axle normal load (N)

$W_{i}$ : weighting factors for the longitudinal velocity, yaw rate, and roll angle

$W_{r}$ : total rear axle normal load (N)

$\alpha_{f, r}$ : tire slip angle (rad)

$\delta_{f}$ : front steer angle (rad)

$\phi$ : roll angle (rad)

$\dot{\phi}$ : roll velocity (rad/s)

$\ddot{\phi}$ : roll acceleration $\left(\mathrm{rad} / \mathrm{s}^{2}\right)$ 


\section{Subscripts}

$F$, f: front

$i: \mathrm{lm}, \mathrm{vm}, \mathrm{rm}, \mathrm{pm}$

inst: instantaneous, as in the instantaneous time. See Figures 5.1 and 5.2.

$R, r$ : rear 


\section{APPENDIX B}

\section{Vehicle Parameters}

\begin{tabular}{|c|c|c|c|}
\hline \multicolumn{4}{|c|}{ Truck Parameters from TruckSim 7} \\
\hline \multicolumn{4}{|c|}{ Vehicle is baseline 2 axle van, 4 rear tires, unladen } \\
\hline Parameter & Value & Unit & Description \\
\hline$G R=$ & 25 & - & Nominal Steering Gear Ratio \\
\hline$a=$ & 1.113 & $\mathrm{~m}$ & $\begin{array}{l}\text { Longitudinal distance from CG to front } \\
\text { axle }\end{array}$ \\
\hline $\mathrm{b}=$ & 3.887 & $\mathrm{~m}$ & Longitudinal distance from CG to rear axle \\
\hline $1=$ & 5 & $\mathrm{~m}$ & Wheelbase \\
\hline$h=$ & 1.173 & $\mathrm{~m}$ & Sprung Mass CG height \\
\hline$T_{f}=$ & 2.022 & $\mathrm{~m}$ & Track Width, Front \\
\hline $\mathrm{T}_{\mathrm{r}}=$ & 1.829 & $\mathrm{~m}$ & Track Width, Rear \\
\hline $\mathrm{I}_{\mathrm{x}}=$ & 8419.5 & $\mathrm{~kg}-\mathrm{m}^{2}$ & Roll Moment of Inertia \\
\hline $\mathrm{I}_{\mathrm{z}}=$ & 40344 & $\mathrm{~kg}-\mathrm{m}^{2}$ & Yaw Moment of Inertia \\
\hline$m_{s}=$ & 4457 & $\mathrm{~kg}$ & Sprung Mass \\
\hline$m_{\text {us }, \mathrm{f}}=$ & 527 & $\mathrm{~kg}$ & Unsprung Mass, Front \\
\hline$m_{\mathrm{us}, \mathrm{r}}=$ & 1004 & $\mathrm{~kg}$ & Unsprung Mass, Rear \\
\hline$m=$ & 5988 & $\mathrm{~kg}$ & Total Vehicle Mass \\
\hline$C_{\alpha f}=$ & 198043 & $\mathrm{~N} / \mathrm{rad}$ & Front Tire Cornering Stiffness, per tire* \\
\hline$C_{\alpha f, \text { total }}=$ & 396086 & $\mathrm{~N} / \mathrm{rad}$ & Total Front Tire Cornering Stiffness* \\
\hline$C_{\alpha r}=$ & 36684 & $\mathrm{~N} / \mathrm{rad}$ & Rear Tire Cornering Stiffness, per tire* \\
\hline $\mathrm{C}_{\alpha \mathrm{r}, \text { total }}=$ & 146736 & $\mathrm{~N} / \mathrm{rad}$ & Total Rear Tire Cornering Stiffness* \\
\hline$K_{\phi}=$ & 773530 & $\mathrm{Nm} / \mathrm{rad}$ & Total Roll Stiffness* \\
\hline$C_{\phi}=$ & 80842 & $\mathrm{Nms} / \mathrm{rad}$ & Total Roll Damping* \\
\hline
\end{tabular}




\section{Appendix C}

\section{Fuzzy Logic: A Technical Overview}

Fuzzy logic is a way of representing real world models in a format that is linguistic in nature. A fuzzy system can help model or control a system when we do not have a math model of how the system's output depends on its input. The fuzzy system uses commonsense rules in place of the math model, building a bridge from the input space to the output space. In the case of vehicle control, if a driver is traveling down a road and then realizes he is going too fast for the upcoming turn he would presumably react to slow the vehicle down, rather than considering the positives and negatives of slowing down, or thinking of all of the possible outcomes of what might happen if he doesn't slow down, and then make a decision based on that thought process. The goal of this chapter is to present the background of and an introduction to fuzzy logic and then discuss the process used to develop our fuzzy-based stability index.

\section{C.1 Introduction: What is Fuzzy Logic?}

Vague or fuzzy logic has a long history in mathematics and philosophy [20]. It begins with the insight that not all statements are true or false to the same degree. Some claims are truer than others and so truth is a matter of degree. This means that the old laws or axioms of either-or logic do not apply.

Fuzzy logic extends these vague or continuous logics to reasoning with vague concepts or sets. This requires a new set of algebra for the vague concepts. The fuzzy set algebra allows words to map to fuzzy sets and allows sentences to map to fuzzy rules or associations among the fuzzy sets. The rules combine to form systems or maps from an input domain to an output range.

The key idea of fuzziness comes from the multivalued logic of the 1920s: Everything is a matter of degree. A statement of fact like "The sky is blue" or "The ocean is deep" does not have a binary truth value; it has a vague or "fuzzy" truth value between 0 and 1 , and so does its negation "The sky is not blue". So the sky is both blue and not blue to some degree. This simple point of fact violates the either-or laws of logic that extend from the first formal logic of ancient Greece to the foundations of modern math and science [20].

Fuzzy logic builds gray truth into complex schemes of formal reasoning; in other words, it tries to make computers reason with our gray common sense. The earlier uses of the term fuzzy logic were the same as continuous truth or vagueness. It meant matters of degree and gray borders and thus breaking the either-or law of binary logic. Today fuzzy logic refers to a fuzzy system or mapping from input to output that depends on fuzzy if-then rules that have the form "If $X$ is $A$ then $Y$ is $B$ " where $A$ and $B$ are fuzzy sets. These fuzzy systems convert stimuli to responses or sensor measurements to control actions. Verbally the rules might have the form "If the wash water is very dirty then add much more detergent" or "If the air is cool then set the fan speed to slow”. The rules in turn depend on fuzzy sets or vague concepts like cool air, blue sky or small 
angle and these terms depend on fuzzy degrees of truth or set membership. Fuzzy logic means reasoning with vague concepts. In practice it can mean computing with words [20].

\section{C.2 History of Fuzzy Logic: Evolution of a Concept}

Fuzziness began as vagueness in the late nineteenth century [20]. Pragmatist philosopher Charles Sanders Peirce seems the first logician to have dealt with vagueness: "Vagueness is no more to be done away with in the world of logic than friction in mechanics." A concept is vague when it has blurred boundaries. For example, the concept mountain is vague because we do not know where a mountain ends and a hill begins.

Logician Bertrand Russell first identified vagueness at the level of symbolic logic. Concept A is vague if and only if it breaks Aristotle's "law" of excluded middle - if and only if A or not A fails to hold. This law fails to hold just to the extent that the "contradiction" A or not A tends to hold. Statements of logic or math obey Aristotle's laws: “ $1+1=2$ " is $100 \%$ true and $0 \%$ false. “ $1+1=3$ " is $0 \%$ true and $100 \%$ false. But statements of fact are vague and have truth values between these binary extremes. Take the color of grass for instance: “Grass is green” may be true only $80 \%$ of the time and so "Grass is not green" is true $20 \%$. Russell first saw this mismatch between gray fact and binary math and then looked for it in math itself [20].

Russell found a deeper paradox in math as he worked with Alfred North Whitehead on the pioneering volumes of Principia Mathematica. He found the ancient paradox of the liar from Crete. The Cretan says that all Cretan's lie. Does he lie or tell the truth? If he lies then he tells the truth and so does not lie. If he does not lie then he tells the truth and so he lies. Both cases lead to the contradiction $A$ and not- $A$; in other words, he both lies and does not lie simultaneously [20].

Russell found the same paradox in set theory. The set of all sets is a set. So it is a member of itself. But many sets are not members of themselves. For example, the set of apples is not a member of itself since its members are apples and not sets. But what about the set of all sets that are not members of themselves. Is it a member of itself? If it is then it is not. And if it is not then it is. Here $A$ and not- $A$ holds not in the gray world of things but in the formal system of binary mathematics [20].

Russell at first put forth his "theory of types" to ban such paradoxes but the paradoxes still emerged in other forms despite the ban. There is something deeply counterintuitive about denying that the set of all sets is not itself a set as many have done by calling it a "class". Russell saw by the time of his 1923 article "Vagueness," published in the Australian Journal of Philosophy, that we might have to relax if not reject Aristotle's law of excluded middle both to deal with paradoxes and to account for the vagueness of factual statements. Therefore formal fuzzy logic begins with this 1923 article [20].

The paradoxes motivated much of the early work in vague or fuzzy logic. Polish logician Jan Lukasiewicz made the next major advance after Russell. In the 1920s Lukasiewicz worked out the first fuzzy or multivalued logic. 
In the 1937 article "Vagueness: An exercise in logical analysis" published in the journal Philosophy of Science quantum philosopher Max Black applied multivalued logic to lists or sets of objects and drew the first fuzzy set curves. These sets $A$ are such that each object $x$ obeys or belongs to $A$ and not- $A$ to some degree and so are properly vague or fuzzy. Black followed Russell's word usage and called the sets vague. Kaplan and Schott put forth the min and max operations to define a fuzzy set algebra as did other logicians in the 1950s [20].

In 1965 Lotfi Zadeh of the University California at Berkeley published the paper "Fuzzy Sets" in the journal Information and Control. This paper first used the word fuzzy to mean "vague" in the technical literature. As a result the name fuzzy has not only persisted but largely replaced the prior term vague. Zadeh's 1965 paper applied Lukasiewicz's logic to each object in a set to work out a complete fuzzy set algebra and to extend the convex separation theorem of pattern recognition. There is some controversy because Zadeh did not refer to the works of Lukasiewicz or any of the other multivalued logicians who had previously defined the vague concepts and pointwise operators ( $\min , \max$, and $1-x$ ) at the heart of fuzzy set theory. Consequently this has often led to the perception that multivalued logic begins with fuzzy sets despite over half a century of prior work. Still, Zadeh brought about the second wave of multivalued research under the banner and language of fuzzy logic [20].

\begin{tabular}{|c|c|c|c|}
\hline Late $19^{\text {th }}$ centur & 1910 - 1913 & 1923 & 1920s \\
\hline $\begin{array}{l}\text { Charles } \\
\text { Sanders } \\
\text { Peirce: First } \\
\text { to deal with } \\
\text { vagueness }\end{array}$ & $\begin{array}{l}\text { Bertrand Russell } \\
\text { and Alfred North } \\
\text { Whitehead: } \\
\text { Principia } \\
\text { Mathematica and } \\
\text { the paradox of the } \\
\text { liar from Crete }\end{array}$ & $\begin{array}{l}\text { Bertrand Russell: } \\
\text { "Vagueness," } \\
\text { Australian } \\
\text { Journal of } \\
\text { Philosophy. } \\
\text { Formal fuzzy } \\
\text { logic begins. }\end{array}$ & $\begin{array}{l}\text { Jan Lukasiewicz: } \\
\text { worked out first } \\
\text { fuzzy or } \\
\text { multivalued logic. }\end{array}$ \\
\hline
\end{tabular}

$\begin{array}{ll}1937 & 1950 s\end{array}$

Figure C.1: Timeline of the major developments in fuzzy logic 


\section{C.3 Fuzzy Sets and Systems}

A set contains objects. A set is the theoretical primitive of mathematics just as the symbol is the theoretical primitive of logic. Set $A$ contains an object $x$ to some degree. A fuzzy set $A$ contains $x$ to some degree in $[0,1][20]$.

Consider a simple air conditioner. Fuzzy logic is used to control the fan speed in the air conditioner to adjust the temperature in the room. The air temperature in the room defines a fuzzy or linguistic variable, taking on the fuzzy-set values cold, cool, just right, warm, or hot. The process of fuzzy engineering begins with the following three steps:

Step 1: The first step picks the input and output variables $X$ and $Y$. Here the input variable $X$ is air temperature. Additional variables could be humidity or light intensity. The output $Y$ is a set of numerical motor speeds.

Step 2: The second step picks fuzzy subsets of these variables. In this case the if-part sets are cold, cool, just right, warm, and hot while the then-part sets are stop, slow, medium, fast, and blast motor speeds.

Step 3: The third step relates the output sets to the input sets in fuzzy rules, using the if-then structure described in [20]. There are several possible methods for choosing the rules: if unsure of where to begin we could make an educated guess based either on intuition or some experience; consult an expert on the system to be controlled; or use an adaptive algorithm and let the results of the current rule choices feedback to update the fuzzy rules. For this example the fuzzy rules are:

Rule 1: If the air is cold then set the motor speed to stop.

Rule 2: If the air is cool then set the motor speed to slow.

Rule 3: If the air is just right then set the motor speed to medium.

Rule 4: If the air is warm then set the motor speed to fast.

Rule 5: If the air is hot then set the motor speed to blast.

The fuzzy system $F$ maps an input $x$ to an output $F(x)$ in three steps:

Step 1: The first step matches the input $x$ to all the if-part fuzzy sets in parallel. This step "fires" or "activates" the rules by how much the input $x$ belongs to each if-part set $A$.

Step 2: The second step adds all the scaled or shrunken then-part sets into a final output set.

Step 3: The third step is "defuzzification". Many methods can be used to compute the output $F(x)$ including [16-22]:

- Center of Area

- Center of Gravity

- Center of Maxima

- Picking the mode or maximum value of the output set 
- Weighted average

An example of a practical application of fuzzy logic to vehicle control is the subway system in the Japanese city of Sendai [20]. The subway runs on a $13.6 \mathrm{~km}$ route with 16 stations. Hitachi programmed rules in a fuzzy system for acceleration and braking: If the train speed exceeds the speed threshold then reduce the speed. If the train is in the allowed zone then brake slightly (rather than accelerate). The fuzzy system gives a smoother ride than did human control and it outperforms standard PID controllers in smoothness of braking and acceleration and in electric power consumption. It also stops the subway with greater accuracy.

There are many other machines that have benefited from the application of a fuzzy logic control scheme. These include [16-22]:

Cameras and camcorders

Clothes washing machine and dryer

Microwave ovens

Mitsubishi suspension, transmission, steering, traction control, and air conditioning Nissan ABS, transmission system, fuel injection

Robot graspers

Unmanned helicopter 